(C) 2012 Zixia Huang 


\title{
SYNCHRONIZED DISTRIBUTION FRAMEWORK FOR HIGH-QUALITY MULTI-MODAL INTERACTIVE TELEIMMERSION
}

\section{BY}

\section{ZIXIA HUANG}

\section{DISSERTATION}

Submitted in partial fulfillment of the requirements for the degree of Doctor of Philosophy in Computer Science in the Graduate College of the

University of Illinois at Urbana-Champaign, 2012

\author{
Urbana, Illinois
}

Doctoral Committee:

Professor Klara Nahrstedt, Chair \& Director of Research

Professor Thomas S. Huang

Associate Professor Marco Caccamo

Dr. Thomas Y. Woo, Bell Laboratories, Alcatel-Lucent 


\section{ABSTRACT}

This dissertation investigates issues of performing synchronized distribution of time-correlated multi-modal continuous media data in the distributed interactive teleimmersion, and proposes approaches to solve these problems. The current best-effort shared Internet is unreliable in supporting bandwidth-savvy multimedia streaming, and frequent network jitter can contribute to the heterogeneity of distribution latencies (a.k.a., synchronization skews) within and across the multi-source sensory streams over time. The system limitations and computation constraints at the end devices can also prohibit the multimedia data in preserving the synchronization dependencies. Existing studies usually control synchronization skews by inserting buffering latencies at the media receivers. But due to the real-time nature of interactive multimedia in both two-site and multi-site applications, these approaches can impact the communication interactivity and media signal intelligibility, and affect the subjective satisfactions of end users.

To address these issues, we propose a novel and systematic synchronization framework for teleimmersion, and more generally, distributed interactive multimedia systems. We first discuss a new multidimensional synchronization specification model that captures the scalability of multi-modal devices, the heterogeneity of multimedia, and the diversity of the end user activities in the next-generation multimedia applications. An adaptive decision policy is also included for identifying the time correlations of multimedia frames captured from distributed media sensors. Based on the new model, we then develop collaborative multi-tier synchronization controls that consistently minimize the multimedia skews during media processing and distribution. Specifically, at the sender capturing tier, we present a dynamic computation resource provisioning algorithm to bound the skews arising during multimedia computation. At the distribution tier, we design a synchronized multi-stream distribution overlay with cooperative bandwidth management according to the diverse end user interests. At the receiver presentation tier, we perform presentation scheduling scheme to realize the multimedia synchronization at the output devices. To evaluate the tradeoff of the synchronization quality, media signal intelligibility and interactive quality, and their interactions with end user perception, we further conduct extensive subjective evaluations, and utilize the real perceptual feed- 
back to drive the media presentation adaptation. We elaborate on the implementation of the synchronization service component in our Illinois TEEVE (Teleimmersive Environment for EVErybody) testbed. Repeatable experimental results from both the teleimmersive emulator and the real TEEVE system confirm the effectiveness of our framework. 
To my parents 


\section{ACKNOWLEDGMENTS}

First and foremost, my deepest gratitude goes to my Ph.D. research advisor, Prof. Klara Nahrstedt, for giving me the opportunity of joining the TEEVE project. I appreciate all her stimulating discussions and meaningful advice throughout my Ph.D. study. I have learnt a lot from her broad knowledge, critical insights and thoughtful directions, and have benefited from her professionalism and dedication.

I would also sincerely thank my honorable Ph.D. committee members, Prof. Thomas S. Huang, Prof. Marco Caccamo and Dr. Thomas Y. Woo, for the technical discussions and constructive feedback on My Ph.D. Preliminary presentation and my final dissertation. Their comments play an essential role in finalizing this dissertation.

I would like to extend my special thanks to my previous M.Sc. advisor, Prof. Benjamin W. Wah for leading me into the multimedia networking research area.

Many thanks go to Wanmin Wu, Raoul Rivas, Ahsan Arefin, Shu Shi, Pengye Xia, Pooja Agarwal, Ying Huang, Long Vu, Hoang Nguyen, Thadpong Pongthawornkamol and all other members in the Multimedia Operating Systems and Networking (MONET) research group for their friendly assistance and beneficial group discussions.

Last, but not least, I would like to express my earnest gratitude to my father Shen Huang, and my mother Jianping Xia, who consistently give me encouragement, support, and love. This dissertation is dedicated to them.

This research was supported in part by National Science Foundation Grant CNS 0720702, 0834480, 0964081, 1012194, IIP 1110178, by UPCRC grant from Intel and Microsoft, and by the Grainger Grant. 


\section{TABLE OF CONTENTS}

LIST OF TABLES $\ldots \ldots \ldots \ldots \ldots \ldots \ldots \ldots \ldots \ldots \ldots \ldots$

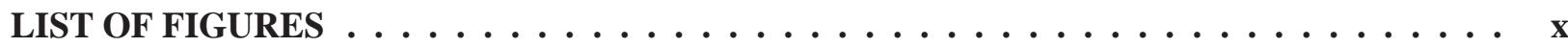

LIST OF ABBREVIATIONS ........................ . . . . . . . .

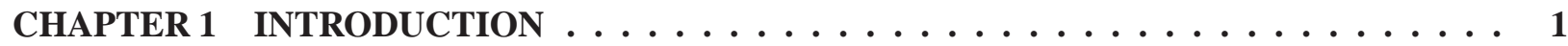

1.1 System Configurations of Teleimmersion $\ldots \ldots \ldots \ldots \ldots \ldots$

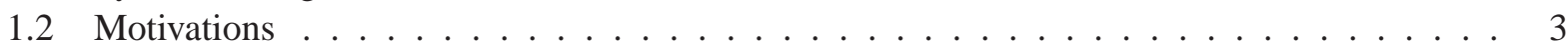

1.3 Synchronization Demands in Teleimmersion . . . . . . . . . . . . . 5

1.3.1 Types of Synchronization Demands . . . . . . . . . . . . . . . . . 5

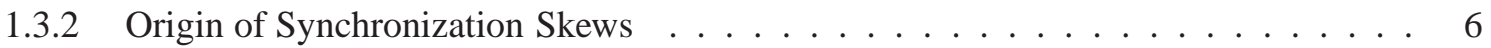

1.4 Synchronization and Human Perception . . . . . . . . . . . . . . . . . . 9

1.4.1 Impact of Synchronization Controls . . . . . . . . . . . . . . . . . . . . . 9

1.4 .2 Impact of Human Perception . . . . . . . . . . . . . . . . . . . 10

1.5 Thesis Problem Statement . . . . . . . . . . . . . . . . . . . . . . 12

1.5.1 P1: Synchronization Specification . . . . . . . . . . . . . . . 12

1.5.2 P2: Multi-tier Collaborative Synchronization Control . . . . . . . . . . . . . . . 14

1.5.3 P3: Synchronization and Human Perception . . . . . . . . . . . . . . . . . 15

1.5.4 P4: Implementation of Synchronization Service Component . . . . . . . . . . . 17

1.6 Research Approaches . . . . . . . . . . . . . . . . . . . . . . . 17

1.7 Research Contributions . . . . . . . . . . . . . . . . . . . . 18

1.8 Dissertation Outline . . . . . . . . . . . . . . . . . . . . . . . 19

1.9 Table of Abbreviations and Notations . . . . . . . . . . . . . . . . . 20

CHAPTER 2 RELATED WORK . . . . . . . . . . . . . . . . . . 21

2.1 Historical View of Synchronization Studies . . . . . . . . . . . . . . . . . . 21

2.1.1 Years of Birth: On and Before $1988 \ldots \ldots \ldots \ldots \ldots \ldots \ldots$

2.1.2 Years of Understanding: 1989-1994 . . . . . . . . . . . . . . . 23

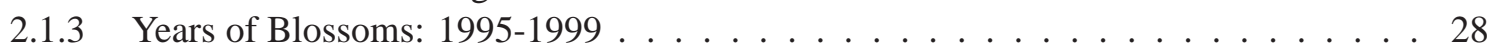

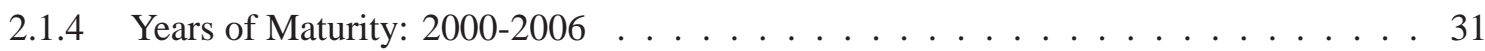

2.1.5 Years of Leaps: On and After $2007 \ldots \ldots$. . . . . . . . . . . . . . 33

2.2 Subjective Rating Method and Subjective Metrics . . . . . . . . . . . . . . . 36

2.2.1 Subjective Rating Method . . . . . . . . . . . . . . . . . . . . 36

2.2 .2 Subjective Metrics . . . . . . . . . . . . . . . . . . . . . . . . . . . . . . . .

2.3 Human Perception in Multimedia Applications . . . . . . . . . . . . . . . 42

2.3 .1 Survey Description . . . . . . . . . . . . . . . . . . . 42 
2.3 .2 Media Signal Intelligibility . . . . . . . . . . . . . . . . . . . . 44

2.3 .3 Synchronization Quality . . . . . . . . . . . . . . . 45

2.3 .4 Interactivity . . . . . . . . . . . . . . . . . . 46

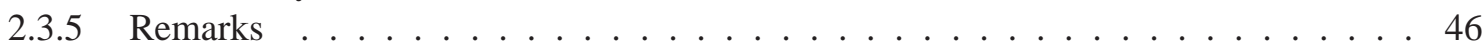

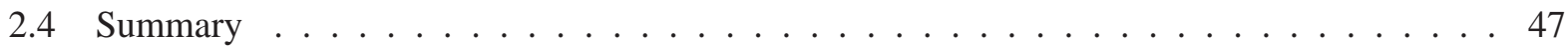

2.5 Table of Abbreviations and Notations _ . . . . . . . . . . . . . . . . 47

CHAPTER 3 MODELING OF SYNCHRONIZATION SPECIFICATION _ . . . . . . . . . 49

3.1 Mathematical Denotations . . . . . . . . . . . . . . . . . . . . . 49

3.2 A Multi-dimensional Synchronization Model . . . . . . . . . . . . . . . . . 50

3.2.1 Dimension of Synchronization Demands . . . . . . . . . . . . . . 50

3.2.2 Dimension of Synchronization Locations . . . . . . . . . . . . . . . . 51

3.2.3 Dimension of Synchronization Reference Hierarchy . . . . . . . . . . . . . . . . . 52

3.2 .4 Definition of Synchronization Skews . . . . . . . . . . . . . . . . 54

3.3 Time Correlations of Multi-modal Media Frames . . . . . . . . . . . . . . . . 55

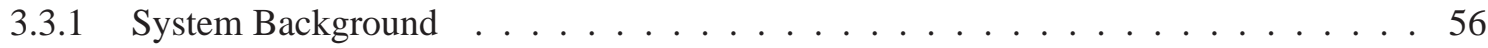

3.3 .2 Problem Formulation . . . . . . . . . . . . . . . . . . 57

3.3 .3 Design Rationales . . . . . . . . . . . . . . . . . . . . 60

3.3.4 Identification Algorithm for Intra-media SP . . . . . . . . . . . . . . . . . . 60

3.3.5 Identification Algorithm for Intra-bundle SP . . . . . . . . . . . . . . . . . . . . 63

3.3.6 Impact of Real System Challenges . . . . . . . . . . . . . . . . . 65

3.3.7 Evaluation Results . . . . . . . . . . . . . . . . . . . . . . 67

3.3 .8 Remarks . . . . . . . . . . . . . . . . . . . . . . 69

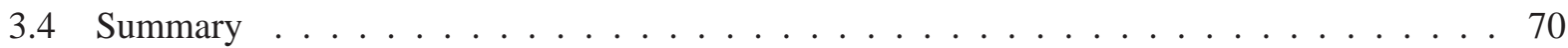

3.5 Table of Abbreviations and Notations . . . . . . . . . . . . . . 70

CHAPTER 4 DESIGN OF MULTI-TIER SYNCHRONIZATION CONTROLS $\ldots \ldots \ldots$

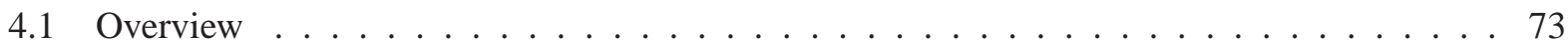

4.2 CloudStream: Capturing Tier Control . . . . . . . . . . . . . . . . . . . 74

4.2.1 Design of CloudStream . . . . . . . . . . . . . . . . . 75

4.2.2 Evaluation Results . . . . . . . . . . . . . . . . . . . . . . 77

4.3 SyncCast: Distribution Tier Control _ . . . . . . . . . . . . . . . . . . 78

4.3 .1 System Model . . . . . . . . . . . . . . . . . . . . . . 79

4.3 .2 Problem Formulation . . . . . . . . . . . . . . . . . . . . . . . . . . . . . . .

4.3.3 Path Selection Policies . . . . . . . . . . . . . . . . . . . . 82

4.3 .4 Design of SyncCast . . . . . . . . . . . . . . . . . . . . . . 84

4.3.5 Cooperative Frame Rate Allocation . . . . . . . . . . . . . . . . . . 87

4.3.6 Evaluation Results . . . . . . . . . . . . . . . . . . . . . 87

4.4 TBuffer: Presentation Tier Control . . . . . . . . . . . . . . . . . . . . . . 93

4.4 .1 Problem Formulation . . . . . . . . . . . . . . . . . . . . . . 94

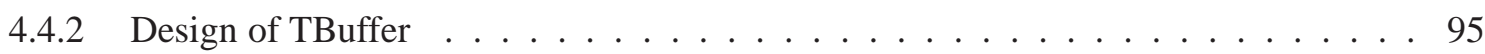

4.4 .3 Evaluation Results . . . . . . . . . . . . . . . . . . . 95

4.5 Summary . . . . . . . . . . . . . . . . . . . . . . . . . 99

4.6 Table of Abbreviations and Notations . . . . . . . . . . . . . . . . . . 99 
CHAPTER 5 SYNCHRONIZATION AND HUMAN PERCEPTION . . . . . . . . . . . . 102

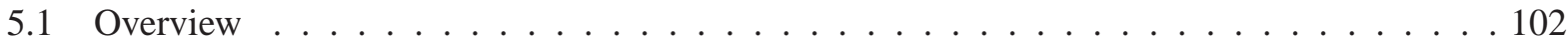

5.2 Studied Teleimmersive Shared Activities . . . . . . . . . . . . . . . . 103

5.3 Objective Quality Metrics Impacting Human Perception . . . . . . . . . . . . . . . . 103

5.3.1 Objective Metrics for Streaming Media Quality . . . . . . . . . . . . . . . . . . . . 104

5.3.2 Objective Metrics for Flicker Effect . . . . . . . . . . . . . . . . . . 106

5.4 Description of User Study ～. . . . . . . . . . . . . . . . . . 107

5.4 .1 Methodology . . . . . . . . . . . . . . . . . . . 107

5.4 .2 Preparation of Teleimmersive Media Samples . . . . . . . . . . . . . . . . . 108

5.4 .3 Setup of User Study . . . . . . . . . . . . . . . . . . . . . . . . . 109

5.4 .4 Subjective Evaluation Results . . . . . . . . . . . . . . . . . . . . . 109

5.4 .5 Remarks . . . . . . . . . . . . . . . . . . . . . 115

5.5 Perception-driven Presentation Scheduling (MPS) for Multimedia Synchronization . . . . 118

5.5.1 New Subjective Metric: Preference . . . . . . . . . . . . . . . . . . . . . . . 119

5.5.2 Interactions between MPS and Human Perception . . . . . . . . . . . . . . . . . 119

5.5 .3 Design of Perception-driven MPS . . . . . . . . . . . . . . . 121

5.5 .4 Evaluation Results . . . . . . . . . . . . . . . . . . . 125

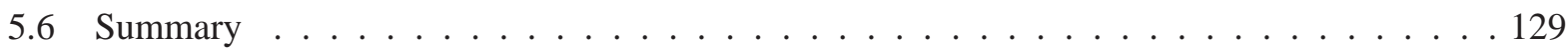

5.7 Table of Abbreviations and Notations _ . . . . . . . . . . . . . . . . 129

CHAPTER 6 SYNCHRONIZATION IMPLEMENTATION IN TELEIMMERSION . . . . . 132

6.1 An Introduction of Real Teleimmersive System Implementation ～. . . . . . . . . . . 132

6.2 Implementation of Synchronization Service Components . . . . . . . . . . . . . 133

6.2 .1 Implementation Overview . . . . . . . . . . . . . . . . . . . . 133

6.2 .2 RTP Format . . . . . . . . . . . . . . . . . . . . . . . . . . 134

6.2 .3 Clock Synchronization . . . . . . . . . . . . . . . . . . . . . 134

6.2 .4 Identification of Synchronization Points . . . . . . . . . . . . . . 136

6.2 .5 Congestion Detection and Avoidance . . . . . . . . . . . . . . . . 137

6.2.6 Intra-session Synchronization Mechanism . . . . . . . . . . . . . . . . . 138

6.2.7 Receiver Presentation Scheduling . . . . . . . . . . . . . . . . . . . 138

6.3 Performance Validation . . . . . . . . . . . . . . . . . . . . . . . . . 139

6.3 .1 Test Scenarios . . . . . . . . . . . . . . . . . . . . . . . . . . . . . . . . 139

6.3 .2 Evaluation Results . . . . . . . . . . . . . . . . . . . . 139

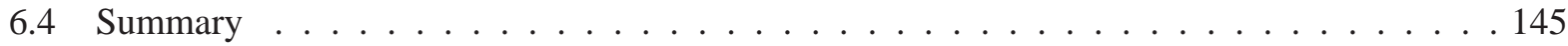

6.5 Table of Abbreviations and Notations _ . . . . . . . . . . . . . . . 145

CHAPTER 7 CONCLUSIONS AND FUTURE WORK $\ldots \ldots \ldots \ldots \ldots \ldots \ldots$

7.1 Conclusions . . . . . . . . . . . . . . . . . . . . . . . 147

7.2 Lessons Learned . . . . . . . . . . . . . . . . . . . . . . . . . 148

7.3 Future Work . . . . . . . . . . . . . . . . . . . . . . . . . . 149

7.3.1 Standardizing Synchronization Protocols . . . . . . . . . . . . . . . . . . . 149

7.3.2 Mobile Synchronization . . . . . . . . . . . . . . . . . . 150

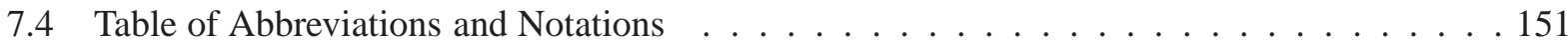

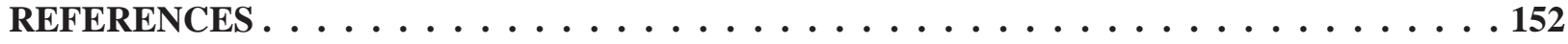




\section{LIST OF TABLES}

2.1 Absolute Category Rating. . . . . . . . . . . . . . . . . . . . . . 36

2.2 Degradation Category Rating. . . . . . . . . . . . . . . . . . . . . 37

2.3 Comparative Category Rating. . . . . . . . . . . . . . . . . . . . . 38

2.4 An example of user votes. . . . . . . . . . . . . . . . . . . . . . 38

2.5 A survey of subjective quality assessment. The types of studied multimedia systems include audio and 2D video conferencing (CONF), 2D video-on-demand (VOD), 3D stereoscopic video-on-demand (STEREO) and teleimmersive (TI) applications. VSI: video signal intelligibility, which includes the video frame rate (FR), the spatial resolution (RES), the encoding quality (ENC) and the 3D-specific factors (3D). ASI: audio signal intelligibility. Three subjective rating methods are classified: ACR, CCR and DCR. Y: representing the corresponding quality dimension is studied. . . . . . . . . . 43

4.1 Average one-way delay (msec) for 5 nodes. (a) in US, (b) in US and Europe, (c) in US, Europe and Asia. . . . . . . . . . . . . . . . . . . . . . . . 88

4.2 One-way delay (msec) for 9 nodes. (a) in US, (b) in US and Europe, (c) in US, Europe and China. . . . . . . . . . . . . . . . . . . . . . . . . . . . 89

5.1 Discretization of quality metrics in $\vec{e}$. HRD $=800 \mathrm{~ms}$ is used in computing $e_{D}$ in CONV (Section 3.2). $e_{V}$ is rounded to the nearest integer in the evaluation. . . . . . . . 108

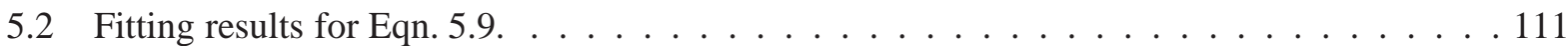

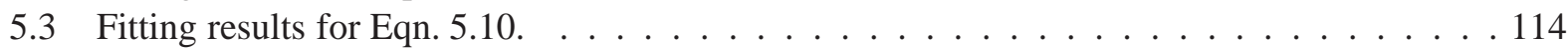

5.4 Comparisons for CONV and COLL characteristics. Note that H/L mean comparatively more/less important between the two application. . . . . . . . . . . . . 118

6.1 Expanded RTP header in teleimmersion . . . . . . . . . . . . . . . 135

6.2 Case 1: $D_{r g}$ and $D_{r l}$ of two video and one audio streams, as well as the resulting skews. Media data are sent from site $n^{x_{0}}$ to site $n^{y_{0}} . \Delta D_{r l}\left(m_{A}^{x_{0}}, n^{y_{0}}\right)$ : intra-bundle (audiovisual) skew, $\Delta D_{r l}\left(s_{V, 2}^{x_{0}}, n^{y_{0}}\right)$ : intra-media (video) skew. Unit: ms. . . . . . . . . . . 142

6.3 Case 2: $D_{r g}$ and $D_{r l}$ of one video stream and one audio stream from a single sender site $n^{x_{0}}$ to two receiver sites $n^{y_{0}}$ and $n^{y_{1}}$, as well as the resulting skews. $\Delta D_{r l}\left(m_{A}^{x_{0}}, n^{y_{0}}\right)$, $\Delta D_{r l}\left(m_{A}^{x_{0}}, n^{y_{1}}\right)$ : intra-bundle (audio-visual) skew. $n^{y_{1}}$ is the reference site, and $\Delta D_{r l}\left(u^{x_{0}}, n^{y_{0}}\right)$ is the intra-session skew. Unit: ms. . . . . . . . . . . . . . . . . . . . . . 142

6.4 Case 3: $D_{r g}$ and $D_{r l}$ of one video stream and one audio stream from two sender sites $n^{x_{0}}$ and $n^{x_{1}}$ to a single receiver site $n^{y_{0}}$, as well as the resulting skews. $\Delta D_{r l}\left(m_{A}^{x_{0}}, n^{y_{0}}\right)$, $\Delta D_{r l}\left(m_{A}^{x_{1}}, n^{y_{0}}\right)$ : intra-bundle (audio-visual) skew. $n^{x_{1}}$ is the reference site, and $\Delta D_{r l}\left(u^{x_{0}}, n^{y_{0}}\right)$ is the intra-session skew. Unit: ms. . . . . . . . . . . . . . . . . . . . . 142 


\section{LIST OF FIGURES}

1.1 Illinois TEEVE: a teleimmersive environment. . . . . . . . . . . . . . . . . 2

1.2 Multi-modal multi-stream configuration in multi-site teleimmersive systems. . . . . . . . 3

1.3 Four types of synchronization demands. $s_{i, j}$ denotes each sensory stream, where $j$ is the stream index, and $i=$ ' $\mathrm{V}$ ', 'A' and ' $\mathrm{H}$ ' represent the video, audio, haptic streams, respectively. 6

1.4 Computation time for two video streams $s_{\mathrm{V}, 1}$ and $s_{\mathrm{V}, 2}$, and one audio stream $s_{\mathrm{A}, 1} \ldots \ldots$

1.5 Overlay topology and issue of intra-media and intra-session synchronization. The sites are represented by circles and the paths are indicated in curves. $s_{V, j}^{x}$ denotes the video stream from sender site $x$, where $j$ is the stream index, and $u^{x}$ represents the media bundle from sender site $x$. The numbers by the paths represent the Internet average latency $($ unit: $\mathrm{ms}) \ldots \ldots \ldots \ldots \ldots \ldots$

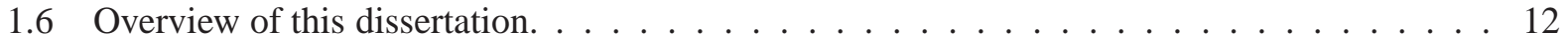

1.7 An example of time-correlated media frames for one video and two audio streams. . . . . 13

2.1 (a) NTP clock offset computation, (b) NTP multi-stratum hierarchy. . . . . . . . . . . 23

2.2 Three synchronization classification models. . . . . . . . . . . . . . . . . . . 24

2.3 Three synchronization specification models. . . . . . . . . . . . . . 25

2.4 Group synchronization control: sender-based and receiver-based synchronization. S: sender site, R: receiver site. . . . . . . . . . . . . . . . . . . . . . . . . . 29

2.5 Multi-device synchronization. Option 1: synchronization module is one of the device machine; Option 2: synchronization model is the specialized machine (i.e., receiver gateway). 35

3.1 Our proposed multi-dimensional synchronization model. . . . . . . . . . . . . 50

3.2 Illustration of media sensory devices and computation machines at the sender sites. . . . . 55

3.3 (a) Video inter-frame period; (b) Difference of expected and actual captured timestamp for a USB microphone with clock drift, measured at the USB sound card. . . . . . . . . . . 56

3.4 An example of intra-media SP sequence for three body sensory streams. Each solid block indicates a media frame. The solid circular rectangles represent the intra-media SP sequence, whose assigned captured timestamp $t_{c}$ has a periodicity of $\Delta T_{B} \ldots \ldots$. . . 58

3.5 An example of intra-bundle SP sequence. Each dotted block indicates an intra-media SP. The dotted surrounding curves represent the intra-bundle SP sequence, whose assigned captured timestamp $t_{c}$ has a periodicity of $\Delta T_{u} \ldots \ldots \ldots \ldots \ldots \ldots$

3.6 Two options of intra-media SP/SR (between solid lines) and resulting best assigned captured timestamp $t_{c}^{*}$ (dotted lines) for body sensory streams. Each block indicates a media

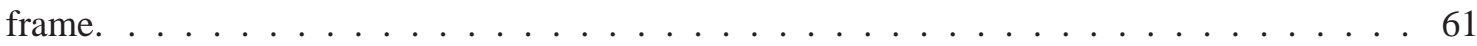

3.7 Two options of intra-bundle SP. Each dotted block indicates an intra-media SP. . . . . . . . 63 
3.8 Distribution of $t_{c}-t_{e}$ for video and audio frames in the real teleimmersive testbed. (a) A snapshot of 50 video frames; (b) Distribution of all video frames; (c) A snapshot of 400 audio frames; (d) Distribution of all audio frames.

3.9 Fig.(a-b) show video intra-media SP identification with no drift. X-axis: $\sigma_{V}$. (a) Probability of a correct SP $(\hat{k}=k)$, (b) Probability of a wrong SP $(\hat{k}=k+1)$. The 3 curves in each figure: streams with different $\beta_{V, j}$ (i.e., $\beta_{V, j}=0,5,10 \mathrm{~ms}$ ). Fig.(c-d) show the impact of audio drift $\gamma_{A}>1$ on intra-media SP identification for stream $s_{A, 1}$ with $\beta_{A, 1}=0$. (c) Probability of a correct SP $(\hat{k}=k)$, (d) Probability of a wrong SP $(\hat{k}=k+1)$. The 4 curves in each figure: different $\left(\sigma_{A}, \gamma_{A}\right)$ pairs. . . . . . . . . . 69

4.1 Multi-tier synchronization control timeline. . . . . . . . . . . . . . . . 74

4.2 An illustration of CloudStream setup. . . . . . . . . . . . . . . . . . 75

4.3 Multi-level parallelization in the cloud. DP: data partition within 3D singleview video frame. $N S$ : number of views (video streams), $M$ : number of DP within each stream. . . . 76

4.4 Average computation time of video frames using single or dual CPU for under three scenarios: no person in the scene, side view, and front view. . . . . . . . . . . . . . . 77

4.5 Left figure shows the computation time for each video frame using single CPU. Right figure depicts the minimal number of CPU required to achieve synchronization con-

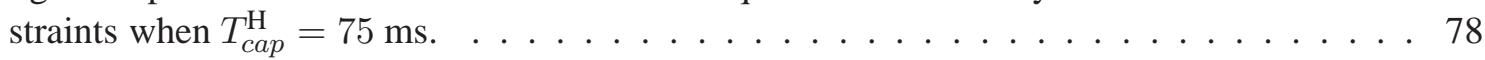

4.6 (a) An example of different path options (numbers are indicated in ms). (b) An example of video dominance and path prioritization . . . . . . . . . . . . . . . 79

4.7 An overview of SyncCast. . . . . . . . . . . . . . . . . . . . . . 84

4.8 Number of victim sites in ViewCast. $\mathrm{X}$-axis represents $\delta_{3}$. Number of victim sites in SyncCast is always zero. . . . . . . . . . . . . . . . . . . . 90

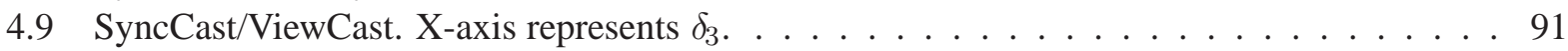

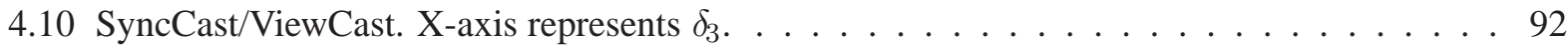

4.11 Timeline at the receiver gateway . . . . . . . . . . . . . . . 93

4.12 $D_{r g}$ of each sensory stream evaluated at intra-bundle synchronization point, and the resulting $D_{r l}$ adaptation. Alg 1: our TBuffer algorithm; Alg 2: traditional single-reference algorithm. Note that in (a),(d), the two $D_{r l}$ curves of Alg 1 and 2 overlap. Superscript indicating the sender site is omitted in all denotations in the figure. . . . . . . . . . . 97

$4.13 s_{V, 3}^{1}$ is the video reference stream. (a) Snapshot of $\Delta D_{r g}\left(s_{V, 1}^{1}\right)$; (b) Percentile of synchronization skew: $\Delta D_{r g}\left(s_{V, 1}^{1}\right), \Delta D_{r g}\left(s_{V, 2}^{1}\right)$ and $\Delta D_{r g}\left(m_{V}^{1}\right)$; (c-d) Resulting synchronization skew due to receiver adaptation: $\Delta D_{r l}\left(s_{V, 1}^{1}\right), \Delta D_{r l}\left(s_{V, 2}^{1}\right)$ and $\Delta D_{r l}\left(m_{V}^{1}\right)$. Alg 1: our TBuffer algorithm; Alg 2: traditional single-reference algorithm. In (c), $\Delta D_{r l}\left(s_{V, 1}^{1}\right)=\Delta D_{r l}\left(s_{V, 2}^{1}\right)=0$. Superscript indicating the sender site is omitted in all denotations in the figure. . . . . . . . . . . . . . . . . . . 98

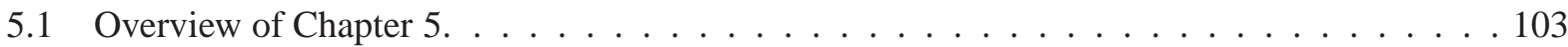

5.2 Two Teleimmersive applications evaluated in our user study. . . . . . . . . . . . . . 104

5.3 Interactivity in conversation-oriented activity. . . . . . . . . . . . . . . 106

5.4 Interactivity in collaborative activity $(\mathrm{ACT}) \ldots \ldots \ldots \ldots$

5.5 CMOS and 95\% confidence intervals comparing optimal reference $\vec{e}^{*}$ (first sample in the comparison) and $e_{V}$-degraded samples (but optimal $\left.e_{A}, e_{D}, e_{S}\right) \ldots \ldots \ldots$ 
5.6 CMOS results and 95\% confidence intervals. (1) and (3) show the comparisons between the optimal reference $\vec{e}^{*}$ (first sample in the comparison) and a sample with a degraded $e_{S}$ (but with the same optimal $e_{V}, e_{A}, e_{D}$ ). (2) and (4) show the two samples with different $e_{S}$, but same $e_{V}, e_{A}$, and $e_{D}=800 \mathrm{~ms}$ for CONV and $0 \mathrm{~ms}$ for COLL. The first sample in the comparison is $e_{S}=0$ and the second is $e_{S}= \pm 150 \mathrm{~ms} \ldots \ldots \ldots 11$

5.7 CMOS results and 95\% confidence intervals. (1) and (3) show the comparisons between the optimal reference $\vec{e}^{*}$ (first sample in the comparison) and a sample with a degraded $e_{D}$ (but with the same optimal $e_{V}, e_{A}$ and $e_{S}$ ). G.107 and G.1070 delay curves are also drawn in (1). (2) and (4) show the results for two samples with different $e_{D}$, but same $e_{V}, e_{A}$, and $e_{S}=0$. The first sample in the comparison is $e_{D}=800 \mathrm{~ms}$ for CONV and $0 \mathrm{~ms}$ for COLL, and the second is with the degraded $e_{D}$ as indicated in the figures. . . . . 113

5.8 Comparison results $\left(N_{>0}, N_{=0}, N_{<0}\right)$ for samples in CONV. All samples with $e_{A}=$ 4.0 and $e_{S}=0$. The caption format is $\left(e_{V}^{1}, e_{D}^{1}\right)$ vs $\left(e_{V}^{2}, e_{D}^{2}\right)$, where the four numbers are

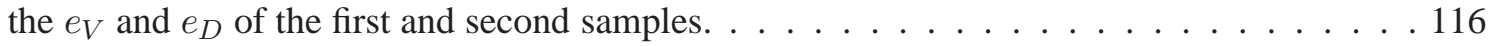

5.9 Comparison results $\left(N_{>0}, N_{=0}, N_{<0}\right)$ for samples in COLL. All samples with $e_{A}=4.0$ and $e_{S}=0$. The caption format is $\left(e_{V}^{1}, e_{D}^{1}\right)$ vs $\left(e_{V}^{2}, e_{D}^{2}\right)$, where the four numbers are the $e_{V}$ and $e_{D}$ of the first and second samples. . . . . . . . . . . . . . 117

5.10 Tradeoffs among objective metrics of the teleimmersive streaming media quality. . . . . 120

5.11 Interaction of quality metrics with control variables . . . . . . . . . . . . 121

5.12 Grey dots: all non-dominated points on an operating plane. Red boxes: local perceptual optima returned by our perception-based genetic algorithm. . . . . . . . . . . . 125

5.13 Comparison between perception-driven MPS (A1) and non-perception based algorithm (A2) [1]. Internet traces are between IL,USA to the Netherlands. Bandwidth is $15 \mathrm{Mbps}$. $D_{V}$ and $D_{A}$ are defined in Eqn. 5.12 and 5.13. Flicker values are shown for each receiver

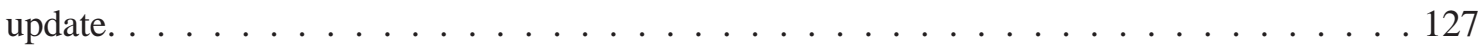

5.14 Objective quality metric values for each 5-sec duration. A1: perception-driven MPS. A2: non-perception based algorithm. . . . . . . . . . . . . . 128

6.1 Configuration and implementation of real teleimmersive systems. . . . . . . . . . 133

6.2 Implementation overview of synchronization service components in teleimmersion. . . . . 134

6.3 Hierarchical virtual clock synchronization in teleimmersion. . . . . . . . . . 136

6.4 Identifying the synchronization points. . . . . . . . . . . . . . 137

6.5 Intra-session synchronization in teleimmersion. Unit: ms. . . . . . . . . . . . . . . 138

6.6 Three cases for synchronization evaluation in real teleimmersion. . . . . . . . . . . . 140

6.7 Case 1: two-party synchronization evaluation. Audio/video traffics with an average delay of $85 \mathrm{~ms}$ and jitter of $40 \mathrm{~ms}$. . . . . . . . . . . . . . . . . . . . . . 141

6.8 Case 2: inter-receiver synchronization evaluation. Receiver 1: audio/video traffics with no delay and jitter. Receiver 2: audio/video traffics with an average delay of $100 \mathrm{~ms}$ and jitter of $15 \mathrm{~ms} . \ldots \ldots \ldots \ldots \ldots$. . . . . . . . . . . . . . . . . . . . . . . . . . . . . . . .

6.9 Case 3: inter-sender synchronization evaluation. Sender 1: audio/video traffics with no delay and jitter. Sender 2: audio/video traffics with an average delay of $150 \mathrm{~ms}$ and jitter of $40 \mathrm{~ms}$. 


\section{LIST OF ABBREVIATIONS}

\begin{tabular}{ll} 
TEEVE & Teleimmersive Environment for EVErybody \\
ITU & International Telecommunication Union \\
IETF & Internet Engineering Task Force \\
NTP & Network Time Protocol \\
PTP & Precision Time Protocol \\
MOS & Mean-Opinion-Score \\
DMOS & Degradation MOS \\
CMOS & Comparative MOS \\
ACR & Absolute Category Rating \\
CCR & Comparative Category Rating \\
DCR & Degradation Category Rating \\
PESQ & Perceptual Evaluation of Speech Quality \\
QoS & Quality of Service \\
QoE & Quality of Experience \\
CI & Confidence Interval \\
VOD & Video-On-Demand \\
CIF & Common Intermediate Format \\
QCIF & Quarter Common Intermediate Format \\
SVM & Support Vector Machine \\
SP & Synchronization Point \\
WR & Synchronization Region \\
W.L.G & Without Loss of Generality \\
\hline
\end{tabular}




$\begin{array}{ll}\text { MNOG } & \text { Massively Multiplayer Online Game } \\ \text { CSCW } & \text { Computer Supported Cooperative Work } \\ \text { TCP } & \text { Transmission Control Protocol } \\ \text { UDP } & \text { User Datagram Protocol } \\ \text { IP } & \text { Internet Protocol } \\ \text { RTP } & \text { Real-time Transport Protocol } \\ \text { RTCP } & \text { Real-time Transport Control Protocol } \\ \text { HTTP } & \text { Hypertext Transfer Protocol } \\ \text { IPTV } & \text { Internet Protocol based TV } \\ \text { VoIP } & \text { Voice over Inter Protocol } \\ \text { EED } & \text { End-to-End Delay } \\ \text { HRD } & \text { Human Response Delay } \\ \text { CONV } & \text { Conversational Activity } \\ \text { COLL } & \text { Collaboration Activity } \\ \text { LAN } & \text { Local Area Network } \\ \text { MPS } & \text { Media Presentation Scheduling }\end{array}$




\section{CHAPTER 1}

\section{INTRODUCTION}

\subsection{System Configurations of Teleimmersion}

Distributed interactive teleimmersive applications can offer a joint holographic environment where distributed users at different geographical locations are able to conduct shared activities with an unmatched realistic experience. Unlike the commercial teleconferencing or telepresence systems $[2,3,4]$, where users may find themselves talking to the screen, teleimmersive applications can enhance the traditional remote communication style by allowing full-body interactions in an immersive collaboration. Apart from the conferencing capability, useful applications have also been found in the medical consultation, remote education, cyberarcheology and collaborative gaming [5,6]. Fig. 1.1 demonstrates our Illinois teleimmersive testbed named TEEVE, i.e., Teleimmersive Environment for EVErybody.

When building the next-generation teleimmersive applications, three characteristics have become essential for achieving a realistic space.

- Multiple media modalities. A teleimmersive site usually features a variety of multimedia sensors with heterogeneous media modalities in order to capture human interactions in different aspects. For example, in our TEEVE testbed, we have 3D multiview/stereoscopic cameras, microphones, accelerometers, haptic and body sensors, which produce 3D video, spatial audio, accelerometer, haptic and body sensory streams in real time. This will allow our users to enjoy a vivid and all-around experience during the teleimmersive session. In multimedia studies, the term "session" indicates a status of the interactive media communications between two or more sites for a shared collaboration.

- Multiple sensory devices of same media modality. To preserve the spatiality and directionality of physical room environment, each site is configured with multiple sensory devices of the same media modality to capture the a physical person or object from different angles. For example, we have setup up to 8 multiview cameras spacing 45 degrees in angle in each TEEVE testbed. 


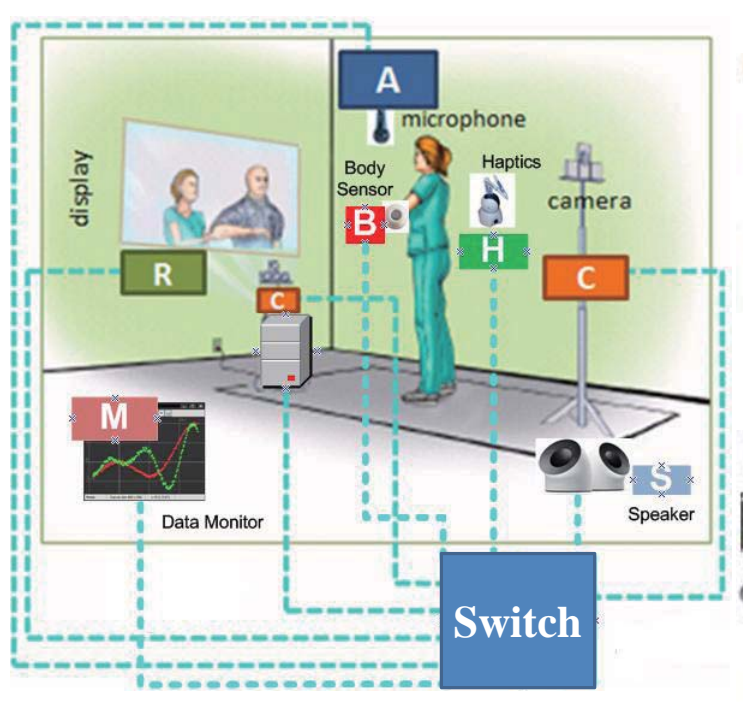

Media Sensory Inputs:

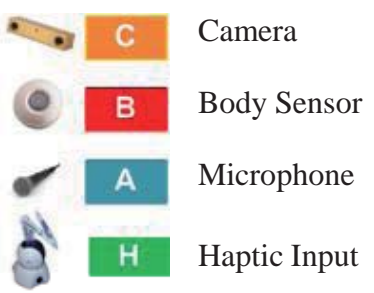

Media Sensory Outputs:

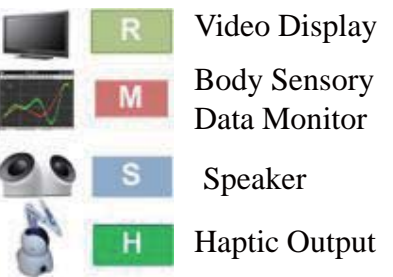

Figure 1.1: Illinois TEEVE: a teleimmersive environment.

- Multiple sender and receiver sites. Multiple users can join the same teleimmersive activity at the same time, so there can be multiple sender and receiver sites in a single session. There are two types of receiver users. One is the active receiver, which itself is also a sender site capturing and disseminating multimedia streams. The other is called the passive receiver, which is only responsible for decoding and presentation of received multimedia data. Unless otherwise specified, we only study the category of active receivers in this dissertation. Note that, depending on the selected view angles of the media presentation, multiple receivers may exhibit heterogeneous interests in different portions of sensory streams from the same sender site.

To support the multi-modal multi-stream system configuration in the multi-site teleimmersion, the overall system architecture can be divided into three tiers, as shown in Fig. 1.2.

- Capturing tier. Each sender site is configured with a set of multi-modal sensory devices (e.g., multiple 3D cameras or body sensors) to capture time-correlated multimedia multi-streams. A specialized machine is usually attached to each sensory device for media processing and encoding.

- Distribution tier. Each sender gateway aggregates the encoded multimedia streams and sends the streaming bundle to the remote receiver sites over the Internet. Here, we define the "bundle" to describe a set of multimedia streams produced by multiple sensors of different modalities from the same sender. 


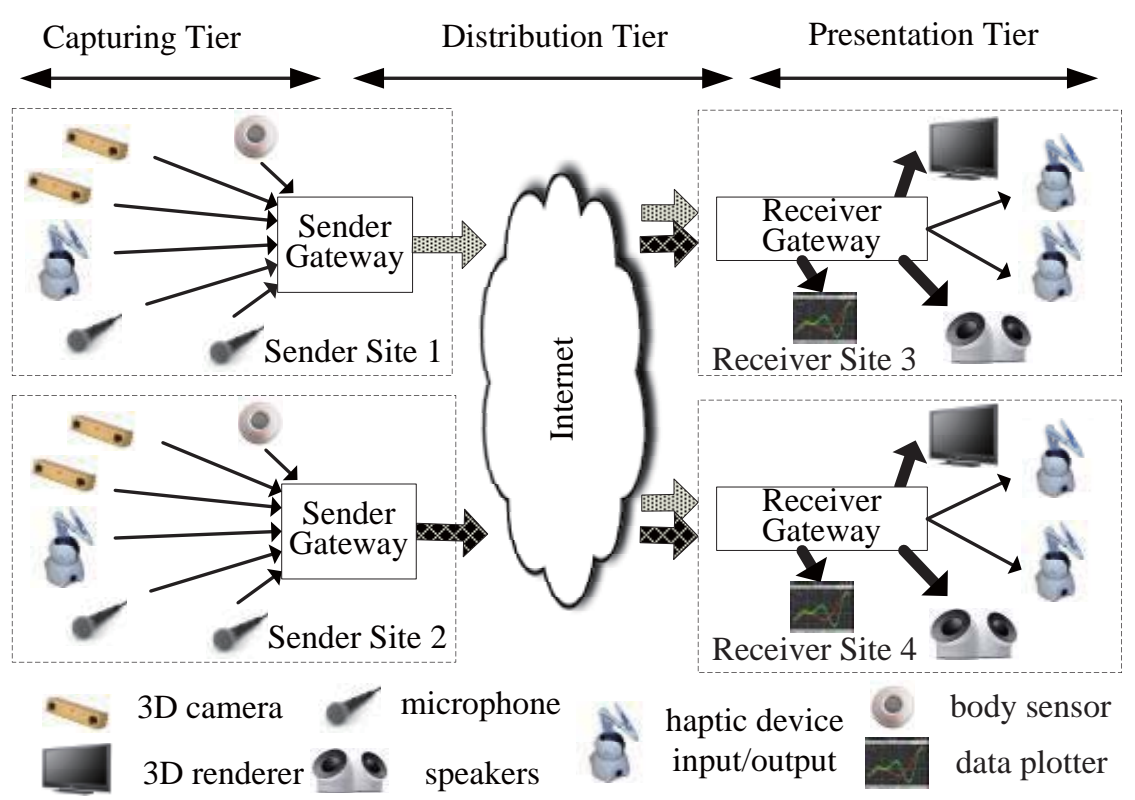

Figure 1.2: Multi-modal multi-stream configuration in multi-site teleimmersive systems.

- Presentation tier. At the receiver sites, the multimedia streams are decoded and played at the corresponding output devices: 3D video displays, multi-channel speakers, and etc.

\subsection{Motivations}

To provide seamless collaboration similar to a face-to-face interaction, an ideal teleimmersive system should distribute the multimedia signals with minimal latency, while preserving their signal intelligibility and time synchronization. However, current best-effort Internet and system computation constraints can inevitably downgrade the overall teleimmersive system quality: an out-of sync multimedia presentation, an imperfect intelligibility of multimedia signals, and a degraded interactivity.

Multimedia synchronization alone is already a challenge in the teleimmersive system. Various system components (e.g., encoding and packetization, communication channel, decoding and presentation, and etc.) belonging to different tiers of the teleimmersive pipeline architecture incur unequal transmission or computation latencies at different multi-modal media frames that are captured at multiple sender sites. This leads to heterogeneous synchronization skews (i.e., the difference of one-way latencies), a violation of time dependencies within and across multimedia sensory streams. Without the collaborative synchronization control across the three teleimmersive tiers, skews introduced in one tier can be propagated to and exacerbated in the next tiers. 
In addition, due to the major advancements of multimedia devices, distributed computing and network technologies, and due to the drop in cost in putting these systems together, the next-generation multimedia systems, such as teleimmersion, are awaiting the scalability of sensory devices, the increased heterogeneity and diversity of media modalities, and the flowing populations of end users. The multimedia synchronization problem can become far more complicated, in terms of concepts and protocols, than the traditional synchronization models, which only take into account limited number of media modalities and sensory streams. Most previous work in the past 10 to 20 years only manage to perform synchronization controls in a multimedia system with a single audio and a single video stream (a detailed survey of synchronization studies will be presented in Section 2.1).

People can have different tolerance and exhibit heterogeneous behaviors to multimedia synchronization quality in various teleimmersive applications. Depending on the user activities, some systems may demand perfect synchronization, while skews in other applications can be negligible. The end user experience should be addressed appropriately in guiding the multimedia synchronization controls.

The human perception is not dominated by the quality of the multimedia synchronization alone in the real-time interactive teleimmersive applications. The system interactive quality and the media signal intelligibility also impact the user satisfactions, and both qualities are affected by synchronization controls. The computation and synchronization control overhead and the Internet latency contribute to the one-way media end-to-end delay (EED) between the capturing device at the sender and the presentation devices at the receiver, which directly decides the interactive quality. The unavailability of media information as the result of synchronization adaptations and Internet degradations (jitter and losses) affect the intelligibility of media signals. Along with the synchronization quality, the media signal intelligibility and the interactive quality have been evaluated individually by respective objective metrics. But none of these metrics is able to successfully represent their combined impact on the overall subjective human perception, which in turn, can affect the synchronization adaptations.

All the above considerations motivate me to investigate the synchronization problems related to the multi-modal media distribution and the human factors in the real interactive teleimmersion. 


\subsection{Synchronization Demands in Teleimmersion}

\subsubsection{Types of Synchronization Demands}

Due to the multi-site multi-sensory configurations, four types of synchronization demands are needed in the next-generation teleimmersive systems, and the general distributed interactive multimedia settings. The four demands form a layered hierarchy, where each synchronization layer from bottom up is depicted in Fig. 1.3.

1. Intra-stream synchronization, which prescribes the synchronous presentation of media frames within each sensory stream at the receivers, according to their original captured timeline at the multimedia sensors. Due to the continuous media nature of teleimmersion, a mis-synchronization in this layer can cause temporal media distortion (e.g., image jerkiness or audio pitch).

2. Intra-media synchronization, which presents the synchronization of sensory streams from multiple media devices of the same media modality (e.g. microphone array or multi-camera array) within a media bundle. A synchronization skew in this layer can violate their spatial correlations during a media presentation (e.g., a visual mismatch between two multiview images).

3. Intra-bundle synchronization, which describes the synchronization of multiple media modalities within a bundle. This layer evaluates the timing consistency across different media modalities. A most studied example is the audio-visual lip synchronization ${ }^{1}$.

4. Intra-session synchronization, which can be either inter-sender or inter-receiver synchronization within a teleimmersive session. An inter-sender synchronization represents the in-sync presentation of media bundles from multiple senders at the same receiver. A mis-synchronization may lead to the confusion of the user located at the receiver site in a highly collaborative activity. An example is a collaborative basketball scenario, where a defensive player (at the receiver site) is interacting with two offensive players (both at sender sites). The temporal synchrony between the offensive players is critical to the perception of the defensive player. On the other hand, an inter-receiver synchronization describes the synchronization of media bundles from the same sender site to multiple receivers. An out-of-sync presentation can cause unfairness when multiple people at different receiver sites require

\footnotetext{
${ }^{1}$ Previous studies $[7,8,9]$ usually combine the intra-media and intra-bundle synchronization demands into a single layer called the inter-stream synchronization within a media bundle, mainly due to the limited number of sensory streams for each media modality (usually only one stream per media modality). In teleimmersion and other next-generation multimedia systems, the scalability of media devices of each media modality and the emergence of new media devices of different media modalities pose different perspectives on intra-media and intra-bundle synchronization demands, and hence, should be addressed separately.
} 


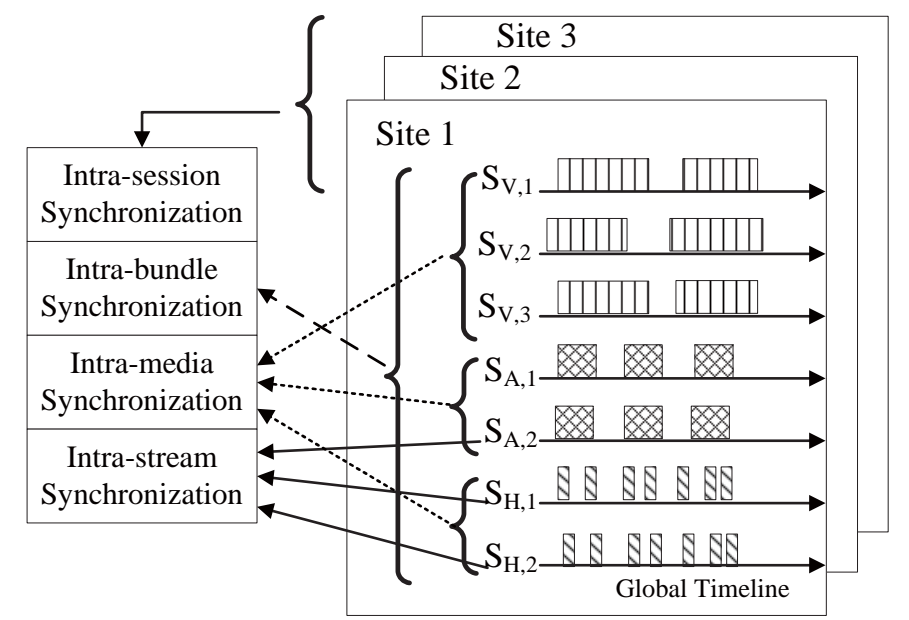

Figure 1.3: Four types of synchronization demands. $s_{i, j}$ denotes each sensory stream, where $j$ is the stream index, and $i=$ ' $\mathrm{V}$ ', 'A' and 'H' represent the video, audio, haptic streams, respectively.

a timing privilege to conduct an activity. For example in a remote education application, students at different receiver sites are racing to answer a question asked by an instructor at the sender site.

\subsubsection{Origin of Synchronization Skews}

For both two-site and multi-site teleimmersion, the heterogeneous latencies of the multi-modal media data incurred at the sender capturing, distribution, and receiver presentation tiers all contribute to the skews in different synchronization demand layers. Synchronization control schemes cannot be properly executed without understanding the origin of these skews.

\section{- Capturing Tier}

Due to the constraints of system resources, the heterogeneity of the computation overhead for media encoding and processing is the key factor causing the synchronization skews in the capturing tier. Fig. 1.4 depicts a comparison of the computation time for two 3D video streams (at a spatial resolution of 320x240 encoded in Berkeley codec [10]) and one audio stream (at a sampling frequency of $16000 \mathrm{~Hz}$ in Speex codec [11]), which capture the same physical environment at the same time. Each multimedia sensor (camera and microphone) employs its own specialized computation machine (with a modern dual-core CPU) during the measurement. Three observations can be made from the figure.

First, a single media stream may demand unequal computation overhead over time (e.g., the video stream $s_{V, 1}$ in Fig. 1.4). This is because the captured physical object does not stand still, so different media 


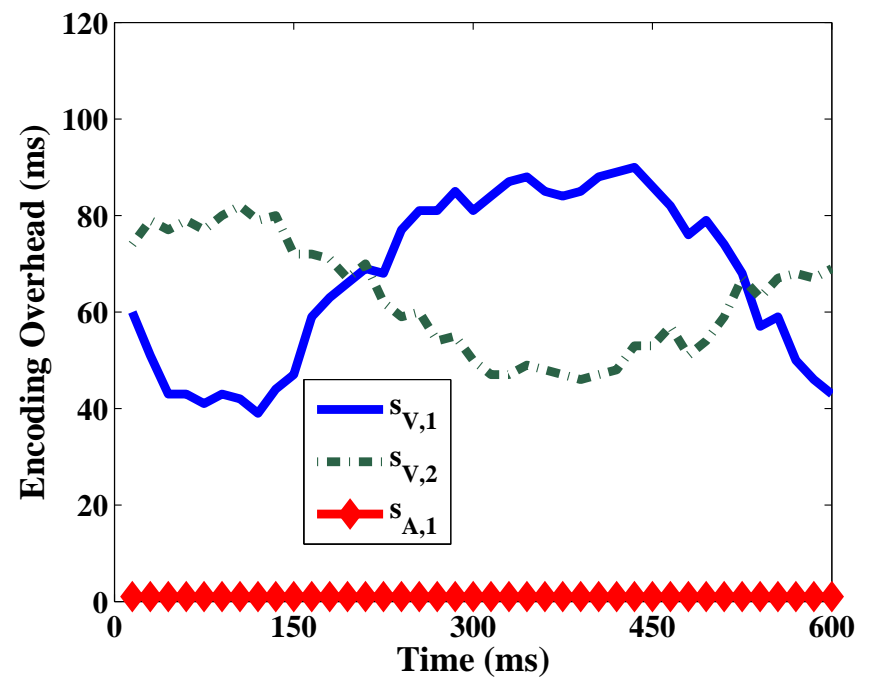

Figure 1.4: Computation time for two video streams $s_{\mathrm{V}, 1}$ and $s_{\mathrm{V}, 2}$, and one audio stream $s_{\mathrm{A}, 1}$.

frames within the sensory stream can carry different amounts of media information and require different computation time. This variation introduces the intra-stream synchronization skew.

Second, multiple media streams of the same media modality can also experience heterogeneous computation overhead at the same time (e.g., the two video streams $s_{V, 1}$ and $s_{V, 2}$ in Fig. 1.4), because they may capture the same physical object from different directions with different amounts of media data. This heterogeneity will affect the intra-media synchronization.

Third, two media modalities may require very different computation demands (e.g., the audio and 3D video in Fig. 1.4), which will contribute to the intra-bundle synchronization skew.

The above observations prove a demand for appropriate synchronization control schemes in the capturing tier, for the purpose of preventing the skews to be generated, and hence, propagated to the distribution and presentation tiers.

\section{- Distribution Tier}

In the two-site teleimmersive systems, the Internet delay and jitter over time are the main cause of the multimedia skews in various synchronization layers. In addition, the sensory streams can have heterogeneous Quality-of-Service (QoS) requirements, because different streams employ their own protocols and adaptation algorithms at the sender gateway due to their diverse characteristics. This heterogeneity will inevitably result in different transmission overhead which will contribute to the synchronization skews.

In a multi-site setting, an overlay connectivity can be deployed (Fig. 1.5), where a sensory stream may be relayed though some intermediate sites (i.e., sites that relay media streams to other sites) during its 


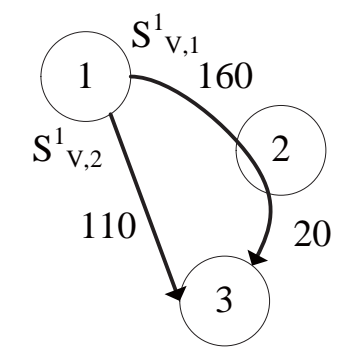

(a) Problem of intra-media synchronization

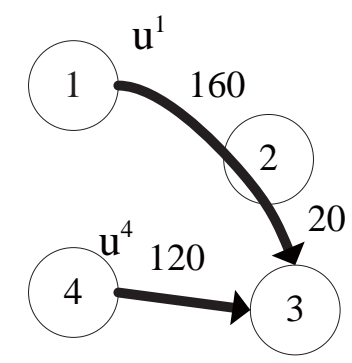

(b) Problem of intra-session (inter-sender) synchronization

Figure 1.5: Overlay topology and issue of intra-media and intra-session synchronization. The sites are represented by circles and the paths are indicated in curves. $s_{\mathrm{V}, j}^{x}$ denotes the video stream from sender site $x$, where $j$ is the stream index, and $u^{x}$ represents the media bundle from sender site $x$. The numbers by the paths represent the Internet average latency (unit: $\mathrm{ms}$ ).

distribution to the receiver. This will potentially exert three impacts to the multimedia synchronization.

First, the aggregate jitter over multiple links can contribute to the overall latency variation which will further impact the intra-stream synchronization.

Second, multiple multi-modal sensory streams within a media bundle from the same sender site may follow different paths to reach the same receiver site [12]. The heterogeneity of the Internet latency incurred on multiple path options can impact both intra-media and intra-bundle synchronization. For example, Fig. 1.5(a) shows the two video streams $s_{\mathrm{V}, 1}^{1}$ and $s_{\mathrm{V}, 2}^{1}$ from the sender 1 use different paths to reach the receiver at site 3 , thus introducing the intra-media synchronization problem.

Third, the mix of single-hop and multi-hop paths from the same sender to multiple receiver sites, or from multiple sender sites to the same receiver, can increase the likelihood of both inter-sender and inter-receiver mis-synchronization within the session. Fig. 1.5(b) demonstrates the case of intra-session (inter-sender) synchronization problem where the media bundle $u^{1}$ and $u^{4}$ from sender sites 1 and 4 use different paths to reach the receiver site 3 .

While we have no control over the Internet delay and jitter characteristics, we are able to monitor the network traffic statistics in real time, and periodically check which path will introduce an unbounded multi-layer synchronization skew during the media distribution. This leads to the design problem of a synchronized overlay for multi-modal multi-stream distribution in multi-site teleimmersion.

\section{- Presentation Tier}

The synchronization skews are determined by the one-way latency difference of the time-correlated media data at their presentation, and the latency is directly decided by the receiver buffer size. Existing 
studies $[13,14,15,16,17]$ usually realize multimedia synchronized presentation by inserting buffering latency to compensate for the skews which have been introduced so far during the media computation and distribution, and to decide the time that the media data are scheduled to be presented. So these schemes are often named media presentation scheduling. In the next section, we will argue that the receiver media presentation scheduling is still a challenge due to the impact of the human perception.

\subsection{Synchronization and Human Perception}

The performance of synchronization control schemes affect the synchronization quality, the media signal intelligibility and the interactive quality, which represent three orthogonal dimensions of the multi-faceted streaming media quality. The dynamic Internet quality and the resulting synchronization adaptations can also cause consistent status changes of the streaming media quality, leading to the flicker effect. The streaming media quality and the flicker effect are the two different quality factors that combinedly impact the overall human perception in the interactive teleimmersion. Hence, their interactions must be addressed carefully in designing the synchronization control algorithms, for the purpose of maximizing the end user satisfactions.

\subsubsection{Impact of Synchronization Controls}

\section{- Streaming Media Quality}

Synchronization Quality. Various multimedia synchronization control schemes perform by controlling the processing and distribution overhead in each teleimmersive system tier, and bounding, in each synchronization demand layer, the resulting skews within the synchronization threshold (i.e., the upper bound of skews). During these control adaptations, a synchronization reference is a must for other media data to be synchronized against.

Media Signal Intelligibility and Interactive Quality. Both quality dimensions are also affected by the synchronization controls. For example, the presentation scheduling with receiver buffer adaptation has been heavily studied to reduce the negative impacts of Internet imperfections on the multimedia synchronization quality. But unfortunately, existing presentation scheduling algorithms create a tradeoff between the media signal intelligibility and the interactive quality. For example, we use a larger receiver buffer to enhance the availability of media packets at their scheduled presentation by smoothing greater Internet jitter. This will result in an increased media frame rate or reduced media content distortion, which can improve the media 
signal intelligibility. But as a side effect, the interactive quality will be sacrificed due to the prolonged media EED. On the other hand, we can properly decide the receiver buffer size in order to reduce the skew to minimum, but this will affect both the media signal quality and the interactive quality. Because of the multi-dimensional quality tradeoff, it is very difficult for the presentation scheduling algorithms to decide the best operating status of the streaming media quality during the presence of Internet degradations.

\section{- Flicker Effect}

Synchronization algorithms can periodically update online based on Internet and computation system statistics. At each update, the multimedia system will renew the target operating status of the synchronization quality, signal intelligibility and interactive quality. On the other hand, the Internet can exhibit dynamic behaviors. A sudden change of the network condition without timely synchronization control update will also change the status of three quality attributes. The transition of the three-dimensional streaming media quality from one status to another introduces the flicker (i.e., the gap between two streaming media quality statuses). A large flicker can interrupt the smoothness of the interactive multimedia presentation.

\subsubsection{Impact of Human Perception}

\section{- Streaming Media Quality}

Synchronization Quality. Humans can feel annoyed at an out-of-sync multimedia presentation in any synchronization demand layer. Hence, to achieve the multimedia synchronization controls, it is critical to select the synchronization reference and prescribe the synchronization threshold that will not cause noticeable perceptual degradations. However, both the synchronization reference and the synchronization threshold can vary in different activities.

1. Activity-dependent synchronization reference. As a rule of thumb, the synchronization reference is usually selected as the one which has the largest contribution to end user perception. For example, previous studies $[13,15,17,18,19]$ choose the audio stream as the reference to all other streams, because people are more sensitive to the audio signal degradation. This is understandable because past commercial applications are mostly single-functional, and are limited to $2 \mathrm{D}$ video conferencing or $2 \mathrm{D}$ on-demand streaming, where mostly a single audio and a single video stream are considered. The synchronization reference in these studies is prescribed regardless of the activities and media application functionality. However, in the next-generation multimedia systems like teleimmersion, 
a receiver may present scalable multi-modal multi-streams from multiple media sources. Hence, we need a systematic methodology to evaluate the importance of each sensory stream to the user perception. In addition, these multimedia systems will be multi-functional, and the same distributed multimedia infrastructure will be deployed for various applications. The activity-dependent usage of multimedia systems will require that synchronization references change throughout the activities, which should be unambiguously reflected in the next-generation synchronization framework.

2. Activity-dependent synchronization threshold. Previous studies $[20,21,22]$ have identified the threshold and the impact of an audio-visual lip skew in video on-demand and conferencing systems, and their experiments are conducted on both PC and mobile displays. However, due to the multifunctional nature of teleimmersion and other multimedia applications, humans can also exhibit different tolerance on the same skew in heterogeneous activities. An accurate synchronization threshold cannot be assessed without real subjective evaluations.

Hence, a systematic methodology is highly appreciated to prescribe the synchronization reference and synchronization threshold based on heterogeneous functionalities and performed activities of different multimedia applications.

Media Signal Intelligibility and Interactive Quality. A poor media signal intelligibility can contribute to the misunderstandings and confusions at end users during the media presentation. Generally, people prefer media signals with high frame rates to guarantee the presentation smoothness, and less distorted contents for the best media clarity. On the other hand, a degraded interactive quality with a long media EED can interrupt the shared activities among the end users and may introduce doubletalks during their conversation in extreme cases.

While the human perceptual impacts of the three streaming media quality dimensions have been evaluated individually, no existing study is able to capture their combined impact under the activity heterogeneity and the multi-dimensional quality tradeoff (detailed comparisons will be presented in Section 2.3). Hence, it is difficult to perform synchronization adaptations and to find a best control status to realize the best user satisfactions in an interactive multimedia system, without real human perceptual feedback online.

\section{- Flicker Effect}

Generally speaking, larger and more frequent flickers can introduce greater discomfort to end user perception. Hence, we should design synchronization controls such that flickers can be minimized during the system run time under control adaptations and potential Internet quality changes. 


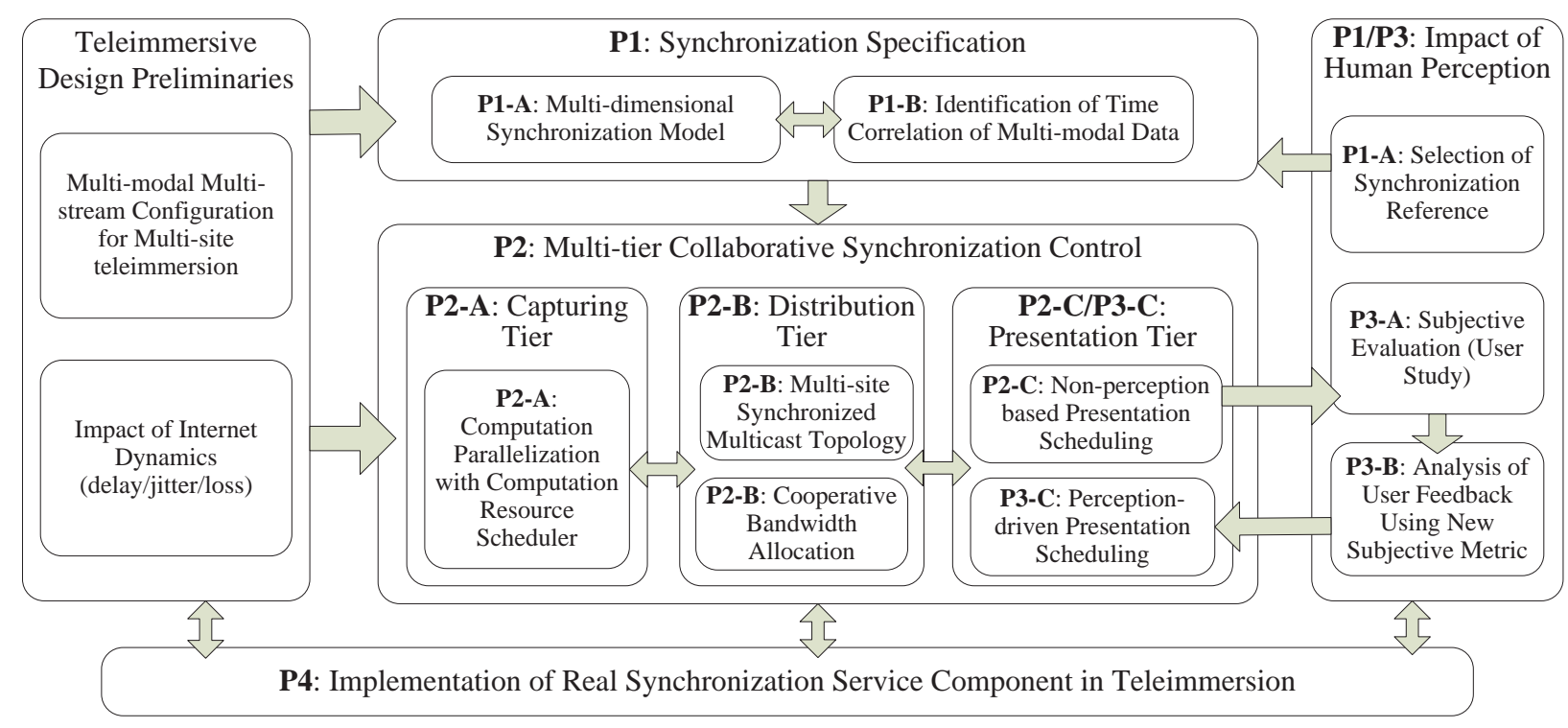

Figure 1.6: Overview of this dissertation.

\subsection{Thesis Problem Statement}

The goal of this dissertation is to achieve a multi-site distributed interactive teleimmersive system with multi-modal multi-stream synchronization and high user satisfactions. The overall design can be divided into multiple key problems. Their interactions are described in Fig. 1.6.

\subsubsection{P1: Synchronization Specification}

In P1, we investigate the required specification model to characterize the new synchronization problems in the next-generation multimedia architecture. These specifications will be used extensively in the whole synchronization control framework in this dissertation.

\section{- P1-A: Multi-dimensional Synchronization Model}

The first problem we are interested is the model for describing the multimedia synchronization problems. Previous studies [18, 19, 23, 24] propose classification methodologies based either on the type of synchronization demands [18, 19] (e.g., the four synchronization layers discussed in Section 1.3.1), or on the locations where synchronization controls are performed over one specific layer [23, 24] (e.g., controlling the intra-media skew in the three teleimmersive tiers of Section 1.1). In the teleimmersive system, skews of one synchronization layer in a location (tier) can affect those of other layers in future locations (tiers). This new and complex contextual dependency encourages a model that integrates both demand-based and 


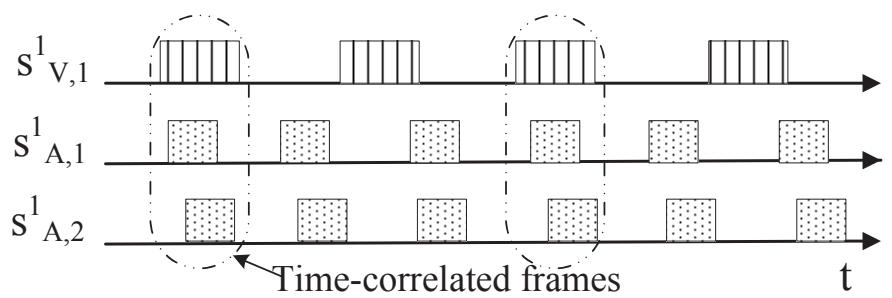

Figure 1.7: An example of time-correlated media frames for one video and two audio streams.

location-based classifications, with one type of classification methodology representing one orthogonal dimension. This is especially needed when the next-generation systems see a rising complexity of hardware configurations and an increasing number of pipeline components (tiers) involved in the overall media distribution architecture.

The dimension of activity-dependent synchronization reference hierarchy should also be added to be the synchronization model, for describing heterogeneous human perceptual impacts in diverse teleimmersive applications. Due to the multi-layer synchronization demands, it is not possible to use a single synchronization reference to represent the whole layered hierarchy. Each synchronization layer must identify its own reference based upon the media contribution to end user experience.

\section{- P1-B: Identifying Time Correlations of Distributed Media Sensors}

The global timestamp of media frames from distributed sensory devices at each sender site is important for multi-modal multi-stream synchronization in teleimmersion. Oftentimes, we need to identify at each sender site a sequence of time-correlated multi-modal media frames, which capture the sender physical space at approximately the same time (Fig. 1.7), but from different perspectives. These time-correlated media frames are important for the multimedia synchronization, because (1) they must be presented synchronously at the output media devices at the receiver sites to minimize media presentation distortions, and (2) their time dependencies facilitate the evaluation of multi-layer synchronization skews in different system locations.

However, different media sensors, even at the same sender site, can capture the media frames independently without hardware synchronization mechanism. The resulting time misalignment of these media frames has prohibited us to tell exactly which set of multi-modal media frames (one frame from each sensory stream) is really time-correlated without a systematic decision methodology. In addition, we cannot guarantee each sensory device produce a periodic media signal due to the system constraints. These issues complicate the problem of accessing global time correlations in teleimmersion. 


\subsubsection{P2: Multi-tier Collaborative Synchronization Control}

To achieve the synchronous presentation of media data at the receiver output devices, we perform collaborative synchronization controls in all three system tiers, and minimize the synchronization skews in each tier. Note that all the problems in P2 are non-perception based, meaning that they can be solved without the aid of subjective evaluation results. We will leave the perception-based problems to P3.

\section{- P2-A: Capturing Tier Control}

In the capturing tier, we aim to minimize the heterogeneity of computation overhead for multi-modal multi-streams that would otherwise introduce the skews in multiple synchronization layers. A common approach is to perform encoding/processing parallelization, by devoting more computation resources to reduce the overhead incurred at computation-intensive media streams, in order to reduce the gap from those with negligible computation time. However, existing parallelization methods usually focus on the media encoding speedup without considering varying the scheduled amount of computation resources online [25, 26] based on real media computation demands. These methods cannot guarantee bounded skews within and across sensory streams.

\section{- P2-B: Distribution Tier Control}

In the distribution tier, our goal is to design an overlay topology for multi-site teleimmersive applications that can bound the skews during the media distribution. The one-way latencies should also be minimized for all sender and receiver pairs in order to maximize the teleimmersive interactivity. Note that multiple receivers may request different subsets of sensory streams from a same sender site, due to their individual interests. Previous studies $[12,27,28,29,30,31]$ on the multicast overlay oversimplify the problems by either assuming the homogeneity of the media data requested at different receiver sites, or by overlooking the interactivity and synchronization aspects of the overlay problem. They cannot directly be applied to solve the synchronization problem of the interactive multi-modal teleimmersive distribution.

The scalability of bandwidth-savvy multi-modal multi-streams contribute to the network burdens at end systems and intermediate sites. At an insufficient bandwidth availability, media packets can experience unexpected large congestion jitter which will impact the multi-layer synchronization skews. Media signal intelligibility can also be downgraded because of the potential congestion losses. Hence, a dynamic bandwidth resource allocation scheme is a must to minimize the the network congestions in order for successful multi-site media distribution. 


\section{- P2-C: Presentation Tier Control}

We study the non-perception based media presentation scheduling approach in $\mathrm{P} 2$, which performs dynamic buffer adaptation of each sensory stream within the teleimmersive session based on Internet jitter, and at the mean time, achieves the demand of media signal intelligibility and synchronization quality at the minimal latency. Different from previous work [17, 32, 33], which focus on lip synchronization of a single audio and a single video stream in the conferencing or on-demand video systems, each sensory stream in teleimmersive applications has to decide its individual buffer size in a hierarchical fashion, by consulting the corresponding layered synchronization references.

\subsubsection{P3: Synchronization and Human Perception}

In P3, we are interested in evaluating the impact of synchronization controls on overall user subjective satisfactions in diverse teleimmersive activities. The generalized subjective perceptual feedback, in turn, is employed to drive the synchronization controls in interactive multimedia applications in our study.

\section{- P3-A: Design of Subjective Evaluation Methodology}

We conduct a user study to evaluate the human perceptual quality as the result of synchronization control adaptations. Because the subjective study cannot be successful without a careful design of the evaluation methodology, we aim to address the following design issues. We focus on evaluating the multi-dimensional streaming media quality in P3-A.

First, the selection of teleimmersive activities is the key to evaluate human perceptual impacts. We prefer activities that are representative. Because subjective studies are very time-consuming in practice, we have to understand people's heterogeneous demands on synchronization quality, media signal intelligibility and interactive quality from limited numbers of selected tests. These activities must also be simple. The complications of performing and evaluating complex activities in user studies may bias the real impacts on end user experience.

Second, we have to design a subjective test that is controllable and repeatable. It is usually not possible to evaluate an online test in the real interactive teleimmersive testbed, where user study participants (i.e., the subjects) themselves are involved in the activities. The reason is that we are unable to control the progress of the online test, and the resulting irreproducible evaluation results may lack creditability.

Third, multiple media samples are needed to capture different multi-dimensional streaming media quality values that are most likely to appear during the synchronization controls. To achieve this, objective 
metrics must be identified to describe the characteristics of each quality dimension. The whole objective quality space also needs to discretized in order to reduce the evaluation process.

Fourth, we demand a best subjective rating method. ITU-T BT. 500 [34] and P.910 standards [35] have defined multiple methods including the absolute category rating (ACR), the degradation category rating (DCR), and the comparative category rating (CCR). Different rating methods, however, have their own pros and cons (details will be discussed in Section 2.2). The best method should be decided based upon the characteristics of our user study.

\section{- P3-B: Analysis Using New Subjective Metric}

To present the user ratings (votes) from the subjective evaluation, a satisfactory subjective metric is also needed. One approach is to compute the votes of all subjects, and the resulting average is called the mean-opinion-score (MOS). ITU-T BT.500 and P.910 have defined multiple versions of the metric, including MOS, CMOS (comparative mean-opinion-score) and DMOS (degradation mean-opinion-score), that represent the subjective metric of ACR, CCR and DCR respectively. However, the major problem of this set of subjective metrics is that the average score can conceal the variations of user votes. The variations are very common in evaluating the interactive multimedia applications [36,37], where the overall human perception is concurrently dominated by multiple streaming media quality attributes. Hence, a new subjective quality metric is needed, and the goal of our perceptual analysis is to find a mapping from the objective quality space characterizing each streaming quality dimension to the subjective quality space describing their combined perceptual impacts.

\section{- P3-C: Design of Perception-driven Media Presentation Scheduling}

By generalizing the streaming media quality mapping based on the new proposed subjective metric, we study the perception-driven media presentation scheduling algorithm to maximize the end user experience. On one side, this means we would need to achieve a control operating status with the best streaming media quality. But due to the interaction and tradeoff among its three quality dimensions, we will show that only a set of local-optimal streaming media qualities can be accessed. On the other side, the flicker effects arising from the network condition variations and the system adaptations should also be minimized in our perception-driven design.

Previous papers on VoIP and video conferencing [38,39] simplify the perception-driven adaptation problem without considering the multi-dimensional quality tradeoff. Our previous studies $[36,37,40]$ focus on VoIP, and use subjective findings to address the tradeoff between the audio signal intelligibility and the 
interactive quality. None of these studies is able to consider the interaction and tradeoff of quality attributes in all three dimensions, and to minimize the impacts of flicker degradations on human perception in the interactive multimedia applications.

\subsubsection{P4: Implementation of Synchronization Service Component}

We will realize the synchronization specifications, protocols and algorithms in the real TEEVE testbed. This implementation is expected to consistently function well under Internet dynamics, and under the scalability and increasing diversity of multimedia system configurations.

\subsection{Research Approaches}

The following procedures illustrate our approaches of solving the synchronized distribution problem for high-quality interactive teleimmersive systems. We refer to Fig. 1.6 for describing our research approaches.

Step 1. We configure the teleimmersive system with multiple 3D video, audio, haptic, body sensory streams, and etc. To perform repeatable experiments under the same system setting, but with different algorithm plugins and in diverse network conditions, we emulate a real teleimmersive setting, prerecord these sensory streams, and replay them in PlanetLab sites using either TCP or UDP. Different streams are sent according to their real frame size and arrival time at the sender gateway. We study the media traffic characteristics, and classify Internet traces into different categories based on the network delay, jitter and loss statistics. This will allow us to conduct and analyze the experiments in each category.

Step 2 (P1). We propose a multi-dimensional synchronization model incorporating the synchronization demand, the synchronization location, and the synchronization reference hierarchy as each orthogonal dimension. We present a systematic methodology to prescribe the hierarchical references for each synchronization demand layer, based upon the multimedia application functionality and end user interests. We also develop an analytic model to identify the time correlations of multi-modal media data from multiple sensory devices based on their arrival time at each sender gateway.

Step 3 (P2). We design the collaborative synchronization control framework. In the capturing tier, we present a computation resource scheduling algorithm for media computation parallelization, by predicting the processing/encoding process and dynamically allocating the number of required computation resources. In the distribution tier, we use a heuristic algorithm to solve the synchronized multicast overlay design problem, which in nature, is NP-hard. When the bandwidth resources are not sufficient, our cooperative band- 
width allocation algorithm is activated by prioritizing media streams (from different distributed sources) at sender and intermediate sites. In the presentation tier, we achieve the final multimedia synchronization by adaptive presentation scheduling of media data based on the distribution of their arrival time. All the studies in $\mathrm{P} 2$ are non-perception based.

Step 4 (P3-A). We identify objective metrics characterizing both the media streaming quality and the flicker effect. For the streaming media quality, we propose a systematic methodology to demonstrate that existing subjective metrics are not sufficient to describe the subjective comparison results where the tradeoff of multiple quality dimensions is involved. We evaluate both conferencing and rock-paper-scissor applications, so as to capture heterogeneous human perceptual behaviors. We generalize the subjective results using support vector machine [41]. We conclude that there is a demand for a new metric to interpret the subjective comparison results and to guide the system adaptations. We focus on the two-site teleimmersive applications in the subjective evaluations.

Step $5(\mathbf{P 3}-\mathbf{B} / \mathbf{C})$. We propose a new subjective metric to capture the contradicting opinions among subjective votes under multi-dimensional streaming media quality tradeoff, based on the hypothesis testing. By employing the learned regression model at run time, we propose a perception-driven media presentation scheduling algorithm which can dynamically and consistently select the control operating status that leads to the best user perception of the streaming media quality and the minimal flicker degradation, even under unseen network conditions. We use a perception-based genetic approach to decide this best system operating status. We perform statistical flicker estimation based upon previous Internet records in order to guide the flicker minimization. Again, we only study the two-site teleimmersive system here.

Step 6 (P4). We implement a real service component for multi-modal multi-stream synchronization on top of the existing streaming as a service (SAS) kernel [42] in the real TEEVE testbed. We will show its effectiveness by emulating Internet degradations (delay/jitter/loss) between the teleimmersive sites. Due to unsolved firewall issues between Illinois and other testbeds in Texas and Berkeley, we are unable to evaluate across these satellite testbeds by the time we finish this dissertation.

\subsection{Research Contributions}

The contributions of this dissertation can be summarized in the following two aspects:

First, the key novelty of this dissertation is to highlight new synchronization requirements of teleimmersion and other next-generation interactive distributed multimedia systems, and address the new demands in 
next-generation synchronization modeling, algorithmic and protocol framework. We would like to point out that the underlying assumptions of the past synchronization studies are far from sufficient as our multimedia environments are getting richer in terms of multi-modal devices, more powerful in terms of computing power, and faster in terms of the high network inter-connectivity. The dissertation serves to encourage the research and industrial community to rethink of new synchronization models, concepts, specifications, mechanisms and protocols, in order to guide the future synchronization studies and develop new industrial standards.

Second, we drive the synchronization controls based on the real human perceptual feedback from extensive subjective evaluations. To the best of our knowledge, our dissertation is the first study to use the real subjective experiment data to address the interaction and tradeoff of the synchronization quality, media signal intelligibility and interactive quality in an integral and systematic method. Previous studies can only study the human perceptual quality of one or multiple quality dimensions without addressing their inherent tradeoff (discussions see Section 2.3). We argue that existing subjective metrics are incompetent in describing the contradicting opinions which are highly likely in the distributed interactive multimedia applications, and that existing ITU standards on perceptual quality models based on these metrics should be consulted with cautions. New standards based on a redesign of subjective evaluation and analysis methodology are imperatively demanded.

\subsection{Dissertation Outline}

Chapter 2 presents a complete survey of previous related work that has been done on multimedia synchronization and human perceptual quality for interactive multimedia. Chapter 3 discusses new synchronization specifications covering the multidimensional synchronization model and the adaptive time correlation identification algorithm (P1). Chapter 4 proposes the collaborative multi-tier synchronization control framework (P2). Chapter 5 conducts the subjective evaluations on both teleimmersive conferencing and rock-paperscissor gaming applications, analyze the user feedback based on new proposed subjective metric, and use the generalized perceptual results to guide online receiver presentation scheduling (P3). Chapter 6 presents the implementation details of the synchronization service component in the real teleimmersive systems (P4). Chapter 7 concludes the dissertation. 


\title{
1.9 Table of Abbreviations and Notations
}

\author{
List of Abbreviations in Chapter 1: \\ TEEVE Teleimmersive Environment for EVErybody \\ ITU International Telecommunication Union \\ MOS Mean-Opinion-Score \\ DMOS Degradation MOS \\ CMOS Comparative MOS \\ ACR Absolute Category Rating \\ CCR Comparative Category Rating \\ DCR Degradation Category Rating \\ QoS Quality of Service \\ QoE Quality of Experience \\ SVM Support Vector Machine \\ W.L.O.G Without Loss of Generality \\ TCP Transmission Control Protocol \\ UDP User Datagram Protocol \\ IP Internet Protocol \\ VoIP Voice over Inter Protocol \\ EED End-to-End Delay \\ MPS Media Presentation Scheduling
}

\section{List of Notations in Chapter 1:}

$i \quad$ Index of media modality

j Index of sensory stream

$s_{i, j} \quad$ Sensory stream of media modality $i$ and stream index $j$ 


\section{CHAPTER 2}

\section{RELATED WORK}

This chapter surveys existing research studies and industrial standards on multimedia synchronization specifications, algorithms and protocols, as well as the evaluations of human perceptual quality in the multimedia systems. We will discuss their applications and drawbacks in the teleimmersive studies.

\subsection{Historical View of Synchronization Studies}

Ever since the invention of the world's first telephone in 19th century, the advancement of multimedia applications have drastically changed human life and behaviors, and have introduced new demands for multimedia synchronization. In this section, we present a historical view of the synchronization research studies with a focus on the continuous multimedia, and discuss how these work are impacted by the development of new interactive multimedia systems.

\subsubsection{Years of Birth: On and Before 1988}

The rise of electronic technologies had given birth to lots of new multimedia applications before the year of 1988. But due to the immaturity of these technologies, only limited studies realized multimedia synchronization as a problem, and mainly they were related to the fidelity or intelligibility of multimedia signals. As one of the oldest and the most important networking protocols, the Network Time Protocol (NTP) was proposed in 1985 to perform computer clock synchronization, and it has been implemented in almost every machines nowadays.

\section{- Historical Background}

Back to the years of 1860 s and 1870s, the telephony device was invented to allow the analog speech transmissions over wired circuits $[43,44]$, thus opening up the new era of multimedia innovations. Fifty years later, both Charles Jenkins from the U.S.A. and John Baird from Scotland demonstrated the trans- 
missions of images [45]. The commercial television set also became available in the late 1920s, and the broadcast TV service has been a commonplace since then. Later in 1960s, AT\&T Bell Labs demonstrated its own picturephone (or videophone) and the first transcontinental call. AT\&T's videophone was able to support a video frame rate of up to $30 \mathrm{~Hz}$ with a bandwidth of $1 \mathrm{Mbps}$ [46]. In 1974, the microphone array (or microphone antenna) technique was invented by Billingsley [47]. In 1986, Kesmai introduced the multiplayer flight combat simulation game Air Warrior, which was the first graphical massively multiplayer online game (MMOG) in the world [48].

Multimedia synchronization was not a major demanded issue in those early years, mainly because the analog audio and analog video signals were usually multiplexed and transmitted in a controlled communication channel [46]. In addition, the successful operations of these application functionalities, and the quality of analog audio and video intelligibility were themselves immature, so they became the first problems to solve. The concepts of these new-born multimedia applications were also just accepted by people, who demonstrated more of a curiosity than an every-day demand.

\section{- The Start of Synchronization Perception Studies}

It was not until the years of 1970s and 1980s that the digital multimedia synchronization was realized as a problem. The invention of the computing machines fostered the development of digital media, while the introduction of the best-effort Internet and TCP/IP protocols brought people's attention to the concept of "jitter". People became interested in how the jitter affected the human perception on the digital media fidelity, and multiple preliminary results were developed to discuss the impact of jitter on the intra-media synchronization of the digital audio. For example, Dannenberg [49] offered several experimental results demonstrating that in one sampling period, only a 200-ns jitter is allowed for 16-bit audio. Similar results could also be found in [50], which recommended a largest jitter of no more than $10 \mathrm{~ns}$.

\section{- NTP: A Clock Synchronization Protocol}

In 1985, David Mills, a professor of the University of Delaware, proposed the first version of Network Time Protocol (NTP), a protocol designed for synchronizing the clocks of distributed computers connected by packet-switched networks like Internet. To synchronize one computing machine (called the client) against the other (called the server), the NTP client computes the round-trip delays by sending a set of UDP packets to the remote server, based on which the clock offset between the machines can be estimated. We assume the time that a packet leaves the client at $t_{1}$ and arrives at the remote server at $t_{2}$ (Fig. 2.1(a)). We also denote the time that the packet leaves the server at $t_{3}$ and returns back to the client at $t_{4}$. All time are 


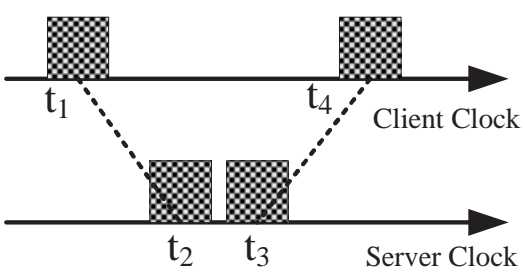

(a)

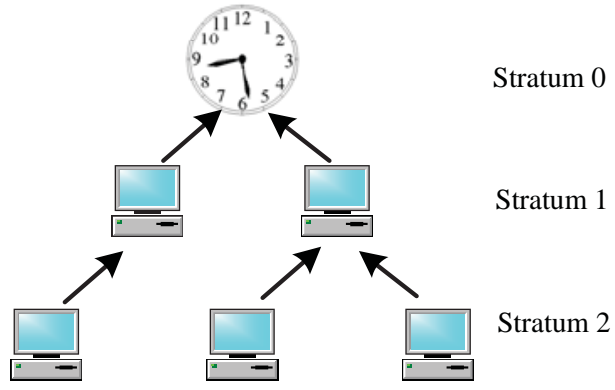

(b)

Figure 2.1: (a) NTP clock offset computation, (b) NTP multi-stratum hierarchy.

measured based on the local clocks. Hence, the clock offset between two machines is:

$$
\delta=\frac{\left(t_{2}-t_{1}\right)+\left(t_{3}-t_{4}\right)}{2}
$$

Because the synchronization approach assumes the round-trip delay symmetry, the clock synchronization accuracy is impacted by the Internet delay variations. To improve the accuracy, one will usually select a NTP server that is close to the client in the real practice. But in general, NTP can only offer a synchronization accuracy in the range of $10 \mathrm{~ms}$ [18]. NTP also considers the scalability of computing machines by adopting a multi-stratum synchronization hierarchy (Fig. 2.1(b)), where machines in a stratum layer $k$ are synchronized to the corresponding servers in the higher stratum layer $k-1$.

\subsubsection{Years of Understanding: 1989-1994}

Owing to the technological advances of the Internet protocols (IP), many Internet-based digital multimedia systems emerged and were commercialized in the late 1980s and early 1990s. Multimedia synchronization became a known and important topic to the research community, and lots of work were done to understand the synchronization problem. These studies covered a broad synchronization area including the classification and specification modeling, subjective perception evaluation and synchronization control algorithms.

\section{- Historical Background}

In 1991, IBM and PictureTel introduced the first PC-based black-and-white video conferencing system [51]. During the same year, DARTnet made another milestone by achieving a transcontinental IP-based video conferencing between the U.S.A and the U.K [52]. In 1992, a real-time virtual multichannel acoustic environment was invented by Gardner based on the microphone arrays [53]. Later in 1994, the application for Computer Supported Cooperative Work (CSCW) was introduced, which could be employed for various 


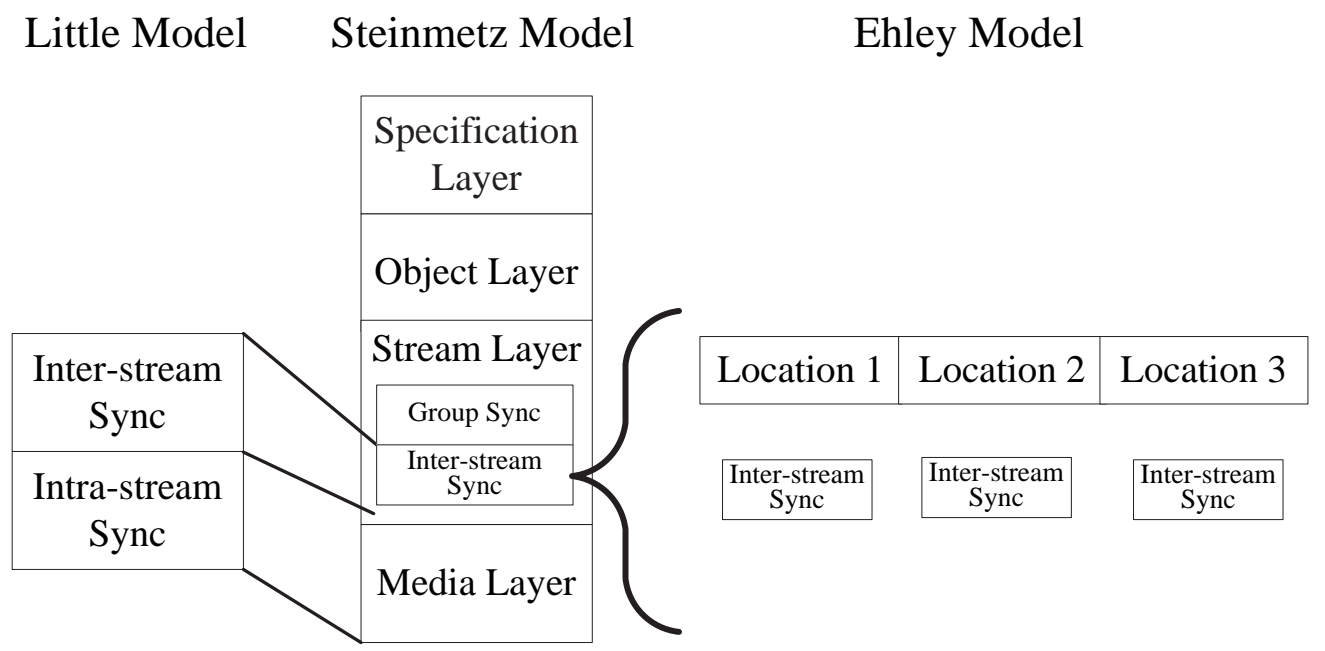

Figure 2.2: Three synchronization classification models.

functionalities including teleconference [54]. The first commercial television program, ABC World News, was broadcasted over the Internet in the same year, based on the CU-SeeMe software [55]. The video ondemand (VOD) service was also started under Cambridge project, offering streaming videos at a bandwidth up to $25 \mathrm{Mbps}[56]$.

The proliferation of new Internet-based multimedia systems and the improvement of digital audio-visual fidelity promoted the researchers to address the synchronization problem. The Internet delay variations between the (single) audio and (single) video streams in both video conferencing and on-demand video systems, and between multiple audio streams in the microphone array setup, exhibited a need for inter-stream synchronization, i.e., synchronization across multiple sensory streams. The development of teleorchestration service and CSCW demanded an in-sync media presentation across multiple distributed receivers or a group of receivers, called group synchronization (same as inter-receiver synchronization). Thus, the multimedia synchronization studies became a heated topic during the years of 1989-1994.

\section{- Synchronization Classification}

To understand heterogeneous demands of multimedia synchronization, a classification model is needed for investigating the structure of synchronization mechanisms and comparing runtime controls that realize in-sync presentation of multimedia data. Lots of classification models were proposed in the years of 19891994, with views from different aspects of the synchronization problem. A complete survey has been done by [18]. Here, we pick and analyze the three classification models that are directly related to our studies. Their interactions are shown in Fig. 2.2. 


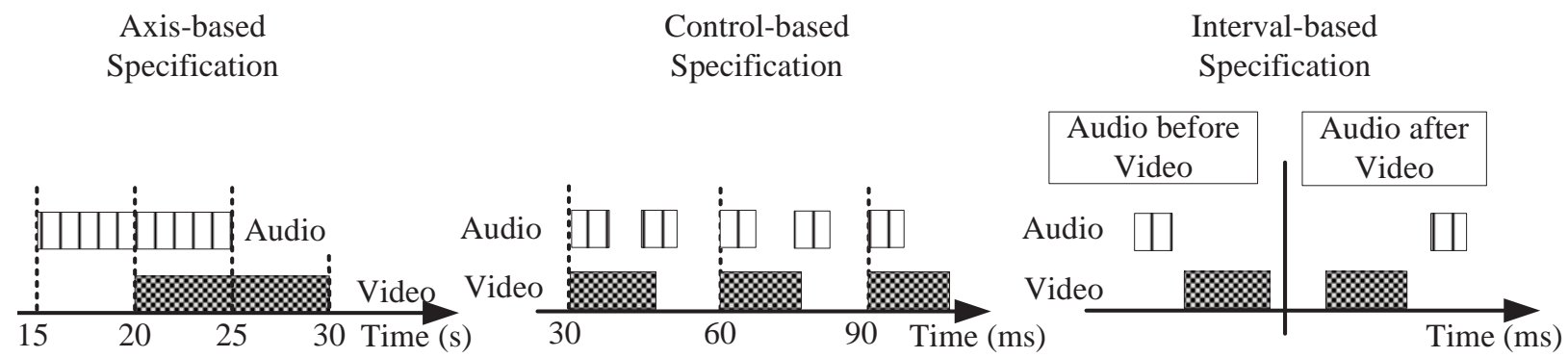

Figure 2.3: Three synchronization specification models.

Little et al's Model [7]. Proposed by Little, Thomas and Ghafoor in 1991, the classification model spans over the synchronization of continuous media frames within a single sensory stream (i.e., intra-stream synchronization) and the synchronization across multiple audio-visual streams (i.e., inter-stream synchronization). Discrete media objects like still images and texts were also investigated in their model.

Steinmetz et al's Model $[8,9]$. Meyer, Effelsberg and Steinmetz presented a more sophisticated synchronization model in 1993, based on the types of synchronization demands. The model is divided into four synchronization layers: (1) media layer, i.e., the intra-stream synchronization; (2) stream layer, including synchronization across multiple streams either locally at a single receiver (i.e., inter-stream synchronization) or distributedly at multiple disparate receivers (i.e., group synchronization); (3) object layer, describing synchronization of both continuous and discrete media objects; and (4) specification layer, prescribing applications and tools for synchronization specifications.

Ehley at al's Model [24]. Ehley, Furth and Ilyas classified the synchronization technologies in 1994 based upon the synchronization locations, i.e., the places where the synchronization control schemes were performed (e.g., the capturing, distribution and presentation tiers in teleimmersion). The authors only investigated the inter-stream synchronization in their studies though.

Note that the above synchronization classification models are, in nature, either aligned with each other or mutually orthogonal. None of the models is able to describe the multi-modal multi-device setting of next-generation multi-site multi-functional interactive systems, with a support of the multi-tier pipeline configuration. A multi-dimensional synchronization model is needed to describe current modern multimedia technologies.

\section{- Synchronization Specification}

A further understanding of the multimedia synchronization topics required a more systematic specification methods to describe the synchronization problems. This promoted a number of specification models 
in these five years, which could generally be grouped into three categories (Fig. 2.3), according to Steinmetz $[9,18]$. In this dissertation, we focus on their impacts on the continuous multimedia systems like digital audio and video applications.

Axis-based specification. First proposed by Hodges, Sasnett and Ackerman in 1989 [57], the axisbased specification method aligns both continuous and discrete multimedia objects along either a real or virtual global timeline axis, based on the start and finish time of each object. The concept of a global time accessibility is owed largely by the invention of NTP in 1985 and its wide deployment since then. The duration of each media object must be described in the specification. For example, we can specify that a video clip is aligned with the duration between the 20-th and the 30-th seconds, while another audio clip is aligned with the duration between the 15-th to the 25-th seconds. This specification category is most widely employed in continuous multimedia applications.

Control-based specification. Developed by Steinmetz in 1990 [58], multimedia data are synchronized over a set of connected reference points, or synchronization points, based on which multimedia systems can detect synchronization errors and realign the continuous multimedia data. Oftentimes, these synchronization points can be placed periodically in order to allow consistent media resynchronization.

Interval-based specification. This specification method $[59,60,61]$ presents the logical temporal relations between two interval-based media objects without telling the exact start and finish time of each media object. For example, we can only specify if a video frame is presented before another video frame, but we are unable to tell the idle interval between two frames. This creates difficulty in describing the exact time of the continuous multimedia data.

To sum up, both axis-based and control-based specifications can serve the continuous multimedia synchronization in our study. We will extensively use the axis method with the global timeline support in this dissertation. We will also propose our own decision policies of synchronization points to characterize the multi-modal multi-stream configurations of next-generation interactive multimedia systems.

\section{- Synchronization Perception}

As people noticed more and more audio-visual synchronization skews on VOD and conferencing systems over the Internet, researchers became interested in understanding how large an audio-visual skew can be noticed by humans. Steinmetz and Engler conducted a subjective evaluation in 1993 [20, 62]. They recommended an in-sync region of a maximum 80-ms lip skew. They also showed that an out-of-sync skew of more than 160 ms was unacceptable.

The skews between multiple acoustic streams within a microphone array were also studied by Danner- 
berg and Stern in 1993 [63]. The authors claimed that a skew of $17 \mathrm{~ms}$ between the stereo audio signals could be perceivable, and that a maximum skew of $11 \mathrm{~ms}$ is preferable.

\section{- Intra-stream and Inter-stream Synchronization Control}

Researchers began to investigate the control framework in the early 1990s, exclusively for intra-stream and inter-stream synchronization of the video conferencing or on-demand systems, due to the rapid commercialization of these IP-based applications. Most studies in those early years focused on the synchronization of a single audio and a single video stream, where the audio stream was always selected as the reference stream with a master (audio) - slave (video) synchronization prototype, mainly due to the fact that the human perception was more sensitive to the degradations of audio signals. A global time was also assumed available between the audio and video signals by accessing NTP, even though different media could be captured at different devices.

In our study, we group different studies based on both location and functionality of synchronization control mechanisms. For synchronization locations, we investigate control algorithms at both sender and receiver sides. In terms of functionalities, we classify synchronization approaches that can either universally be shared by any media modality, or uniquely be applied to one specific modality. To facilitate the organization of our survey, we have also covered multiple control studies published after 1994.

For receiver-based synchronization, the buffering compensation has always been the most common approach to accommodate the intra-stream jitter and to minimize the inter-stream one-way delay heterogeneity. However, the abrupt adaptation of the buffer size can introduce the discontinuity of media presentation. To address this issue during the buffer size transition period, most studies perform the following methods $[23,33,64,65,66,67,68,69]$ :

To increase the buffering latency of a sensory stream:

1. Shared approach: (1) replicating past media frames; (2) interpolating media information by bidirectional data prediction based on nearby media data.

2. Video only: increasing the inter-frame period.

3. Audio only: (1) time scale modification without the pitch change; (2) inserting the stateless silence packets during the silence period.

On the other hand, to decrease the buffering latency of a sensory stream: 
1. Shared approach: skipping presentation of media frames.

2. Video only: decreasing the inter-frame period.

3. Audio only: (1) time scale modification without the pitch change; (2) reducing the stateless silence packets during the silence period.

For sender-based synchronization, the network bandwidth estimation and the resulting media data management are the two key components. The reason is that an insufficient bandwidth can exert Internet congestion jitter and losses which can affect both intra-media and inter-media synchronization. The bandwidth estimation can be achieved either by packet pair probing [70], or by monitoring the receiver jitter and loss statistics via the feedback control loop [71]. Based on the estimated bandwidth, the sender then perform multiple options of data management schemes [33, 66, 67, 72, 73, 74] which can include: (1) reducing the media sampling rate; (2) downgrading the media encoded quality; (3) skipping media data of lower priority; and (4) discarding media frames that cannot meet the receiver presentation deadline.

As discussed in Boronat at al's survey [19], which covers more than 100 synchronization papers), there have been numerous synchronization control studies in the multimedia history, where both sender- and receiver- based synchronization approaches are performed, either passively in response to Internet quality changes, or actively so as to prevent potential Internet degradations.

\subsubsection{Years of Blossoms: 1995-1999}

The multimedia synchronization continued to be a heated topic due to the revolutionary change of Internet quality and more sophisticated multimedia technologies. The support for massive multi-party involvement in various multimedia applications have intrigued the investigations of the (inter-receiver) group synchronization problems.

\section{- Historical Background}

The accessibility of broadband Internet became popularized in the late 1990s. This fostered the blossoms of multiple real-time applications, including the world's first commercial VoIP service by VocalTec in 1995 [75], the first 3D MMOG (called Meridian 59) by 3DO Company in 1995 [76], and the Caltech-CERN project in 1997 which built a virtual room videoconferencing system that was able to connect the research centers over the world [77]. 


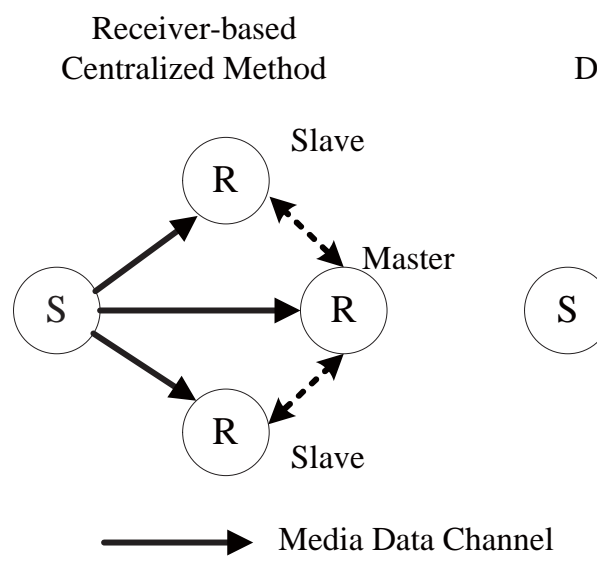

Receiver-based

Distributed Method

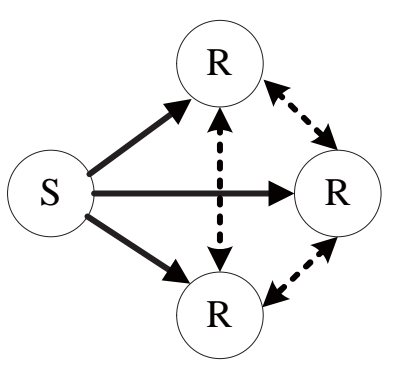

$4 \ldots \ldots \ldots \rightarrow$ Sync Control Channel
Sender-based

Method

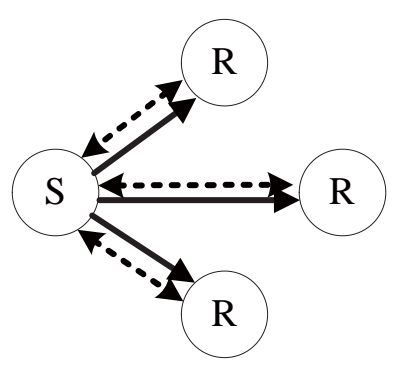

Figure 2.4: Group synchronization control: sender-based and receiver-based synchronization. S: sender site, R: receiver site.

In parallel with the development of new multimedia applications, the year of 1996 gave birth to lots of well-known ITU standards on multimedia codec specification and streaming protocols, including ITUT H.263 [78] on the video codec employed for communications with reduced bandwidth demand, and ITU-T H.323 [79] on packet-based multimedia communications systems. The IETF, on the other hand, established RFC 1889 [80], the Real-time Transport Protocol (RTP) specifying the standardized packet format for delivering streaming media over the Internet, and the RTP Control Protocol (RTCP), defining the control information for RTP data. Both ITU H.263, H.323 and IETF RFC 1889 experienced several iterations of updates and revisions since then. Later in 1999, the Moving Picture Experts Group established MPEG-4 as an ISO standard [81], describing the synchronization and multiplexing of compressed video and audio data.

The studies of intra-stream and inter-stream synchronization continued to prevail, due to more sophisticated multimedia applications and new multimedia standards. The evolution of multi-party conferencing systems and MMOG applications, owing to tremendously enhanced Internet bandwidth availability, had sparked the massive interests in realizing the group synchronization, for the purpose of preserving the fairness and the timing accordance among the players.

\section{- Group Synchronization Control}

Similar to intra-stream and inter-stream synchronization, the group synchronization control schemes can also be classified based on the synchronization locations and the functionalities of synchronization mechanisms.

1. Receiver-based synchronization (Fig. 2.4). One or multiple receivers need to decide the buffering time 
without the information from the sender sites. Based on the mechanisms of the synchronization controls, the receiver-based approaches can be further divided into two categories:

- Centralized (master-slave) method. In this method, one master receiver is selected as the synchronization reference site, and all receiver sites are the slave sites. The master receiver decides the buffer size of all slave sites within the multimedia sessions, based on their periodic feedback messages of current buffer statuses and one-way delay statistics between the sender and the sender sites [82, 83]. Note that in previous studies of 1990s and early 2000s, usually the master site is either ambiguously prescribed or is arbitrarily assigned, without consulting the real application functionalities of the studied multimedia systems, due in part to a lack of systematic modeling of heterogeneous human activities in these applications. While it is simple to implement the centralized method in the real multimedia systems, there are multiple serious drawbacks that may hinder its efficient operation. First, the connectivity between the master and slave receivers cannot be guaranteed due to the potentially poor Internet conditions and the firewall blocking issues. Second, the frequent message exchanges between the master and slave sites can exert heavy communication overhead that demands expensive bandwidth resources. Third, a timely synchronization adaptation in response to sudden Internet changes is unable to be achieved, because of the bidirectional latency between the master and slave sites. Fourth, the scalability is a common issue in the centralized method, where the computation resource is bottlenecked at the central master node. Fifth, receiver sites can easily join and leave in the multimedia applications like MMOG. When the master site no longer exists in the current session, the group synchronization of all other slave sites cannot be realized.

- Distributed method. In this method, each receiver site decides its own buffer size in a distributed fashion, by receiving periodic buffer status messages from all other receiver sites [84]. Compared to the centralized method, the bandwidth overhead due to the full-mesh message communication is tremendous. In addition, because each site performs synchronization adaptations without a collaboration, there can be serious mismatches and even conflicts in terms of the resulting synchronization performances at different receivers. These drawbacks prevent the adoption of the distributed method in real continuous multimedia applications.

2. Sender-based synchronization (or maestro synchronization) (Fig. 2.4). The sender site decides the buffer size of all receiver sites, based on their feedback messages [85]. Because synchronization control information are usually included in the media packet header sent from the sender to each receiver site, the 
resulting communication overhead can be effectively minimized, compared to the centralized receiver-based method. In addition, the reliability is no longer a problem when receiver sites are joining and leaving the multimedia session, as long as the sender site is consistently sending media data to the receivers. The sender-based synchronization is, by far, the best method to realize the group synchronization in the real systems, due to its flexibility, reliability and the implementation easiness. But the scalability and the timely synchronization adaptation are still two unsolved issues during the use of this method.

3. Multicast routing with bounded delay and delay variation. The design of multicast overlay with QoS support also became a heated topic in the late 1990s for multi-party multimedia applications (usually with a scale of no more than 10-15 end users). The (inter-receiver) group synchronization takes effect during the media distribution, by imposing the constraints of delay or/and delay variation over the multicast topology $[28,30,86,87]$. Note that a multicast overlay solely with delay constraint support can also lead to a bounded delay variation, in the sense that the delay variation cannot exceed the delay upper bound.

\subsubsection{Years of Maturity: 2000-2006}

The maturity of broadband Internet and multimedia technologies promoted the prosperity of thousands of IP-based interactive multimedia applications, which had become a part of everyday life. The popularity of mobile phones and laptops with wireless support allowed end users to access these applications on-thego, but the unreliable wireless quality (compared to the wireline connection) could affect the multimedia synchronization. The new century saw a need for additional sensory devices of new multimedia modalities and their broad usage in future systems. The researchers started to evaluate the roles of these cutting-edge technologies in the synchronization studies.

\section{- Historical Background}

The technological advancements in the early 2000s were mainly featured by three characteristics.

Scalability. To address the scalability issue of traditional server-client or multicast systems, peer-to-peer technologies were extensively deployed in the multimedia applications, e.g., the prototypes of peer-to-peer massively multiplayer gaming [88, 89], and the peer-to-peer TV system CoolStreaming [90].

Mobility. People were no longer satisfied with the standstill computing machines. With the invention of cellular GSM/3G technologies and IEEE 802.11 standards [91] for wireless communications, end users were allowed to connect their cell phones and laptops to the Internet anywhere that had wireless signals.

Diversity. The wide acceptance of haptics, accelerometer, body sensor and many other devices in a 
variety of multimedia systems offered users a fresh new experience. New applications in the early 2000s could be seen in haptic desktop [92], interactive haptic painting [93], wireless body sensory network for health monitoring [94] and accelerometer-based motion analysis systems [95].

The new multimedia configurations and applications introduced additional synchronization problems, thus, leading to plenty of fruitful synchronization studies.

\section{- Peer-to-peer Synchronization}

The delivery of live video (e.g., a live news program) via the peer-to-peer overlays requires the (interreceiver) group synchronization. This allows users to watch the same live videos in pace. The synchronization is only required at a coarse granularity, i.e., the inter-receiver skew is preferably within 10-30 seconds. The skew value is tolerable because end users are passively watching the videos without in-person participations of real activities.

The peer-to-peer synchronization is usually performed by constraining the delays (or the number of peer layers) over the video distribution tree/multi-tree topologies [96, 97, 98]. The achievable upper bound for the inter-receiver skews depends on the number of peer nodes and their bandwidth availability in the overlay.

\section{- Wireless/Mobile Synchronization}

Multiple control schemes previously used for the wireline synchronization have been modified and extended to adapt to the unique characteristics of wireless/mobile networks.

1. Poor network quality. Generally, wirless/celluar connections are more prone to jitter and losses due to the uncontrollable interference signals using the same unlicensed spectrum [99], and the available bandwidth resources are also limited. In [100], the authors address the issue by minimizing the number of synchronization control packets, forecasting wireless link quality, based on which new sender resource allocation and receiver buffer management schemes are developed.

2. Handover. The handover (from one base station or access point to a different one) is another emerging issue in the wireless/mobile synchronization. In general, bursty network quality degradation can be incurred during the short handover period [101]. [102] has solved the problem by proposing a multi-phase handover management scheme in order to minimize the bursty impact.

3. Scheduling in base station/access point. The packet scheduling algorithms in the base stations or access points can affect the delay when media data are being streamed to the mobile clients. [103] has evaluated and compared four scheduling algorithms, and their resulting delay impacts. 


\section{- Synchronization Control of New Media}

Multiple studies [104, 105] have been done to investigate the reliability of intra-stream, inter-stream and group synchronization control schemes when new media modalities were being added to the multimedia applications. Their evaluations were performed by streaming real haptic packets over a controllable network. They showed that existing synchronization control schemes could work well with minimal modifications for the haptic data. But similar to previous studies, these work lacked an unambiguous methodology for selecting the synchronization references, when different multi-modal media information were employed to offer heterogeneous contributions to end user experience.

\subsubsection{Years of Leaps: On and After 2007}

Next-generation multimedia systems are becoming more powerful in terms of the accessibility of computation and network resources, more complex in terms of both hardware and software configurations, and more versatile in terms of the functionalities that can be performed. The leaps of numerous modern multimedia and networking technologies and their integration into a single application has led to many open synchronization problems that await researchers to delve into.

\section{- Historical Background}

The prosperity of multi-modal sensory devices has changed what traditional multimedia applications were supposed to look like. In addition to standard audio-visual I/O support, multimedia systems like telepresence [106] or teleimmersion are now configured, via either wireline or wireless, with multiple distributed multi-modal media sensors at the sender sites, and their corresponding rendering machines at the receivers. To perform multi-modal multi-stream synchronization, the sender site identifies the (global) captured timestamp of each media frame, and this time correlation can allow the media data to be presented at distributed receiver output devices at the same time.

The rapid leaps of cloud computing infrastructure has also offered a backend support for computationintensive multimedia signal processing in numerous applications. One example is the real-time cloud-based encoding of high-quality images, which has been deployed in many on-demand or conferencing applications on the market [107].

The maturity of stereoscopic cameras has advanced the popularity of 3DTV as well. Synchronization across the multiview images is critical due to their strict temporal correlation. [108] proposes a peer-to-peer overlay that can preserve this correlation during the 3D multiview video distribution. 


\section{- RTP/RTCP based Synchronization Control Implementation}

The RTP and RTCP protocols have been used extensively in implementing different synchronization control schemes $[109,110]$. Generally, the timestamp field in the RTP header can tell the time correlations of multi-modal media data within/across streams, and thus, enabling both intra-stream and inter-stream synchronization. The RTCP protocol, on the other hand, prescribes the specifications for communicating synchronization control update messages. In addition to RTP/RTCP, several other study groups have been working on two new RFC standards. Specifically, one is RFC 6051 [111] titled "Rapid Synchronization of RTP Flows", aimed at reducing the initial synchronization delay for both unicast and multicast scenarios. The other [112] (still in the draft) is titled "RTCP for inter-destination media synchronization", which, as its name suggests, extends existing RTCP control specifications to provide necessary underlying protocol support for the (inter-receiver) group synchronization.

However, neither RTP nor RTCP is able to specify the synchronization references for each synchronization demand, which can become complicated under the scalability of multi-modal multi-device configurations (Section 3). In addition, neither of the protocols has the ability to coordinate multiple distributed output devices at a same receiver site, in order to achieve their in-sync multi-modal media presentation. This leads to the emergence of the multi-device synchronization problem.

\section{- Multi-device Synchronization and Precision Time Protocol (PTP)}

To allow synchronous multimedia presentation at distributed rendering devices, a multi-device synchronization scheme is in urgent demand $[113,114,115,116]$. Generally, it adopts a centralized control policy which requires two components, as shown in Fig. 2.5:

Synchronization module. The synchronization module decides the in-sync multimedia presentation time, and informs the device modules by periodic control messages. The synchronization module can be implemented either on one of the device machines (Option 1 in Fig. 2.5), or on another specialized computer that has active communication channels with all the end devices (e.g., the receiver gateway in our teleimmersive testbed, as shown in Option 2 of Fig. 2.5).

Device module. The device modules, located on different end device machines, compute the buffering delays of media data based on the received control messages from the synchronization module. Each device module then schedules their presentation according to the local clock. Note that this mechanism demands the clock synchronization across the device machines, which can be achieved either by NTP, or by sending round trip packets to estimate the inter-device clock skews. However, both approaches can only offer a 


\section{Option 1}

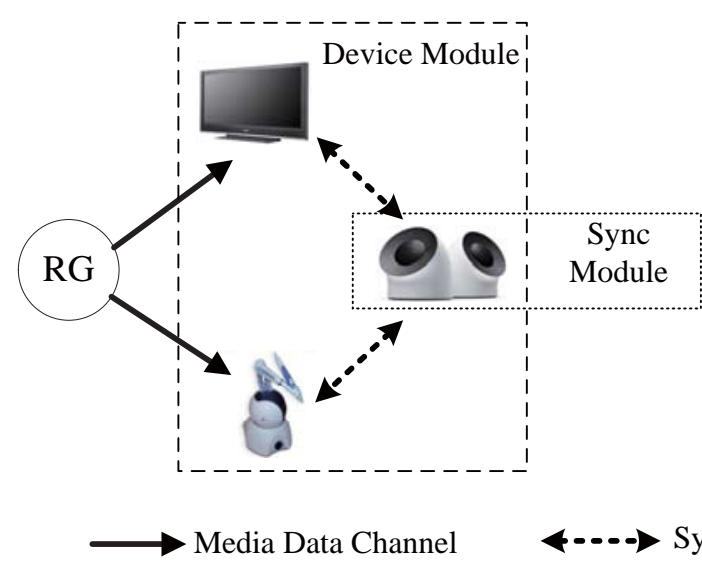

Option 2

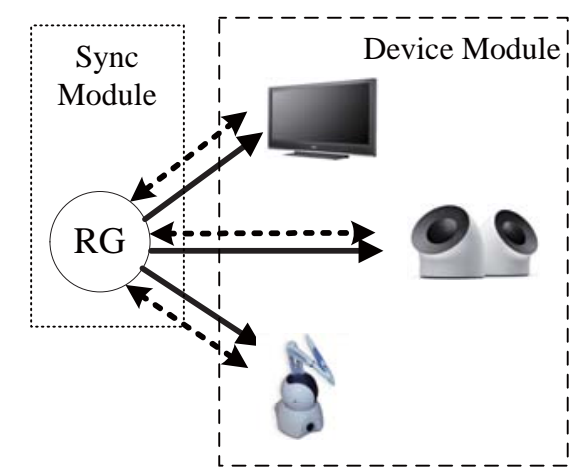

RG Receiver Gateway

Figure 2.5: Multi-device synchronization. Option 1: synchronization module is one of the device machine; Option 2: synchronization model is the specialized machine (i.e., receiver gateway).

synchronization accuracy of $10 \mathrm{~ms}$.

In case of a need for a finer granularity, the IEEE 1588 Precision Time Protocol (PTP) [117] can be employed. PTP is able to achieve a clock accuracy up to the range of sub-microseconds in the local area network, where the rendering devices are usually located. PTP can perform better clock synchronization than NTP, because it is able to quantify the clock quality by estimating its timing deviation, and to monitor the stability of the clocks by consulting the PTP reference.

\section{- Synchronization Perception of New Media}

There are also a number of subjective studies investigating the synchronization perceptual impacts in different modern multimedia applications.

[21] evaluates the synchronization in the mobile terminal with a maximum of Quarter Common Intermediate Format (QCIF) image size 176x144. It shows that in the mobile setting with a video frame rate below $15 \mathrm{fps}$, people are more tolerant to a synchronization error when the video spatial resolution is reduced. It also exhibits that the annoying threshold of a lip skew can be large as 200-300 ms due to the degraded motion smoothness.

[118] evaluates the subjective quality of the skew between the haptics and the video data. The paper shows that a skew below $40-80 \mathrm{~ms}$ is hardly perceptible, and that the skew greater than $300 \mathrm{~ms}$ is annoying.

[119] conducts a perceptual measurement of the impact of synchronization skews between the olfactory data and audio-visual content, assuming the audio-visual lip skew is zero. Their results show a synchro- 
Table 2.1: Absolute Category Rating.

\begin{tabular}{|c|c|}
\hline Score & Explanation \\
\hline \hline 5 & Excellent \\
4 & Good \\
3 & Fair \\
2 & Poor \\
1 & Bad \\
\hline
\end{tabular}

nization threshold of $-30 \mathrm{~s}$ when olfaction is ahead of audiovisual data, and of $+20 \mathrm{~s}$ when olfaction is behind. The paper also evaluates the skew impact on the acceptability of the olfactory media. Participants are asked if "the olfactory smell was distracting" or "annoying" when the synchronization skews between olfactory and audio-visual data are introduced for different video clips. The results demonstrate that a mis-synchronization has minimal impact on the olfactory perception.

[120] also measures the quality of olfactory-haptic skew. The authors present that the annoying threshold is in the range of 1-3 s.

In terms of the (intra-media) synchronization quality of the 3D stereoscopic videos, [121] conducts a comprehensive subjective test on four scenes, and argues that a temporal asynchrony of $120 \mathrm{~ms}$ between the left and right views is satisfactory, and an asynchrony of $280 \mathrm{~ms}$ can lead to a very poor synchronization perception. The authors also show that the asynchrony detection threshold can be different depending on the heterogeneous scenery contents.

\subsection{Subjective Rating Method and Subjective Metrics}

The human perception is another factor guiding the synchronization control adaptations. In evaluating the perceptual impacts, multiple subjective rating methods and subjective metrics have been proposed and standardized in the past.

\subsubsection{Subjective Rating Method}

\section{- Absolute Category Rating (ACR)}

ITU-T BT.500 has proposed the absolute category rating (ACR) method, in which participants observe one single media sample (e.g., a video sequence) and give an ACR score from 1 to 5 (a higher score is better), as presented in Table 2.1 . 
Table 2.2: Degradation Category Rating.

\begin{tabular}{|c|c|}
\hline Score & Explanation \\
\hline \hline 5 & Imperceptible \\
4 & Perceptible, but not annoying \\
3 & Slightly annoying \\
2 & Annoying \\
1 & Very annoying \\
\hline
\end{tabular}

While number of paper $[122,123,124,125]$ have employed ACR in their studies, the problem of the rating method, however, is that a standard rating scale is missing due to the absence of a reference sample (i.e., a prescribed sample with the best possible quality). Hence, the participants in the ACR studies usually give a score based on their own expertise. This leads to the non-uniform distributions of rating scores, which can invalidate the subjective results.

\section{- Degradation Category Rating (DCR)}

To address the ACR drawback, ITU-T P.910 [35] proposes an alternative assessment method called the degradation category rating (DCR), in which participants now observe two media samples. The first media sample of the pair is always the source reference with the best-possible quality, while the second one is the same source but degraded by various test settings. Each participant must give a DCR score ranging from 1 to 5 (a higher score is better), as shown in Table 2.2.

Some representative work using DCR are $[22,126,127,128]$. Note that the first media sample (i.e., the one with the best-possible quality) may not always be played during the DCR test.

\section{- Comparative Category Rating (CCR)}

A third rating method proposed by ITU-T P.910 is the comparative category rating (CCR), where each participant in the user study is required to watch and compare two media samples of the same source, both degraded by different test conditions, and to give a vote ranging from -3 to 3 . A negative/positive score means the first sample is worse/better than the second sample, and its absolute value represents the quality difference level of the two samples. The details of CCR scores are presented in Table 2.3. Existing studies employing CCR to conduct pairwise comparisons include [129, 130].

\section{- Other Rating Methods}

The above three subjective rating methods have shown multiple variations in different forms. For ex- 
Table 2.3: Comparative Category Rating.

\begin{tabular}{|c|c|}
\hline Score & Explanation \\
\hline \hline 3 & Much better \\
2 & Better \\
1 & Slightly better \\
0 & About the same \\
-1 & Slightly worse \\
-2 & worse \\
-3 & Much worse \\
\hline
\end{tabular}

Table 2.4: An example of user votes.

\begin{tabular}{|c|c|c|c|c|c|c|c|c|c|}
\hline \multirow{2}{*}{ Case } & \multicolumn{2}{|c|}{ Media Sample } & \multicolumn{6}{|c|}{ \#User votes for each score } \\
\cline { 2 - 10 } & Sample 1 & Sample 2 & Score -3 & Score -2 & Score -1 & Score 0 & Score 1 & Score 2 & Score 3 \\
\hline \hline 1 & A & B & 0 & 0 & 0 & 1 & 12 & 6 & 0 \\
\hline 2 & C & D & 0 & 1 & 8 & 1 & 8 & 1 & 0 \\
\hline
\end{tabular}

ample, Sat and Wah [40] shrink the CCR scales to only 3-level score set: $-1,0$, and 1, indicating the first media sample is worse/same/better than the second one. On the other hand, Chen et al [131] proposes the OneClick rating framework by reducing the ACR scale range to only two scores: 1 and 0 . Participants in their framework are asked to evaluate if a media sample is satisfactory or not. If it is satisfactory, participants need to click on a specialized button on the screen indicating a score 1. Otherwise the media sample is rated as score 0. Steinmeitz [20] asks users to evaluate the impairment of a media sample in three scales: unnoticeable, noticeable, and annoying. His methodology can also be classified as the ACR category. ITU-R BT.1438 [132] has been proposed to assess the stereoscopic television pictures exclusively, and this standard follows the similar methods as DCR with a scale ranging from 0 to 100 .

\subsubsection{Subjective Metrics}

Many subjective metrics have been proposed in the past to analyze and present the resulting user votes of above ACR, DCR, CCR. We will use an example to better explain these metrics.

\section{- Example}

We suppose 19 people are participating in the user study. They are asked to perform two pairwise comparisons using CCR. The first comparison is between media samples A and B, and second is between media samples $\mathrm{C}$ and D. The results of user votes are presented in Table 2.4. 


\section{- Mean-Opinion-Score}

As discussed in Section 1.5.3, ITU-T BT.500 and P.910 have proposed three subjective metrics to capture the average value of the user votes in ACR, DCR and CCR tests respectively: the mean-opinion-score (MOS), the degradation mean-opinion-score (DMOS) and the comparative mean-opinion-score (CMOS). Mathematically, if we suppose $\left\{X_{1}, X_{2}, \ldots, X_{n}\right\}$ is list of $n$ user votes, the average $\bar{X}$ (i.e., MOS-like metrics) can be computed as:

$$
\bar{X}=\left(X_{1}+X_{2}+\cdots+X_{n}\right) / n
$$

For example, from Table 2.4, we can conclude that CMOS of the first case is 1.26 , while CMOS of the second comparison is 0 .

Existing studies $[22,122,123,124,125,126,129]$ have been heavily using these subjective metrics by assuming a Gaussian distribution of the resulting votes, which is generally true in ACR and DCR tests. But when the variance of the votes is large and a Gaussian model does not follow, the resulting average value can lack statistical significance. The issue becomes highlighted in interactive multimedia systems, where the overall human perception is dominated by multiple quality attribute dimensions. The tradeoffs among these dimensions in a CCR comparison test can trigger the diversity of human voting preferences, because each participant may pay different attentions to heterogeneous quality dimensions. This will lead to a potential output of contradicting votes, where participants do not agree on which one is better at a comparison of two media samples. Some participants may give a positive comparison score, while others can output a negative score. This phenomenon has been proven in our past VoIP comparison studies [36, 40, 133], where one media sample is with better interactivity but a worse media signal intelligibility, and the other sample is with better media intelligibility but a worse interactivity. The second case in Table 2.4 demonstrates an example, which leads to a CMOS score of 0.

\section{- Confidence Interval}

To address the drawback of the MOS-like metrics, several other subjective metrics have been investigated. One metric commonly used to describe the variance of user votes and the statistical significance of MOS-like metrics is the confidence interval (CI). In evaluating the subjective quality of multimedia systems, a $95 \% \mathrm{CI}$ is often calculated as:

$$
\mathrm{CI}=\left(\bar{X}-1.96 \frac{\sigma}{\sqrt{n}}, \bar{X}+1.96 \frac{\sigma}{\sqrt{n}}\right)
$$

In the above equation, $\bar{X}$ can be obtained from Eqn 2.2, and $\sigma$ is the sample variance which is defined as: 


$$
\sigma=\frac{1}{n-1} \sum_{i=1}^{n}\left(X_{i}-\bar{X}\right)^{2}
$$

For example, in Table 2.4, case 1 leads to a CI of $(1.12,1.41)$, while case 2 has a a much larger CI of $(-0.600 .60)$. A larger interval size means a greater diversity of user votes.

While CI is a step forward to show the user voting diversity, the metric is still inherently based on the Gaussian assumption of the votes. It is unable to explicitly tell which opinion has the majority votes, or whether the quality of a media sample is better than or equal to that of another sample. This issue may lead to a vague conclusion in analyzing the CCR results.

\section{- Distribution of User Votes}

Another subjective metric aims to classify the user votes in multiple categories, compute the number of votes in each category, and represent the perceptual results using the category-based voting distribution. For example, in CCR, we may represent the distribution in either a triplet or a duplet value.

Triplet Metric. The first method is to use a triplet to describe the distribution: $\left(n_{<0}, n_{=0}, n_{>0}\right)$ representing the number of votes that is less than, equal to, or greater than 0 (i.e., the first media sample is worse than, equal to, better than the second sample). Given that $n$ is the number of total votes, the following equation satisfies:

$$
n_{<0}+n_{=0}+n_{>0}=n
$$

For example, we can represent the first case in Table 2.4 as $(0,1,18)$, and the second case as $(9,1,9)$.

Duplet Metric. The second method [129] is to present the distribution of CCR votes in duplet $\left(n_{\leq 0}, n_{\geq 0}\right)$, where $\left(n_{\leq 0}\right.$ is the number of votes who think the first media sample is no better than second one, and $\left(n_{\underline{ }}\right.$, the first media sample is no worse than second one. The duplet value can be computed as:

$$
\begin{aligned}
& n_{\leq 0}=n_{<0}+n_{=0} / 2 \\
& n_{\geq 0}=n_{>0}+n_{=0} / 2
\end{aligned}
$$

Note that $n_{=0}$ can be odd, and the resulting ( $n_{\leq 0}$ and $\left.n_{\geq 0}\right)$ may no longer be integers. [129] addresses this issue by doubling the original duplet value. For example, according to [129], the first case in Table 2.4 can be represented as $(1,37)$, while the second case as $(19,19)$.

The major problem of this duplex metric is that it removes the option that the qualities of two media samples can be qual. Because the "equal" opinion is very common in interactive multimedia systems, we will adopt the triplet metric in our study. 


\section{- Dominant Opinion}

This metric is used exclusively for the CCR method. Oftentimes, we are unable to derive from the triplet metric $\left(n_{<0}, n_{=0}, n_{>0}\right)$ and tell with confidence which is the dominant opinion, the opinion that the majority (say, more than 50\%) of votes will agree on, whether it is the opinion that one media sample is better/worse than the other, or that the qualities of two samples are about the same. The case 2 of Table 2.4 shows a good example where it is unable to locate a dominant opinion at $\left(n_{<0}, n_{=0}, n_{>0}\right)=(9,1,9)$. Our past study [37] uses the hypothesis test to address this issue.

In [37], we first compute the voting probabilities as:

$$
\left(p_{<0}^{s}, p_{=0}^{s}, p_{>0}^{s}\right)=\left(\frac{n_{<0}}{n}, \frac{n_{=0}}{n}, \frac{n_{>0}}{n}\right)
$$

In order to determine the dominant opinion from $\left(p_{<0}^{s}, p_{=0}^{s}, p_{>0}^{s}\right)$ with $p \geq 50 \%$ probability and a certain level of statistical significance, we model the subjective dominant opinions (among $\{<0,=$ $0,>0\}$ corresponding to the three options \{ "worse", "same", "better"\}), by a multinomial distribution with 3 possible outcomes, assuming the independence of subjects. We then conduct the hypothesis test by selectively combining two options within the 3 outcomes, and having an equivalent binomial distribution that represents the for or against probabilities of the opinion.

An option $i$ ( $i$ can be one of the three options: $<0,=0,>0)$ is dominant if the following hypothesis is accepted:

$$
H_{0}:\left(p_{i}^{s}, \sum_{j \neq i} p_{j}^{s}\right) \text { is drawn from binomial }(n, p \geq 0.5)
$$

where $H_{0}$ is the null hypothesis. Given the significant level $\alpha$ (the significance value that the tests can rule out the null hypothesis) and P-value (the probability of votes for the dominant opinion from binomial $(n, p \geq$ $0.5)$ ), we are able to find $n_{\min }$, the minimal number of subjects out of $n$ that needs to agree on a dominant opinion:

$$
n_{\min }=\arg \min _{k} \sum_{i=0}^{k}\left(\begin{array}{c}
n \\
i
\end{array}\right) \cdot 0.5^{i} \cdot 0.5^{n-i} \geq 1-\alpha
$$

[37] prescribes that the comparison of two media samples is "inconclusive" if there is no dominant opinion in $\left(p_{<0}^{s}, p_{=0}^{s}, p_{>0}^{s}\right)$ (i.e., $n_{<0}, n_{=0}, n_{>0}$ are all less than $\left.n_{\min }\right)$.

For example, for $90 \%$ significance ( $\alpha=10 \%$ ), we know from Eqn. 2.10 that at least 13 out of 19 votes (i.e., at least $68 \%$ of votes) need to agree on a dominant opinion. Hence, the dominant opinion in case 1 of Table 2.4 is that the first media sample is "worse" than the second media sample. Case 2 leads to an 
"inconclusive" comparison.

We have shown in [37] that the inconclusive comparison is very likely in interactive multimedia systems, where the tradeoffs of multi-dimensional quality attributes become prevalent. But the scope of [37] is limited to VoIP systems, where we have only considered the tradeoff between the audio signal intelligibility and the interactive quality.

\subsection{Human Perception in Multimedia Applications}

Existing researched studies and industrial standards have investigated human perceptual quality of multimedia applications based on the rating methods and subjective metrics that we have discussed. But since these rating methods and metrics have their own applications and limitations, their choices depend on the nature and purpose of the experiments. In this section, we will survey the existing studies and standards, and will present the results that are relevant to the synchronization quality, interactivity and media signal intelligibility in interactive multimedia.

\subsubsection{Survey Description}

Based on the methodology of the subjective evaluations, past studies on multimedia perception can generally be divided into three categories:

1. Studies investigating the impact of a single quality dimension, by assuming all other quality dimensions as optimal (best-possible) [20, 136, 147]).

2. Studies focusing on multiple quality dimensions, but without investigating their cross/combined impacts $[131,153]$.

3. Studies working on the cross/combined effects of multiple quality dimensions $[22,37,40,126,133]$.

Table 2.5 presents a survey of existing studies, along with their applications, studied media and quality dimensions, and their subjective rating methods. The type of media applications can be teleimmersion, traditional video/audio conferencing or on-demand videos. The studied media can be either video (2D or 3D), or audio. The identified quality dimensions are the video signal intelligibility (including the frame rate, the spatial resolution, the encoded quality and other 3D-specific factors), the audio signal intelligibility, the interactive quality (either one-way delay [22, 126, 153] or VoIP conversational response delay [40, 
Table 2.5: A survey of subjective quality assessment. The types of studied multimedia systems include audio and 2D video conferencing (CONF), 2D video-on-demand (VOD), 3D stereoscopic video-on-demand (STEREO) and teleimmersive (TI) applications. VSI: video signal intelligibility, which includes the video frame rate (FR), the spatial resolution (RES), the encoding quality (ENC) and the 3D-specific factors (3D). ASI: audio signal intelligibility. Three subjective rating methods are classified: ACR, CCR and DCR. Y: representing the corresponding quality dimension is studied.

\begin{tabular}{|c|c|c|c|c|c|c|c|c|c|}
\hline & \multirow{2}{*}{ Type } & \multicolumn{2}{|c|}{ Studied Media } & \multirow{2}{*}{ VSI } & \multirow{2}{*}{ ASI } & \multirow{2}{*}{ Interactivity } & \multirow{2}{*}{ Sync } & \multirow{2}{*}{ Method } & \multirow{2}{*}{ Comments } \\
\hline & & Video & Audio & & & & & & \\
\hline $\bar{c}[22]$ & CONF & $2 \mathrm{D}$ & $\overline{\mathrm{Y}}$ & FR, RES, ENC & $\overline{\mathrm{Y}}$ & One-way delay & $\bar{Y}$ & $\overline{\mathrm{DCR}}$ & Dependent \\
\hline [126] & CONF & & $\mathrm{Y}$ & & $\mathrm{Y}$ & One-way delay & & DCR & Dependent \\
\hline [133] & CONF & & $\mathrm{Y}$ & & $\mathrm{Y}$ & Response delay & & $\mathrm{CCR}$ & Dependent \\
\hline [37] & CONF & & $\mathrm{Y}$ & & $\mathrm{Y}$ & Response delay & & $\mathrm{CCR}$ & Dependent \\
\hline [40] & $\mathrm{CONF}$ & & $\mathrm{Y}$ & & $\mathrm{Y}$ & Response delay & & $\mathrm{CCR}$ & Dependent \\
\hline [134] & VOD & $2 \mathrm{D}$ & & FR & & & & $\mathrm{ACR}$ & \\
\hline [20] & VOD & 2D & $\mathrm{Y}$ & & & & $\mathrm{Y}$ & ACR & \\
\hline [21] & VOD & $2 \mathrm{D}$ & $\mathrm{Y}$ & FR & & & $\mathrm{Y}$ & $\mathrm{ACR}$ & Dependent \\
\hline [129] & VOD & 2D & & FR, RES, ENC & & & & $\mathrm{CCR}$ & Dependent \\
\hline [135] & VOD & 2D & & ENC & & & & DCR & \\
\hline [136] & VOD & 2D & & ENC & & & & ACR/DCR & \\
\hline [127] & VOD & 2D & & FR & & & & DCR & \\
\hline [128] & VOD & 2D & & FR & & & & DCR & \\
\hline [137] & VOD & 2D & & FR, ENC & & & & $\mathrm{ACR}$ & Independent \\
\hline [138] & CONF & $2 \mathrm{D}$ & & FR, RES & & & & ACR & Dependent \\
\hline [139] & VOD & $2 \mathrm{D}$ & & FR, RES, ENC & & & & $\mathrm{ACR}$ & Dependent \\
\hline [140] & VOD & 2D & & FR & & & & $\mathrm{ACR}$ & \\
\hline [141] & VOD & 2D & & FR & & & & $\mathrm{ACR}$ & \\
\hline [142] & CONF/VOD & $2 \mathrm{D}$ & $\mathrm{Y}$ & ENC & $\mathrm{Y}$ & & & DCR & Dependent \\
\hline [124] & VOD & $2 \mathrm{D}$ & $\mathrm{Y}$ & FR & & & $\mathrm{Y}$ & $\mathrm{ACR}$ & Dependent \\
\hline [122] & VOD & $2 \mathrm{D}$ & $\mathrm{Y}$ & FR & $\mathrm{Y}$ & & & $\mathrm{ACR}$ & Dependent \\
\hline [123] & CONF & 2D & $\mathrm{Y}$ & FR & $\mathrm{Y}$ & & & $\mathrm{ACR}$ & Dependent \\
\hline [143] & $\mathrm{CONF}$ & 2D & $\mathrm{Y}$ & FR & $\mathrm{Y}$ & One-way delay & & $\mathrm{ACR}$ & Dependent \\
\hline [144] & $\mathrm{CONF}$ & 2D & $\mathrm{Y}$ & FR & $\mathrm{Y}$ & & & $\mathrm{ACR}$ & Dependent \\
\hline [145] & VOD & 2D & & & & & $\mathrm{Y}$ & $\mathrm{ACR}$ & \\
\hline [146] & VOD & 2D & $\mathrm{Y}$ & (FR, ENC) & $\mathrm{Y}$ & & & $\mathrm{ACR}$ & Dependent \\
\hline [147] & CONF & & $\mathrm{Y}$ & & & One-way delay & & $\mathrm{ACR}$ & \\
\hline [148] & $\mathrm{CONF}$ & & $\mathrm{Y}$ & & & One-way delay & & $\mathrm{ACR}$ & \\
\hline [149] & CONF & & $\mathrm{Y}$ & & & One-way delay & & $\mathrm{ACR}$ & \\
\hline [150] & CONF & & $\mathrm{Y}$ & & & One-way delay & & $\mathrm{ACR}$ & \\
\hline [151] & CONF & 2D & $\mathrm{Y}$ & & & & $\mathrm{Y}$ & $\mathrm{ACR}$ & \\
\hline [131] & CONF & & $\mathrm{Y}$ & & $\mathrm{Y}$ & One-way delay & & ACR & Independent \\
\hline [152] & CONF & & $\mathrm{Y}$ & & $\mathrm{Y}$ & One-way delay & & $\mathrm{ACR}$ & Independent \\
\hline [125] & TI & $3 \mathrm{D}$ & & $3 \mathrm{D}$ & & & & $\mathrm{ACR}$ & \\
\hline [153] & TI & $3 \mathrm{D}$ & & $3 \mathrm{D}$ & & One-way delay & & $\mathrm{ACR}$ & Independent \\
\hline [154] & TI & $3 \mathrm{D}$ & $\mathrm{Y}$ & $3 \mathrm{D}$ & $\mathrm{Y}$ & & & ACR & Independent \\
\hline [130] & TI & $3 \mathrm{D}$ & $\mathrm{Y}$ & 3D, FR, RES & $\mathrm{Y}$ & & & $\mathrm{CCR}$ & Independent \\
\hline [155] & STEREO & $3 \mathrm{D}$ & & $3 \mathrm{D}$ & & & & $\mathrm{ACR}$ & \\
\hline [156] & STEREO & $3 \mathrm{D}$ & & $3 \mathrm{D}$ & & & & $\mathrm{ACR}$ & \\
\hline [121] & STEREO & $3 \mathrm{D}$ & & $3 \mathrm{D}$ & & & $\mathrm{Y}$ & ACR & Dependent \\
\hline
\end{tabular}


$133,152]$ ), and the synchronization quality. The subjective rating methods can be ACR, CCR or DCR. The column of comments specifies whether the cross/combined effects (dependencies) of multiple studied quality dimensions are identified in the study, or each dimension is independently evaluated.

Among these assessments, perhaps ITU-T G.107 [126] and G.1070 [22] are those that are closest to our study, because both standards investigate the cross/combined effects of multiple quality dimensions similar to what we have identified in Section 1.4. But both standards are only for VoIP and 2D video conferencing. None of these assessments is able to describe the combined effect of multiview video macroframe rate, audio quality, interactive response delay and audio-visual synchronization skew in a teleimmersive setting. We will briefly discuss the perceptual impact of each quality dimension as follows.

\subsubsection{Media Signal Intelligibility}

\section{- Audio Intelligibility}

There has been a number of research studies on evaluating the audio signal intelligibility. [146], for example, finds a mapping between the audio data rate and the human perception in MOS, using the ACR method. $[131,152]$ have also conducted subjective studies evaluating the impact of Internet jitter on audio signal perception.

For industrial standards, ITU-T G.107 presents a subjective model based on the audio signal-to-noise ratio, the echo effect and other impairments that occur simultaneously with the voice signals (e.g., too loud speech level and the quantization noise). ITU-T P.862 [157] proposes the metric Perceptual Evaluation of Speech Quality (PESQ) which is already able to describe the real human subjective perception on audio signals based on a psycho-acoustic scale. PESQ has since been heavily employed in various other VoIP studies including $[40,133,158]$.

\section{- Video Intelligibility}

Many studies have also evaluated the video signals, under the impact of the video frame rate $[127,129$, $139,140,141]$, the spatial resolution $[129,139]$ and the encoded video quality $[135,136,139]$, respectively. Generally, the human perceptual quality can be improved at an increased video frame rate, a larger spatial resolution, and a better encoded quality. Specifically, in [134], the authors utilize an exponential model to identify the impact of 2D video frame rate on the video signal degradations. Since the teleimmersive 3D multiview videos will eventually render on a $2 \mathrm{D}$ display, this mathematical model can serve as a theoretical 
foundation for our study.

Recently, the research community has also investigated the human perception in watching 3D stereoscopic images $[155,156]$. Because these studies require a specialized 3DTV setup, their results cannot be directly applied to our work.

On the other hand, ITU-T G.1070 estimates the video signal quality based on the coding distortion and packet losses robustness. The standard focuses on the video image artifacts by assuming the availability of some loss concealment mechanisms within the $2 \mathrm{D}$ video codec. These metrics, however, are inapplicable to the current teleimmersive multiview videos.

\section{- Combined Audio-visual Intelligibility}

It has been proven in $[122,142]$ that the intelligibility of audio and video signals have cross impacts. [122], for example, argues that when people are evaluating the audio signal of an audiovisual stimulus, the video signals will have great impacts on the perceived audio intelligibility. The reverse effect, the effect of audio signals in judging the video intelligibility, can be negligible. The paper further shows that the video signals dominate the combined audio-visual signal perception in their nonconversational experiments.

\subsubsection{Synchronization Quality}

We have presented in Section 2.1 a list of works on the synchronization perception. But its relations with media signal intelligibility, interactive quality and video content heterogeneity have yet been systematically studied.

Many studies evaluate the media signal quality alone without considering the other factors. For example, the studies $[22,145,151]$ assume perfect media signal quality during the synchronization evaluations, and they do not take into account the video content factor.

On the other hand, [20] evaluates the lip synchronization under different contents: header, shoulder and body views. It shows that the three views lead to different perceptual impacts. But this difference is not further modeled in the paper. [21] demonstrates that the synchronization quality is affected by the video signal intelligibility, and that a lower video frame rate and smaller spatial resolution can increase the audio-visual detectable asynchrony threshold. But it does not manage to provide a form to describe the dependency among the quality attributes. 


\subsubsection{Interactivity}

Previous studies on the interactive quality (delay impairment) can be divided into two categories based on their applications. For packet-switched telephone network, Kiatawaki and Itoh [149] study the pure delay effect on speech quality, and their results show that one-way delays are detectable and can influence listeners' subjective assessment. Richards [147] and Brady [148] conclude from their subjective evaluations that longer delays can decrease the user satisfaction rate.

For Internet conference, ITU-T G.114 [150] prescribes that a one-way delay of less than $150 \mathrm{~ms}$ is desirable and a delay of more than $400 \mathrm{~ms}$ is unacceptable in a two-party VoIP. On the other hand, ITUT G.107 uses a complex sixth-order model to describe the VoIP delay impairment, while ITU-T G.1070 employs a linear function to present the delay impacts in the 2D video conference. G.1070 standard also shows that the delay degradation is much smaller than VoIP applications.

Unfortunately, none of these studies and standards take into account the impacts of other quality dimensions on the interactive quality. Our past studies on VoIP $[40,133]$ argue that such perceptual impacts do exist between the audio signal intelligibility and the interactive quality, and that the resulting combined human perceptual mapping cannot be described as a closed form by the two quality dimensions.

\subsubsection{Remarks}

The complex cross impacts among the media signal intelligibility, the interactive quality and the synchronization quality have created difficulties in assessing the combined human perceptual effects in a closed form. We have seen from the above survey that lots of existing studies choose to neglect this issue by simply investigating the impacts of individual quality dimensions, while many others present the multidimensional dependency findings without resorting to a systematic mathematical representation. None of them is able to tell explicitly the exact value of the employed subjective metrics under unseen conditions of the three quality dimensions. It is, of course, impossible to conduct subjective evaluations over all possible qualities, because subjective tests are expensive and time-consuming. Hence, in order to guide the online system controls and consistently offer the best human perceptual quality, offline generalization of the subjective results is required. This can usually be achieved by machine learning approaches. Our past studies [40, 133] employ support vector machine (SVM) [41] tool to realize the generalization. We will use the same method in this dissertation. 


\subsection{Summary}

In this chapter, we have presented a complete survey of related works on the multimedia synchronization and the human perceptual assessments. We have provided a historical view of different synchronization studies and standards that have been proposed since they were introduced in 1980s. We have also presented an all-around analysis of the perceptual assessment studies, with a focus on the classification methodology for existing subjective user studies, and the pros and cons of current subjective metrics. Understanding all these related works will help better design a synchronization control framework for high-quality multi-site teleimmersion.

\subsection{Table of Abbreviations and Notations}

\section{List of Abbreviations in Chapter 2:}

$\begin{array}{ll}\text { ITU } & \text { International Telecommunication Union } \\ \text { IETF } & \text { Internet Engineering Task Force } \\ \text { NTP } & \text { Network Time Protocol } \\ \text { PTP } & \text { Precision Time Protocol } \\ \text { MOS } & \text { Mean-Opinion-Score } \\ \text { DMOS } & \text { Degradation MOS } \\ \text { CMOS } & \text { Comparative MOS } \\ \text { ACR } & \text { Absolute Category Rating } \\ \text { CCR } & \text { Comparative Category Rating } \\ \text { DCR } & \text { Degradation Category Rating } \\ \text { PESQ } & \text { Perceptual Evaluation of Speech Quality } \\ \text { QoS } & \text { Quality of Service } \\ \text { QoE } & \text { Quality of Experience } \\ \text { CI } & \text { Confidence Interval } \\ \text { VOD } & \text { Video-On-Demand } \\ \text { CIF } & \text { Common Intermediate Format } \\ \text { QCIF } & \text { Quarter Common Intermediate Format } \\ \end{array}$




$\begin{array}{ll}\text { MNOG } & \text { Massively Multiplayer Online Game } \\ \text { CSCW } & \text { Computer Supported Cooperative Work } \\ \text { TCP } & \text { Transmission Control Protocol } \\ \text { UDP } & \text { User Datagram Protocol } \\ \text { IP } & \text { Internet Protocol } \\ \text { RTP } & \text { Real-time Transport Protocol } \\ \text { RTCP } & \text { Real-time Transport Control Protocol } \\ \text { IPTV } & \text { Internet Protocol based TV } \\ \text { VoIP } & \text { Voice over Inter Protocol } \\ \text { LAN } & \text { Local Area Network }\end{array}$

\section{List of Notations in Chapter 2:}

$t_{1} \quad$ Local time that a NTP packet leaves the NTP client

$t_{2} \quad$ Local time that a NTP packet arrives at the NTP server

$t_{3} \quad$ Local time that a NTP packet leaves the NTP server

$t_{4} \quad$ Local time that a NTP packet arrives at the NTP client

$\delta \quad$ Clock skew between NTP client and server

$k \quad$ Stratum layer index

$n \quad$ Number of user votes in subjective test

$X_{i} \quad$ Outcome of each user vote

$\bar{X} \quad$ Average of user votes

$\sigma \quad$ Sample variance

$n_{<0} \quad$ Number of votes that give a negative score

$n=0 \quad$ Number of votes that give a zero score

$n_{>0} \quad$ Number of votes that give a positive score

$n_{\leq 0} \quad$ Number of votes that give a non-positive score

$n_{\geq 0} \quad$ Number of votes that give a non-negative score

$p^{s} \quad$ Percentage of user votes for subjective test

$p \quad$ Probability value in hypothesis test

$\alpha \quad$ Significant level 


\section{CHAPTER 3}

\section{MODELING OF SYNCHRONIZATION SPECIFICATION}

The scalability of multimedia multi-device configurations in next-generation multi-site interactive systems requires a new model describing the emerging demands of multimedia synchronization specifications. In this chapter, we discuss the new multi-dimensional synchronization model, and investigate the time correlations for multimedia frames across the distributed media sensory devices.

\subsection{Mathematical Denotations}

We start with mathematical denotations. We are given a set of $N$ sites $\left(n^{1}, \ldots, n^{N}\right)$ in the distributed interactive multimedia systems, in which $N_{A P}$ sites are active participants in a media session, which send multimedia bundles to other sites. The media bundle produced by the sender site $n^{x}$ is denoted as $u^{x}$. A bundle $u^{x}$ is composed of $N M^{x}$ media modalities ${ }^{1}$, with each modality represented as $m_{i}^{x}\left(1 \leq i \leq N M^{x}\right)$, i.e., $u^{x}=\left\{m_{1}^{x}, \ldots, m_{N M^{x}}^{x}\right\}$. For example, we can use $i=1$ or $i=$ ' $\mathrm{V}$ ' to represent 3D multiview videos, $i=2$ or $i=$ 'A' for audios, $i=3$ or $i=$ 'B' for body sensors, $i=4$ or $i=$ 'H' for haptics, and etc. Each media modality $m_{i}^{x}$ consists of $N S_{i}^{x}$ streams with each stream indicated as $s_{i, j}^{x}\left(1 \leq j \leq N S_{i}^{x}\right)$, i.e., $m_{i}^{x}=\left\{s_{i, 1}^{x}, \ldots, s_{i, N S_{i}^{x}}^{x}\right\}$. A stream includes a set of media frames with the $k$-th frame indicated as $f_{i, j}^{x}(k)$ (i.e., $\left.s_{i, j}^{x}=\left\{f_{i, j}^{x}(1), \ldots, f_{i, j}^{x}(k), \ldots\right\}\right)$, and the average inter-frame period as $\Delta T_{i}$. Here, we assume all sensory streams $\left\{s_{i, j}^{x}\right\}$ belonging to the same media modality $m_{i}^{x}$ are shared with the same periodicity setting $\Delta T_{i}$.

Note that in the teleimmersion, a receiver site $n^{y}$ may only request a subset of multimedia streams within the bundle $u^{x}$ produced from the sender $n^{x}$. We denote this subset as the request bundle $r u^{x \rightarrow y}$, satisfying $r u^{x \rightarrow y} \in u^{x}$. There is a total of $N_{A P} \times N$ request bundles within a teleimmersive session.

\footnotetext{
${ }^{1}$ Throughout the dissertation, the superscript $x$ can be neglected when no specific sender site is indicated.
} 


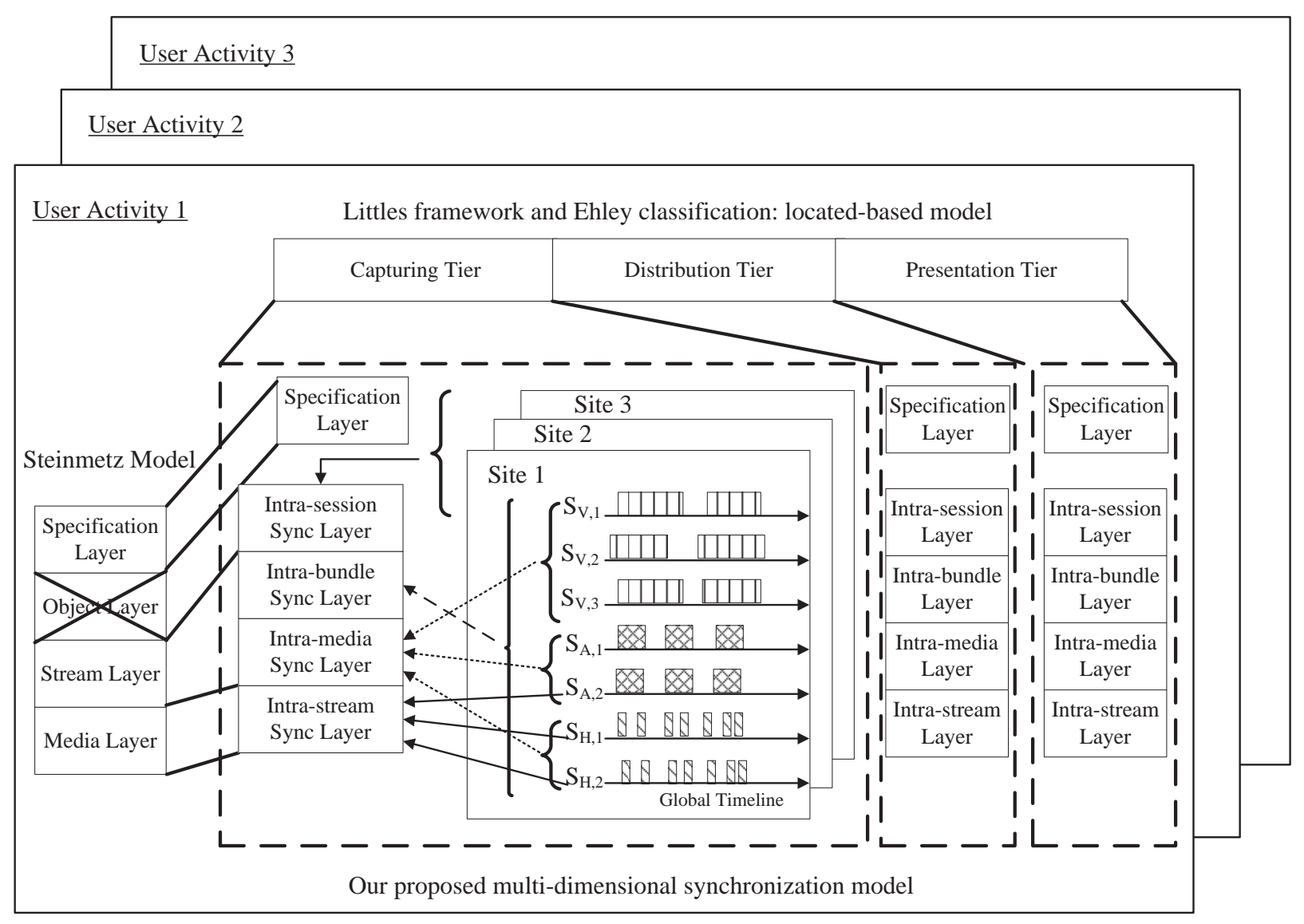

Figure 3.1: Our proposed multi-dimensional synchronization model.

\subsection{A Multi-dimensional Synchronization Model}

The multi-layer synchronization demands, the multi-location pipeline structure, and the activity-dependent synchronization reference hierarchy of multimedia systems drive the deciding dimensions of the overall synchronization quality. Fig. 3.1 shows the new multi-dimensional model we propose for the next-generation distributed interactive multimedia systems.

\subsubsection{Dimension of Synchronization Demands}

The first dimension describes the type of synchronization demands. The four synchronization layers that we have discussed in Section 1 (intra-stream, intra-media, intra-bundle and intra-session layers) are mainly derived and extended from previous Steinmetz's synchronization classification model [8,9] (Section 2). Specifically, the following factors need to be addressed.

- The media layer in Steinmetz's model is the same as the intra-stream layer in our study to preserve 
the time dependencies of media frames within each sensory stream.

- The inter-stream synchronization in their stream layer must now be divided into intra-media and intra-bundle layers separately to preserve media temporal-and-spatial correlations. The reason is that the new multimedia systems like teleimmersion can be equipped with camera arrays, microphone arrays and large number of body sensors, that output temporally-and-spatially correlated sensory streams for each continuous media modality. This correlation creates a new and strict intra-media synchronization requirement which was never investigated in previous studies. For example, if we need to synchronize two video streams from a camera array and one audio stream, which is the synchronization reference, Steinmetz's model can only specify in the stream layer that the two video streams must synchronize separately to the audio. But the skews between the two video streams can be unbounded which is unacceptable in multi-camera systems. That is why we must propose to add the intra-media layer and synchronize streams of the same modality with respect to their reference stream (selection algorithm will be discussed later). This has been neglected even in the work finished within the past 5-6 years [19], when camera/microphone arrays were being deployed, mainly because of the community's stereotyped view of synchronizing a single video and a single audio stream in the most common on-demand or conferencing multimedia systems.

- The (receiver) group synchronization in Steinmetz's stream layer must also be extended to cover both inter-sender and inter-receiver synchronization (i.e., the intra-session layer in our study), due to the fact that there are emerging demands for multiple sender sites to perform collaborative activities in multimedia systems like teleimmersion. Realizing the inter-sender synchronization can guarantee the interactivity of the users.

- Their object layer is removed from our study, mainly because we only focus on the continuous multimedia streams, assuming the synchronization between continuous and discrete media objects have been solved (Section 2).

- Steinmetz's specification layer is important, and that is the purpose of this chapter. But this layer does not belong to the dimension of the synchronization demands in our model.

\subsubsection{Dimension of Synchronization Locations}

The performance of the four-layer synchronization demands can change throughout media propagation in multiple locations in a multimedia system. Synchronization performance in previous locations can have a di- 
rect impact on future locations. Hence, an orthogonal location-based dimension is added to our synchronization model in order to describe the whole multimedia session. A good synchronization adaptation algorithm cannot be achieved without addressing the interaction between the synchronization demands and locations. This interaction is the direct extension to previous location-based Ehley's classification model [24], where only the inter-stream synchronization is addressed and evaluated in different synchronization locations.

\subsubsection{Dimension of Synchronization Reference Hierarchy}

The dimension of synchronization reference hierarchy is added to our model to describe the impact of the activity and application heterogeneity of multimedia systems on the human perception. As we have studied in our survey (Section 2), existing studies prescribe the selection of the synchronization reference stream/site without taking into account the activities and media application functionality, because they confine themselves to Skype-like conferencing applications with limited continuous media modalities and sensory streams (usually one audio and one video stream) from each site. Due to the multi-layer synchronization demands in next-generation multimedia systems like teleimmersion, it is not possible to use a single synchronization reference stream/site to represent a whole layered hierarchy. Each synchronization demand layer must identify its own reference, based upon the functionality of performed activities and end user interests.

\section{- L1: Intra-stream Layer}

The reference frame or the intra-stream synchronization reference is usually selected as the first media frame within a sensory stream at each system control update. We denote the reference frame of a sensory stream $s_{i, j}^{x}$ as $f_{i, j}^{x}(*)$.

\section{- L2: Intra-media Layer}

The intra-media synchronization reference is selected as the reference stream which has the largest contribution to end user interests within a media modality. The media contribution can vary depending on the characteristics of each modality. Here, we discuss four commonly deployed media modalities which we have used.

Multiview videos. Multiview video streams capture the same physical object at the same time, but from different viewpoints. The importance of each video stream is decided by their contributions of 3D image pixels to the end user viewpoint [12], which can be computed using the orientation difference between 
the sender camera and the receiver view. Given the sender $n^{x}$ 's camera orientation of a video stream $s_{V, j}^{x}$ (denoted as $\vec{O}\left(s_{V, j}^{x}\right)$ ), and the desired receiver $n^{y}$ 's view orientation from $n^{x}$ 's videos (denoted as $\vec{O}^{x, y}$ ), the visual contribution or the contribution factor $(\mathrm{CF})$ of $s_{V, j}^{x}$ to the receiver $n^{y}$ is [159]:

$$
\mathrm{CF}\left(s_{V, j}^{x}, n^{y}\right)=\vec{O}\left(s_{V, j}^{x}\right) \cdot \vec{O}^{x, y}
$$

Hence, the video reference stream $s_{V, *}^{x}$ is elected as the one with the largest CF in $m_{V}^{x}$ for each receiver.

Spatial audios. Multiple omni-directional microphones concurrently record the same physical ambient environment. The contribution of each audio stream is decided by its signal-to-noise ratio (SNR), a metric indicating the intelligibility of the speaker's utterances. SNR can be computed online by estimating the noises from the silence periods. We prescribe that the audio reference $s_{A, *}^{x}$ is the one with the largest SNR in $m_{A}^{x}$.

Haptics or Body sensory streams. Multiple haptic or body sensory streams may record different parts of a physical object. In our study, we decide the reference stream of each media modality $s_{B, *}^{x}$ or $s_{H, *}^{x}$ as the one with the largest data rate within $m_{B}^{x}$ or $m_{H}^{x}$, because a larger data rate for these sensory streams usually means higher-precision information.

\section{- L3: Intra-bundle Layer}

The importance of media modalities can vary at different activities, and the intra-bundle synchronization reference is defined as the most important reference modality. Empirically for teleimmersive applications, we can classify different activities based on real user perceptual feedback. (1) Users attach more importance to the intelligibility of audio signals in a conversation-oriented activity (e.g., conferencing or remote education), so the reference modality is the audio. (2) The clarity of video signals is of the greatest significance in a collaborative task with fine motor skills (e.g., rock-paper-scissor gaming or cyber-archeology), so the video is elected as the reference modality. (3) The body sensory streams can have the largest contribution in the telehealth or the remote rehabilitation activity, because the doctors need to evaluate the patient's health status by consistent body sensory feedback. Thus, we choose the body sensory modality as the reference. In our study, we denote the reference modality as $m_{*}^{x}$ for each bundle $u^{x}$.

\section{- L4: Intra-session Layer}

In multi-site interactive multimedia systems, the most active site usually demands higher-quality streaming bundles in order to guarantee uninterrupted collaborations in a session. The intra-session synchronization reference of inter-sender or inter-receiver synchronization is, thus, selected as the media bundle corre- 
sponding to the most active user within the sender/receiver group. In the teleimmersive system, for example, this user can usually take the lead in the activity (e.g., a trainer in the remote education, a director in the conferencing, or a doctor in the telehealth). The selection of the lead person is context-dependent, so it must be specified explicitly by the media applications. Here, we denote the reference site, or the intra-session reference as $n^{*}$.

\subsubsection{Definition of Synchronization Skews}

By consulting the above multi-dimensional synchronization model, we formulate the specifications in representing the multi-layer synchronization skews. We assume that the media data are sent to the receiver site $n^{y}$.

Intra-stream synchronization skew. The skew within a sensory stream $s_{i, j}^{x}$ is evaluated by computing the delay difference of a media frame $f_{i, j}^{x}(k)$ w.r.t. the reference frame $f_{i, j}^{x}(*)$. We denote $D\left(f_{i, j}^{x}(k), n^{y}\right)$ as the experienced latency of $f_{i, j}^{x}(k)$ from its captured time. Thus, the skew is defined as:

$$
\forall x, y, i, j: \Delta D\left(f_{i, j}^{x}(k), n^{y}\right)=D\left(f_{i, j}^{x}(k), n^{y}\right)-D\left(f_{i, j}^{x}(*), n^{y}\right)
$$

Intra-media synchronization skew. We denote $D\left(s_{i, j}^{x}, n^{y}\right)$ as the experienced latency of $s_{i, j}^{x}$. The intra-media synchronization skew $\Delta D\left(s_{i, j}^{x}, n^{y}\right)$ is defined as:

$$
\forall x, y, i, j: \Delta D\left(s_{i, j}^{x}, n^{y}\right)=D\left(s_{i, j}^{x}, n^{y}\right)-D\left(s_{i, *}^{x}, n^{y}\right)
$$

Intra-bundle synchronization skew Because sensory streams within a media modality can experience heterogeneous latencies, we prescribe that the latency of a media modality is equivalent to that of the intramedia synchronization reference within this modality, in order to best match human perceptual interests, i.e., $D\left(m_{i}^{x}, n^{y}\right)=D\left(s_{i, *}^{x}, n^{y}\right)$. Hence the intra-bundle synchronization skew of $m_{i}^{x}$ is defined as:

$$
\forall x, y, i: \quad \Delta D\left(m_{i}^{x}, n^{y}\right)=D\left(m_{i}^{x}, n^{y}\right)-D\left(m_{*}^{x}, n^{y}\right)
$$

Intra-session synchronization skew. Similar to intra-bundle layer, we prescribe that the latency of a bundle is equivalent to that of the intra-bundle synchronization reference within the bundle, i.e., $D\left(\mathscr{t}, n^{y}\right)=$ $D\left(m_{*}^{x}, n^{y}\right)$. Given the intra-session reference $n^{*}$, the sender group synchronization skew as to a receiver site $n^{y_{0}}$ is:

$$
\forall x: \Delta D\left(u^{x}, n^{y_{0}}\right)=D\left(u^{x}, n^{y_{0}}\right)-D\left(u^{n^{*}}, n^{y_{0}}\right)
$$

Accordingly, the receiver group synchronization skew as to a sender site $n^{x_{0}}$ is:

$$
\forall y: \Delta D\left(u^{x_{0}}, n^{y}\right)=D\left(u^{x_{0}}, n^{y}\right)-D\left(u^{x_{0}}, n^{*}\right)
$$




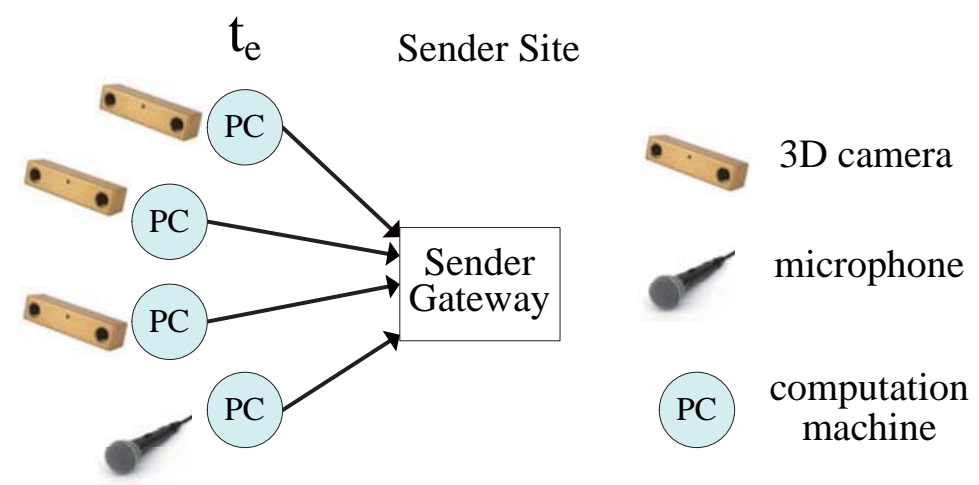

Figure 3.2: Illustration of media sensory devices and computation machines at the sender sites.

Remarks. The correlations of defining the hierarchical synchronization skews have two implications. First, at each location, the adaptive synchronization schemes in controlling the skews in one layer can directly impact those in other layers. Hence, previous single-reference synchronization control algorithms cannot work well in the activity- and application- specific multimedia systems without a knowledge of multi-layer synchronization dependencies and real human interests. Second, the synchronization skews of one layer in one location can also affect those of other layers in different locations. Hence, a new multilocation synchronization adaptation scheme is a must to address the interaction between the synchronization demands and locations, based upon activity-dependent synchronization references.

\subsection{Time Correlations of Multi-modal Media Frames}

Multi-modal media frames belonging to the same sensory stream may experience heterogeneous latencies from their captured time. In order to evaluate the multi-layer synchronization skews, we should explicitly prescribe specific media frames for each stream, such that their latencies can be computed for synchronization evaluations. The set of multi-modal media frames from multiple streams is usually time-correlated, and is often called a synchronization point (SP), whose concept was borrowed from Steinmetz's specification model in 1990 [58] (Section 2). Media frames belonging to each SP must be played at multimedia output devices in order to preserve their temporo-spatial correlations. However, the multimedia system and computation constraints can pose great challenges in locating the time-correlated media frames. In this section, we investigate the decision rules for the sequence of SP at each sender site, and propose metrics to evaluate their synchronization quality. 

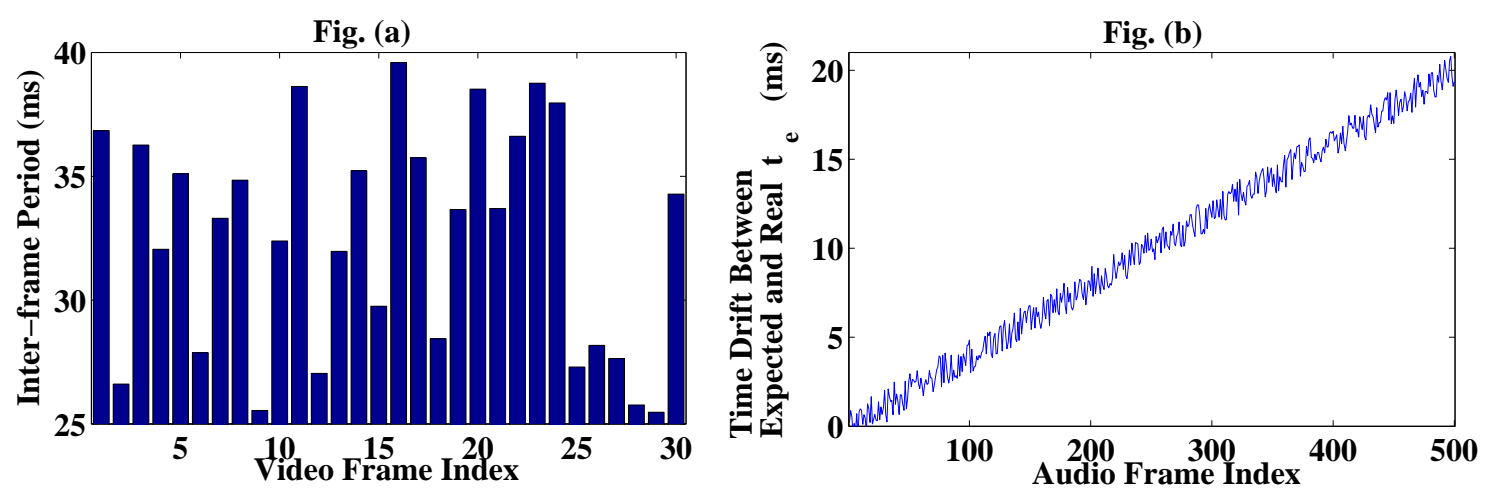

Figure 3.3: (a) Video inter-frame period; (b) Difference of expected and actual captured timestamp for a USB microphone with clock drift, measured at the USB sound card.

\subsubsection{System Background}

We start with a brief discussion of the multimedia system configuration and the impact on identifying the time correlations of media frames at the sender sites. Fig. 3.2 shows an illustration of a typical sender configuration for next-generation distributed interactive multimedia systems like teleimmersion. Usually, the computation machines attached to each multimedia sensory input device can be assumed clock synchronized by either the Network Time Protocol (NTP) [160] or the Precision Time Protocol (PTP) [117]. In other words, we assume that a global timeline can be accessed. The (global) time that a media frame is released by the sensory interface and it arrives at its specialized computation machine is called the actual captured timestamp, and is denoted as $t_{e}$ in our study.

We will use $t_{e}$ information to identify the time correlations of multi-modal media frames, but its characteristics in the real multimedia systems can complicate the problem.

1. Due to the hardware constraints, many media sensors can only capture media frames with weak periodicity. For example, a 30-Hz stereoscopic camera outputs multiview video streams with interframe period ranging from 25-40 ms (Fig. 3.3(a)).

2. A physical sampling drift can also appear in the media sensors. For example, a USB microphone with a $50-\mathrm{Hz}$ periodicity setting may only output 499 audio frames over a 10 -sec time span in reality. Fig. 3.3(b) shows the resulting time drift (i.e. the difference between expected $t_{e}$ and real $t_{e}$ ) (measured directly at the USB sound card) for each audio frame.

In this study, we will address these real system issues in deciding the SP at the sender sites. Previous studies $[161,162]$ located the time correlations of single-modal media frames by optimistically assuming their strong periodicity at the media source, and they did not manage to find the best timestamp describing 
the group of identified time-correlated media frames. [163] time tagged the image sequence with interframe period variations, but this study was only for a single camera. Hence, these works cannot be directly extended to the real multimedia configurations.

\subsubsection{Problem Formulation}

Our goal is to find the time dependencies across multi-modal media frames from distributed sensory devices at each sender site, group the time-correlated media frames into a SP, and assign these frames with the same captured timestamp. We denote the assigned captured timestamp as $t_{c}$. In other words, we aim to find the mapping $t_{e} \mapsto t_{c}$ for each media frame.

We prescribe that a SP sequence must be strongly periodic. The reasons are two fold. First, each sensory stream itself exhibits weakly periodicity. Second, a strongly periodic SP sequence can allow more predictable and manageable media traffic, and guarantee multimedia synchronization locations, because the synchronization statuses can be evaluated at regular intervals. Note that for the rest of this section, the term "periodic" always means "strongly periodic". We will explicitly mention "weakly periodic" when needed.

Because multiple media modalities can be characterized by different inter-frame periods, it is not possible to define a single periodic SP sequence to capture all media modalities with different periodic intervals. We address the issue by designing the SP in a hierarchical fashion. At each sender site, we first investigate the problem of intra-media synchronization point (SP), i.e., the set of time-correlated media frames belonging to the same media modality (Fig. 3.4), and one media frame from each same-modal sensory stream. Based on the identified sequences of intra-media SP for each media modality, we then locate the set of intra-bundle synchronization point (SP), i.e., the set of time-correlated multi-modal intra-media SP sourced at the same site (Fig. 3.5), and each intra-media SP from different media modalities.

\section{- Intra-media Synchronization Point}

W.L.O.G, we study a specific sender site $n^{x_{0}}$ and one of its media modality $i_{0}$. The set of sensory

streams $\left\{s_{i_{0}, j}^{x_{0}}\right\}$ is configured with a weak periodicity setting with an average frame period $\Delta T_{i_{0}}$. We have the following two targets:

1. Find the periodic intra-media SP sequence $\left\{s p_{i_{0}}^{x_{0}}(\hat{k})\right\}$ with the same period $\Delta T_{i_{0}}$, where each intramedia SP contains one and only one media frame from each sensory stream $s_{i_{0}, j}^{x_{0}}(\forall j)$. To facilitate the illustrations and minimize the confusions, we use $\hat{k}$ to indicate the index of intra-media SP and 


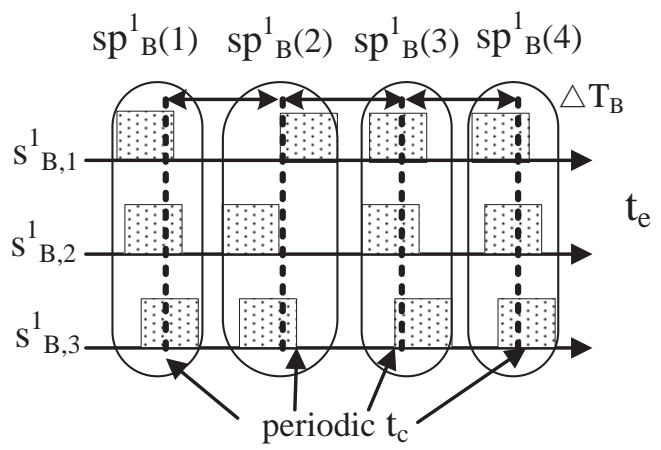

Figure 3.4: An example of intra-media SP sequence for three body sensory streams. Each solid block indicates a media frame. The solid circular rectangles represent the intra-media SP sequence, whose assigned captured timestamp $t_{c}$ has a periodicity of $\Delta T_{B}$.

$k$ for the index of media frames. Fig. 3.4 shows several intra-media SP represented in solid circular rectangles.

2. Decide the best assigned captured timestamp for each intra-media SP: $t_{c}\left(s p_{i_{0}}^{x_{0}}(\hat{k})\right)$, in order to minimize the time relabeling error of multi-modal media frames. The dotted lines in Fig. 3.4 depict the resulting periodic $t_{c}$.

Assuming $\left.k\right|_{j}$ is the frame index for stream $j$, the above two targets can be described as follows.

$$
\begin{array}{cl}
\text { Inputs : } & x_{0}, i_{0} \\
& t_{e}\left(f_{i_{0}, j}^{x_{0}}\left(\left.k\right|_{j}\right)\right) \\
\text { Outputs : } \quad & s p_{i_{0}}^{x_{0}}(\hat{k}), t_{c}\left(s p_{i_{0}}^{x_{0}}(\hat{k})\right) \\
& \hat{k} \mapsto\left\{\left.k\right|_{1},\left.k\right|_{2}, \ldots\right\} \\
\text { Optimization : } \quad & \min \sum_{c} \sum_{j}\left|t_{c}\left(s p_{i_{0}}^{x_{0}}(\hat{k}+c)\right)-t_{e}\left(f_{i_{0}, j}^{x_{0}}\left(\left.k\right|_{j}+c\right)\right)\right| \\
\text { Constraints : } \quad \forall \text { constant integer } c: & s p_{i_{0}}^{x_{0}}(\hat{k}+c)=\left\{f_{i_{0}, 1}^{x_{0}}\left(\left.k\right|_{1}+c\right), f_{i_{0}, 2}^{x_{0}}\left(\left.k\right|_{2}+c\right), \ldots\right\} \\
& t_{c}\left(s p_{i_{0}}^{x_{0}}(\hat{k}+c)\right)=t_{c}\left(s p_{i_{0}}^{x_{0}}(\hat{k})\right)+(c-1) \times \Delta T_{i_{0}}
\end{array}
$$

\section{- Intra-bundle Synchronization Point}

Given the set of periodic intra-media SP sequences $\left\{s p_{i}^{x_{0}}(\hat{k})\right\}$ for each media modality $i$, we decide the resulting set of intra-bundle SP at each site. Similar to the intra-media SP problem, we also have two goals in identifying the intra-bundle SP sequence. 


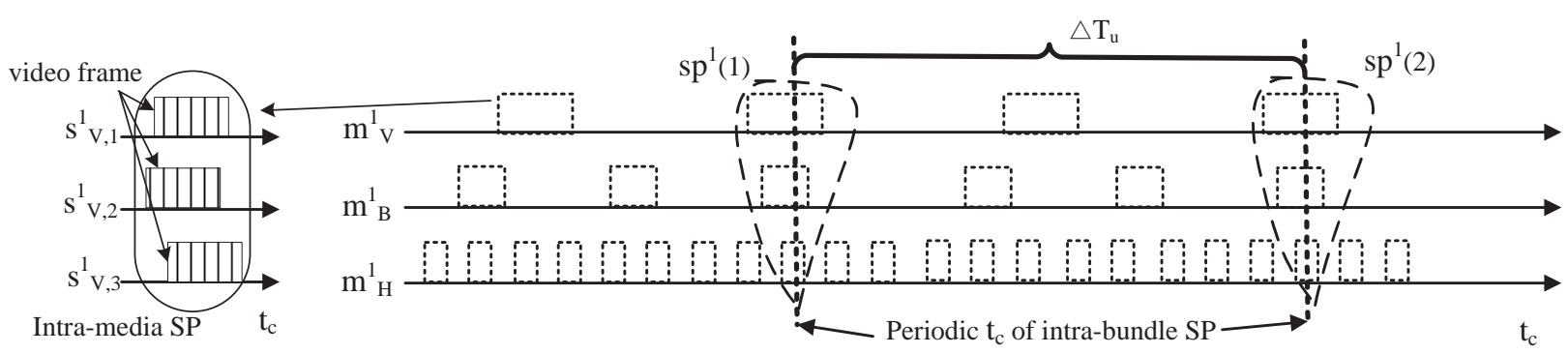

Figure 3.5: An example of intra-bundle SP sequence. Each dotted block indicates an intra-media SP. The dotted surrounding curves represent the intra-bundle SP sequence, whose assigned captured timestamp $t$ has a periodicity of $\Delta T_{u}$.

1. Find the periodic intra-bundle SP sequence $\left\{s p^{x_{0}}(\hat{l})\right\}$ with the period $\Delta T_{u}$, where each intra-bundle SP contains one and only one intra-media SP from each media modality $i$. Here, we use $\hat{l}$ to indicate the index of the intra-bundle SP. Due to different periodicity of intra-media SP sequences, $\Delta T_{u}$ must be equivalent to the least common multiplier of all possible intra-media periods, i.e., $\Delta T_{u}=\mathrm{LCM}\left(\Delta T_{1}, \Delta T_{2}, \ldots\right)$. As an example, Fig. 3.5 shows the intra-bundle SP sequence represented by the dotted surrounding curves.

2. Decide the best assigned captured timestamp for each intra-bundle SP: $t_{c}\left(s p^{x_{0}}(\hat{l})\right)$, in order to minimize the time relabeling error of multi-modal intra-media SP. The dotted lines in Fig. 3.5 depict the resulting periodic $t_{c}$ of the intra-bundle SP sequence.

Assuming $\left.\hat{k}\right|_{i}$ is the intra-media SP index for media modality $i$, the above two goals can be represented as:

$$
\begin{array}{cl}
\text { Inputs : } & x_{0} \\
& t_{c}\left(s p_{i}^{x_{0}}\left(\left.\hat{k}\right|_{i}\right)\right) \\
& s p^{x_{0}}(\hat{l}), t_{c}\left(s p^{x_{0}}(\hat{l})\right) \\
& \hat{l} \mapsto\left\{\left.\hat{k}\right|_{1},\left.\hat{k}\right|_{2}, \ldots\right\} \\
\text { Outputs : } & \min \sum_{c} \sum_{i}\left|t_{c}\left(s p^{x_{0}}(\hat{l}+c)\right)-t_{c}\left(s p_{i}^{x_{0}}\left(\left.\hat{k}\right|_{i}+\frac{\Delta T_{u} \times c}{\Delta T_{i}}\right)\right)\right| \\
\text { Constraints : } \quad \forall \operatorname{constant} \text { integer } c: & s p^{x_{0}}(\hat{l}+c)=\left\{s p_{1}^{x_{0}}\left(\left.\hat{k}\right|_{1}+\frac{\Delta T_{u} \times c}{\Delta T_{1}}\right), s p_{2}^{x_{0}}\left(\left.\hat{k}\right|_{2}+\frac{\Delta T_{u} \times c}{\Delta T_{2}}\right), \ldots\right\} \\
& t_{c}\left(s p^{x_{0}}(\hat{l}+c)\right)=t_{c}\left(s p^{x_{0}}(\hat{l})\right)+(c-1) \times \Delta T_{u}
\end{array}
$$




\subsubsection{Design Rationales}

\section{- Intra-media Synchronization Point (SP)}

First, the irregularity of inter-frame intervals (Fig. 3.6(a)) in the real multimedia systems can impede us to efficiently locate the intra-media SP sequence and find the best assigned captured timestamp correspondingly. Hence, the first step is usually to realign the original media frames of each sensory stream into a periodic sequence, as described in Fig. 3.6(b).

Second, the time-realigned periodic multi-stream data may still introduce a number of intra-media SP decision options which lead to different lengths of intra-media synchronization regions (SR) (i.e., the duration between the earliest and latest realigned media frames within each intra-media SP). For example, as Fig. 3.6(b) and (c) suggest, Option 2 decides an intra-media SP (covering media frames $k_{1}, k_{2}$ and $k_{3}$ ) which leads to a shorter SR compared Option 1 (covering media frames $k_{1}, k_{2}^{\prime}=k_{2}+1$ and $k_{3}$ ). It can easily be concluded without proof that a larger SR can cause a greater time relabeling error. Thus, a good algorithm should find the option which can minimize the corresponding SR length.

\section{- Intra-bundle Synchronization Point (SP)}

The scalability of media modalities can also offer numerous intra-bundle SP decision options with different lengths of intra-bundle synchronization regions (SR) (i.e., the duration between the earliest and latest intra-media SP that belong to the same intra-bundle SP). Fig. 3.7 describes two intra-bundle SP options, where Option 2 leads to a smaller SR. To find the best assigned captured timestamp with a minimal time relabeling error, we should choose the intra-bundle SP option with a minimal SR duration.

\subsubsection{Identification Algorithm for Intra-media SP}

\section{- Identification Algorithm}

Step 1. For each sensory stream, we perform time realignment of media frames and estimate their periodic expected captured time $t_{e}^{\prime}$ in order to smooth the irregularity of inter-frame intervals. $t_{e}$ can be computed based on $\Delta T_{i_{0}}$ and $t_{e}$. Specifically, for the sender site $n^{x_{0}}$ and its media modality $i_{0}$, the $\left.k\right|_{j}$-th media frame of the $j$-th sensory stream $f_{i_{0}, j}^{x_{0}}\left(\left.k\right|_{j}\right)$ is with $t_{e}^{\prime}$ :

$$
t_{e}^{\prime}\left(f_{i_{0}, j}^{x_{0}}\left(\left.k\right|_{j}\right)\right)=\left(\left.k\right|_{j}-1\right) \times \Delta T_{i_{0}}+\text { offset }_{i_{0}, j}^{x_{0}}
$$

where offset $x_{i_{0}, j}^{x_{0}}$ is the time offset of the first media frame $f_{i_{0}, j}^{x_{0}}(1)$, and can be computed at each system 

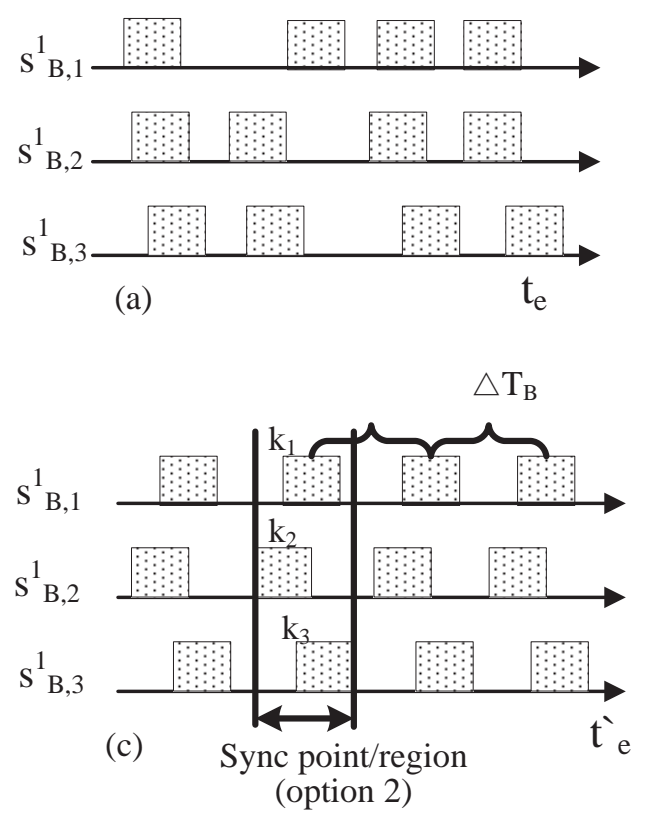

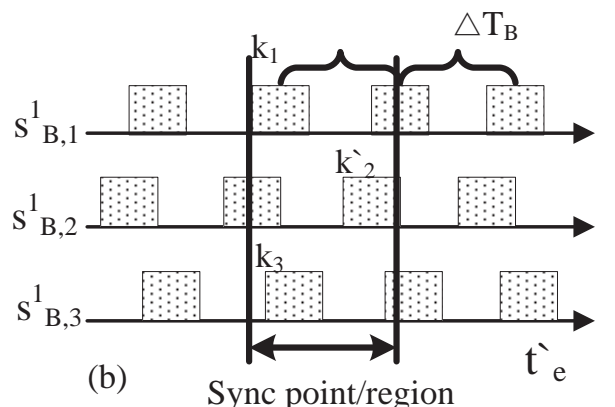

(option 1)

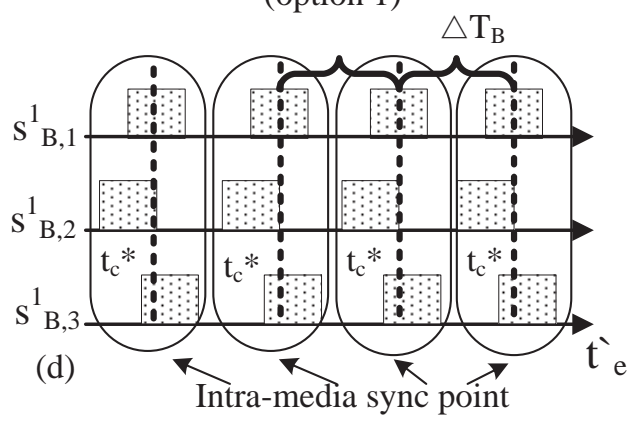

Figure 3.6: Two options of intra-media SP/SR (between solid lines) and resulting best assigned captured timestamp $t_{c}^{*}$ (dotted lines) for body sensory streams. Each block indicates a media frame.

update by linear regression based on the actual captured time $t_{e}$ of all media frames within the past timing window, i.e.,

$$
\text { offset }_{i_{0}, j}^{x_{0}}=\arg \min _{q} \sum_{\left.k\right|_{j}}\left[t_{e}\left(f_{i_{0}, j}^{x_{0}}\left(\left.k\right|_{j}\right)\right)-\left(\left.k\right|_{j}-1\right) \times \Delta T_{i_{0}}-q\right]^{2}
$$

In the above equation, $q$ is the variable representing different possible values of offse $\tau_{0}^{x_{0}}{ }^{2}$.

W.L.O.G, we assume the following condition is satisfied in our analytical model:

$$
\begin{gathered}
\max _{j}\left\{\operatorname{offset}_{i_{0}, j}^{x_{0}}\right\}-\min _{j}\left\{\operatorname{offset}_{i_{0}, j}^{x_{0}}\right\}<\Delta T_{i_{0}} \\
\forall j<j^{\prime} \quad \operatorname{offset}_{i_{0}, j}^{x_{0}}<\text { offset }_{i_{0}, j^{\prime}}^{x_{0}}
\end{gathered}
$$

Step 2. Given the time realigned media sequence, for each sensory stream $j$, and its media frame $f_{i_{0}, j}^{c_{0}}(1)$ (i.e., the first media frame of the $j$-th stream), we can identify an intra-media SP whose left bound (earliest time $)$ is $\left.t^{\text {left }}\right|_{j}=t_{e}^{\prime}\left(f_{i_{0}, j}^{x_{0}}(1)\right)$, and right bound $\left.t^{\text {right }}\right|_{j}$ is:

$$
\left.t^{\text {right }}\right|_{j}=\left\{\begin{array}{cl}
t_{e}^{\prime}\left(f_{i_{0}, N S_{i_{0}}^{x_{0}}}^{x_{0}}(1)\right) & \text { for } \quad j=1 \\
t_{e}^{\prime}\left(f_{i_{0}, j-1}^{x_{0}}(2)\right) & \text { for } \quad j=2, \ldots, N S_{i_{0}}^{x_{0}}
\end{array}\right.
$$

Hence, the corresponding SR length is equal to $\left.t^{\text {right }}\right|_{j}-\left.t^{\text {left }}\right|_{j}$. Due to the periodicity of realigned media frames, such an intra-media SP can actually decide one option of intra-stream SP sequence with the same 
SR length. It is not difficult to prove that the number of intra-media SP options is equivalent to the number of sensory streams belonging to the media modality $i_{0}$. We then pick the option with the minimal SR length, such that the time relabeling error can be minimized:

$$
\left.\min _{j} t^{\text {right }}\right|_{j}-\left.t^{\text {left }}\right|_{j}
$$

Step 3. Based on the identified intra-media SP sequence, we then decide the best assigned captured timestamp for each intra-media SP. We suppose a SP $s p_{i_{0}}^{x_{0}}(\hat{k})$ constitutes the set of media frames $\left\{f_{i_{0}, j}^{x_{0}}\left(\left.k\right|_{j}\right)\right\}$ (i.e., the $\left.k\right|_{j}$-the media frame of the $j$-th sensory stream). The best assigned captured timestamp $t_{c}^{*}\left(s p_{i_{0}}^{x_{0}}(\hat{k})\right)$ can be computed as:

$$
t_{c}^{*}\left(s p_{i_{0}}^{x_{0}}(\hat{k})\right)=\arg \min _{t} \sum_{j}\left|t-t_{e}^{\prime}\left(f_{i_{0}, j}^{x_{0}}\left(\left.k\right|_{j}\right)\right)\right|
$$

Here, we conclude the following two propositions.

Proposition 3.3.1 Suppose there is a total of $N S=N S_{i_{0}}^{x_{0}}$ media frames within each intra-media SP. If $N S$ is odd, $t_{c}^{*}$ is equivalent to the median value of $\left\{t_{e}^{\prime}\left(f_{i_{0}, 1}^{x_{0}}\left(\left.k\right|_{1}\right)\right), \ldots, t_{e}^{\prime}\left(f_{i_{0}, N S}^{x_{0}}\left(\left.k\right|_{N S}\right)\right)\right\}$. If $N S_{i_{0}}^{x_{0}}$ is even, $t_{c}^{*}$ is the average of the two median numbers.

Proof. W.L.O.G, we assume $\left\{t_{e}^{\prime}\left(f_{i_{0}, 1}^{x_{0}}\left(\left.k\right|_{1}\right)\right), \ldots, t_{e}^{\prime}\left(f_{i_{0}, N S}^{x_{0}}\left(\left.k\right|_{N S}\right)\right)\right\}$ is an incremental ordered list. We first prove the case of an odd $N S$. The median value is, thus, $t_{e}^{\prime}\left(f_{i_{0}, \frac{N S+1}{2}}^{x_{0}}\left(\left.k\right|_{\frac{N S+1}{2}}\right)\right)$. We let $t_{c}^{*}=$ $t_{e}^{\prime}\left(f_{i_{0}, \frac{N S+1}{2}}^{x_{0}}\left(\left.k\right|_{\frac{N S+1}{2}}\right)\right)$ and $t_{c}^{\prime}=t_{c}^{*}+\epsilon=t_{e}^{\prime}\left(f_{i_{0}, \frac{N S+1}{2}}^{x_{0}}\left(\left.k\right|_{\frac{N S+1}{2}}\right)\right)+\epsilon \quad(\epsilon \neq 0)$. Hence, the following inequations must satisfy:

$$
\begin{aligned}
\left|t_{e}^{\prime}\left(f_{i_{0}, 1}^{x_{0}}\left(\left.k\right|_{1}\right)\right)-t_{c}^{*}\right|+\left|t_{e}^{\prime}\left(f_{i_{0}, N S}^{x_{0}}\left(\left.k\right|_{N S}\right)\right)-t_{c}^{*}\right| & \leq\left|t_{e}^{\prime}\left(f_{i_{0}, 1}^{x_{0}}\left(\left.k\right|_{1}\right)\right)-t_{c}^{\prime}\right|+\left|t_{e}^{\prime}\left(f_{i_{0}, N S}^{x_{0}}\left(\left.k\right|_{N S}\right)\right)-t_{c}^{\prime}\right| \\
\left|t_{e}^{\prime}\left(f_{i_{0}, 1}^{x_{0}}\left(\left.k\right|_{2}\right)\right)-t_{c}^{*}\right|+\left|t_{e}^{\prime}\left(f_{i_{0}, N S-1}^{x_{0}}\left(\left.k\right|_{N S-1}\right)\right)-t_{c}^{*}\right| & \leq\left|t_{e}^{\prime}\left(f_{i_{0}, 2}^{x_{0}}\left(\left.k\right|_{2}\right)\right)-t_{c}^{\prime}\right|+\left|t_{e}^{\prime}\left(f_{i_{0}, N S-1}^{x_{0}}\left(\left.k\right|_{N S-1}\right)\right)-t_{c}^{\prime}\right|
\end{aligned}
$$

We can conclude that $t_{c}^{*}$, the median number, will lead to the minimal relabeling errors for all media frames within the identified intra-media SP, when $N S$ is odd. Similarly, in the case of an even $N S$, we can also prove that $t_{c}^{*}$ can be any value between the two median numbers: $t_{e}^{\prime}\left(f_{i_{0}, N S / 2}^{x_{0}}\left(\left.k\right|_{N S / 2}\right)\right)$ and $t_{e}^{\prime}\left(f_{i_{0}, N S / 2+1}^{x_{0}}\left(\left.k\right|_{N S / 2+1}\right)\right)$. Hence, we pick their average in our study.

Proposition 3.3.2 Within each identified intra-media SP (i.e., the SP with the minimal SR length), the time relabeling error of each media frame must be no greater than $\Delta T_{i_{0}} / 2$, i.e., $\left|t_{e}^{\prime}\left(f_{i_{0}, j}^{x_{0}}\left(\left.k\right|_{j}\right)\right)-t_{c}^{*}\left(s p_{i_{0}}^{x_{0}}(\hat{k})\right)\right| \leq$ $\Delta T_{i_{0}} / 2\left(\forall j: f_{i_{0}, j}^{x_{0}}\left(\left.k\right|_{j}\right) \in s p_{i_{0}}^{x_{0}}(\hat{k})\right)$. 


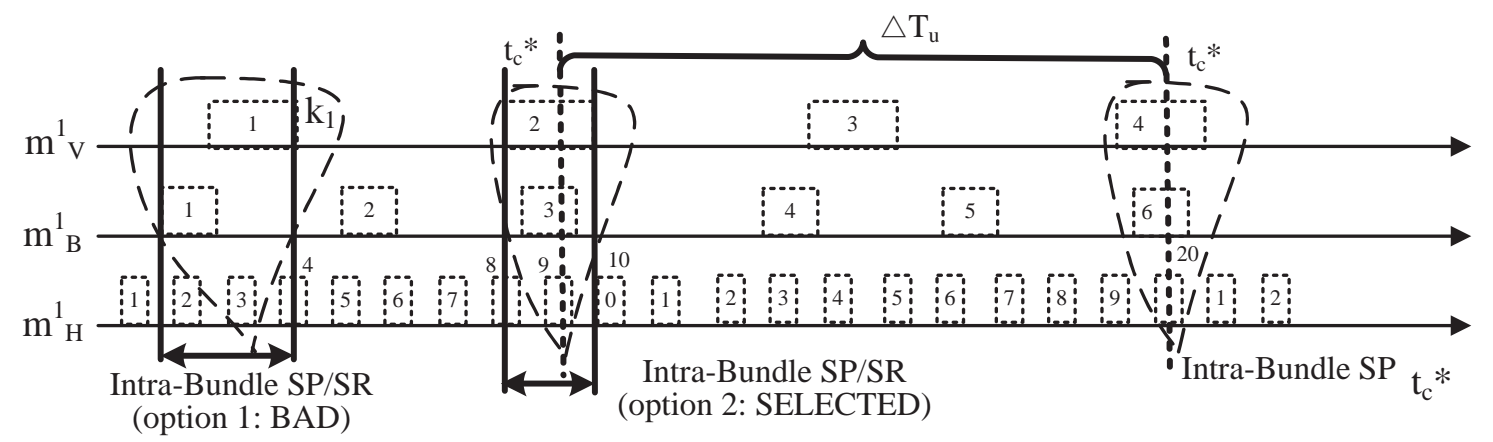

Figure 3.7: Two options of intra-bundle SP. Each dotted block indicates an intra-media SP.

Proof. This can be proved by contradiction. We assume there is a media frame $f_{i_{0}, j^{\prime}}^{x_{0}}\left(k^{\prime}\right)$ within the identified intra-media SP $s p_{i_{0}}^{x_{0}}\left(\hat{k}^{\prime}\right)$, such that the resulting time relabeling error is greater than $\Delta T_{i_{0}} / 2$, and is the largest error among all media frames within $s p_{i_{0}}^{x_{0}}\left(\hat{k}^{\prime}\right)$. Hence, there must be a neighbor media frame of the same sensory stream (i.e., either $f_{i_{0}, j^{\prime}}^{x_{0}}\left(k^{\prime}-1\right)$ or $\left.\left.f_{i_{0}, j^{\prime}}^{x_{0}}\left(k^{\prime}+1\right)\right)\right)$, such that the new set of media frames: $\left\{s p_{i_{0}}^{x_{0}}\left(\hat{k}^{\prime}\right)-f_{i_{0}, j^{\prime}}^{x_{0}}\left(k^{\prime}\right)+f_{i_{0}, j^{\prime}}^{x_{0}}\left(k^{\prime} \pm 1\right)\right\}$ (i.e., excluding the original media frame $f_{i_{0}, j^{\prime}}^{x_{0}}\left(k^{\prime}\right)$ but including one of its neighbor frames) will lead to an alternative intra-media SP option that has a smaller synchronization region than original identified intra-media SP. Hence, we reach a contradiction.

\section{- Decision Rule}

Based on the above discussions, we employ the following decision rule to identify the intra-media SP for real media data with irregular inter-frame intervals.

For any sender site $n^{x}$ and its media modality $i$, the $\left.k\right|_{j}$-th media frame $f_{i, j}^{x}\left(\left.k\right|_{j}\right)$ of the sensory stream $j$ belonging to an intra-media $\operatorname{SP} s p_{i}^{x}(\hat{k})$ must satisfy:

$$
t_{c}^{*}\left(s p_{i}^{x}(\hat{k})\right)-\Delta T_{i} / 2 \leq t_{e}^{\prime}\left(f_{i, j}^{x}\left(k_{j}\right)\right) \leq t_{c}^{*}\left(s p_{i}^{x}(\hat{k})\right)+\Delta T_{i} / 2
$$

We prescribe that $t_{c}\left(f_{i, j}^{x}\left(\left.k\right|_{j}\right)\right)=t_{c}^{*}\left(s p_{i}^{x}(\hat{k})\right)$ for all media frames belonging to the intra-media SP. In other words, if we suppose $s p_{i}^{x}(\hat{k})=\left\{f_{i, 1}^{x}\left(\left.k\right|_{1}\right), f_{i, 2}^{x}\left(\left.k\right|_{2}\right), \ldots\right\}$, we can obtain $t_{c}^{*}\left(s p_{i}^{x}(\hat{k})\right)=t_{c}\left(f_{i, 1}^{x}\left(\left.k\right|_{1}\right)\right)=$ $t_{c}\left(f_{i, 2}^{x}\left(\left.k\right|_{2}\right)\right)=\ldots$ Fig. $3.6(\mathrm{~d})$ shows 4 consecutive periodic intra-media SP with assigned captured time indicated in dotted lines.

\subsubsection{Identification Algorithm for Intra-bundle SP}

\section{- Identification Algorithm}

W.L.O.G, we assume that, at any site $n^{x_{0}},\left\{\Delta T_{1}, \Delta T_{2}, \ldots\right\}$ is an ordered list, i.e., $\Delta T_{1}>\Delta T_{2}>\cdots>$ 
$\Delta T_{N M^{x_{0}}}$. It is obvious that, within any arbitrary duration $t_{T}=\left(t, t+\Delta T_{u}\right)$, the number of intra-media SP for the media modality 1 with the largest period $\Delta T_{1}$ (i.e., $\left.t_{c}^{*}\left(s p_{1}^{x_{0}}\left(\left.\hat{k}\right|_{1}\right)\right) \in t_{T}\right)$ is equivalent to the number of the intra-bundle SP sequence options. We solve the problem using the following two steps.

Step 1. For each $s p_{1}^{x_{0}}\left(\left.\hat{k}\right|_{1}\right)$ within $t_{T}$, we enumerate, at each media modality (except $i=1$ ), the two intra-media SP whose $t_{c}^{*}$ that are immediately left and right to the time $t_{c}^{*}\left(s p_{1}^{x_{0}}\left(\left.\hat{k}\right|_{1}\right)\right)$. We then update the resulting intra-bundle SR length. For example, in Option 1 of Fig. 3.7, for the case of $s p_{1}^{x_{0}}(1)$, we need to enumerate over $s p_{2}^{x_{0}}(1)$ and $s p_{2}^{x_{0}}(2)$ for media modality $i=2$, and $s p_{3}^{x_{0}}(3)$ and $s p_{3}^{x_{0}}(4)$ for media modality $i=3$, in order to cover all possibilities completely. Hence, we need a total of 4 comparisons for 3 media modalities. We select the combination that leads to the minimal intra-bundle SR length as one candidate intra-bundle SP option, which, in Fig. 3.7, includes $\left\{s p_{1}^{x_{0}}(1), s p_{2}^{x_{0}}(1), s p_{3}^{x_{0}}(1)\right\}$ (Option 1).

We then iterate all $s p_{1}^{x_{0}}\left(\left.\hat{k}\right|_{1}\right)$ within $t_{T}$ to obtain all possible intra-bundle SP options. We select the one with the minimal SR length as the best intra-bundle SP. For example, we iterate between $s p_{1}^{x_{0}}(1)$ and $s p_{1}^{x_{0}}(2)$ in Fig. 3.7, and select the intra-bundle SP that contains $s p_{1}^{x_{0}}(2)$ (Option 2), and denote the selected intra-bundle SP as $s p^{x_{0}}(\hat{l})$.

Step 2. We follow Proposition 3.3.1 and 3.3.2, and prescribe that the best assigned captured timestamp $t_{c}^{*}\left(s p^{x_{0}}(\hat{l})\right)$ for the intra-bundle SP $s p^{x_{0}}(\hat{l})$ is the mean $t_{c}$ value of all -intra-media SP within $s p^{x_{0}}(\hat{l})$, in order to minimize the time relabeling error. For all intra-media SP belonging to $s p^{x_{0}}(\hat{l})$, we assign them with the same captured timestamp $t_{c}^{*}\left(s p^{x}(\hat{l})\right)$. For example, we suppose the first intra-bundle SP $s p^{x_{0}}(1)=$ $\left\{s p_{1}^{x_{0}}(2), s p_{2}^{x_{0}}(2), s p_{3}^{x_{0}}(9)\right\}$ in Fig. 3.7. Hence, $t_{c}^{*}\left(s p^{x_{0}}(1)\right)=t_{c}^{*}\left(s p_{1}^{x_{0}}(2)\right)=t_{c}^{*}\left(s p_{2}^{x_{0}}(2)\right)=t_{c}^{*}\left(s p_{3}^{x_{0}}(9)\right)$.

Note that we also need to adjust accordingly the captured timestamp of all other intra-media SP that does not belong to any intra-bundle SP. For example in Fig. 3.7, we need to relabel the time for both $s p_{1}^{x_{0}}(1)$ and $s p_{1}^{x_{0}}(3)$, such that $t_{c}^{*}\left(s p_{1}^{x_{0}}(1)\right)=t_{c}^{*}\left(s p_{1}^{x_{0}}(2)\right)-\Delta T_{1}$ and $t_{c}^{*}\left(s p_{1}^{x_{0}}(3)\right)=t_{c}^{*}\left(s p_{1}^{x_{0}}(1)\right)+\Delta T_{1}$.

Proposition 3.3.3 Within each identified intra-bundle SP (i.e., the SP with the minimal SR length), the maximum time relabeling error of each intra-media $S P$ must be no greater than $\Delta T_{1} / 2$, where $\Delta T_{1}$ is the largest inter-frame period.

Proof. This can be proved by contradiction. We assume there is an intra-media SP $s p_{i^{\prime}}^{x_{0}}\left(\hat{k}^{\prime}\right)$ within the identified intra-bundle SP $s p^{x_{0}}\left(\hat{l}^{\prime}\right)$, such that the resulting time relabeling error is greater than $\Delta T_{1} / 2$, and is the largest error among all intra-media SP within $s p^{x_{0}}\left(\hat{l}^{\prime}\right)$. Hence, there must be a neighbor intra-media SP of the same media modality (i.e., either $s p_{i^{\prime}}^{x_{0}}\left(\hat{k}^{\prime}-1\right)$ or $\left.s p_{i^{\prime}}^{x_{0}}\left(\hat{k}^{\prime}+1\right)\right)$, such that the new combinations of multi-modal intra-media SP: $\left\{s p^{x_{0}}\left(\hat{l}^{\prime}\right)-s p_{i^{\prime}}^{x_{0}}\left(\hat{k}^{\prime}\right)+s p_{i^{\prime}}^{x_{0}}\left(\hat{k}^{\prime} \pm 1\right)\right\}$ (i.e., excluding the original intra-media 
$\mathrm{SP} s p_{i^{\prime}}^{x_{0}}\left(\hat{k}^{\prime}\right)$ but including its neighbor intra-media SP) will lead to an alternative intra-bundle SP option that has a smaller SR length than original identified intra-bundle SP. Hence, we reach a contradiction.

\section{- Decision Rule}

Once an intra-bundle SP $s p^{x_{0}}(\hat{l})$ is decided, the periodic intra-bundle SP sequence can be decided by spacing $\Delta T_{u}$ apart between two consecutive intra-bundle SP. This can be achieved by counting the number of periodic intra-media SP within $\Delta T_{u}$ for each media modality. For example in Fig. 3.7, the second intrabundle SP is $s p^{x_{0}}(2)=\left\{s p_{1}^{x_{0}}(4), s p_{2}^{x_{0}}(6), s p_{3}^{x_{0}}(20)\right\}$. The assigned captured time $t_{c}^{*}\left(s p^{x_{0}}(2)\right)$ is equivalent to $t_{c}^{*}\left(s p^{x_{0}}(1)\right)+\Delta T_{u}$.

\section{- Computation Complexity}

As the number of media modalities $N M\left(N M=N M^{x_{0}}\right)$ scales at each sender, it is easy to show that the worst (largest) number of comparisons in Step 1 can be as much as $2^{N M-1}$ (an exponential complexity). Here, we investigate the best case: the minimal number of comparisons needed. The behind rationale is that the enumeration can stop at a media modality $i$ when its period $\Delta T_{i}$ is less than the intra-bundle SR length decided only by media modalities 1 to $i-1$, because the intra-bundle SR length can no longer be increased when new modalities are further added.

Theorem 3.3.4 The earliest time that the enumeration stops is at $i_{\min }$, where $i_{\min }=\arg \min _{i}\left\{\Delta T_{i}<\right.$ $\left.\left(\Delta T_{1}-\Delta T_{1} / 2^{i-2}\right)\right\}(i \geq 3)$, assuming $\left\{\Delta T_{i}\right\}$ is an ordered list.

Proof. Because $\Delta T_{i}$ is monotonically non-increasing as $i$ increases, after our algorithm enumerates and compares the intra-media SP at media modality up to $i-1$, the maximum-possible length of the minimal intra-bundle SR must be less than or equal to $\Delta T_{1}-\Delta T_{1} / 2^{i-2}$ [161]. The equivalence satisfies only when $\Delta T_{1}=\Delta T_{2}=\cdots=\Delta T_{i-1}$. Hence, when $\Delta T_{i}$ is less than the intra-bundle SR decided for media modalities up to $i-1$, there must be an intra-media SP within this region, and the enumeration stops.

\subsubsection{Impact of Real System Challenges}

We evaluate the quality of intra-bundle and intra-media SP sequence using the synchronization efficiency. It covers two aspects: (1) time efficiency, specifying the time that a resynchronization is needed; (2) accuracy efficiency, evaluating how well the media data are synchronized. The concept of the synchronization efficiency was originally designed for synchronizing concurrent processes in the operating system [164]. 
Here, we extend the concept for continuous media synchronization in the distributed interactive multimedia systems.

\section{- System Model}

We investigate the impact of media weak periodicity and the sensor sampling drift. The inter-frame period irregularity is often modeled as a Normal distribution, and has been proven successful in real media sensors [163]. In our study, we extend the past work, and prescribe the time that a sensory device outputs each media frame $t_{e}\left(f_{i, j}^{x_{0}}(k)\right)(\forall i, j, k)$ follow a Normal distribution with a shifted inter-frame period: $\mathbb{T}_{e}\left(f_{i, j}^{x_{0}}(k)\right) \sim \mathbb{N}\left(\mu_{i, j}^{x_{0}}(k), \sigma_{i}^{2}\right)$, where

$$
\mu_{i, j}^{x_{0}}(k)=(k-1) \cdot \Delta T_{i} \cdot \gamma_{i}+\text { offset }_{i, j}^{x_{0}}
$$

Here, $\mathbb{T}_{e}$ represents the random variable of $t_{e}$. The mean value in the distribution is decided by the average periodicity setting $\Delta T_{i}$ at the sender, the sensor sampling drift coefficient $\gamma_{i}$ (by referencing the global clock), and the time offset of the first media frame offset $x_{i, j}^{x_{0}}$ of the sensory stream $s_{i, j}^{x_{0}}$. The deviation is $\sigma_{i}$. We prescribe both $\Delta T_{i}$ and $\sigma_{i}$ are consistent for all sensory streams within any media modality $i$.

W.L.O.G, we assume for every media modality $i$ and its first intra-media SP $s p_{i}^{x_{0}}(1)$ includes media frames: $s p_{i}^{x_{0}}(1)=\left\{f_{i, 1}^{x_{0}}(1), f_{i, 2}^{x_{0}}(1), \ldots\right\}$. Hence, the assigned captured timestamp should satisfy:

$$
\forall i, j:\left|t_{c}^{*}\left(s p_{i}^{x_{0}}(1)\right)-\operatorname{offset}_{i, j}^{x_{0}}\right|<\Delta T_{i} / 2
$$

We also assume the first intra-bundle SP covers the set of intra-media SP: $s p^{x_{0}}(1)=\left\{s p_{1}^{x_{0}}(1), s p_{2}^{x_{0}}(1), \ldots\right\}$.

\section{- Accuracy efficiency}

Ideally, for each media modality $i$, the correct intra-media SP sequence is that the $\hat{k}$-th intra-media SP $s p_{i}^{x_{0}}(\hat{k})=\left\{f_{i, 1}^{x_{0}}(k), f_{i, 2}^{x_{0}}(k), \ldots\right\}$ for any integer $\hat{k}=k$. Due to the system constraints however, the

probability of a media frame $f_{i, j}^{x_{0}}(k)$ belonging to the intra-media SP $s p_{i}^{x_{0}}(\hat{k})$ for any integer $\hat{k}$ and $k$, in reality, only is:

$$
P\left(f_{i, j}^{x_{0}}(k) \in s p_{i}^{x_{0}}(\hat{k})\right)=\int_{t_{c}^{*}\left(s p_{i}^{x_{0}}(\hat{k})\right)-\frac{\Delta T_{i}}{2}}^{t_{c}^{*}\left(s p_{i}^{x_{0}}(\hat{k})\right)+\frac{\Delta T_{i}}{2}} \frac{1}{\sqrt{2 \pi} \sigma_{i}} e^{-\frac{\left(t-\mu_{i, j}^{x_{0}}(k)\right)^{2}}{2 \sigma_{i}^{2}}}
$$

When $\hat{k}=k$, Eqn. 3.21 returns the probability of correct intra-media SP identification. Otherwise, the frame $f_{i, j}^{x_{0}}(k)$ is identified with a wrong intra-media SP (i.e., $\hat{k} \neq k$ ) with the probability returned by Eqn. 3.21.

The intra-bundle SP, on the other hand, will not be directly affected by the variations of inter-frame period, due to the periodicity of time-realigned intra-media SP sequences. 


\section{- Time efficiency}

We claim that an intra-media SP sequence of the media modality $i$ needs to be resynchronized when any of its sensory streams has at least $C^{e r r}$ consecutive grouping errors (i.e., $f_{i, j}^{x_{0}}(k) \notin s p_{i}^{x_{0}}(\hat{k})$ when $\left.\hat{k}=k\right)$. For a single stream $s_{i, j}^{x_{0}}$, the probability of $C^{e r r}$ consecutive errors $P^{e r r}\left(s_{i, j}^{x_{0}}\right)$ is:

$$
P^{e r r}\left(s_{i, j}^{x_{0}}\right)=\prod_{c=0}^{C^{e r r}-1} P\left(f_{i, j}^{x_{0}}(k+c) \notin s p_{i}^{x_{0}}(\hat{k}+c)\right) \quad \text { when } \hat{k}=k
$$

where $c$ is the index shift variable. For analysis purpose, we compute for each sensory stream $j$,

$$
\left.k^{\mathrm{min}}\right|_{j}=\arg \min _{\left.k\right|_{j}}\left\{P^{e r r}\left(s_{i, j}^{x}\right)>P^{\mathrm{TH}}\right\}
$$

where $P^{\mathrm{TH}}$ is the lower bound of $P^{e r r}$. Hence, the actual resynchronization time is at $f_{i, j^{\min }}^{x_{0}}\left(\left.k^{\min }\right|_{j^{\min }}\right)$ such that:

$$
j^{\min }=\arg \min _{j}\left\{t_{c}\left(f_{i, j}^{x_{0}}\left(\left.k^{\min }\right|_{j}\right)\right\}\right.
$$

When a resynchronization of intra-media SP sequence is demanded, we reset all index numbers to one, and recompute the intra-media and intra-bundle SP sequences accordingly.

\subsubsection{Evaluation Results}

We use the real TEEVE testbed to evaluate the intra-media and intra-bundle SP identifications. Each sender site is configured with up to 4 video and 4 audio streams. We record the actual captured time $t$ of each multi-modal media frame at the corresponding specialized computation machines, synchronized by NTP. We only evaluate intra-media SP only, because the intra-bundle SP sequence is directly decided by the intramedia SP periodicity of different media modalities. To ease the description in this section, we omit the superscript $x$ of all mathematical denotations here, assuming all media data are captured at the same sender site.

Fig. 3.8 shows the intra-media SP identification results. In our setting, we use the inter-frame period $\Delta T_{V}=33.33 \mathrm{~ms}$ and $\Delta T_{A}=20 \mathrm{~ms}$. We evaluate, for each media frame, the difference of the assigned captured time and the actual captured time at the computation machines: i.e., $t_{c}-t_{e}$. Fig. 3.8(a), (c) present a snapshot $t_{c}-t_{e}$ of the video/audio frames, and (b),(d) show the overall distribution for each sensory stream. We find that most media frames result in a $t_{c}-t_{e}$ within $\Delta T_{V} / 2$ or $\Delta T_{A} / 2$, meaning that the captured time relabeling errors can be effectively minimized/constrained. Note that in Fig. 3.8(c), the two audio streams output curves with different slopes. The one with a steeper line indicates a microphone with a larger 
Fig (a)

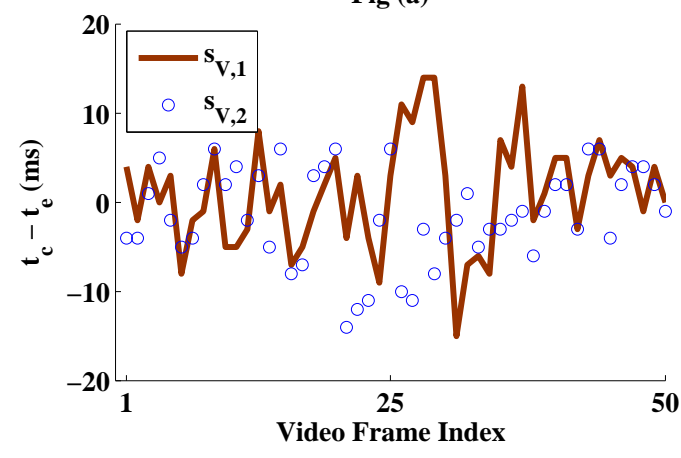

Fig (c)

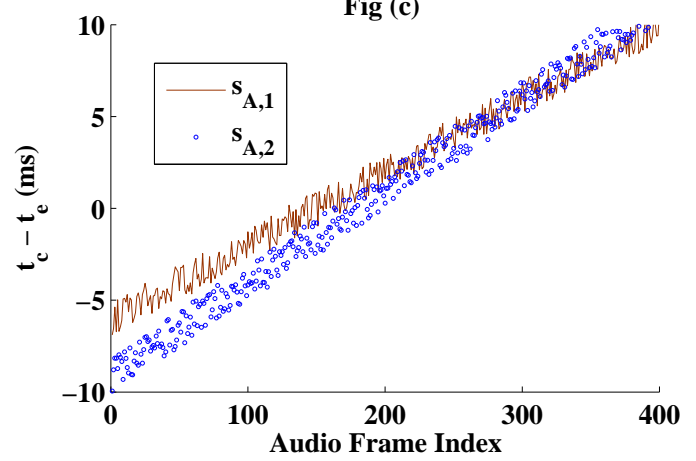

Fig (b)

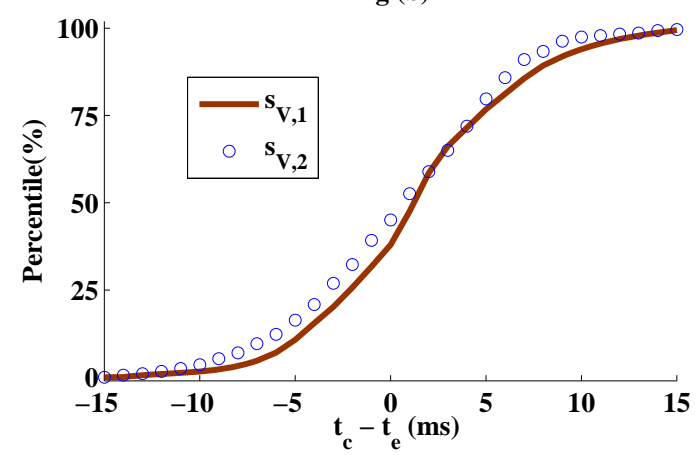

Fig (d)

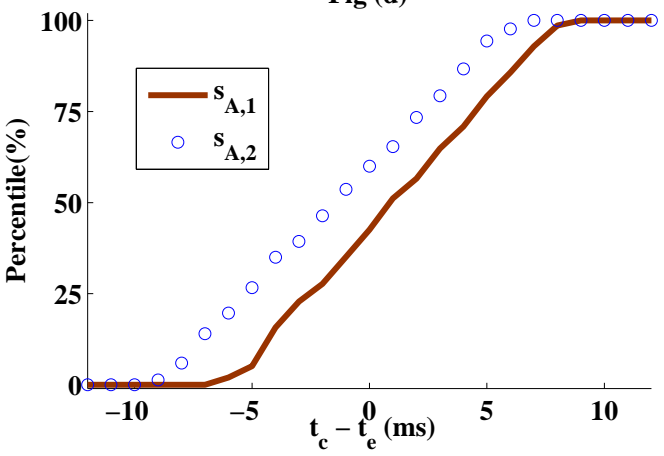

Figure 3.8: Distribution of $t_{c}-t_{e}$ for video and audio frames in the real teleimmersive testbed. (a) A snapshot of 50 video frames; (b) Distribution of all video frames; (c) A snapshot of 400 audio frames; (d) Distribution of all audio frames.

sampling drift. For an audio frame index greater than 400 in Fig. 3.8(c), the probability of being identified into a wrong intra-media SP will increase, because $t_{c}-t_{e}>\Delta T_{A} / 2$. In that case, a resynchronization is needed (time efficiency).

Fig. 3.9 shows the probability modeling results of the intra-media accuracy efficiency based on Eqn. 3.21. Fig. 3.9 (a),(b) evaluate the video intra-media SP with no drift $\left(\gamma_{V}=1\right)$. When $\gamma_{V}$ and $\Delta T_{V}$ are given, Eqn. 3.21 is mainly decided by two variables: the inter-frame period deviation $\sigma_{V}$, and $\beta_{V, j}=u_{V, j}(1)-$ $t_{c}^{*}\left(s p_{V}(1)\right)=\operatorname{offset}_{V, j}-t_{c}^{*}\left(s p_{V}(1)\right)$ (i.e., the time shift between the mean arrival time of the first media frame $f_{V, j}(1)$ in stream $s_{V, j}$ and the assigned timestamp of the corresponding intra-media SP $\left.t_{c}^{*}\left(s p_{V}(1)\right)\right)$. Hence, in Fig. 3.9 (a),(b), we present the probability of an accurate intra-media SP identification $\hat{k}=k$ of Eqn. 3.21) and a wrong (neighbor) identification (specifically, $\hat{k}=k+1$ ) at different $\sigma_{V}$ (the x-axis) and different $\beta_{V, j}$ (multiple curves in each figure). Generally, as $\sigma_{V}$ or $\beta_{V, j}$ increases, the probability of identifying a correct intra-media SP decreases, and the probability of being identified into the neighbor intra-media SP increases. Fig. 3.9 (c),(d) evaluate the impact of audio drift $\gamma_{A}>1$ on identifying the audio intra-media SP at each audio frame index of stream $s_{A, j}$. In both figures, we only consider $\beta_{A, j}=0$ (where $\beta_{A, j}$ is defined similar to $\left.\beta_{V, j}\right)$. We evaluate the probability of correct/wrong identifications at different $\left(\sigma_{A}, \gamma_{A}\right)$ pairs. As 

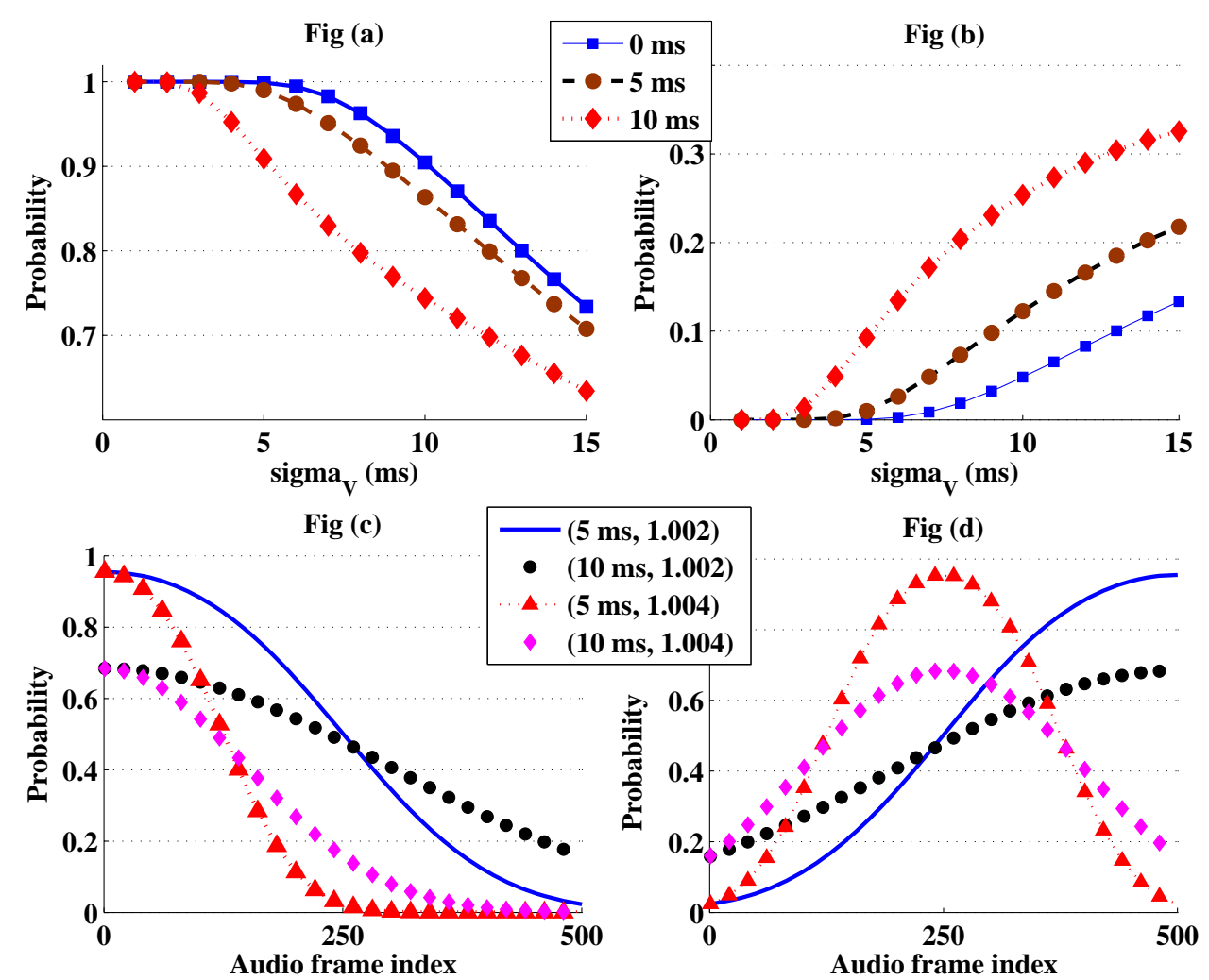

Fig (d)

$(10 \mathrm{~ms}, 1.002)$

$(5 \mathrm{~ms}, 1.004)$

(10 ms, 1.004)

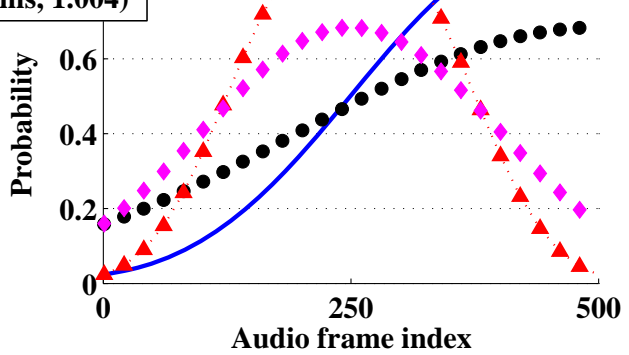

Figure 3.9: Fig.(a-b) show video intra-media SP identification with no drift. X-axis: $\sigma_{V}$. (a) Probability of a correct SP $(k=k)$, (b) Probability of a wrong SP $(k=k+1)$. The 3 curves in each figure: streams with different $\beta_{V, j}$ (i.e., $\beta_{V, j}=0,5,10 \mathrm{~ms}$ ). Fig.(c-d) show the impact of audio drift $\gamma_{A}>1$ on intra-media SP identification for stream $s_{A, 1}$ with $\beta_{A, 1}=0$. (c) Probability of a correct SP $(\hat{k}=k$ ), (d) Probability of a wrong SP $(\hat{k}=k+1)$. The 4 curves in each figure: different $\left(\sigma_{A}, \gamma_{A}\right)$ pairs.

the audio frame index enlarges, the sampling drift impact on the audio intra-media SP identification also increases. We find that in Fig. 3.9 (d), there is a peak in identifying an audio frame into a wrong (neighbor) SP $(\hat{k}=k+1)$. The reason is that as the audio index further increases without resynchronization, we could identify the audio frame into a wrong SP with $\hat{k}=(k+2),(k+3), \ldots$

\subsubsection{Remarks}

Here, we have three remarks in terms of the impacts on the synchronization controls and evaluations.

1. We have found that relabeling captured timestamp of multi-modal media frames using our algorithm has minimal impacts on the intra-stream synchronization during the media presentation, due to the bounded time relabeling errors.

2. The intra-bundle and intra-media SP sequences can explicitly tell the time correlations of media 
frames within each bundle. These correlations can be used to evaluate the intra-media and intrabundle synchronization skews in different locations.

3. The intra-session (inter-receiver or inter-sender) synchronization skews can be evaluated by comparing the latencies of multiple intra-bundle SP (from different sender sites, or to different receiver sites), assuming the global time availability.

4. The media data at each intra-bundle SP carries the most important synchronization information, so they should be given the highest priority in allocating bandwidth resources during the media distribution. In other words, each site may receive some sensory streams (usually those which are not synchronization references) from a sender with a reduced frame rate, where only the media frames within the intra-bundle SP are secured. This further proves the importance of evaluating the skews at the intra-bundle SP sequences.

In Section 4, we will present multi-tier collaborative synchronization controls based upon the above remarks.

\subsection{Summary}

In this section, we have presented a novel multi-dimensional synchronization model that takes into account the device/stream heterogeneity and scalability, system pipeline configurability, and multifunctional activity dependency, as well as a new synchronization point algorithm to identify the time correlations of multimodal media frames. Our work indicates a pioneering direction that the synchronization framework needs to be taken as our multimedia environments are getting richer in terms of multi-modal devices, more powerful in terms of computing power, and faster in terms of the high network inter-connectivity. These novel synchronization specifications will be employed and evaluated as an integral part of the overall synchronization framework throughout our study.

\subsection{Table of Abbreviations and Notations}

\section{List of Abbreviations in Chapter 3:}

NTP Network Time Protocol 
PTP Precision Time Protocol

SP Synchronization Point

SR Synchronization Region

W.L.O.G Without Loss of Generality

\section{List of Notations in Chapter 3:}

\begin{tabular}{|c|c|}
\hline$x$ & Index of sender site \\
\hline$i$ & Index of media modality \\
\hline$j$ & Index of sensory stream \\
\hline$k$ & Index of media frame \\
\hline$\left.k\right|_{j}$ & Index of media frame of stream $j$ \\
\hline$\hat{k}$ & Index of intra-media synchronization point (note it is $\hat{k}$ ) \\
\hline$\left.\hat{k}\right|_{i}$ & Index of intra-media synchronization point of media modality $i$ (note it is $\left.\hat{k}\right|_{i}$ ) \\
\hline$\hat{l}$ & Index of intra-bundle synchronization point (note it is $\hat{l}$ ) \\
\hline$n^{x}$ & Sender site $x$ \\
\hline$n^{y}$ & Receiver site $y$ \\
\hline$u^{x}$ & Media bundle from sender $n^{x}$ \\
\hline$r u^{x \rightarrow y}$ & Requested media bundle from sender $n^{x}$ to receiver $n^{y}$ \\
\hline$m_{i}^{x}$ & $i$-th media modality within $u^{x}$ \\
\hline$s_{i, j}^{x}$ & $j$-th sensory stream in media modality $m_{i}^{x}$ \\
\hline$f_{i, j}^{x}(k)$ & $k$-th media frame in $s_{i, j}^{x}$ \\
\hline$N$ & Number of all sites in the session \\
\hline$N_{A P}$ & Number of activity participants (senders) in the session \\
\hline$N U$ & Number of media bundles in the session \\
\hline$N M^{x}$ & Number of media modalities in bundle $u^{x}$ \\
\hline$N S_{i}^{x}$ & Number of sensory streams in $m_{i}^{x}$ \\
\hline$\Delta T_{i}$ & (Average) Inter-frame period of media modality $i$ \\
\hline$\Delta T_{u}$ & Period of intra-bundle synchronization point sequence \\
\hline
\end{tabular}




\begin{tabular}{|c|c|}
\hline$s p_{i}^{x}(\hat{k})$ & $\hat{k}$-th intra-media synchronization point of media modality $m_{i}^{x}$ \\
\hline$\left\{s p_{i}^{x}(\hat{k})\right\}$ & Periodic intra-media synchronization point sequence \\
\hline$s p^{x}(\hat{l})$ & $\hat{l}$-th intra-bundle synchronization point of media bundle $u^{x}$ \\
\hline$\left\{s p^{x}(\hat{l})\right\}$ & Periodic intra-bundle synchronization point sequence \\
\hline$n^{*}$ & Intra-session synchronization reference (reference site) \\
\hline$m_{*}^{x}$ & Intra-bundle synchronization reference (reference media modality) within $u^{x}$ \\
\hline$s_{i, *}^{x}$ & Intra-media synchronization reference (reference stream) within $m_{i}^{x}$ \\
\hline$f_{i, j}^{x}(*)$ & Intra-stream synchronization reference (reference frame) within $s_{i, j}^{x}$ \\
\hline$D$ & Any general latency, e.g., $D_{r g}, D_{r l}, D_{i s}$ \\
\hline$\Delta D\left(f_{i, j}^{x}(k), n^{y}\right)$ & ) Intra-stream synchronization skew of $f_{i, j}^{x}(k)$ when distributed to receiver $n^{y}$ \\
\hline$\Delta D\left(s_{i, j}^{x}, n^{y}\right)$ & Intra-media synchronization skew of $s_{i, j}^{x}$ when distributed to receiver $n^{y}$ \\
\hline$\Delta D\left(m_{i}^{x}, n^{y}\right)$ & Intra-bundle synchronization skew of $m_{i}^{x}$ when distributed to receiver $n^{y}$ \\
\hline$\Delta D\left(u^{x}, n^{y}\right)$ & Intra-session synchronization skew of $u^{x}$ when distributed to receiver $n^{y}$ \\
\hline$t_{c}$ & Assigned captured time at the media sensor \\
\hline$t_{e}$ & Actual arrival time at the encoding machine \\
\hline$t_{e}^{\prime}$ & Realigned periodic arrival time at the encoding machine \\
\hline$t^{\text {left }}$ & Left bound (start time) of an intra-media synchronization region \\
\hline$t^{\text {right }}$ & Right bound (end time) of an intra-media synchronization region \\
\hline $\operatorname{offset}_{i, j}^{x}$ & Time offset of the first media frame $f_{i, j}^{x}(1)$ of $s_{i, j}^{x}$ \\
\hline $\mathbb{T}_{e}$ & Distribution (random variable) of $t_{e}$ \\
\hline$\mu_{i, j}^{x}(k)$ & Mean arrival time of $f_{i, j}^{x}(k)$ \\
\hline$\sigma_{i}$ & Standard deviation of media modality $i$ \\
\hline$\gamma_{i}$ & Sampling drift of media modality $i$ \\
\hline$P$ & Probability \\
\hline$P^{e r r}$ & Error probability \\
\hline$C^{e r r}$ & Consecutive error number of media frames \\
\hline$P^{\mathrm{TH}}$ & Error probability lower bound \\
\hline$\beta_{i, j}$ & $\beta_{i, j}=\operatorname{offset}_{i, j}^{x}-t_{c}\left(s p_{i}(1)\right)$ \\
\hline $\mathrm{CF}$ & Contribution factor \\
\hline$\vec{O}\left(s^{x} i, j\right)$ & Orientation of stream $s^{x} i, j$ \\
\hline$\vec{O}(x, y)$ & Desired receiver $n^{y}$ 's view orientation from $n^{x}$ 's streams \\
\hline
\end{tabular}




\section{CHAPTER 4}

\section{DESIGN OF MULTI-TIER SYNCHRONIZATION CONTROLS}

The multi-layer (intra-stream, intra-media, intra-bundle and intra-session) synchronization skews can be introduced in all tiers (locations) in teleimmersion. Without appropriate controls in each tier, these skews can be propagated, and the accumulated effect will impose difficulties on final synchronous presentation of multimedia data at the receiver output devices. Hence, we investigate a multi-tier collaborative synchronization control framework, where multi-layer synchronization skews are constrained in all three tiers. Our study in this chapter is non-perception based, meaning that the synchronization control adaptations are independent of real human perceptual feelings in heterogeneous teleimmersive activities.

\subsection{Overview}

The multi-tier collaborative synchronization control framework includes the following key components:

- Capturing Tier. We develop CloudStream, a cloud-based media processing/encoding parallelization and scheduling scheme for computation-intensive media like 3D multiview videos. Our cloud scheduler is able to predict the computation demand of each media frame, and decide the minimal amounts of cloud resources, in order for speeding up the computing time and smoothing the computing jitter. This allows the teleimmersive systems to meet the synchronization demands at the capturing tier.

- Distribution Tier. We propose SyncCast, a synchronized multicast overlay for multi-modal multistream distribution in teleimmersion. Our overlay topology is able to find the shortest distribution paths for each sensory stream under the multi-layer synchronization constraints. Its design is based on adapting the bandwidth allocations based on the contributions of media data to end user interests, for the purpose of maximizing the bandwidth utilization to send most important media data to all participating sites within each teleimmersive session. A cooperative frame rate allocation scheme is employed to address the issue of bandwidth insufficiency in SyncCast. 


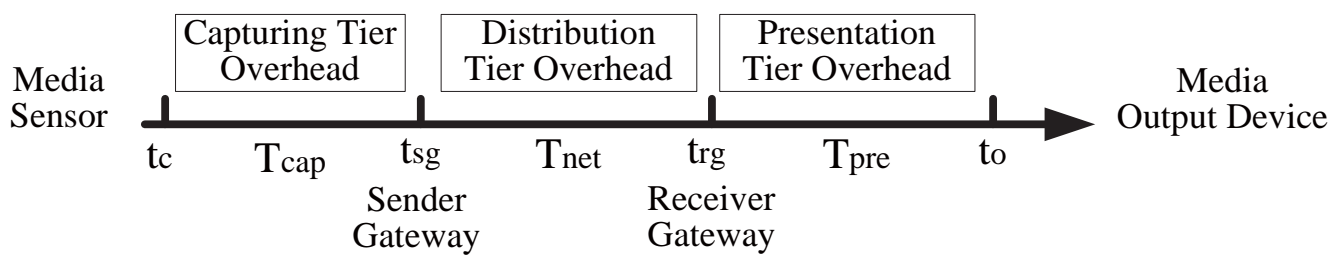

Figure 4.1: Multi-tier synchronization control timeline.

- Presentation Tier. We discuss TBuffer, an adaptive presentation scheduling scheme which is able to perform, under Internet jitter degradations, final in-sync multi-modal media presentation at distributed receiver sites, while satisfying the demands of media signal intelligibility at the best interactive quality.

Fig. 4.1 shows the timeline of multi-tier synchronization controls, where $t_{c}$ is the media capturing time (derived from Section 3.3), $t_{o}$ represents the presentation time at the receiver output devices, $t_{s g}$ and $t_{r g}$ denote the arrival time of media data at the sender and receiver gateways. Hence, our multi-tier synchronization control framework achieves multimedia synchronization in each tier by adapting $T_{\text {cap }}, T_{n e t}$ and $T_{\text {pre }}$ respectively. Throughout the chapter, we follow the same mathematical denotations to describe the multimedia data as in Section 3.1.

\subsection{CloudStream: Capturing Tier Control}

The goal of CloudStream is to employ the minimal (computation) cost to achieve the multimedia synchronization at each sender site. The intra-bundle skews can be reduced by outsourcing the media computation tasks to the cloud infrastructure to speed up the processing/encoding process of computation-intensive media like 3D multiview videos. The multi-layer synchronization constraints can be further realized by deciding the amounts of cloud resources based on predicted media computation demands in order to smooth the resulting computation jitter. Due to the negligible computation overhead on audios, haptics and etc, we only focus on the parallelization of 3D multiview videos.

An illustration of the CloudStream setup is presented in Fig. 4.2. Note that for video streams targeting for active receivers, the communication latency between the TEEVE testbed and the cloud must be much smaller than the actual media computation time, for the sake of interactive quality. In this case, we may employ the campus cloud infrastructure that are colocated in the same LAN as the TEEVE testbed. For passive receivers where the interactive quality is less important, commercial cloud infrastructures with a reasonable communication latency can be used. 


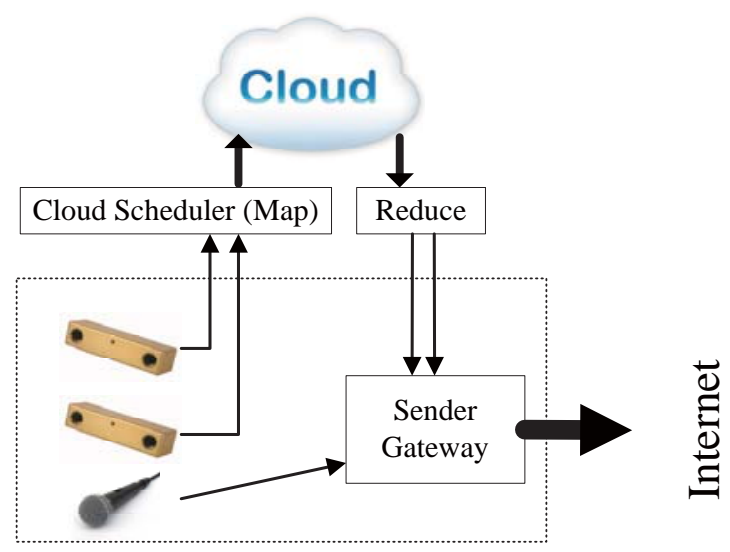

Figure 4.2: An illustration of CloudStream setup.

\subsubsection{Design of CloudStream}

\section{- Design Rationale for Multi-level Parallelization}

We propose our parallelization scheme. Previous studies have achieved the video encoding parallelization on a single multi-core computer with and without GPU support $[25,26]$, or in a cloud infrastructure like Google Hangout [165], but none of them provides the real-time synchronization support.

$3 D$ multiview video coding structures. We employ the 3D multiview video codec developed in UC Berkeley [166]. A 3D multiview image carries a set of time-correlated 3D singleview frames (i.e., an intramedia synchronization point as in Section 3.3), and the sequence of singleview frames from the same view is processed and encoded in an individual video stream (i.e., different streams represent different views). There is no coding dependency across multiple views belonging to the same multiview image, and across the singleview frames within each stream. When encoding a singleview frame, the codec has an internal support for data parallelization using OpenMP [167]. Several encoding memory spaces need to be shared across different data partitions.

Inter-node and intra-node parallelism. To exploit the compute nodes returned by the cloud at full potential, we need to parallelize the encoding scheme across different compute nodes (inter-node parallelism) which do not share the memory space. On the other hand, the shared-memory address space of the parallelism inside one compute node (intra-node parallelism) will ease the management of sharing information or states with the help of threads synchronization by locks or barriers. However, thread synchronization can cause overhead and may serialize the whole encoding, offsetting the performance gains from parallelization. Additionally, these two different types of parallelism prefer different work granularity. As a rule of thumb, the inter-node parallelism has much larger granularity than its counterpart. 


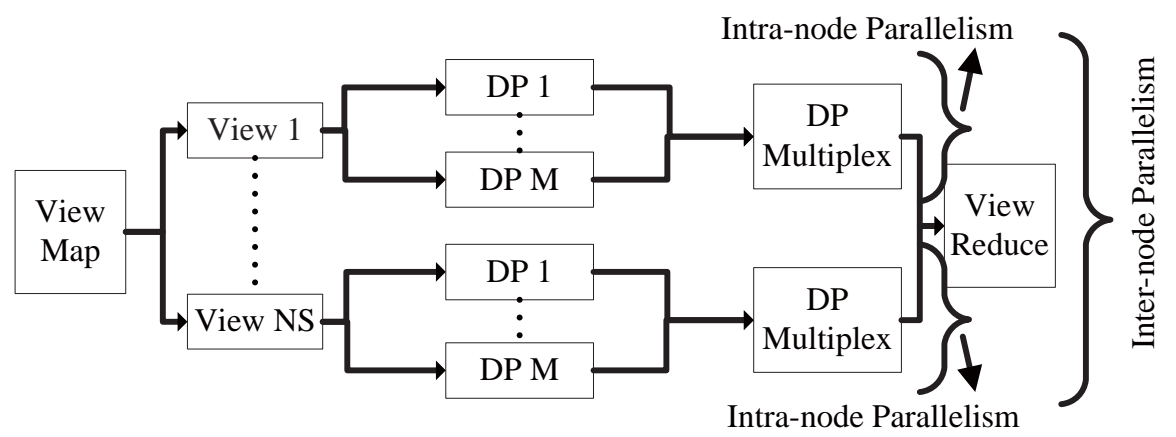

Figure 4.3: Multi-level parallelization in the cloud. DP: data partition within 3D singleview video frame. $N S$ : number of views (video streams), $M$ : number of DP within each stream.

Choice of our parallelization scheme. From the above discussions above, we propose our multi-level encoding parallelization scheme. The idea of our scheme is shown in Fig. 4.3. In our scheme, since the coding-independent multiview images have the largest work granularity, encoding at the view level is an ideal candidate to be parallelized across different compute nodes. The internal parallelization support for encoding singleview frames makes it ideal for intra-node parallelism, which can be easily implemented using OpenMP by encoding each slice on a different CPU within a compute node (Fig. 4.3).

\section{- Prediction of Computation Demand}

We predict the single-core computation time for 3D singleview frames within each video stream for any sender site $n^{x}$. Given the $k$-th frame $f_{\mathrm{V}, j}^{x}(k)$ of the $j$-th video streams, its predicted computation demand $\tilde{T}_{c a p}\left(f_{\mathrm{V}, j}^{x}(k)\right)$ can be computed using a linear predictor:

$$
\tilde{T}_{c a p}\left(f_{\mathrm{V}, j}^{x}(k)\right)=\sum_{l=1}^{L} a_{l} \times T_{c a p}\left(f_{\mathrm{V}, j}^{x}(k-l)\right)
$$

where $T_{\text {cap }}$ represents the actual single-core computation time, $L$ is the predictor order and $q(l=0,1, \ldots, L)$ are the coefficients. In our study, these coefficients $\left\{a_{l}\right\}$ are computed offline using the Levinson-Durbin recursion approach. We have tested different order $L$, and found that any $L \in[610]$ can achieve a prediction accuracy of $90 \%$ of samples within 5\% deviation, and $99 \%$ of samples within $10 \%$ deviation. The accuracy can be further improved by offline profiling $[168,169]$, whose details are not the focuses of this study.

\section{- Inter-node Parallelism}

For inter-node parallelism, the cloud scheduler simply maps a newly arrived singleview video frame to a compute node (usually realized by a virtual machine). However, different compute nodes can be configured with unequal number of CPUs. Our CloudStream scheme prefers the compute node with the minimal cost 

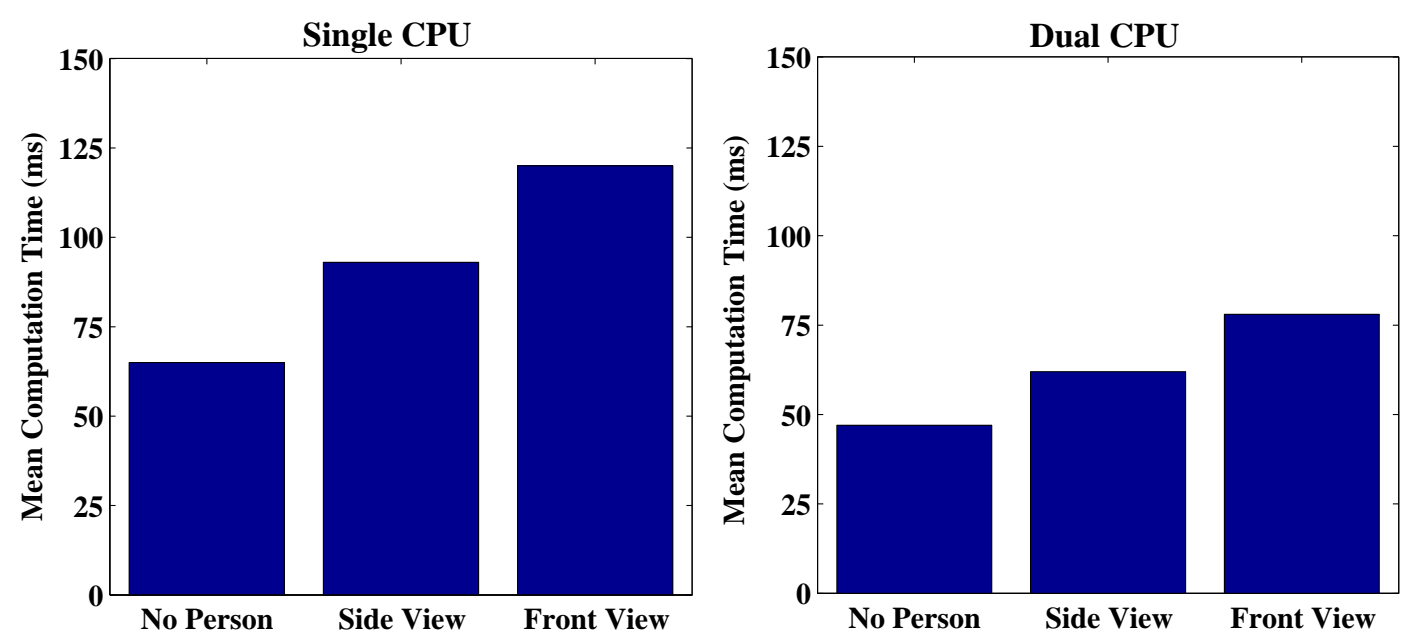

Figure 4.4: Average computation time of video frames using single or dual CPU for under three scenarios: no person in the scene, side view, and front view.

(i.e., the number of CPUs) that meets the synchronization demand at the capturing tier, as discussed below.

\section{- Intra-node Parallelism}

We realize the multi-layer synchronization demand by bounding the predicted multi-core computation time of the $3 \mathrm{D}$ video frames within a preset interval $T_{c a p}^{\mathrm{H}}$, i.e,

$$
\forall x, y, j: \tilde{T}_{c a p}\left(f_{\mathrm{V}, j}^{x}(k), n^{y}\right)<T_{c a p}^{\mathrm{H}}
$$

where $\tilde{T}_{c a p}\left(f_{\mathrm{V}, j}^{x}(k), n^{y}\right)$ is the predicted computation time of the video frame $f_{\mathrm{V}, j}^{x}(k)$, which is equal for all receiver sites $n^{y}$ in our system.

Given the predicted single-core computation time $\tilde{T}_{c a p}$ for each media frame $f_{\mathrm{V}, j}(k)$, and the computation demand for the sequential encoding part in the codec $\Delta T_{s e q}$, the minimal number of CPU required to achieve the synchronization is:

$$
M^{\min }\left(f_{\mathrm{V}, j}^{x}(k)\right)=\left\lceil\frac{\tilde{T}_{c a p}\left(f_{\mathrm{V}, j}^{x}(k)\right)-\Delta T_{s e q}}{T_{c a p}^{\mathrm{H}}-\Delta T_{s e q}}\right\rceil
$$

\subsubsection{Evaluation Results}

In our cloud testbed, we use up to four CPUs (Intel Xeon 2.8 GHz) for each compute node. Fig. 4.4 shows the average computation time of video frames using either single or dual CPU under three scenarios: (1) no person in the scene, (2) a side view of person, and (3) a front view of the person. Because these scenarios carry heterogeneous amounts of visual information, the corresponding computation demand is also different. 

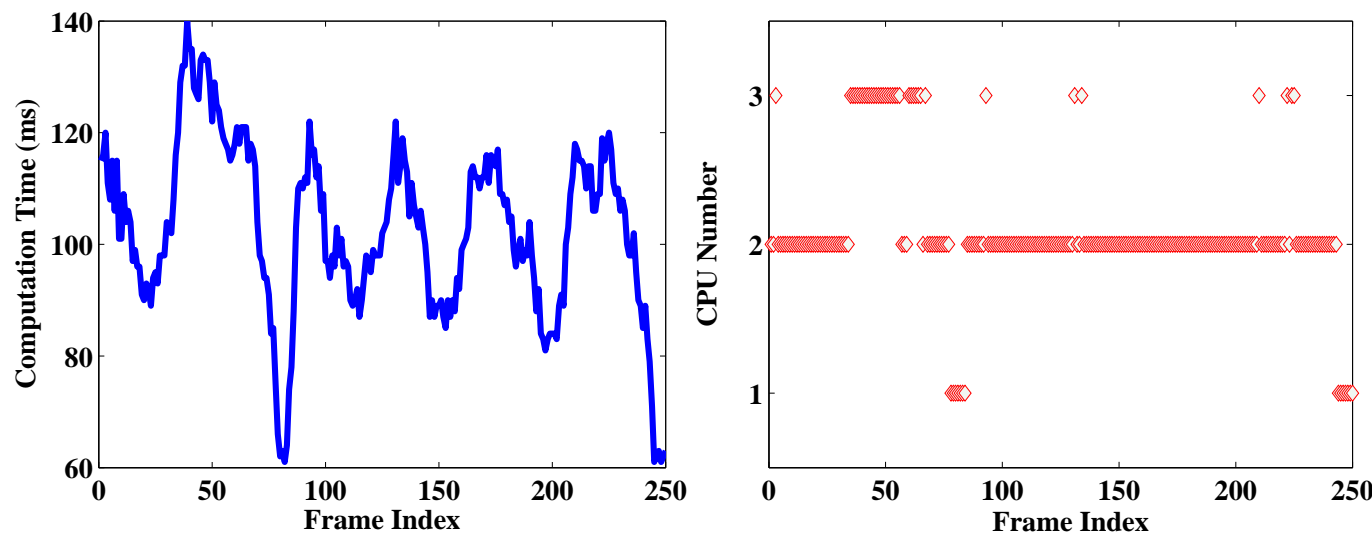

Figure 4.5: Left figure shows the computation time for each video frame using single CPU. Right figure depicts the minimal number of CPU required to achieve synchronization constraints when $T_{c a p}^{\mathrm{H}}=75 \mathrm{~ms}$.

In general, a greater amount of visual information can demand a larger computation time. We also use the Amdahl's Law to infer the sequential processing part of the video codec, and we find $\Delta T_{\text {seq }} \approx 30-35 \mathrm{~ms}$.

Fig. 4.5 shows the variations of computation demand for each video frame using single CPU. Based on the estimated $\Delta T_{\text {seq }}$, we compute the resulting minimal CPU number required to achieve synchronization constraints under $T_{c a p}^{\mathrm{H}}=75 \mathrm{~ms}$. The majority of video frames require two CPUs to achieve the computation time below $T_{c a p}^{\mathrm{H}}$. As a rule of thumb, the scenario with no person only requires only one CPU because very few visual data are carried in the black background, while the scenario with a front view of a person demands more than two CPUs owning to the more complicated visual contents.

\subsection{SyncCast: Distribution Tier Control}

The distribution of bandwidth-consuming 3D multiview videos is a problem because the availability of the network bandwidth resources cannot match the growing need of the multiview camera sensors. This means that not all video streams can be supported during the multicast. In teleimmersion, our SyncCast overlay gives priority to the distribution of video streams that are most important to end user interests (based on Eqn. 3.1). We will assume that the audio, haptic, body sensory and many other streams (except video) are guaranteed distribution, due to their negligible data demand.

We present the design of the SyncCast, a multicast scheme which is able to maximize the bandwidth utilization to distribute multi-modal multi-streams sourced at multiple teleimmersive sender sites with the minimal distribution latencies. We will focus on the distribution of the videos. To deal with the bandwidth insufficiency issues, a cooperative video frame rate allocation algorithm is also included in SyncCast. Our 


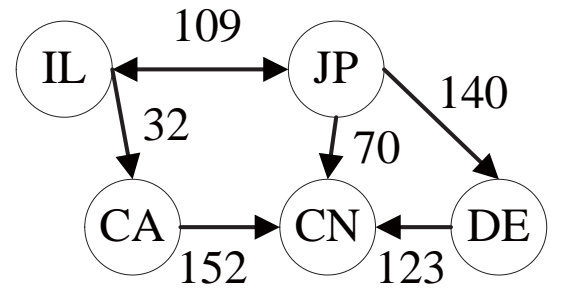

(a)

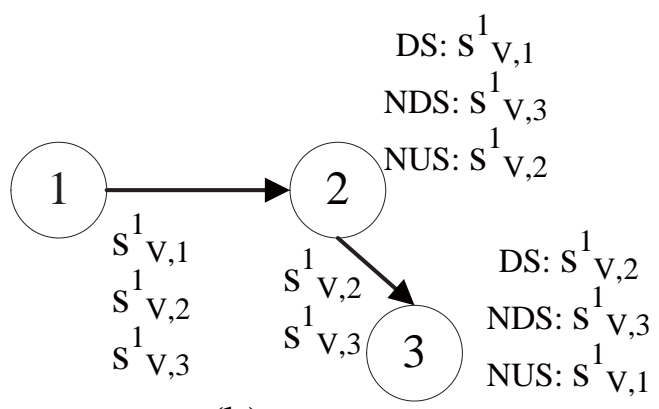

(b)

Figure 4.6: (a) An example of different path options (numbers are indicated in ms). (b) An example of video dominance and path prioritization

SyncCast is based upon the previous teleimmersive multicast study called ViewCast [12], which did not investigate the synchronization impact in building the overlay. We will compare our design with ViewCast throughout the section.

\subsubsection{System Model}

\section{- Topology Model}

We present the topology model based on the media data model described in Section 3.1. In multi-site teleimmersion, each of the $N$ sites can be classified as a sender site or/and a receiver site. A sender site can itself also be a receiver. We let $\mathcal{E}^{s n d}$ denote the set of sender sites in the system, where $N_{A P}=\left|\mathcal{E}^{s n d}\right|$. In our study, we assume that for each sender site $n^{x} \in \mathcal{E}^{s n d}$, all other $N-1$ sites within the session are the receiver sites of $n^{x}$.

We use $L^{x, x^{\prime}}$ to describe the link cost between two neighboring sites $n^{x}$ and $n^{x^{\prime}}$, and its cost is $\mathcal{C}\left(L^{x, x^{\prime}}\right)$. For example, in Fig. 4.6(a), the link cost between site IL and site JP is 109 ms. Note that there may not be a direct link between two sites due to the potential connectivity problem in the real world. A path from a sender site $n^{x}$ to a receiver site $n^{y}$ may be routed through several intermediate sites in the overlay, and there can be multiple path options between the sender and receiver. We denote the set of path options as $\mathcal{P}^{x, y}$. To simplify the problem, we assume $\mathcal{P}^{x, y}$ is a sorted list, where the $k$-th shortest path is $\mathcal{P}^{x, y}[k]$, and its cost is $\mathcal{C}\left(\mathcal{P}^{x, y}[k]\right)$. The path cost can be computed by summing up all the link costs on the path and the transmission latencies ${ }^{1}$ incurred on sender, intermediate and receiver sites. We assume in our study that these transmission latencies are negligible. For example in Fig. 4.6(a), we have three paths between IL and

\footnotetext{
${ }^{1}$ The transmission latency is the delay incurred that are used to push byte streams from the network socket to the physical wireline/wireless links.
} 
CN with different costs: 184 (via CA), 179 (via JP), and 372 (via JP and DE).

Each site $n^{x}$ has an in-degree and out-degree bandwidth upper bound (i.e., $\max B_{\text {in }}^{x}$ and $\max B_{\text {out }}^{x}$ ) to constrain the bandwidth usage $B_{\text {in }}^{x}$ and $B_{\text {out }}^{x}$. To simply the problem, the unit of all bandwidth-related metrics is represented in number of video streams in our study. We assume the bandwidth consumptions of all other media modalities are negligible compared to the 3D videos.

\section{- Visual Contributions of Video Streams}

Here, we extend the concept of the visual contribution factor (CF) proposed in Section 3.2, and prioritize the video streams into three categories at each receiver site.

- Dominant Stream (DS). A video DS is the stream with the maximum CF (i.e., the synchronization reference video stream) among all other same-source video streams from each sender. A receiver site can have a set of video DS from different senders (one DS from each sender). The quality of the video DS directly decides the satisfaction of user interests. For example in Fig. 4.6(b), the sender site 1 outputs three video streams, where $s_{\mathrm{V}, 1}^{1}$ is the DS of receiver site 2 , and $s_{\mathrm{V}, 2}^{1}$ is the DS of receiver site 3.

- Non-Dominant Stream (NDS). The set of video NDS are the streams with a positive CF (CF $>0)$ from each sender. A video NDS has partial visual contributions to the target receiver. For example in Fig. 4.6(b), $s_{\mathrm{V}, 3}^{1}$ is the video NDS for both receiver sites 2 and 3.

- Non-Use Stream (NUS). The set of video NUS are the streams with a non-positive $\mathrm{CF}(\mathrm{CF} \leq 0)$ from each sender. A video NUS has no contribution to the receiver. For example in Fig. 4.6(c), $s_{V, 2}^{1}$ is the NUS of receiver site 2, and $s_{\mathrm{V}, 1}^{1}$ is the NUS of receiver site 3 .

A receiver site only requests video DS and NDS from each sender. Note that a video stream can serve as a DS for some receivers, while as a NDS or NUS for others within the whole multi-site teleimmersive session. To describe the visual contributions of a video streams to all receiver sites within the system, we further propose the metric Global Contribution Factor (GCF) as follows:

$$
\operatorname{GCF}\left(s_{V, j}^{x}\right)=\sum_{y} \vec{O}\left(s_{V, j}^{x}\right) \cdot \vec{O}^{x, y}
$$

Here, both $\vec{O}\left(s_{V, j}^{x}\right)$ and $\vec{O}^{x, y}$ are defined in Section 3.2. A video stream with a larger GCF is visually more important to whole system than other streams with smaller GCF. 
In comparison, ViewCast does not prioritize video streams based on GCF, but rather solely on the CF of video streams to each receiver site. This can create an issue when two video streams are competing for the limited bandwidth resources (one with a small CF to one specific receiver and a large GCF, and the other with a large $\mathrm{CF}$ to the same receiver and a small GCF). ViewCast in this case will prefer the second stream. As an insufficient bandwidth availability, ViewCast's policy may lead to the dropping of the video stream with the larger GCF, which can potentially affect numerous receiver sites demanding the stream as the video DS. This leads to the concept of victims as discussed below.

\section{- Victim Sites and Victim Streams}

We propose two victim concepts to describe the cases when the receiver sites do not receiver all video streams.

Definition 4.3.1 A victim site is a receiver site which cannot successfully receive video DS from all sender sites. In other word, there can be at least one video DS (from one sender) missing at a victim site.

Definition 4.3.2 A victim stream is a video DS or NDS stream (from any sender site) which is not successfully received by a receiver site.

In comparison, ViewCast evaluates its performance by solely counting the number of victim streams.

\subsubsection{Problem Formulation}

Based on the prioritization of video streams and the concept of victims, we formulate the problem of SyncCast. We will focus on distributing the video streams in SyncCast under both multi-layer synchronization constraints and bandwidth constraints. In order to maximize end user interests with the best interactive quality and minimize the number of victim sites, we prescribe that all video DS within the multi-site teleimmersive session should be given the highest priority in allocating the bandwidth resources and finding the shortest paths. We then minimize the number of victim streams rate by distributing video NDS with the best bandwidth utilization.

We simplify the distribution tier problem by assuming that all other media data (like audios, haptics and etc.) between each sender and receiver sites follow the same distribution paths as the corresponding video DS. This will allow us to guarantee no intra-bundle skew and no intra-media skew for media modalities except video. We also leave the intra-stream synchronization issue to the presentation tier control. Hence, 
the intra-media skews of the videos and intra-session (both inter-sender and inter-receiver skews) are the main synchronization concerns in SyncCast.

We denote the average latency of media data incurred during the distribution as $T_{\text {net }}$. For example, we can use $T_{n e t}\left(s_{i, j}^{x}, n^{y}\right)$ to represent the average latency of the stream $s_{i, j}^{x}$ when it is distributed to $n^{y}$. In addition, because all other media modalities follow the same distribution path as the video reference stream (i.e., the video DS) between a sender and a receiver, we get $T_{n e t}\left(u^{x}, n^{y}\right)=T_{n e t}\left(m_{i}^{x}, n^{y}\right)=T_{n e t}\left(s_{i, *}^{x}, n^{y}\right)$. We also use $\zeta_{1}$ and $\zeta_{2}$ to denote the number of victim sites and victim streams. The upper bounds of synchronization skews are prescribed as $\delta_{1}(i)$ for the intra-media layer, $\delta_{2}(i, *)$ for intra-bundle layer, and $\delta_{3}$ for intra-session layer. Here, $\delta_{1}(i)$ value varies at different media modality $i$, while $\delta_{2}(i, *)$ value is decided by the media modality $i$ and the reference modality $*$. Hence, in the distribution tier, $\delta(i, *)=0$ and $\delta_{1}(i)=0\left(i \neq{ }^{\prime} \mathrm{V}^{\prime}\right)$. $\delta_{1}(\mathrm{~V})$ and $\delta_{3}$ are preset constant positive numbers. Both inbound and outbound bandwidth usage should also be bounded. The overall problem can be formulated in the following general form:

- Minimizing victim sites and victim streams:

$$
\text { MIN } \zeta_{1} \text { and } \zeta_{2}
$$

- Minimizing average system EED of video DS paths:

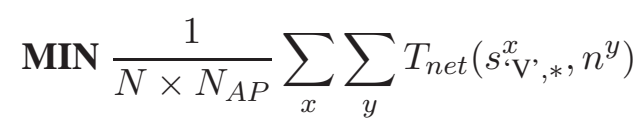

- Synchronization constraints $(\forall x, y, i, j)$ :

$$
\begin{aligned}
\left|\Delta T_{n e t}\left(s_{i, j}^{x}, n^{y}\right)\right| & \leq \delta_{1}(i) \\
\left|\Delta T_{n e t}\left(m_{i}^{x}, n^{y}\right)\right| & \leq \delta_{2}(i, *) \\
\left|\Delta T_{n e t}\left(u^{x}, n^{y}\right)\right| & \leq \delta_{3}
\end{aligned}
$$

- Bandwidth constraint $(\forall x)$ :

$$
\forall n^{x}: B_{\text {in }}^{x} \leq \max B_{\text {in }}^{x}, \quad B_{\text {out }}^{x} \leq \max B_{\text {out }}^{x}
$$

\subsubsection{Path Selection Policies}

Multiple path options can be selected in the overlay, and different options can lead to diverse bandwidth utilization outcomes. So before the discussions of SyncCast design, we present our path selection policies 
with a goal to maximize the utilization of bandwidth resources in teleimmersion. We will first propose the metric fairness (or unfairness), which is closely related the bandwidth utilization.

\section{- Fairness of Path Options}

Due to the diversity of user views, different receivers may request different video DS and NDS. Previous studies $[27,28,29,30]$ assume the multi-stream homogeneity from the same sender, and hence, it is possible that some intermediate sites may have to relay some video streams which have no contribution (i.e., NUS) to themselves. For example in Fig. 4.6(b), site 2 has to relay $s_{\mathrm{V}, 2}^{1}$ even though it does not request the stream. This will cause an unfairness in the multicast overlay, which would otherwise waste the network bandwidth resources at these intermediate sites. A good scheme should be able to achieve fair routing in which the intermediate sites only relay streams that they themselves may request. To evaluate the fairness of different path options between the sender site $n^{x}$ and the receiver $n^{y}$ for a video stream $s$ captured at $n^{x}$, we compute on each path $\mathcal{P}^{x, y}[k]$, the number of intermediate sites where $s$ has no contribution (i.e., $s$ is a NUS to the intermediate site), and denote it as $\mathcal{Q}_{s}^{x, y}[k]$. We call it the unfairness value. For example in Fig. 4.6(b), the path from site 1 to site 3 via site 2 leads to an unfairness value equal to 1 for the video stream $s_{V, 1}$.

\section{- Heuristic Path Selection Policies}

To effectively preserve the bandwidth resources during the video multicast, we propose the following three path selection policies for multi-site teleimmersion.

- To improve the fairness of the distribution overlay while preserving the interactivity in the multi-site teleimmersion, we prioritize multiple path candidates based on a fairness-first, cost-next policy in our system, meaning that the paths with a smaller unfairness value are always placed at a higher priority, and that the path with a shorter cost (latency) is better than those with the same unfairness value but a longer cost (latency).

- No sender site is responsible for relaying its NDS sourced at all other senders.

- No site is responsible for relaying its NUS (which can be DS/NDS to other receivers) sourced at all senders.

By contrast, ViewCast preserves the bandwidth by guaranteeing the strict fairness: the receiver sites only request a video stream from either the sender site or those that have already requested and received the 


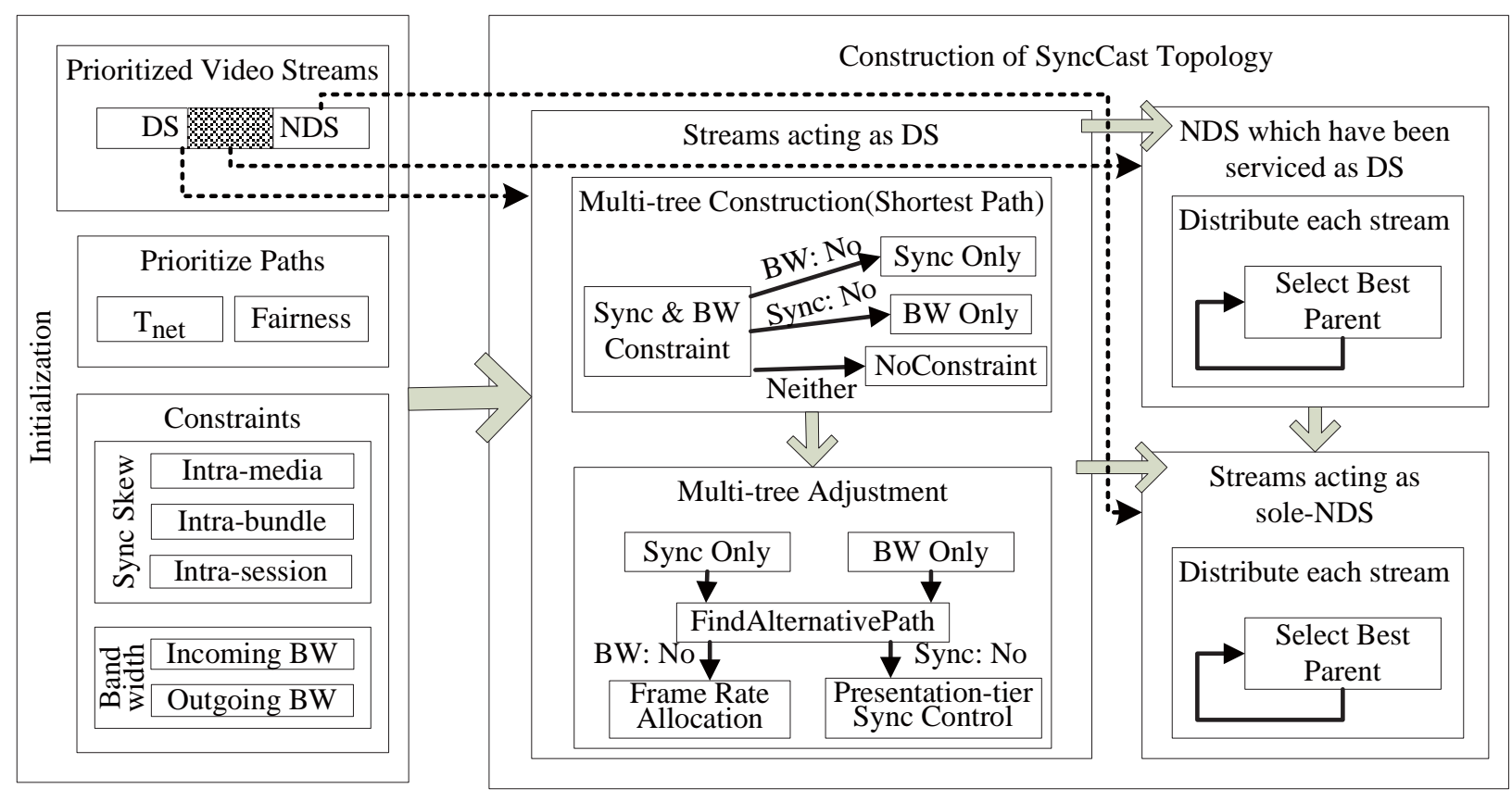

Figure 4.7: An overview of SyncCast.

stream. However, multiple video DS can compete for bandwidth resources at different sites. Without relaxing the fairness constraint, we may not guarantee the successful distribution of video streams (especially DS) in ViewCast.

\subsubsection{Design of SyncCast}

Based on the above discussions, we propose SyncCast design. The multicast overlay problems have been proven NP hard [12]. Hence, we use a heuristic approach to solve the problem. Fig. 4.7 shows an outline of the overall scheme.

\section{- An Overview}

The general idea is that SyncCast decides a multicast tree topology for each video stream (from a sender site to all receiver sites) within the whole session. However, due to heterogeneous visual contributions of video streams to different receiver sites, SyncCast finds the distribution paths for video DS and NDS in the order of the following three service classes. The order of video streams being serviced in each service class is decided by their GCF. In other words, the video streams with larger GCF are serviced earlier than those with smaller GCF in the same class.

SyncCast first determines the multicast trees for all video DS (class 1). The algorithm can be divided 
into two parts: multi-tree construction and multi-tree adjustment. During the stage of the multi-tree construction, SyncCast tries to find the latency/fairness prioritized path that satisfies both synchronization and bandwidth constraints. If there is no successful path, either bandwidth or the synchronization constraint has to be relaxed. During the stage of the multi-tree adjustment, SyncCast seeks alternative paths for previous successfully assigned paths, for the purpose of reducing the synchronization skews and/or the bandwidth overhead within the bounded allowance. After the two stages, SyncCast either achieves cooperative frame rate allocation at each site if the bandwidth constraints are still not satisfied, and/or leave the problem to the presentation tier (Section 4.5) if the synchronization constraints are still violated.

Next, SyncCast decides all video NDS which have been requested as DS by other receivers (class 2). In order to reduce the bandwidth overhead at the original sender sites, an option is to let these NDS receivers only request from the sites that have already received the stream (either as DS or as NDS). For each video stream, SyncCast determines all serviced sites (i.e., sites that received the stream) in the distribution topology before it picks the best parent site for an unserviced receiver (i.e. a site that has not received the stream).

Last, SyncCast finds possible distribution paths for all sole-NDS (class 3), i.e., video streams that have never been requested as DS. We will follow the same approach as in class 2 to pick the best parent sites.

To minimize the number of victim streams (which are usually bottlenecked at the sender sites due to the inadequate bandwidth availability), we do not request a video NDS directly from the original sender site, unless it has never be serviced (i.e., the stream has never been distributed to any receiver site).

By contrast, ViewCast only sets an upper bound for $T_{n e t}$ without further taking any further action on the synchronization constraints. The algorithm simply picks a site with the least bandwidth overhead among the sender and the sites which have already received the stream. Hence, multiple streams from a same sender to a same receiver can follow paths with huge $T_{n e t}$ differences. This will cause noticeable intra-media skew among the video streams. The intra-session skews, on the other hand, can only be constrained within the $T_{n e t}$ upper bound.

\section{- Distribution of Video DS in Class 1}

During the stage of the multi-tree construction, SyncCast finds, between each sender and receiver, the best prioritized path that satisfies both bandwidth and intra-session synchronization constraints. We call a path successful if both constraints can be addressed. Otherwise, SyncCast considers three cases:

1. If there are paths satisfying only the intra-session synchronization constraints, SyncCast selects, 
among these paths, the one with the least number of intermediate sites hat violate the bandwidth upper bound.

2. If there are paths satisfying only the bandwidth constraints, SyncCast selects, among these paths, the one which will lead to the smallest intra-session skew.

3. If there is no path satisfying either constraints, SyncCast heuristically selects the bandwidth-unconstrained path with the smallest intra-session skew.

We call the paths assigned in the above three cases unsuccessful paths.

During the stage of the multi-tree adjustment, SyncCast seeks alternative paths for the successful paths, to address of the issue of intra-session synchronization violation or bandwidth bottleneck for unsuccessful paths. It takes into account the following two situations.

1. If there is an intra-session synchronization violation, SyncCast finds alternative paths for the set of original successful paths that cause the unbounded synchronization skews.

2. If there are sites with unbounded incoming/outgoing bandwidth, SyncCast decides the alternative paths for the set of original successful paths that are routed through these bottlenecked sites.

After the multi-tree adjustment, if there are still unsuccessful paths violating the synchronization constraints, SyncCast will leave the problem to the presentation-tier synchronization controls. If some sites are still overloaded, the algorithm applies cooperative video frame rate allocations (Section 4.3.5).

\section{- Distribution of Video NDS in Class 2}

After the distribution of all video DS in class 1, SyncCast finds the paths for the set of video NDS to different receiver sites which have already been serviced as video DS to other receiver sites. Generally, for each video NDS, SyncCast first finds a set of parent site candidates such that the resulting path satisfies the intra-media synchronization constraint (across the video streams from the same sender to the same receiver). SyncCast then decides, among these candidates, the best parent site that has the lowest outgoing bandwidth usage that does not exceed the bandwidth upper bound.

\section{- Distribution of Video NDS in Class 3}

The distribution policy of video NDS in class 3 is almost the same as those in class 2 . The only exception is that a receiver site needs to request a video NDS directly from the original sender site, if the video stream has never been serviced. 


\subsubsection{Cooperative Frame Rate Allocation}

SyncCast applies the cooperative video frame rate allocation scheme to reduce and bound the incoming/outgoing bandwidth overhead at the overloaded sites shared by multiple video DS. We suppose there are $W$ video DS $d s_{1}, \ldots, d s_{W}$ (from multiple sender sites to different receivers) that are relayed by an overloaded site $n^{x}$ with a preset upper bound $\max B_{\text {in }}^{x}$ and $\max B_{\text {out }}^{x}$. We also assume the frame rate of each video $\mathrm{DS}$ is $\mathrm{FR}_{1}, \ldots, \mathrm{FR}_{W}$.

Hence, if the total outgoing bandwidth usage at $n^{x}$ exceeds the upper bound $\left(W>\max B_{\text {out }}^{x}\right.$ ), the actual frame rate for each video DS should be reduced to $\mathrm{FR}_{1} \times\left(\max B_{\text {out }}^{x}\right) / W, \ldots, \mathrm{FR}_{W} \times\left(\max B_{\text {out }}^{x}\right) / W$. Similarly, if the total incoming bandwidth usage at $n^{x}$ exceeds the upper bound ( $W>\max B_{\text {in }}^{x}$ ), the actual frame rate for each video DS will be reduced to $\mathrm{FR}_{1} \times\left(\max B_{\text {in }}^{x}\right) / W, \ldots, \mathrm{FR}_{W} \times\left(\max B_{\text {in }}^{x}\right) / W$.

\subsubsection{Evaluation Results}

\section{- Description of Evaluation Testbed}

We develop a multi-site 3DTI simulator for SyncCast evaluations, and we consider both 5-site and 9-site cases. For each case, we evaluate different connectivity setups with participants sites in different continents of the world (Table 4.1 and 4.2). These connectivity setups can be broadly divided into three scenarios based on their geographical locations: (1) all sites in US (represented as 5A and 9A), (2) sites in both US and Europe (5B and 9B), and (3) sites in US, Europe and Asia (5C and 9C). We obtain the average one-way latency statistics for each setup from real PlanetLab nodes. To get the realistic latency data, we send UDP packets from each site to all others at the same time and computed the one-way latency by halving the roundtrip time information. The size of the packets was smaller than the maximum transmission unit (MTU) in order to avoid fragmentation. In addition, widely-deployed firewall and poor link conditions (high loss rate, large jitter or low data rate) can impede the direct connections and transmissions of real-time traffic between certain sites. Hence, we remove some of the links (shown in the grey boxes in Table 4.1 and 4.2), and represent the new network environment as 5D-5F and 9D-9F to simulate the firewall-blocked connection (e.g. the link between IL and IN in Table 4.1(a)) or poor link conditions (e.g., links between Beijing and all sites outside China in Table 4.2(c)). So there are total of 12 connectivity setups for evaluation.

We suppose each sender site outputs 8 video streams from 8 3D cameras placed evenly (in a separation of 45-degree angle) in a 360-degree circle around a scene [12]. These cameras can capture the 3D images of the same scene from different views. From each sender site, a receiver requests 1 audio stream, 1 video 
Table 4.1: Average one-way delay (msec) for 5 nodes. (a) in US, (b) in US and Europe, (c) in US, Europe and Asia.

\begin{tabular}{|l|l|l|l|l|l|}
\hline \multicolumn{7}{|l|}{ Table (a): 5 nodes in US (5A,5D) } \\
\hline 5A & CA & IL & FL & IN & NY \\
\hline CA & 0 & 32 & 35 & 33 & 43 \\
\hline IL & 32 & 0 & 28 & 10 & 21 \\
\hline FL & 35 & 28 & 0 & 24 & 24 \\
\hline IN & 33 & 10 & 24 & 0 & 18 \\
\hline NY & 43 & 21 & 24 & 18 & 0 \\
\hline
\end{tabular}

\begin{tabular}{|l|l|l|l|l|l|}
\hline \multicolumn{6}{|c|}{ Table (b): 5 nodes in US and Europe (5B,5E) } \\
\hline 5B & CA & IL & UK & DE & NY \\
\hline CA & 0 & 32 & 80 & 88 & 43 \\
\hline IL & 32 & 0 & 72 & 75 & 21 \\
\hline UK & 80 & 72 & 0 & 18 & 53 \\
\hline DE & 88 & 75 & 18 & 0 & 52 \\
\hline NY & 43 & 21 & 53 & 52 & 0 \\
\hline
\end{tabular}

\begin{tabular}{|l|l|l|l|l|l|}
\hline \multicolumn{6}{|c|}{ Table (c): 5 nodes in US, Europe and Asia (5C,5F) } \\
\hline 5C & CA & IL & DE & JP & CN \\
\hline CA & 0 & 32 & 88 & 70 & 152 \\
\hline IL & 32 & 0 & 75 & 109 & 178 \\
\hline DE & 88 & 75 & 0 & 140 & 123 \\
\hline JP & 70 & 109 & 140 & 0 & 34 \\
\hline CN & 152 & 178 & 123 & 34 & 0 \\
\hline
\end{tabular}

DS and 2 video NDS (which are the two neighboring streams of the video DS). To simulate a real multi-site collaboration scenario, we suppose $50 \%$ of the receiver sites share the same video DS, and the other $50 \%$ of the receiver sites request different video DS. We omit other media modalities because they will not affect the SyncCast topology construction.

We set both incoming and outgoing bandwidth upper bound to be 10 video streams at each site (i.e., $\left.\max B_{\text {in }}^{x}=10, \max B_{\text {out }}^{x}=10\right)$. We prescribe that the intra-media skew upper bound $\delta_{1}(\mathrm{~V})=80 \mathrm{~ms}$ and that the intra-session skew upper bound $\delta_{3}$ varies from 100 to $300 \mathrm{~ms}$ (in a separation of $100 \mathrm{~ms}$ ).

To compare SyncCast with ViewCast, we use the same intra-session skew upper bound $\delta_{3}$ for both SyncCast and ViewCast. In ViewCast, we achieve this by setting ViewCast's $T_{\text {net }}$ upper bound equal to $\delta_{3}$.

\section{- Simulation Results}

Here, we only present the situation that $50 \%$ of the participating sites are sender sites (i.e., 2 sender sites in the 5-site case and 4 sender sites in the 9 -site case). The set of the senders are randomly selected in each 
Table 4.2: One-way delay (msec) for 9 nodes. (a) in US, (b) in US and Europe, (c) in US, Europe and China.

\begin{tabular}{|c|c|c|c|c|c|c|c|c|c|}
\hline \multicolumn{1}{|c|}{ Table (a): 9 nodes in US (9A,9D) } \\
\hline 9A & CA1 & CA2 & IL1 & IL2 & IL3 & FL & IN & NY & TX \\
\hline CA1 & 0 & 2 & 32 & 31 & 32 & 35 & 33 & 43 & 18 \\
\hline CA2 & 2 & 0 & 31 & 35 & 32 & 35 & 20 & 46 & 19 \\
\hline IL1 & 32 & 31 & 0 & 1 & 2 & 28 & 10 & 21 & 19 \\
\hline IL2 & 31 & 35 & 1 & 0 & 1 & 28 & 11 & 21 & 19 \\
\hline IL3 & 30 & 32 & 2 & 1 & 0 & 28 & 11 & 21 & 20 \\
\hline FL & 35 & 35 & 28 & 28 & 28 & 0 & 24 & 24 & 27 \\
\hline IN & 33 & 20 & 10 & 11 & 11 & 24 & 0 & 18 & 21 \\
\hline NY & 43 & 46 & 21 & 21 & 21 & 24 & 18 & 0 & 31 \\
\hline TX & 18 & 19 & 19 & 19 & 20 & 27 & 21 & 31 & 0 \\
\hline
\end{tabular}

\begin{tabular}{|c|c|c|c|c|c|c|c|c|c|}
\hline \multicolumn{2}{|c|}{ Table (b): 9 nodes in US and Europe (9B,9E) } \\
\hline 9B & CA & IL1 & IL2 & FL & IN & UK & DE1 & DE2 & IT \\
\hline CA & 0 & 32 & 31 & 35 & 33 & 80 & 88 & 90 & 102 \\
\hline IL1 & 32 & 0 & 1 & 28 & 10 & 72 & 75 & 81 & 95 \\
\hline IL2 & 31 & 1 & 0 & 28 & 11 & 74 & 72 & 85 & 98 \\
\hline FL & 35 & 28 & 28 & 0 & 24 & 85 & 86 & 82 & 92 \\
\hline IN & 33 & 10 & 11 & 24 & 0 & 70 & 71 & 76 & 93 \\
\hline UK & 80 & 72 & 74 & 85 & 70 & 0 & 18 & 18 & 24 \\
\hline DE1 & 88 & 75 & 72 & 86 & 71 & 18 & 0 & 6 & 17 \\
\hline DE2 & 90 & 81 & 85 & 82 & 76 & 18 & 6 & 0 & 21 \\
\hline IT & 102 & 95 & 98 & 92 & 93 & 24 & 17 & 21 & 0 \\
\hline
\end{tabular}

\begin{tabular}{|c|c|c|c|c|c|c|c|c|c|}
\hline \multicolumn{10}{|c|}{ Table (c): 9 nodes in US, Europe and China (9C,9F) } \\
\hline 9C & CA & IL1 & IL2 & UK & DE1 & DE2 & SH & BJ & HK \\
\hline CA & 0 & 32 & 31 & 80 & 88 & 90 & 152 & 193 & 167 \\
\hline IL1 & 32 & 0 & 1 & 72 & 75 & 81 & 178 & 204 & 179 \\
\hline IL2 & 31 & 1 & 0 & 74 & 72 & 85 & 175 & 203 & 182 \\
\hline UK & 80 & 72 & 74 & 0 & 18 & 18 & 155 & 187 & 160 \\
\hline DE & 88 & 75 & 72 & 18 & 0 & 6 & 143 & 181 & 152 \\
\hline DE & 90 & 81 & 85 & 18 & 6 & 0 & 140 & 180 & 153 \\
\hline SH & 152 & 178 & 175 & 155 & 143 & 140 & 0 & 61 & 55 \\
\hline BJ & 193 & 204 & 203 & 187 & 181 & 180 & 61 & 0 & 87 \\
\hline HK & 167 & 179 & 182 & 160 & 152 & 153 & 55 & 87 & 0 \\
\hline
\end{tabular}




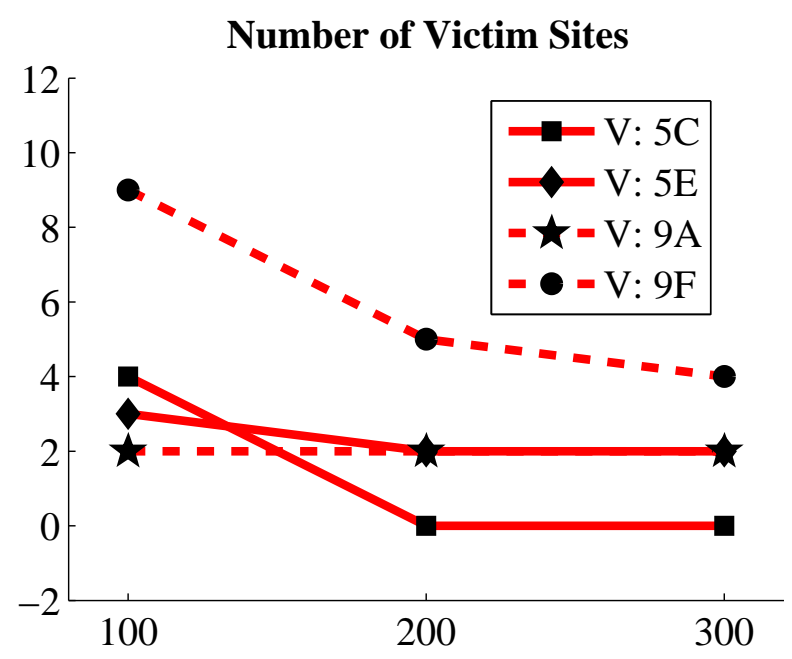

Figure 4.8: Number of victim sites in ViewCast. X-axis represents $\delta_{3}$. Number of victim sites in SyncCast is always zero.

connectivity setup.

Victims. Fig. 4.8 shows the number of victim sites in ViewCast. The victims are incurred due to the fact that (1) ViewCast does not take into account the service class differentiation, so video NDS can consume lots of bandwidth resources which would otherwise used for video DS; and that (2) the $T_{\text {net }}$ upper bound can reduce the video DS path availability, so the number of victim sites increases as the $T_{\text {net }}$ upper bound decreases. By contrast, there are no victim sites introduced by SyncCast because of its mechanisms of the service class differentiation, the cooperative bandwidth allocation and the path selection policies. Fig. 4.9(a)-(b) demonstrate that the number of victim streams are also consistently lower than ViewCast.

Bandwidth overhead. It is not difficult to prove that the average incoming and outgoing bandwidth overhead per site should be equal, so we present the results using the same figure. Fig. 4.9(c)-(d) demonstrate that SyncCast boasts a higher incoming/outgoing bandwidth utilization than ViewCast, which is the main cause for less victim streams/sites in SyncCast.

Average $T_{\text {net }}$ of video DS paths. Fig. 4.9(e)-(f) depict the average $T_{n e t}$ of video DS paths of the two algorithms. When $\delta_{3}=200$ or $300 \mathrm{~ms}$, SyncCast's results are comparable to or smaller than ViewCast's, proving the effectiveness of the SyncCast algorithm. When $\delta_{3}=100 \mathrm{~ms}$, due to huge number of victim sites in ViewCast, the remaining successful video DS paths (usually with shorter $T_{n e t}$ ) can lead to an average smaller than SyncCast's results.

Intra-media skews for videos. We compare the intra-media skews for videos in Fig. 4.10(a)-(b). We show that SyncCast can consistently achieve the intra-media synchronization within the preset $80 \mathrm{~ms}$ con- 


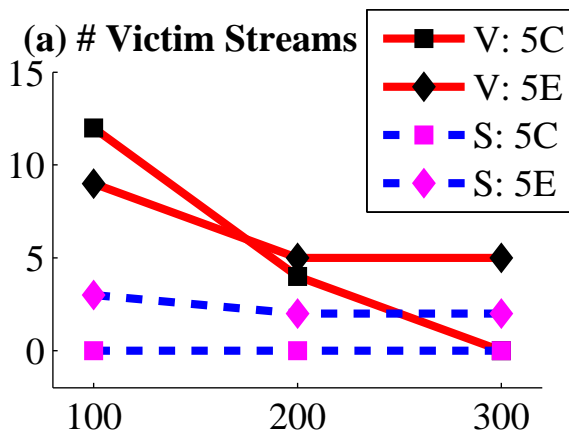

(c) Mean BW
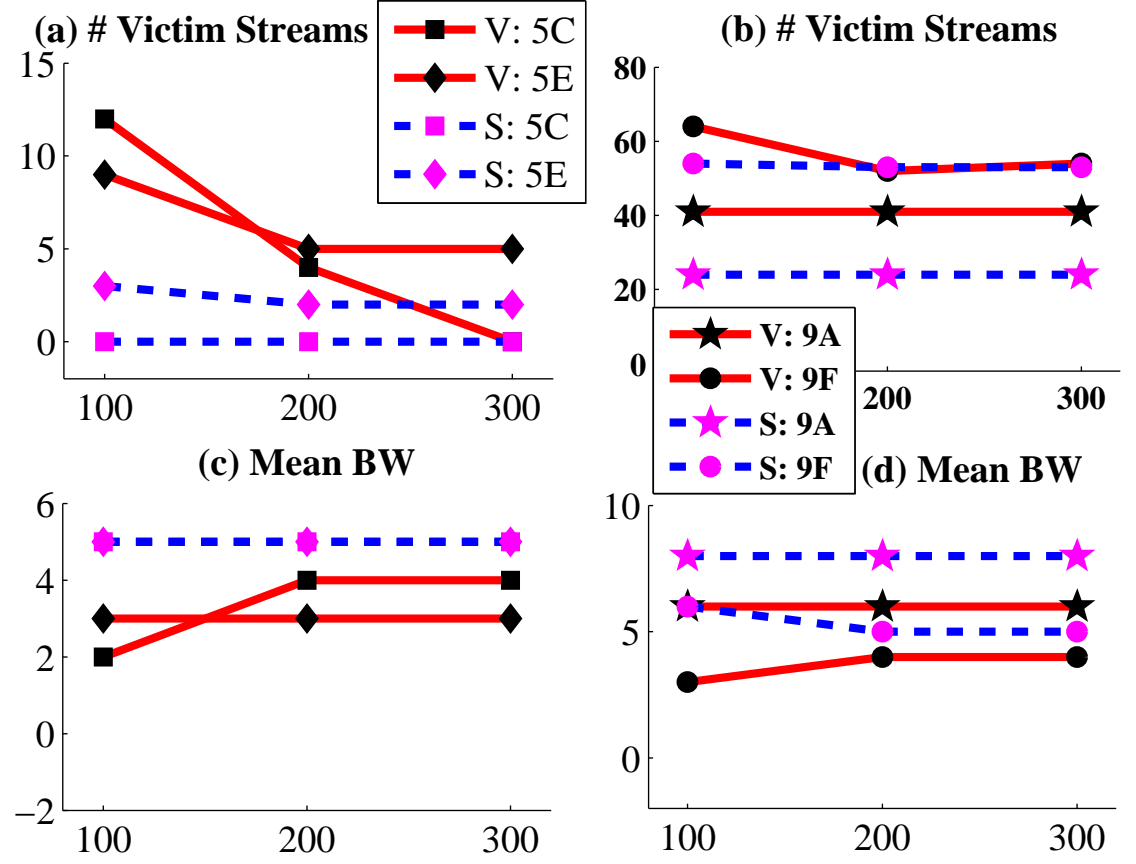

(e) Mean $T_{\text {net }}$
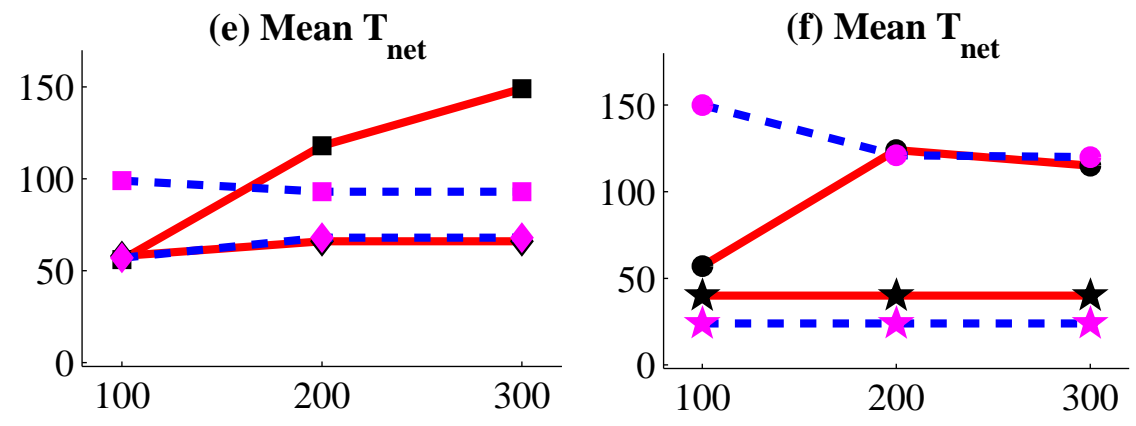

Figure 4.9: SyncCast/ViewCast. X-axis represents $\delta_{3}$. 
(a) Max Intra-media Skew

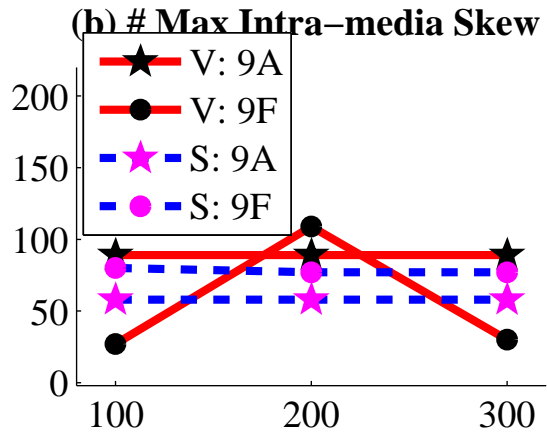

100

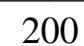

300

(d) Max Inter-sender Skew
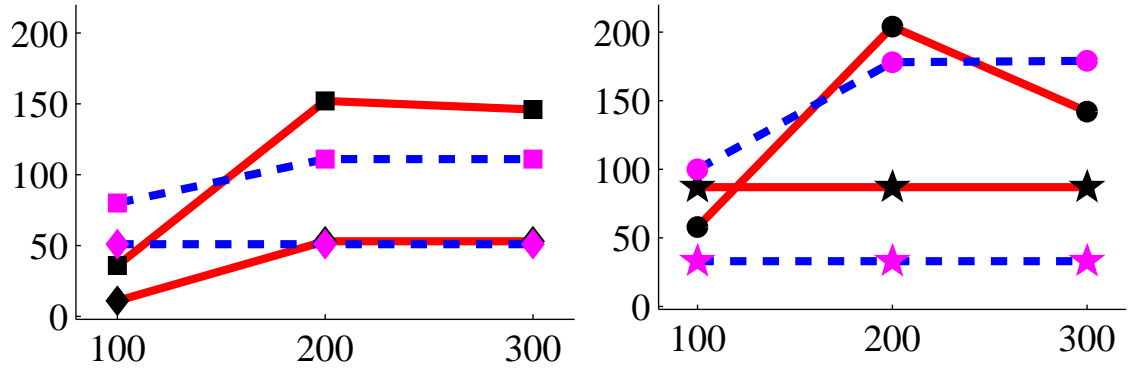

(e) Max Inter-receiver Skew

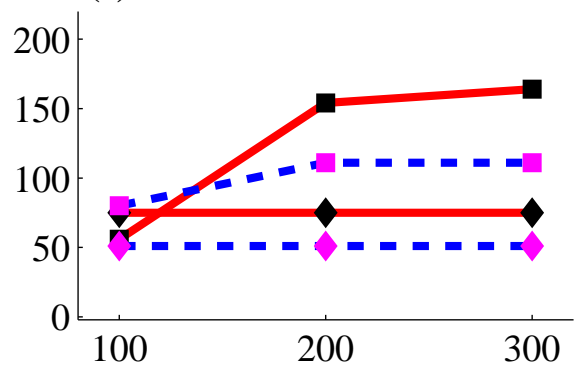

(f) Max Inter-receiver Skew

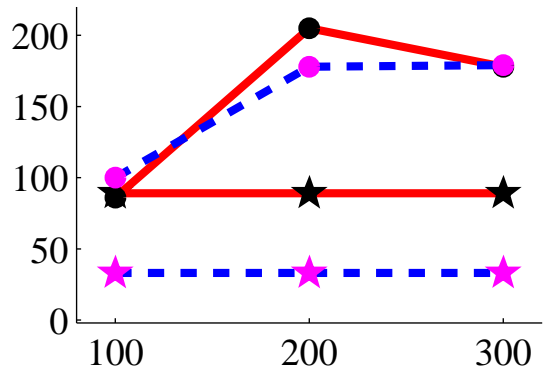

Figure 4.10: SyncCast/ViewCast. X-axis represents $\delta_{3}$. 


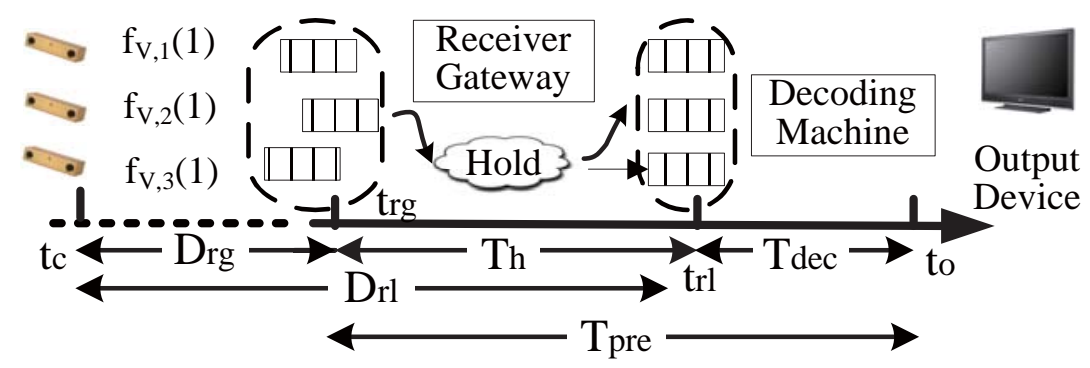

Figure 4.11: Timeline at the receiver gateway

straint by dropping video streams which would otherwise cause unbounded skews. On the other hand, ViewCast does not demand this constraint, so the resulting skews can only be limited within the $T_{n e t}$ upper bound.

Intra-session skews. Fig. 4.10(c)-(f) show the maximum intra-session skews (both inter-sender and inter-receive). They depict that both ViewCast and SyncCast can successfully limit all the intra-session skews within the prescribed upper bound. Note that because the two protocols are using different approaches to achieve the synchronization, the resulting intra-session skews of SyncCast do not have to be smaller than those of ViewCast.

\subsection{TBuffer: Presentation Tier Control}

We develop TBuffer, an adaptive media presentation scheduling (MPS) scheme for multi-device synchronization at each teleimmersive receiver. We focus on the inter-sender skews at the intra-session layer in our study, and assume that the inter-receiver synchronization problem has been solved by existing studies $[82,83,84,85]$.

Each receiver site consistently monitors the multi-layer synchronization skews based on the intra-bundle synchronization point (SP) sequences (Section 3.3) from multiple senders. To achieve in-sync media presentation at the distributed output devices at a receiver, existing studies $[15,19]$ usually employ MPS to compensate for the media delay difference within/across the bundles by performing buffering adaptation. However, distributed buffer control at scalable media output devices requires expensive message communication. In our study, we perform multimedia synchronization by referencing the skews measured at the intra-bundle SP sequences at each receiver gateway. 


\subsubsection{Problem Formulation}

We denote the (global) arrival time of media data at a receiver gateway as $t_{r g}$, the (global) release time by the gateway at $t_{r l}$ and their (global) presentation time at output devices $t_{o}$ (Fig. 4.11). Hence, $D_{r g}=$ $t_{r g}-t_{c}=T_{c a p}+T_{n e t}$ is the media latency incurred at the sender and Internet transmission, $T_{h}=t_{r l}-t_{r g}$ is the media hold (buffering) time at the gateway, $T_{d e c}=t_{o}-t_{r l}$ is the computation (decoding) and processing time incurred at the output devices, and $T_{p r e}=T_{h}+T_{d e c}$ is the overall time incurred at the presentation tier. We fix a constant $T_{d e c}$ for all media modalities in our system to reduce message exchanges. Our goal is to decide $t_{r l}$ (and $T_{h}$ ) based on $D_{r g}$ for multi-layer media synchronization. In other words, the synchronization skews of $D_{r l}=t_{r l}-t_{c}$ must be bounded across multi-modal multi-stream data of all media bundles at each receiver.

Due to the real-time nature of teleimmersion, a longer $D_{r l}$ means a larger hold time or buffering latency: accommodating greater variations of $D_{r g}$ at the expense of poorer interactivity. Here, we aim to decide the minimal $D_{r l}$ for each multi-modal sensory stream within the session, which can successfully smooth Internet jitter and bound the resulting multi-layer synchronization skews (evaluated at the sequence of intrabundle synchronization point from each sender site). For a given receiver $r^{00}$, the following criteria must be satisfied:

$$
\begin{aligned}
\forall x, i, j: \min D_{r l}\left(s_{i, j}^{x}, n^{y_{0}}\right) \text { s.t. } & \\
P\left(D_{r l}\left(s_{i, j}^{x}, n^{y_{0}}\right) \leq D_{r g}\left(f_{i, j}^{x}(k), n^{y_{0}}\right)\right) & \leq \mathrm{UR}_{\mathrm{TH}}(i) \\
\left|\Delta D_{r l}\left(s_{i, j}^{x}, n^{y_{0}}\right)\right| & \leq \delta_{1}(i) \\
\left|\Delta D_{r l}\left(m_{i}^{x}, n^{y_{0}}\right)\right| & \leq \delta_{2}(i, *) \\
\left|\Delta D_{r l}\left(u^{x}, n^{y_{0}}\right)\right| & \leq \delta_{3}
\end{aligned}
$$

In the above equations, Eqn. 4.5 specifies all media frames within a sensory stream $s_{i, j}^{x}$ should maintain a same $D_{r l}$ to guarantee intra-stream synchronization. We count within each stream the number of media frames that should have arrived at $n^{y_{0}}$, and Eqn. 4.6 indicates the maximal unavailable rate/percentage (UR) of these media frames at their scheduled release time $t_{r l}$, where $\mathrm{UR}_{\mathrm{TH}}(i)$ is the upper bound of the unavailability of the media modality $i$. Eqn. 4.7-4.9 specify the multi-layer skews, and the prescribed skew upper bound for each layer: $\delta_{1}(i), \delta_{2}(i, *)$ and $\delta_{3}$ (defined in Section 4.3.2). 


\subsubsection{Design of TBuffer}

At each receiver site $n^{y_{0}}$, we decide $D_{r l}$ (and the resulting $T_{h}$ ) of all received sensory streams using the following three steps.

Step 1. We first decide the lower bound of $D_{r l}\left(s_{i, j}^{x}, n^{y_{0}}\right)$ for each sensory stream $s_{i, j}^{x}$ to satisfy Eqn. 4.6, and denote it as $\hat{D}_{r l}\left(s_{i, j}^{x}, n^{y_{0}}\right)$. This can be achieved by recording the arrival time of media frames at each intra-bundle synchronization point (from different senders) since the last system update. We sort the list of $\left\{\hat{D}_{r l}\left(s_{i, j}^{x}, n^{y_{0}}\right)\right\}_{x, i, j}$ within the whole session from the longest to the shortest, and denote $\left\{\tilde{s}_{q}\right\}$ as the resulting ordered sensory streams corresponding to the sorted $\left\{\hat{D}_{r l}\right\}$ (i.e., $\forall q<q^{\prime}: \hat{D}_{r l}\left(\tilde{s}_{q}, n^{y_{0}}\right)>$ $\left.\hat{D}_{r l}\left(\tilde{s}_{q^{\prime}}, n^{y_{0}}\right)\right)$.

Step 2. Based on $\left\{\hat{D}_{r l}\right\}$, we then update the actual $D_{r l}\left(D_{r l} \geq \hat{D}_{r l}\right)$ for each sensory stream to satisfy the multi-layer skew bound within/across multiple bundles from all sender sites. For each stream $\tilde{s}_{q}$, we evaluate the multi-layer synchronization skews introduced by $\tilde{s}_{q}$ and $\tilde{s}_{q^{\prime}}\left(\forall q^{\prime}=\{1, \ldots, q-1\}\right)$. If the synchronization satisfies Eqn. 4.7-4.9 constraints, we let $D_{r l}\left(\tilde{s}_{q}, n^{y_{0}}\right)=\hat{D}_{r l}\left(\tilde{s}_{q}, n^{y_{0}}\right)$. Otherwise, we increase the hold time of $\tilde{s}_{q}$ and set $D_{r l}\left(\tilde{s}_{q}\right)$ to be the smallest number which does not violate the synchronization demand. In each iteration, we go through all sensory streams within the same session, and update the list $\left\{D_{r l}\right\}$. Note that because of the multi-layer synchronization dependencies, the algorithm may have to carry on multiple iterations before the multi-layer synchronization demands specified in Eqn. 4.7-4.9 are completely satisfied.

Step 3. Finally, we set the hold time of all media frames within each sensory stream, so that they are shared with the same $D_{r l}$. Based on their captured time $t_{c}$, we can compute $t_{r l}$ and $T_{h}$ accordingly. The above approach will return a minimal solution list $\left\{D_{r l}\right\}$ that satisfies all criteria as specified in Eqn. 4.5-4.9.

\subsubsection{Evaluation Results}

\section{- Descriptions of Evaluation Testbed}

TEEVE testbed. We rely on the real teleimmersive testbed TEEVE, to evaluate the our TBuffer scheme. One audio stream and up to three 3D video streams (representing three views) are configured at each site. To make our results repeatable, we prerecord at the senders the size of original audio and multiview video frames, so that our testbed is able to transmit the same media data in each experiment. ${ }^{2}$ A network emulator is implemented between the sender and receiver gateways. It can replay delay and loss distributions for the

\footnotetext{
${ }^{2}$ We will evaluate real-time video and audio traffics and the resulting presentation scheduling in teleimmersion in Section 6.
} 
teleimmerive audio and video packets according to the real multimedia traces collected in the Internet. To evaluate the bandwidth impact, we use $t c$ software to control the upper bound of the data rate at the sender gateways.

PlanetLab traces. We collect the Internet traces from the PlanetLab environment, as the input of our network emulator. To make the results trustworthy, the PlanetLab sender uses UDP to send real teleimmersive audio and video packets piggybacking local timestamp. The data rate of the audio stream is $100 \mathrm{kbps}$, and of each video stream is around 3-4 Mbps. The PlanetLab receiver then collects the received media traces, and record their one-way delay and loss distributions which will be used in the TEEVE testbed. Up to 3 video streams are evaluated. To mitigate the bandwidth impact on the Internet delays and losses, we carefully select PlanetLab computers which do not have bandwidth constraints. We then classify the Internet connections based on the network statistics, so that our TEEVE testbed can be evaluated in diverse Internet environment.

\section{- Analysis of Experiment Results}

To show the effectiveness of our TBuffer algorithm, we present a simple case with two sites, where the sender site 1 sends three video streams and one audio stream to the receiver site 2. The PlanetLab traffic is collected between USA and China in 2011. We compare our algorithm (Alg 1) with the traditional single-reference inter-stream synchronization studies (Alg 2) [19]. In our Alg 1, we choose $\xi_{V, 3}^{1}$ and $s_{A, 1}^{1}$ as the video/audio reference stream, and audio $m_{A}^{1}$ as the reference modality. In previous Alg 2, we simply choose $s_{A, 1}^{1}$ as the synchronization reference for all video streams. Fig. 4.12 and 4.13 show the results over a 40 -sec span, for $\mathrm{UR}_{\mathrm{TH}}\left({ }^{\prime} \mathrm{V}\right.$ ') $=4 \%, \mathrm{UR}_{\mathrm{TH}}\left({ }^{\prime} \mathrm{A}\right.$ ') $=1.5 \%, \delta_{1}\left({ }^{\prime} \mathrm{V}^{\prime}\right)=\delta_{1}\left({ }^{\prime} \mathrm{A}\right.$ ') $=0$, and $\delta_{2}\left({ }^{\prime} \mathrm{V}^{\prime}, *\right)=$ $\delta_{2}\left({ }^{\prime} \mathrm{V}\right.$ ', 'A') $=80 \mathrm{~ms}$ in Eqn. 17-20 (we select these data based on [1]). We demand zero intra-media skew (i.e., $\forall i, \delta_{1}(i)=0$ ) for minimal spatial distortion of every media modality. We update the control status every $W=8$ seconds.

Fig. 4.12 shows the $D_{r g}$ and the resulting $D_{r l}$ adaptations for the four sensory streams. Between two consecutive updates, the intra-stream synchronization is guaranteed by maintaining equal $D_{r l}$ of all media frames within each sensory stream. Fig. 4.13(a)(b) present the intra-media and intra-bundle synchronization skews $\Delta D_{r g}$ due to Internet dynamics. Fig. 4.13 (c)(d) demonstrate the resulting multi-layer skews of $\Delta D_{r l}$ after the MPS control at the receiver gateway. There is no doubt that the traditional single-reference algorithm (Alg 2) is unable to effectively bound the skews across the video streams, while our SP-based algorithm can successfully and consistently achieve it by relying on the hierarchical synchronization references. We have found that only less than $2 \%$ of intra-bundle SP do not arrive in time using our algorithm, 


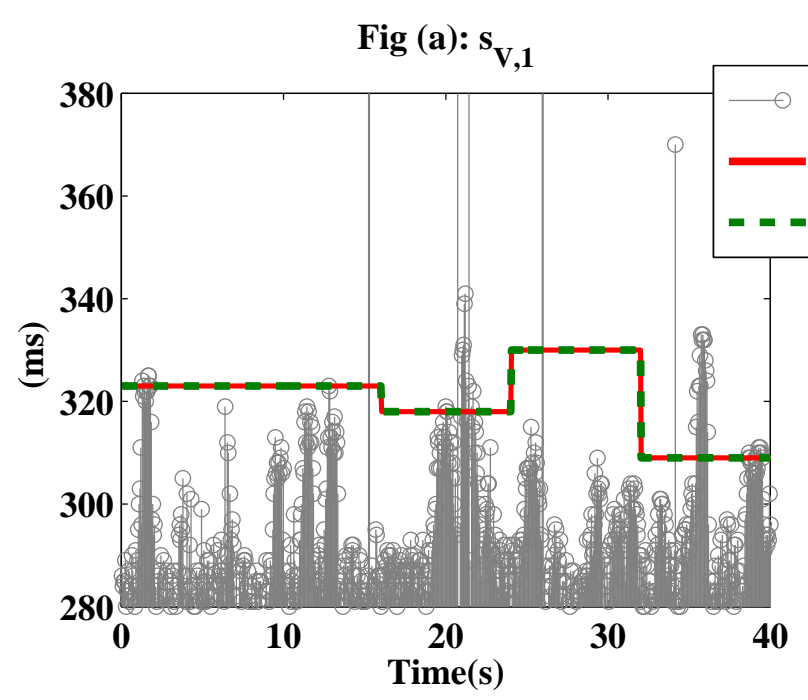

Fig (c): $s_{V, 3}$

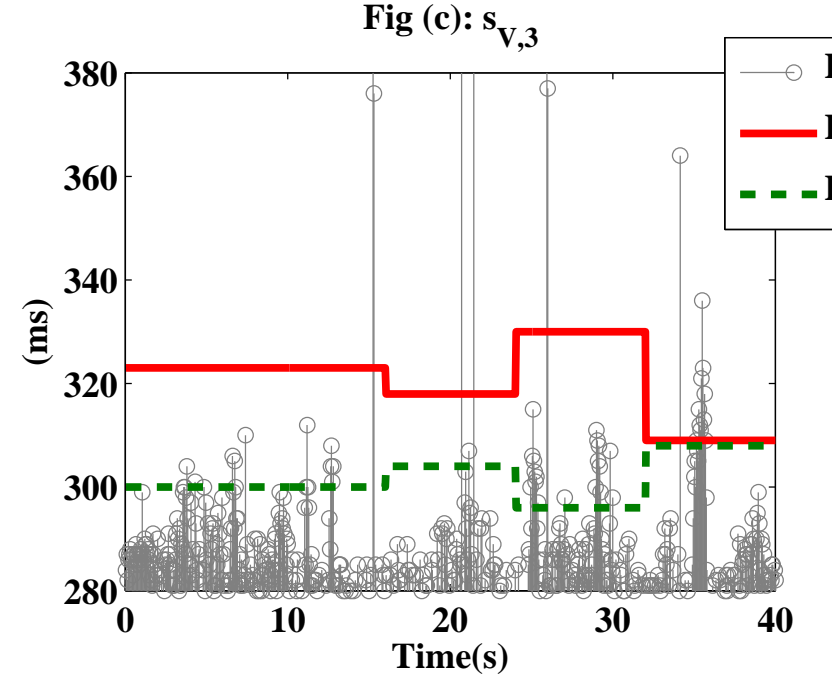

Fig (b): $\mathrm{s}_{\mathrm{v}, 2}$

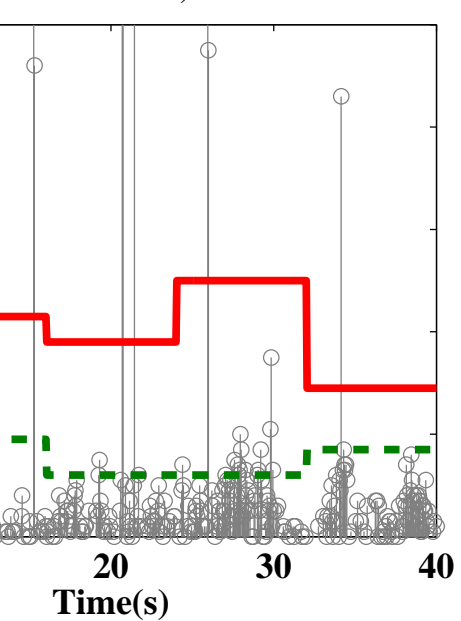

Fig (d): $s_{A, 1}$

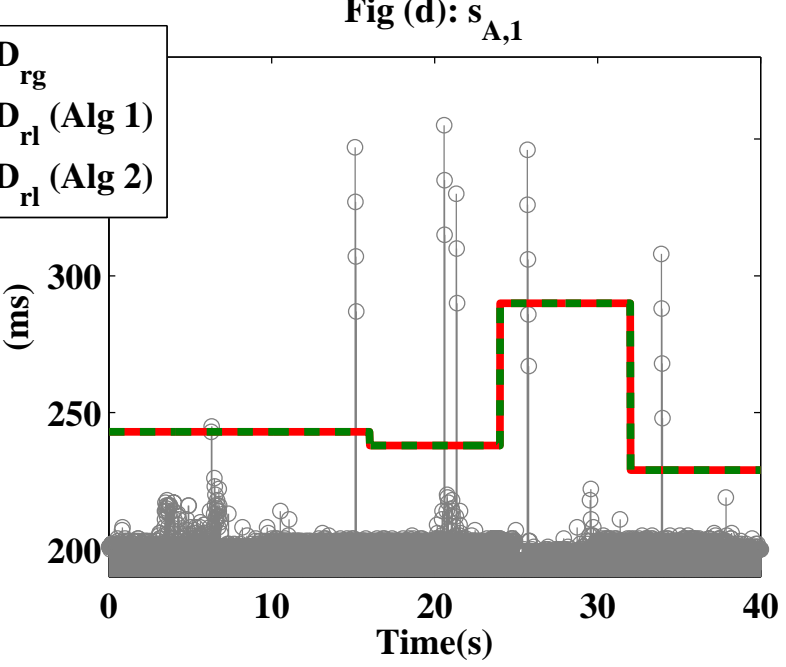

Figure 4.12: $D_{r g}$ of each sensory stream evaluated at intra-bundle synchronization point, and the resulting $D_{r l}$ adaptation. Alg 1: our TBuffer algorithm; Alg 2: traditional single-reference algorithm. Note that in (a),(d), the two $D_{r l}$ curves of Alg 1 and 2 overlap. Superscript indicating the sender site is omitted in all denotations in the figure. 
Fig (a)

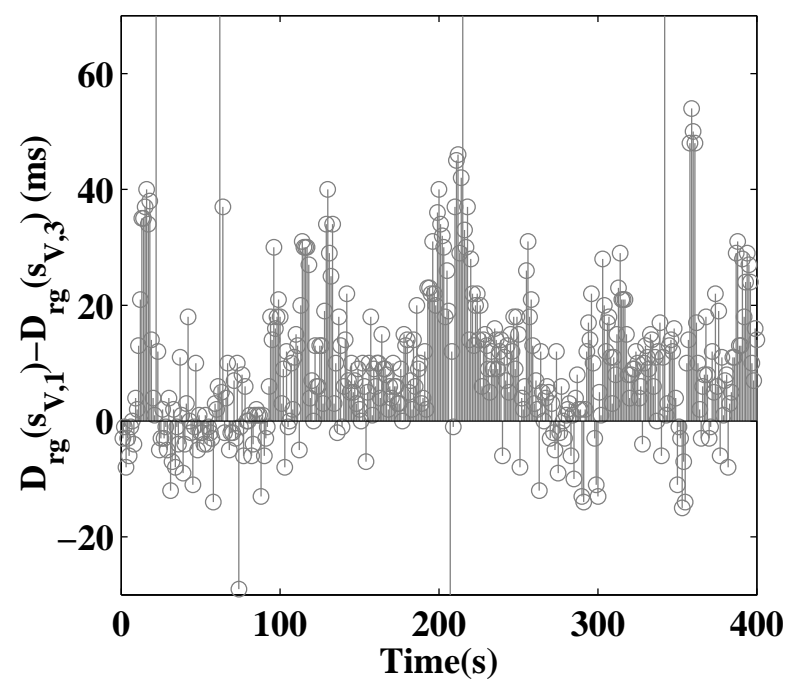

Fig (b)

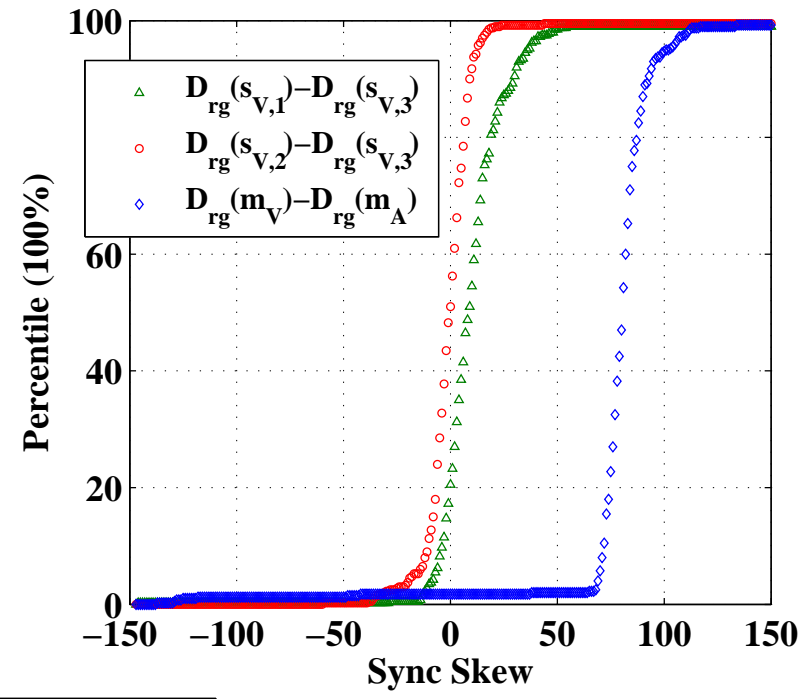

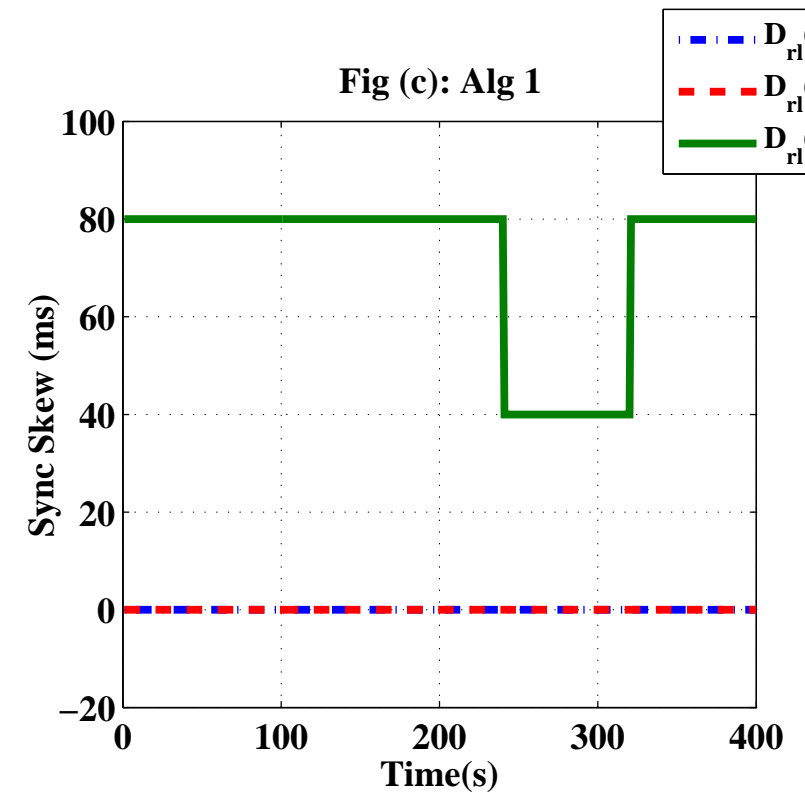

Fig (d): Alg 2

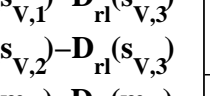
:

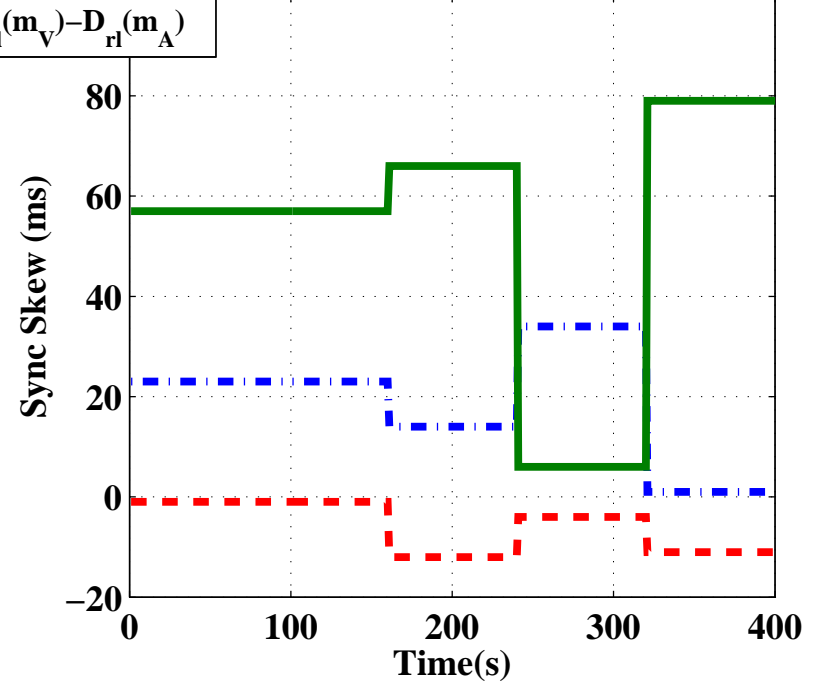

Figure 4.13: $s_{V, 3}^{1}$ is the video reference stream. (a) Snapshot of $\Delta D_{r g}\left(s_{V, 1}^{1}\right)$; (b) Percentile of synchronization skew: $\Delta D_{r g}\left(s_{V, 1}^{1}\right), \Delta D_{r g}\left(s_{V, 2}^{1}\right)$ and $\Delta D_{r g}\left(m_{V}^{1}\right)$; (c-d) Resulting synchronization skew due to receiver adaptation: $\Delta D_{r l}\left(s_{V, 1}^{1}\right), \Delta D_{r l}\left(s_{V, 2}^{1}\right)$ and $\Delta D_{r l}\left(m_{V}^{1}\right)$. Alg 1: our TBuffer algorithm; Alg 2: traditional single-reference algorithm. In (c), $\Delta D_{r l}\left(s_{V, 1}^{1}\right)=\Delta D_{r l}\left(s_{V, 2}^{1}\right)=0$. Superscript indicating the sender site is omitted in all denotations in the figure. 
and the number depends on the unavailability threshold, $\mathrm{UR}_{\mathrm{TH}}(i)$, that we have chosen for the evaluation.

\subsection{Summary}

In this chapter, we present a multi-tier collaboration synchronization control framework that is designed to constrain heterogeneous synchronization demands in each tier. The framework features (1) CloudStream, a cloud-based encoding parallelization and scheduling scheme for bounding the computation overhead and constraining the resulting computation skews at the capture tier; (2) SyncCast, a synchronized multi-source multicast overlay design at the distribution tier; and (3) TBuffer, a multi-modal multi-stream presentation scheduling scheme for multi-device synchronization at the presentation tier. All these control studies are developed without the human perceptual feedback. In the next section, we will focus on the perceptiondriven synchronization control approach.

\subsection{Table of Abbreviations and Notations}

\section{List of Abbreviations in Chapter 4:}

TEEVE Teleimmersive Environment for EVErybody

SP Synchronization Point

TCP Transmission Control Protocol

UDP $\quad$ User Datagram Protocol

IP Internet Protocol

EED End-to-End Delay

MPS Media Presentation Scheduling

\section{List of Notations in Chapter 4:}

$\begin{array}{ll}x & \text { Index of sender site } \\ i & \text { Index of media modality } \\ j & \text { Index of sensory stream } \\ k & \text { Index of media frame }\end{array}$




\begin{tabular}{|c|c|}
\hline$n^{x}$ & Sender site $x$ \\
\hline$N$ & Number of all sites in the session \\
\hline$N_{A P}$ & Number of activity participants (senders) in the session \\
\hline$t_{c}$ & Assigned captured time at the media sensor \\
\hline$t_{s g}$ & Arrival time at the sender gateway \\
\hline$t_{r g}$ & Arrival time at the receiver gateway \\
\hline$t_{r l}$ & Release time by the receiver gateway hold control \\
\hline$t_{o}$ & Media presentation time at the output devices \\
\hline$T_{c a p}$ & $T_{c a p}=t_{s g}-t_{c}$ Duration at the capturing tier \\
\hline$T_{n e t}$ & $T_{n e t}=t_{r g}-t_{s g}$ Duration at the distribution tier \\
\hline$T_{\text {pre }}$ & $T_{p r e}=t_{o}-t_{r g}$ Duration at the presentation tier \\
\hline$\Delta T_{\text {cap }}$ & Synchronization skew incurred at the capturing tier \\
\hline$\Delta T_{n e t}$ & Synchronization skew incurred at the distribution tier \\
\hline$\Delta T_{\text {pre }}$ & Synchronization skew incurred at the presentation tier \\
\hline$L$ & Linear predictor order \\
\hline$a_{l}$ & Linear predictor coefficient \\
\hline$\tilde{T}_{c a p}$ & Predicted computation time at the capturing tier \\
\hline$\Delta T_{\text {seq }}$ & Timing overhead at the sequential encoding part \\
\hline GCF & Global contribution factor \\
\hline$\vec{O}\left(s^{x} i, j\right)$ & Orientation of stream $s^{x} i, j$ \\
\hline$\vec{O}(x, y)$ & Desired receiver $n^{y}$ 's view orientation from $n^{x}$ 's streams \\
\hline$\zeta_{1}$ & Number of victim sites \\
\hline$\zeta_{2}$ & Number of victim streams \\
\hline$B_{\text {in }}^{x}$ & Ingoing bandwidth overhead at site $n^{x}$ \\
\hline$B_{\text {out }}^{x}$ & Outgoing bandwidth overhead at site $n^{x}$ \\
\hline $\max B_{\text {in }}^{x}$ & Maximum ingoing bandwidth availability at site $n^{x}$ \\
\hline $\max B_{\text {out }}^{x}$ & Maximum outgoing bandwidth availability at site $n^{x}$ \\
\hline$\delta_{1}(i)$ & Upper bound for intra-media synchronization skew of media modality $i$ \\
\hline$\delta_{2}(i, j)$ & Upper bound for intra-bundle synchronization skew between media modality $i$ and $j$ \\
\hline
\end{tabular}


$\delta_{3} \quad$ Upper bound for intra-bundle synchronization skew within the session

ds Dominant stream

W Number of video dominant streams

FR Frame rate

$T_{h} \quad T_{h}=t_{r l}-t_{r g}$ Hold duration at the receiver gateway

$T_{\text {dec }} \quad T_{d e c}=t_{o}-t_{r l}$ Decoding and processing expense at the decoding machine

$D_{r g} \quad D_{r g}=t_{r g}-t_{c}$ Latency of media data experienced before receiver gateway arrival

$D_{r l} \quad D_{r l}=t_{r l}-t_{c}$ Latency of media data experienced before receiver gateway release

$\Delta D_{r l} \quad$ Multi-layer synchronization skew at due to $D_{r l}$

$\mathrm{UR}_{\mathrm{TH}}(i) \quad$ Upper bound for unavailable rate for each sensory stream of media modality $i$

$\hat{D}_{r l} \quad$ Lower bound for $D_{r l}$ decided by $\operatorname{UR}_{\mathrm{TH}}(i)$ at receiver media presentation 


\section{CHAPTER 5}

\section{SYNCHRONIZATION AND HUMAN PERCEPTION}

In multimedia applications, the adaptation of synchronization control algorithms, and particularly the media presentation scheduling (MPS) at the receiver sites, decides a set of system operating statuses that will result in heterogeneous subjective user satisfactions. In turn, the human perception can serve as a direct feedback to guide the synchronization adaptation. Hence, in this chapter, we will conduct user study to evaluate the human perceptual quality as the result of the synchronization adaptation. We will also employ these real subjective feedback in designing the perception-driven synchronization approach.

\subsection{Overview}

Fig. 5.1 shows the overview of this chapter. We first study the modeling of teleimmersive applications, because the human perceptual quality is directly affected by the diverse functionalities of different shared activities during the teleimmersive collaborations. Based on the characteristics of each application, we investigate the objective quality metrics that capture both the streaming media quality and the flicker impact. These multidimensional objective metrics are able to be accessed in real time, so they can reasonably reflect the performance of the online synchronization adaptations. In addition, their combined contributions also decide the overall human perceptual quality. Hence, in order to understand the people's real feelings, we conduct the subjective user study and evaluate media samples with different multidimensional objective quality values, that are likely to appear during the synchronization adaptations in the real teleimmersive applications. We employ the comparative category rating (CCR) method (discussed in Section 2.2) in our study, with a goal to understand the quality comparison outcomes of media samples with different objective values. We show the CCR inconclusiveness under the multidimensional quality tradeoffs, and propose a new subjective metric to address the CCR issue. By generalizing the subjective evaluation results, we perform the perception-driven synchronization with MPS control, and decide system operating status with the best user satisfaction.

In our study, we simplify the problem by only investigating the two-party teleimmersive applications 


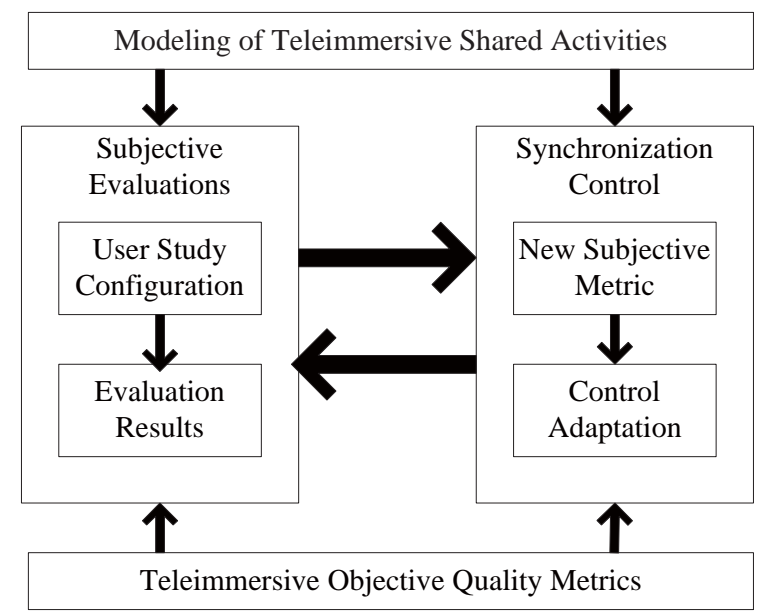

Figure 5.1: Overview of Chapter 5.

with both parties as active participants. We assume that each site outputs multiple multiview video streams and one single audio stream. We prescribe that the set of time-correlated video frames (intra-media synchronization points) from each sender should be played at the receiver rendering display at the same time, meaning that the intra-media synchronization is guaranteed.

\subsection{Studied Teleimmersive Shared Activities}

Depending on the shared interactive activities performed in different teleimmersive applications, a multimedia system can have diverse demands on various quality dimensions. Thus, it is interesting to understand how the perceptual quality is impacted by the heterogeneity of shared activities. In our study, we investigate two representative activities (applications): the conversation-oriented (CONV) tasks and the collaborative (COLL) gaming activities.

The CONV activity (application) describes the conferencing scenario with a social conversation, where participants at both sites are talking to each other with slow motion movement (Fig. 5.2(a)). Generally, users in CONV pay attention to the audio intelligibility of the conversation more than the image quality.

In COLL (Fig. 5.2(b)), two distributed participants are playing the "rock-paper-scissor" game in a virtual space. In this application, the visual timing mismatch is more important to the human perception.

\subsection{Objective Quality Metrics Impacting Human Perception}

By extracting the unique features of the two teleimmersive activities CONV and COLL, we identify the objective quality metrics that are able to characterize each dimension of the overall streaming media quality 


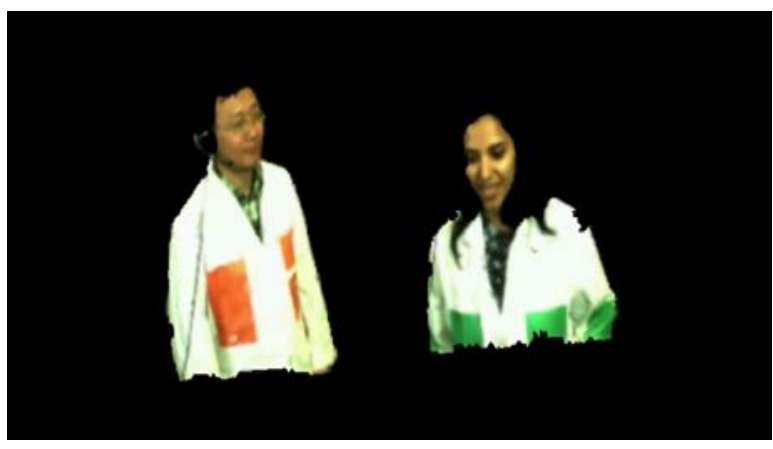

(a) CONV: conferencing scenario with social talk

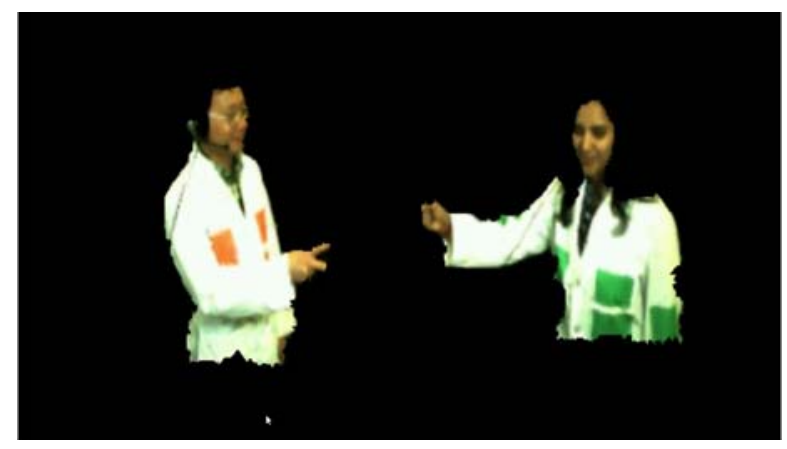

(b) COLL: rock-paper-scissor collaborative gaming

Figure 5.2: Two Teleimmersive applications evaluated in our user study.

and the resulting flicker effect.

\subsubsection{Objective Metrics for Streaming Media Quality}

\section{- Media Signal Intelligibility}

The media signal intelligibility in the teleimmersion includes the audio quality $e_{A}$ and the multiview video quality $e_{V}$. The two metrics can be degraded by jitter and losses over the wireline and wireless networks.

For both wideband and narrowband audios, we use the metric of Perceptual Evaluation of Speech Quality (PESQ) defined in ITU-T P.862 [157] to approximate $e_{A}$. PESQ allows the automatic computation of the quality of a (degraded) audio signal during the presence of the original reference. It returns a score ranging from 1 to 4.5. A larger PESQ means the (degraded) audio signal is more approximate to the reference, and hence a better audio intelligibility.

There are lots of factors deciding the multiview video intelligibility (rendered on the 2D screen): the multiview video frame rate, the spatial resolution, the encoding quality and the number of views available in the teleimmersion. In this paper, because there is no temporal coding dependencies across the 3D multiview video frames, we simplify the problem by only focusing on the multiview video frame rate $q$. A larger $e_{V}$ means a greater motion smoothness and hence a better video signal intelligibility. We reduce the teleimmersive sample space by assuming a fixed spatial resolution, encoding quality and view number in our study.

\section{- Synchronization Quality}

The audio and multiple multiview video streams can experience different end-to-end delays (EED) be- 
tween two distributed users. We assume that the set of time-correlated multiview video frames is synchronized before it is sent to the display renderer for the purpose of accurate multiview video presentation. Hence, we only investigate the impact of the resulting audio-visual synchronization skew $e_{S}$ on the human perception. We use $\mathrm{EED}_{V}$ to represent the duration between the time that a time-correlated multiview video frame set is synchronously captured at the camera, and the time that it is displayed on the screen. $\mathrm{EED}_{A}$ is used to denote the duration between the microphone and speaker for an audio frame. Hence, $\rho_{S}$ can be represented as:

$$
e_{S}=\mathrm{EED}_{V}-\mathrm{EED}_{A}
$$

Note that $e_{S}>0$ means the audio is ahead of video, and that $e_{S}<0$ means the audio is behind.

\section{- Interactive Quality}

In CONV, the perception of a user on the interactivity is impacted by the delayed response of the remote site. A user can become impatient when the response delay accumulates, and the remote person becomes more distant. Doubletalks [133] may be introduced at an extremely long delay, when the user begins to repeat his statement, assuming his previous words are dropped during the transmission. Hence, the interactivity attribute can be characterized by the response delay $\left(e_{D}\right)$, which is incurred by the EED of local media streams (denoted as $\overline{\mathrm{EED}}$ ) to the remote site, the duration required for the remote user to think of a response (i.e., human response delay (HRD) [133]), and EED of the remote streams traveling back to the local site. Fig. 5.3 shows the concept. Mathematically, $e_{D}$ that a local user experiences can be represented as:

$$
e_{D}=\overline{\mathrm{EED}}^{U 1 \rightarrow U 2}+\mathrm{HRD}^{U 2}+\overline{\mathrm{EED}}^{U 2 \rightarrow U 1}
$$

where $U 1$ and $U 2$ represent the local and remote users, and $\mathrm{HRD}^{U 2}$ is the $U 2$ 's HRD.

On the other hand, the interactivity attribute in COLL is mainly evaluated by the collaborative performance of the two participants involved in the task. Here, "collaborative" means that two participants are following each other to achieve a mutual goal. A person (called initiator) initiates a gesture, and the other person (called follower) must exactly follow at the same time (i.e., HRD $\approx 0$ ). The two roles can be swapped during the activity. Because of the bi-directional EEDs of the media streams between the two parties, the response delay $e_{D}$ that an initiator perceives can be described as the timing mismatch in the collaboration on his/her own rendering display (Fig. 5.4). In this case, $e_{D}$ can be formulated as:

$$
e_{D}=\overline{\mathrm{EED}}^{U 1 \rightarrow U 2}+\overline{\mathrm{EED}}^{U 2 \rightarrow U 1}
$$

Because $\mathrm{EED}_{A}$ and $\mathrm{EED}_{V}$ may be different between two sites, we follow ITU-T G.1070 [22] and give 


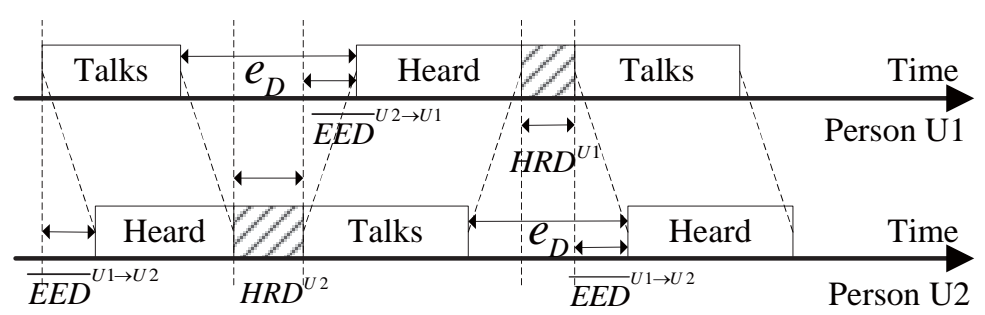

Figure 5.3: Interactivity in conversation-oriented activity.

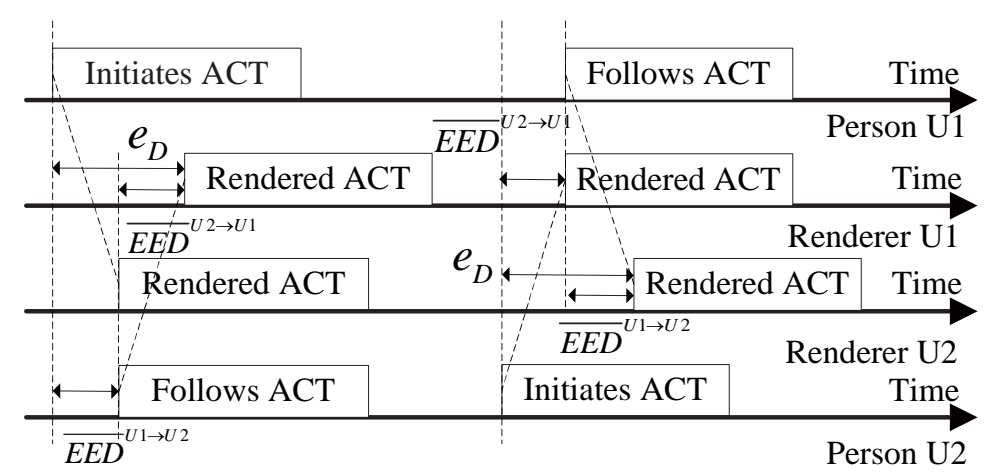

Figure 5.4: Interactivity in collaborative activity (ACT).

both metrics an equal weight in computing $\overline{\mathrm{EED}}$ in Eqn. 5.2 and 5.3, i.e.,

$$
\overline{\mathrm{EED}}=\left(\mathrm{EED}_{A}+\mathrm{EED}_{V}\right) / 2
$$

\section{- Combined Impacts}

The overall human subjective perception of teleimmersive shared activities are impacted by the combined impacts of the above user-observable quality attributes, which can be described by a 4-dimensional objective quality space with each objective streaming media quality point $\vec{e}$ in the space representing:

$$
\vec{e}=\left\{e_{V}, e_{A}, e_{D}, e_{S}\right\}
$$

In our user study, we create teleimmersive media samples with different configurations $\vec{e}$ (i.e., different values in one or multiple dimensions in $\vec{e}$ ). Throughout this chapter, we use frames per second (fps) for the unit of the multiview video frame rate $e_{V}$, milliseconds (ms) for the response delay $e_{D}$ and the audio-visual synchronization skew $e_{S}$, and $[1,4.5]$ for the audio quality $e_{A}$.

\subsubsection{Objective Metrics for Flicker Effect}

An objective streaming media quality point can change in two cases: (1) the network condition changes; and (2) the system adaptation moves its operating (quality) points. The flicker effect is incurred as the result 
of the perceptible change of a quality point. We formulate the flicker to capture this system behavior.

Definition 5.3.1 The flicker $f d\left(\vec{e}^{1}, \vec{e}^{2}\right)$ between two points $\vec{e}^{1}=\left\{e_{V}^{1}, e_{A}^{1}, e_{D}^{1}, e_{S}^{1}\right\}$ and $\vec{e}^{2}=\left\{e_{V}^{2}, e_{A}^{2}, e_{D}^{2}, e_{S}^{2}\right\}$ is:

$$
f d\left(\vec{e}^{1}, \vec{e}^{2}\right)=w_{V} \cdot\left|e_{V}^{1}-e_{V}^{2}\right|+w_{A} \cdot\left|e_{A}^{1}-e_{A}^{2}\right|+w_{D} \cdot\left|e_{D}^{1}-e_{D}^{2}\right|+w_{S} \cdot\left|e_{S}^{1}-e_{S}^{2}\right|
$$

where $w_{V}, w_{A}, w_{D}$ and $w_{S}$ are the normalized weights.

\subsection{Description of User Study}

Based on the above discussions and the subjective metrics in Section 2, we present the configurations of our user study in assessing the subjective quality of two teleimmersive applications. A larger flicker generally creates a greater human discomfort. So in our study, we focus on the evaluation of the streaming media quality.

We employ CCR in our evaluation, and analyze the user perceptual feedback using the comparative mean-opinion-score (CMOS), as discussed in Section 2.2. Our goal is to find the mapping from the fourdimensional objective quality metrics of the streaming media quality to the overall subjective human satisfaction $Q S$.

$$
\vec{e}=\left\{e_{V}, e_{A}, e_{D}, e_{S}\right\} \mapsto Q S(\vec{e})
$$

\subsubsection{Methodology}

Our user study investigates both teleimmersive shared applications: CONV and COLL. We will show both effectiveness and limitation of the subjective metric CMOS. To find the mapping from the objective quality metrics (Section 5.2) to subjective space (Section 2.2), we create teleimmersive media samples with different configurations $\vec{e}=\left\{e_{V}, e_{A}, e_{D}, e_{S}\right\}$ (Eqn. 5.5). However, the value of $\vec{e}$ can be continuously changing in its four-dimensional space, and thus, there can be infinite number of options. In this study, we discretize each metric within $\vec{e}$ (Table 5.1) according to the characteristics of real media traffic in the Internet.

In our user study, we ask the participants to compare teleimmersive media samples of the same activity (application) in each test. We employ CCR rating scale. We divide our tests into two categories, and process the user subjective feedback accordingly.

Category I: we only consider the impact of a single quality dimension in $\vec{e}$ by keeping values in other dimensions fixed. The diversity of user votes is expected to be small, and mutually contradicting votes are unlikely. So we focus on presenting the CMOS effectiveness. 
Table 5.1: Discretization of quality metrics in $\vec{e} . \mathrm{HRD}=800 \mathrm{~ms}$ is used in computing $e_{D}$ in CONV (Section 3.2). $e_{V}$ is rounded to the nearest integer in the evaluation.

\begin{tabular}{|c|c|c|}
\hline Metric & Unit & Discretiziation \\
\hline \hline$e_{V}$ & fps & $2.5,5,7.5,10,12.5,15,17.5,20$ \\
\hline$e_{A}$ & {$[1,4.5]$} & $2.0,4.0$ \\
\hline$e_{S}$ & $\mathrm{~ms}$ & $0, \pm 75, \pm 150, \pm 225$ \\
\hline $\begin{array}{c}e_{D} \\
(\mathrm{CONV})\end{array}$ & $\mathrm{ms}$ & $\begin{array}{c}1000,1200,1400,1600, \\
1800,2000,2200,2400,2600\end{array}$ \\
\hline $\begin{array}{c}e_{D} \\
(\mathrm{COLL})\end{array}$ & $\mathrm{ms}$ & $\begin{array}{c}120,180,240,300, \\
360,420,480,540,600\end{array}$ \\
\hline
\end{tabular}

Category II: we compare teleimmersive media samples with quality tradeoffs, and show the diversity of user opinions. In this case, we will discuss CMOS, the distribution of the user votes $\left(n_{>0}, n_{=0}, n_{<0}\right)$, i.e., the number of positive, zero and negative CCR votes and the resulting dominant opinion, as presented in Section 2.2. We will show the limitation of CMOS by identifying the inconclusiveness of the subjective comparisons in the study.

\subsubsection{Preparation of Teleimmersive Media Samples}

We let two participants be situated at different sites and conduct activities through the teleimmersive system. The two sites are in the same local area network (LAN), so the outputs should be assumed to have no video and audio signal degradation with minimal latency and perfect synchronization. We record the distortionfree audio and video at both sites. For the video, because the teleimmersive system eventually displays the multiview images on the $2 \mathrm{D}$ screen, we record the $2 \mathrm{D}$ video including both participants which is exactly shown on the screen (using the xvideocap software ${ }^{1}$ ) instead of the original multiview images. For the audio, we mix the audio talkspurts of the two parties (using the Virtual Audio Cable software ${ }^{2}$ ), and xvideocap can also be utilized to record the mixed audio, with an automatic synchronization with the video.

We create teleimmersive media samples for both CONV and COLL applications. In CONV, we follow our previous VoIP study and use a HRD of $800 \mathrm{~ms}$, and an average talkspurt duration of $2732 \mathrm{~ms}$ in our simulation [133]. In COLL, the average duration of talkspurts is $856 \mathrm{~ms}$. In this study, the reference sample with the best-possible (called optimal in this paper) quality, assuming two sites are communicating in LAN, is $\vec{e}^{*}=\{20,4.0,800,0\}$ for CONV, or $\vec{e}^{*}=\{20,4.0,0,0\}$ for COLL.

We now assume that one teleimmersive site is local and the other is remote. We introduce the delay and

\footnotetext{
${ }^{1}$ http://xvidcap.sourceforge.net

${ }^{2}$ http: //software.muzychenko. net/eng/vac.htm
} 
synchronization skews for the remote streams, and impose degradations on its media signal intelligibility (reduced $e_{V}$ and $e_{A}$ ). The qualities of local audio and images remain untouched. The degraded teleimmersive media sample $\vec{e}$ describes the objective quality of the remote streams.

\subsubsection{Setup of User Study}

19 participants (average age: 26) are involved in our user study, and are trained to use CCR scales consistently before the subjective tests. They are required to sit 1.5 meter apart from a 61 -inch NEC screen (resolution: 1280x720), and to rate teleimmersive media samples at different $\vec{e}$ values. The video is rendered at a resized resolution of 640x360 (original resolution: 420x240). The audio is played at a DELL AY410 2.1 speaker. To simulate a real teleimmersive media involvement, these observers are told to be assuming themselves sitting closely to the person in the local site, so they can pay more attentions to the (degraded) quality of the remote person.

A total of 240 comparisons of teleimmersive media samples (with different configurations $\vec{e}$ ) is conducted within the whole test. Participants are able to pause at any time throughout the test. There are 10-second idle pauses between two consecutive comparisons, in order to allow the observers to have sufficient time to consider their votes.

\subsubsection{Subjective Evaluation Results}

We present the findings of both test categories from our user study. We focus on the effectiveness (conclusiveness) of CMOS in Category I, while addressing the limitation (inconclusiveness) of the CMOS metric in Category II. We will show the two applications CONV and COLL have heterogeneous impacts on the human perception. Based on the subjective findings, we will discuss their implications to the control design, and conclude with a need for a new subjective metric to describe the inconclusive comparisons.

\section{- Category I: Audio Signal Intelligibility}

The audio PESQ (i.e., $e_{A}$ ), as its name suggests, is computed on a psycho-acoustic scale which is already able to describe the real human subjective perception on audio signals. That is to say, when we fix $q, e_{D}$ and $e_{S}$ as optimal, we are able to approximate the impairment of $e_{A}$ as:

$$
\operatorname{CMOS}\left(e_{A}\right)=4.5-e_{A}
$$

Here, 4.5 is the maximal-possible value of $e_{A}$. Our CCR findings are aligned with the PESQ results, 

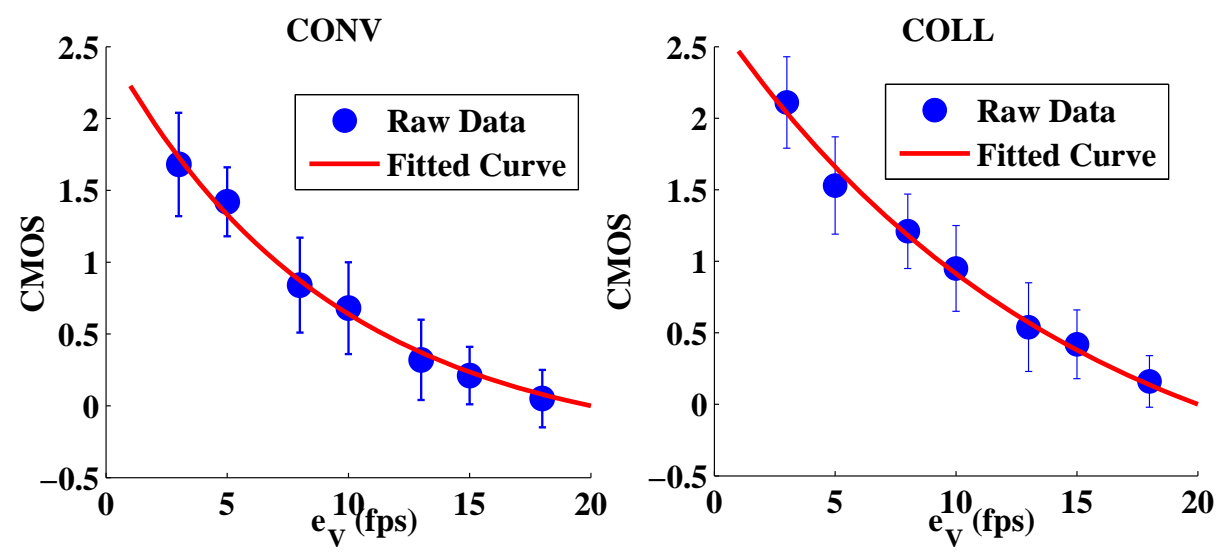

Figure 5.5: CMOS and 95\% confidence intervals comparing optimal reference $\vec{e}^{*}$ (first sample in the comparison) and $e_{V}$-degraded samples (but optimal $e_{A}, e_{D}, e_{S}$ ).

thus showing the CCR effectiveness.

\section{- Category I: Video Signal Intelligibility}

Fig. 5.5 shows the CMOS results comparing $\vec{e}^{*}$ to the samples with different degraded $e_{V}$ while keeping other quality dimensions optimal. We modify the exponential model in [134] to find the fitting curve describing the mapping from $e_{V}$ to the corresponding CMOS:

$$
\operatorname{CMOS}\left(e_{V}\right)=Q-Q \times \frac{1-e x p^{-c \times e_{V} / e_{V}^{\max }}}{1-e x p^{-c}}
$$

In this equation, $c$ is the slope of the curve, which describes the impact of $q_{V}$ changes on the CMOS. A smaller $c$ will introduce a larger degradation to CMOS at the same $e_{V} . Q$ represents the maximum-possible

impairment of $e_{V} \cdot e_{V}^{\max }$ is set to be $20 \mathrm{fps}$, the maximum multiview video frame rate in our study. We want to find the best fitting parameters $Q$ and $c$ of the exponential curve. We utilize the nonlinear fitting tool in Matlab (nlinfit function) to compute $Q$ and $c$. The fitting results as well as the corresponding mean squared error (MSE) are shown in Table 5.2. Because $c$ is smaller in COLL, an equal $e_{V}$ decrease can cause greater perceptual degradations in COLL than CONV. The reason is due to more frequent body movement in the COLL activity.

In both cases, the reasonable confidence interval lengths (within $\pm 0.2 \sim \pm 0.32$ ) show the CCR effectiveness.

\section{- Category I: Synchronization Impairment}

In Fig. 5.6 (1) and (3), we carry on experiments to evaluate the lip skew impairment at the optimal $q$, 
Table 5.2: Fitting results for Eqn. 5.9.

\begin{tabular}{|c|cc|c|}
\hline & $Q$ & $c$ & MSE \\
\hline \hline CONV & 2.52 & 2.16 & 0.01 \\
\hline COLL & 2.71 & 1.35 & 0.01 \\
\hline
\end{tabular}

(1) CONV (sole $e_{s}$ impact)

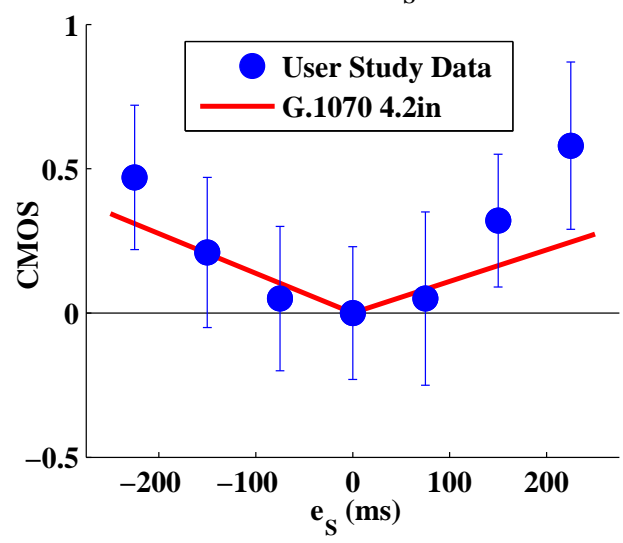

(3) COLL (sole $e_{s}$ impact)

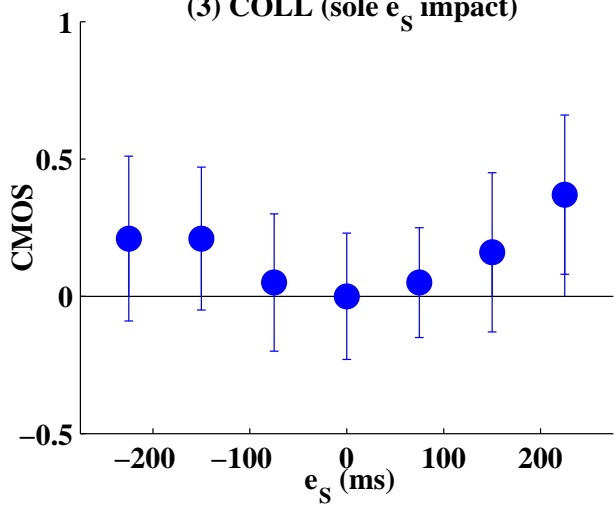

(2) $\operatorname{CONV}\left(e_{S}=150 \mathrm{~ms}\right)$

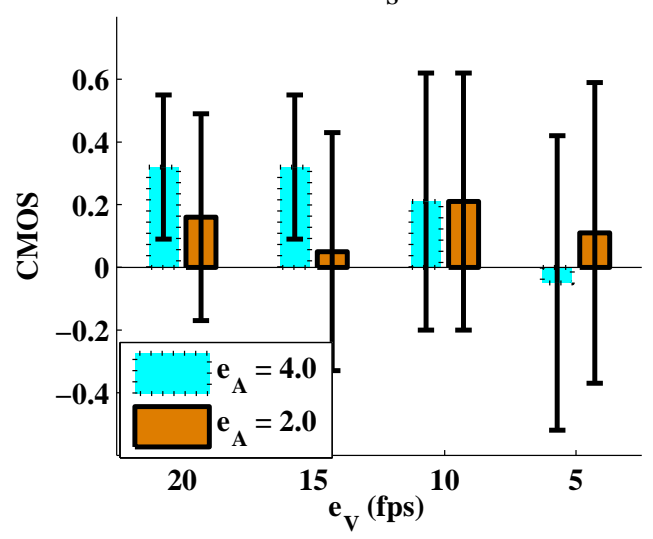

(4) $\operatorname{COLL}\left(e_{s}=-150 \mathrm{~ms}\right)$

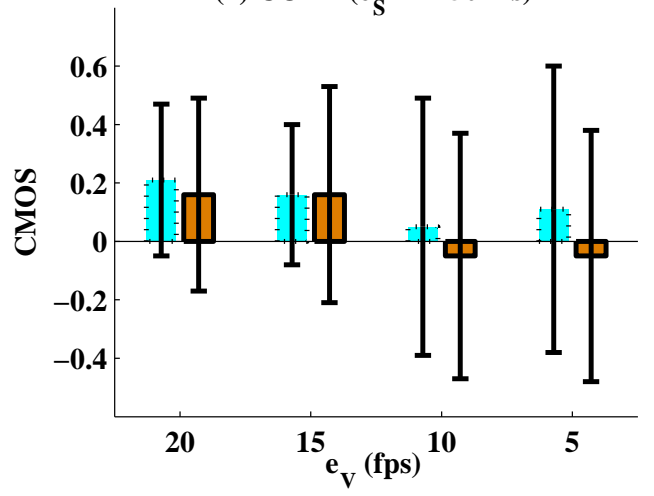

Figure 5.6: CMOS results and 95\% confidence intervals. (1) and (3) show the comparisons between the optimal reference $e^{*}$ (first sample in the comparison) and a sample with a degraded $e_{S}$ (but with the same optimal $\left.e_{V}, e_{A}, e_{D}\right)$. (2) and (4) show the two samples with different $e_{S}$, but same $e_{V}, e_{A}$, and $e_{D}=800 \mathrm{~ms}$ for CONV and $0 \mathrm{~ms}$ for COLL. The first sample in the comparison is $e_{S}=0$ and the second is $e_{S}= \pm 150$ ms. 
$e_{A}$ and $e_{D}$, when compared to $\vec{e}^{*}$. We show the CMOS results and $95 \%$ confidence intervals at different $e_{S}$. In Fig. 5.6 (2) and (4), we evaluate the impact of $e_{V}$ and $e_{A}$ on the synchronization quality. Due to the space limit, we only show the results at selected $e_{S}$ values ( $e_{S}=150 \mathrm{~ms}$ for CONV and $-150 \mathrm{~ms}$ for COLL) with different $e_{V}$ and $e_{A}$ options, compared to the samples of $e_{S}=0$ with the same media signal intelligibility. We have three observations.

First, our limited study reflects that the heterogeneous teleimmersive activities can affect the synchronization perfection. Generally, the degradation of a lip skew in the COLL environment is smaller than that in CONV with the same skew, because (a) the talkspurt durations in COLL are much shorter, and (b) people are focusing on the visual collaborative activity more than talkspurts in COLL. The lengths of confidence intervals are comparable in the two applications.

Second, our study exhibits that people are more tolerant of video ahead of audio $\left(e_{S}<0\right)$ than audio ahead of video $\left(e_{S}>0\right)$. The reason is that the talkspurt durations in teleimmersive shared activities are generally much shorter than those in on-demand videos, so a lip skew at the end of an utterance is more noticeable. Fig. 5.6 shows that a late video portion at the time that an utterance has been fully played has a greater perceptual impact than a late audio portion.

Third, Fig. 5.6 (2) and (4) show that both $e_{V}$ and $e_{A}$ do impact the synchronization quality. We find that the lengths of confidence intervals are much larger $(> \pm 0.4)$ as $Q_{V}$ and $e_{A}$ degrade (e.g., $e_{V}=5$ fps or $\left.e_{A}=2.0\right)$. When the multiview video frame rate lowers, the motion jerkiness becomes the dominant factor degrading the human perception, and thus, a lip skew can be difficult to tell. On the other hand, when $e_{A}$ is small, the poor audio intelligibility also creates a hard time for users to differentiate a lip skew, and an incomplete utterance can cause misperception on the synchronization quality.

\section{- Category I: Interactive Quality}

We conduct tests for evaluating the $e_{D}$ impairment. These include two sets of comparisons. In the first set, we study the sole $e_{D}$ impact at the optimal $e_{V}, e_{A}$ and $e_{S}$. We show the corresponding CMOS and confidence intervals by referencing $e^{*}$ in Fig. 5.7 (1) and (3). The G.107 and G.1070 findings are also plotted in CONV as a comparison. In the second set, we study the effects of the media signal intelligibility on the $e_{D}$ perception. Due to space limit, Fig. 5.7 (2) and (4) only show the results for selected $e_{D}$ values. There are several observations.

First, we follow [39] and use a third-order polynomial model to describe the CMOS degradations due to $e_{D}$. The results are shown in Fig. 5.7 (1) and (3). 

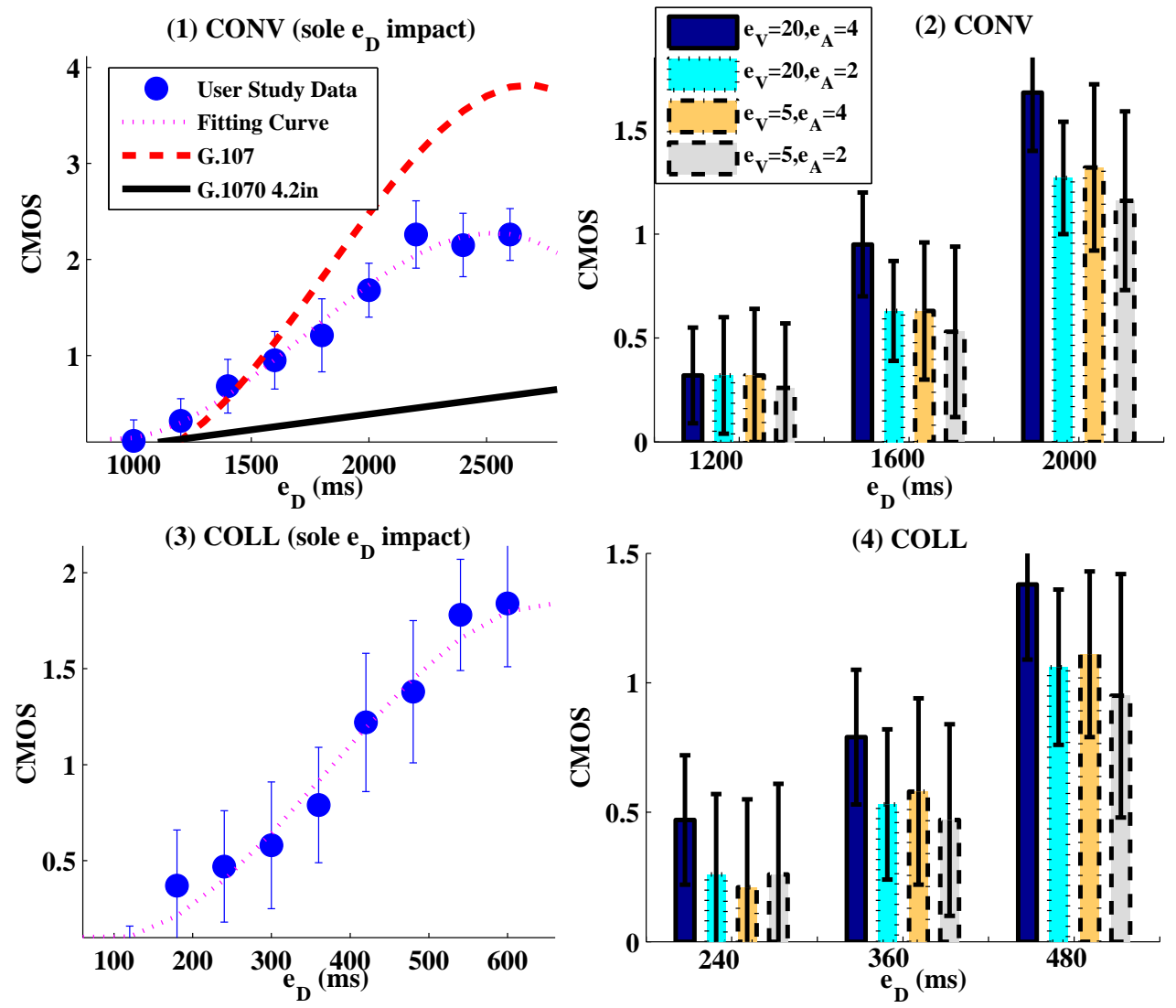

Figure 5.7: CMOS results and 95\% confidence intervals. (1) and (3) show the comparisons between the optimal reference $\vec{e}^{*}$ (first sample in the comparison) and a sample with a degraded $e_{D}$ (but with the same optimal $e_{V}, e_{A}$ and $e_{S}$ ). G.107 and G.1070 delay curves are also drawn in (1). (2) and (4) show the results for two samples with different $e_{D}$, but same $e_{V}, e_{A}$, and $e_{S}=0$. The first sample in the comparison is $e_{D}=$ $800 \mathrm{~ms}$ for CONV and $0 \mathrm{~ms}$ for COLL, and the second is with the degraded $e_{D}$ as indicated in the figures. 
Table 5.3: Fitting results for Eqn. 5.10.

\begin{tabular}{|c|cccc|c|}
\hline & $a_{3}$ & $a_{2}$ & $a_{1}$ & $a_{0}$ & MSE \\
\hline \hline CONV & $1.033^{-9}$ & $5.342^{-6}$ & -0.007 & 3.036 & 0.010 \\
\hline COLL & $-1.945^{-8}$ & $2.163^{-5}$ & -0.003 & 0.231 & 0.009 \\
\hline
\end{tabular}

$$
\operatorname{CMOS}\left(e_{D}\right)=a_{0}+a_{1} \cdot e_{D}+a 2 \cdot e_{D}^{2}+a_{3} \cdot e_{D}^{3}
$$

Table 5.3 presents the fitting results for both activities as well as the corresponding MSE. Generally for CONV, $e_{D}<1200 \mathrm{~ms}$ is desired (CMOS $\left.<0.5\right)$ and $e_{D}>2000 \mathrm{~ms}$ is bad (CMOS $>1.5$ ). For COLL, $e_{D}<200 \mathrm{~ms}$ is desired $(\mathrm{CMOS}<0.5)$ and $e_{D}>400 \mathrm{~ms}$ is bad (CMOS $\left.>1.5\right)$. Hence, the COLL application requires a higher demand for interactive quality than CONV. This is because people in COLL attach more importance to the visual timing mismatch in the collaboration. The derived curves prove the CCR effectiveness in describing human perception.

Second, we find that our CONV findings are in between the G.107 and G.1070 delay curves. The reason is that a user in a VoIP application (G.107) usually lacks a perception of the activities of the remote party. So the local person is prone to assuming the remote talkspurts have been dropped by the Internet at a delayed response, and may repeat his/her utterances which can cause doubletalks. On the other hand, a person in either a video conferencing (G.1070) or a teleimmersive session is able to see what the remote user is doing, and hence he/she is more tolerant of the delay. But in teleimmersive shared activities, because both people are located in an immersive environment, a higher demand for interactive quality is expected, compared to the video conferencing. In addition, the delay results that G.1070 obtains are somewhat too conservative.

Third, we demonstrate that the media signal intelligibility does affect the interactivity perception, as in Fig. 5.7 (2) and (4). The figures show that, a delayed response has less impacts on human perceptual degradations (smaller CMOS in the figures) in an environment with reduced video motion smoothness and audio signal intelligibility.

\section{- Category II}

In this study, we have done substantial subjective comparisons over the teleimmersive media samples with multi-dimensional quality tradeoffs. We focus on the tradeoff between $q_{V}$ and $e_{D}$, which is most commonly seen in the real teleimmersive systems over the Internet. The reason is that the data rate for multiview videos is very high. By increasing $e_{V}$ at a fixed bandwidth availability, additional transmission time will be introduced over the Internet and end systems, which in turn degrades (increases) $\oplus_{D}$. Fig. 5.8 
and 5.9 show some of the selected representative results, where both $e_{A}=4.0$ and $e_{S}=0$ are fixed. We compute the distribution of user votes $\left(n_{>0}, n_{=0}, n_{<0}\right)$, as well as the corresponding CMOS. Several observations are to be noted.

First, we find a huge diversity of user votes in some of the comparisons (e.g. Fig. 5.8(6) in CONV and Fig. 5.9(4) in COLL). The multi-dimensional quality tradeoff contributes to this diversity. Generally, if the perceptual degradation in one quality dimension of a teleimmersive media sample is not overshadowed by the enhancement of another dimension, users can output contradicting voting scores, because they can attach heterogeneous importance to different quality attributes based on their individual interests. For example, in Fig. 5.8(6), 9 out of 19 participants prefer a better interactive quality, so they think the first sample is better. Another 9 participants like a smoother body motion in the video, so they argue for the second sample. As two quality points are moving apart on the tradeoff curve, and one one dimension is gradually improving as another dimension is worsening comparably, the likelihood of outputting contradicting opinions is increasing (e.g., Fig. 5.8(6) shows a greater voting diversity than Fig. 5.8(3) and (5)).

Second, the interpretation of the average score CMOS may lack the statistical significance at a large variance of user votes. For example, a CMOS $=0$ in Fig. 5.8(6) cannot tell with confidence whether a sample within a comparison is of the same quality with the other sample (actually the qualities of the two samples in Fig. 5.8(6) are completely different).

To evaluate the inconclusiveness, we use $\alpha=90 \%$ significance in Eqn. 2.10. Because $N_{\text {total }}=19$, this returns $N_{\text {th }}=13$. Hence, except Fig. 5.8(1) which leads to a dominant opinion that the first sample is "better" than the second one. All other comparisons are "inconclusive" (i.e., no dominant opinion).

\subsubsection{Remarks}

The above discussions imply two important aspects in designing an interactive teleimmersive system.

Teleimmersive activity (application) heterogeneity. A good media system should not only be able to adapt to Internet dynamics, but also be built upon the heterogeneous characteristics of teleimmersive activities (applications) to meet the real user demands. From our study, we qualitatively conclude the perceptual importance for the two teleimmersive applications in Table 5.4. Compared to COLL, CONV generally requires a higher demand for the audio signal intelligibility and the constrained lip skew, but a lower expectation on the video motion smoothness and interactive quality. These characteristics should be addressed in the system design.

Ordering of subjective scores. Previous studies on VoIP or video conferencing $[39,170]$ usually 
(1) $(5,1200)$ vs $(20,1600)$

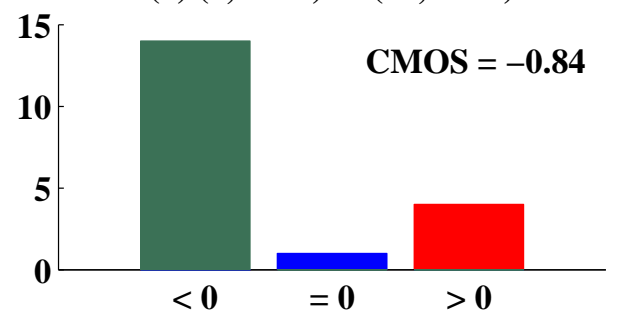

(3) $(5,1200)$ vs $(10,1600)$

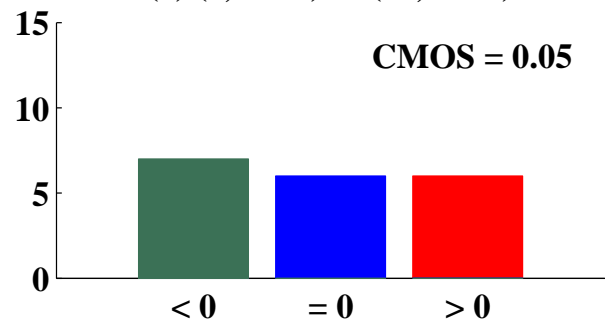

(5) $(10,1600)$ vs $(20,2000)$

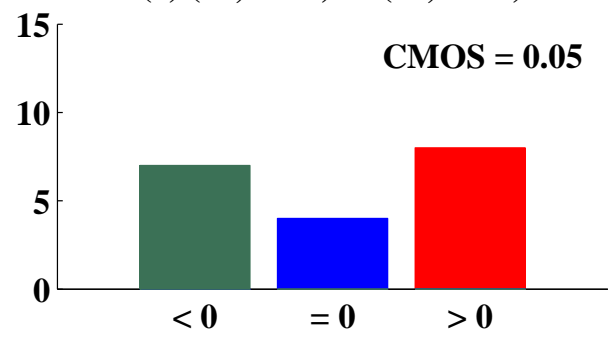

(2) $(5,1200)$ vs $(15,1600)$

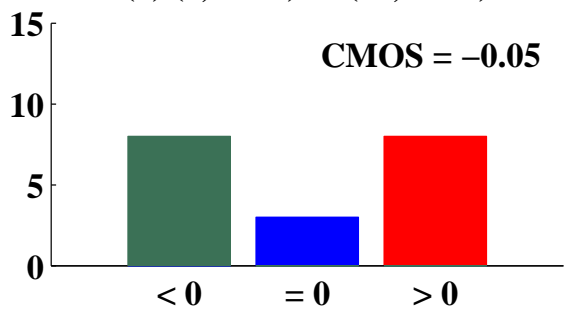

(4) $(15,1600)$ vs $(20,2000)$

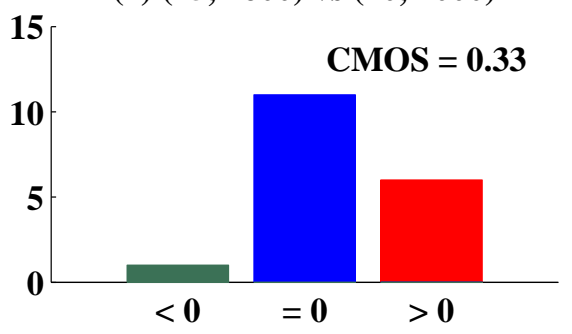

(6) $(5,1200)$ vs $(20,2000)$

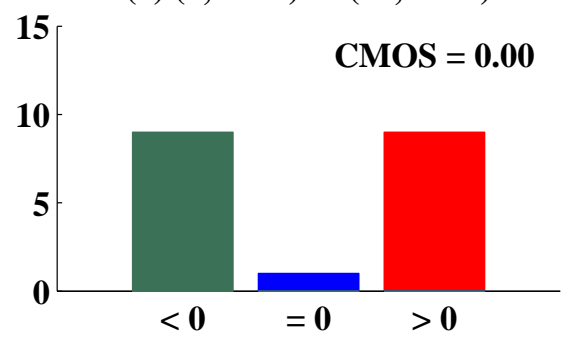

Figure 5.8: Comparison results $\left(N_{>0}, N_{=0}, N_{<0}\right)$ for samples in CONV. All samples with $e_{A}=4.0$ and $e_{S}=0$. The caption format is $\left(e_{V}^{1}, e_{D}^{1}\right)$ vs $\left(e_{V}^{2}, e_{D}^{2}\right)$, where the four numbers are the $e_{V}$ and $e_{D}$ of the first and second samples. 
(1) $(5,240)$ vs $(20,360)$

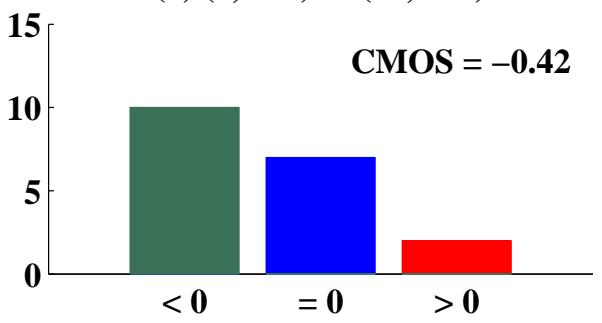

(3) $(5,240)$ vs $(10,360)$

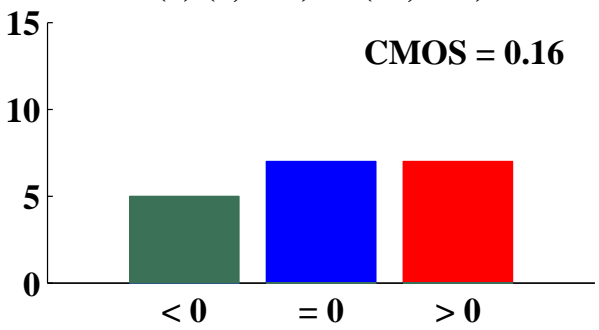

(5) $(10,360)$ vs $(15,480)$

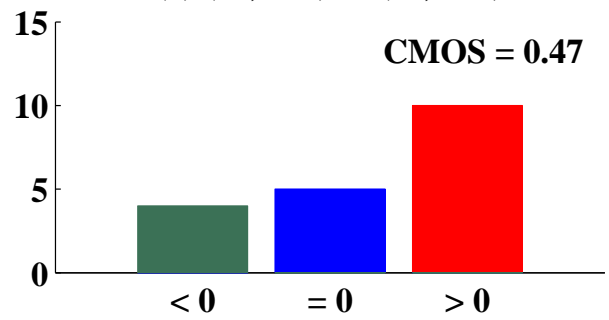

(2) $(5,240)$ vs $(15,360)$

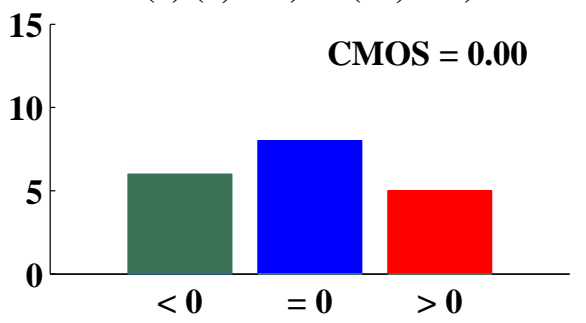

(4) $(10,360)$ vs $(20,480)$

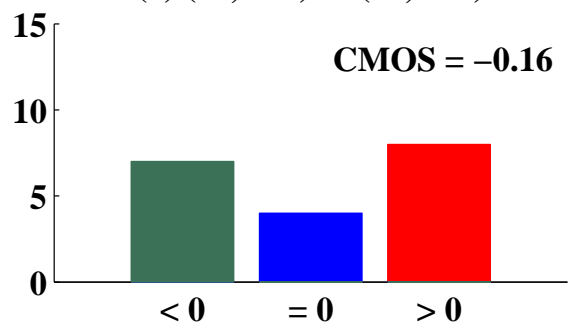

(6) $(5,240)$ vs $(20,480)$

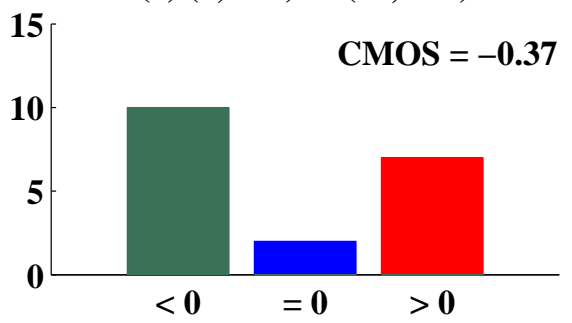

Figure 5.9: Comparison results $\left(N_{>0}, N_{=0}, N_{<0}\right)$ for samples in COLL. All samples with $e_{A}=4.0$ and $e_{S}=0$. The caption format is $\left(e_{V}^{1}, e_{D}^{1}\right)$ vs $\left(e_{V}^{2}, e_{D}^{2}\right)$, where the four numbers are the $e_{V}$ and $e_{D}$ of the first and second samples. 
Table 5.4: Comparisons for CONV and COLL characteristics. Note that H/L mean comparatively more/less important between the two application.

\begin{tabular}{|c|cccc|}
\hline & $e_{V}$ & $e_{A}$ & $e_{D}$ & $e_{S}$ \\
\hline \hline CONV & $\mathrm{L}$ & $\mathrm{H}$ & $\mathrm{L}$ & $\mathrm{H}$ \\
\hline COLL & $\mathrm{H}$ & $\mathrm{L}$ & $\mathrm{H}$ & $\mathrm{L}$ \\
\hline
\end{tabular}

propose adaptation algorithms based on the the (extended) quality models used in G.107 and G.1070. Here, we argue that the quality closed forms derived in both standards are only suitable for subjective quality assessment of media samples. The resulting score orderings, however, are not good for system adaptations.

First, multiple quality points, which are distant in the multidimensional Euclidean space, can lead to same or similar CMOS when they are compared to the optimal reference $\vec{e}^{*}$. For example in CONV, $\vec{e}^{1}=\{12,4.0,0,0\}$ in Fig. 5.5(1), $\vec{e}^{2}=\{20,4.0,0,-225\}$ in Fig. 5.6(1), and $\vec{e}^{3}=\{20,4.0,1300,0\}$ in Fig. 5.7(1) all lead to CMOS of around 0.5. If we achieve synchronization control adaptation based on the score ordering, we may switch between two operating configurations $\vec{e}$, which are close in the subjective score space, but are actually distant in terms of each objective quality dimension. This can cause flicker effects. The quality flickers should be minimized, which would otherwise downgrade the human perception.

Second, as we have discussed in Category II tests, the diversity of user votes under the tradeoffs of multiple quality dimensions, make it difficult to interpret the obtained CMOS scores with statistical significance. Because the comparison results can lead to an "inconclusive" opinion, a total ordering of multiple quality points may not be accessed, and only a partial order can be decided. Similar conclusions have also been reached in our previous VoIP studies [36, 37, 133].

A need for new subjective metric. Given the inconclusiveness of CMOS at the diversity of user voting scores, we conclude that there is a need to propose a new subjective metric to interpret the inconclusive subjective results under multi-dimensional quality tradeoffs, which will be discussed in Section 5.5.

\subsection{Perception-driven Presentation Scheduling (MPS) for Multimedia Synchronization}

We start with the proposal of the new subjective metric called preference to capture the comparison inconclusiveness. After that, we evaluate the interaction of the MPS algorithm, with its resulting human perceptual impact on both streaming media quality and the flicker degradation. Based on our proposed preference metric and the generalized subjective evaluation results using the support vector machine (SVM) [37, 41] 
(details will not be discussed), we will then present the perception-driven MPS for real-time synchronization of the teleimmersive applications.

\subsubsection{New Subjective Metric: Preference}

The preference metric is defined as follows:

Definition 5.5.1 A user preference is either aligned with the dominant opinion, or with the "inconclusive" comparison.

Example 5.5.1 If the dominant opinion is the media sample 1 is "better" than the sample 2, the resulting preference is "better". If the test does not conclude any dominant opinion with statistical significance, the user preference is "inconclusive".

Note that, while the preference metric is able to describe the inconclusive results, it is unable to interpret the level of each opinion (i.e., the quality difference of two media samples) under a conclusive comparison, which is what CMOS is meant to achieve. In our synchronization control adaptations, because we are only interested in knowing which operating status is better, or if two statuses cannot lead to a conclusive answer, the preference metric well serves the demand of our study.

\subsubsection{Interactions between MPS and Human Perception}

\section{- MPS and Tradeoffs of Streaming Media Quality}

The receiver buffer size decides the EED of multimedia data and schedules their presentation at the output devices. Both $e_{V}$ and $e_{A}$ depend on the receiver video and audio buffer size, and thus, the corresponding MPS-controllable EED values: $\mathrm{EED}_{V}$ and $\mathrm{EED}_{A}$. Because larger delay fluctuations can be smoothed as the result of a prolonged receiver buffer size, $e_{V}$ and $e_{A}$ are improved as $\mathrm{EED}_{V}$ and $\mathrm{EED}_{A}$ increase. But this will increase (degrade) $e_{D}$ and impact $e_{S}$ (Fig. 5.10). A mapping can be found between $\mathrm{EED}_{V}$ and $\mathrm{EED}_{A}$, and the objective quality space, given a network condition NC:

$$
\text { Given NC : }\left\{\mathrm{EED}_{V}, \mathrm{EED}_{A}\right\} \mapsto \vec{e}=\left\{e_{V}, e_{A}, e_{D}, e_{S}\right\}
$$

Because the mapping from the MPS control variables $\mathrm{EED}_{V}$ and $\mathrm{EED}_{A}$ to the objective quality space $\vec{e}$ can be accessed online, objective quality metrics $\left\{e_{V}, e_{A}, e_{D}, e_{S}\right\}$ are usually employed for real-time MPS control. Due to a lack of closed form describing their tradeoffs and combined impacts, a MPS scheme 


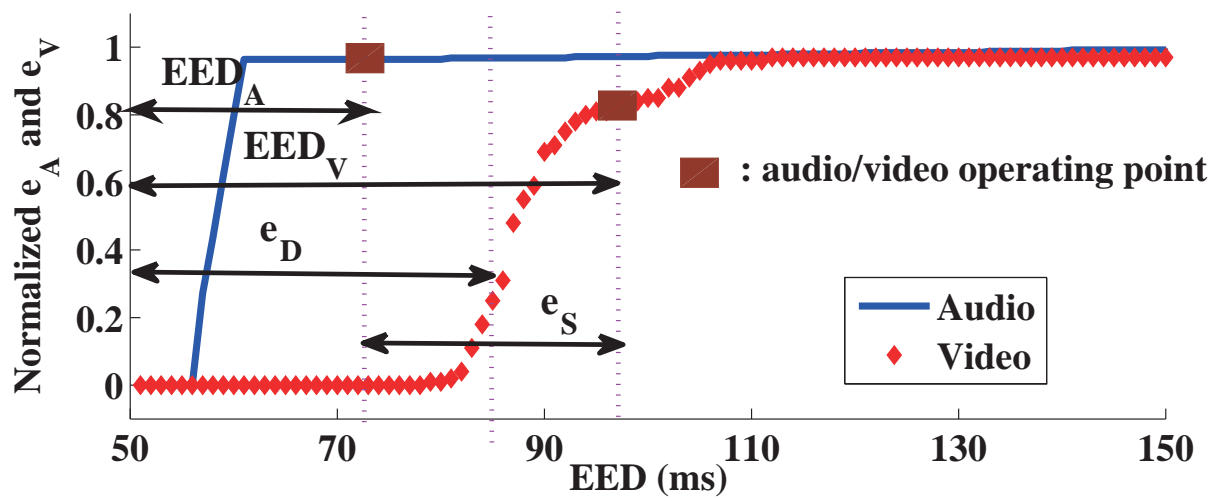

Figure 5.10: Tradeoffs among objective metrics of the teleimmersive streaming media quality.

is unable to find a best operating (quality) point leading to the optimal overall perceptual quality without the aid of offline subjective evaluation results, which we have obtained in Section 5.4.

\section{- Design Rationale of Perception-driven MPS}

Given the mapping from the MPS control space to the objective streaming media quality space (Eqn. 5.11), and from the objective streaming media quality space to the subjective user satisfaction (Eqn. 5.7), we now show the interaction between the MPS control variables and the resulting subjective/objective quality metrics, as outlined in Fig. 5.11.

The MPS control variables can only achieve the adaptations by employing objective streaming media quality metrics serving as direct online performance indicators of different quality attributes. But due to the tradeoffs among these objective metrics, only a set of non-dominated Pareto optimal (multi-dimensional objective quality) points can be obtained. For each non-dominated Pareto optimal point, we cannot improve the (objective quality) value of one of its dimensions without worsening the values of others.

Because these objective quality points cannot describe the real user satisfactions, we will rely on our subjective evaluation results for selecting the perceptual optimum among the Pareto optimal points online. We employ the proposed subjective preference metric in the offline user study due to its capability to capture the user opinion diversity. According to Section 4.4, two objective quality points can be mutually "inconclusive". Thus, a partial order of these points may lead to the fact that we are unable to find a global perceptual optimum among these objective quality points, but rather several points which are locally perceptual optimal, and thus, are perceptually no "worse" than each other (i.e., mutually "same" or "inconclusive").

Among these local perceptual optima, different candidates can result in heterogeneous flicker impairment due to the changes of both control statuses and Internet quality. To minimize the human perceptual degradation, we will select among these local optima with the minimal flicker degradation, as the best online 


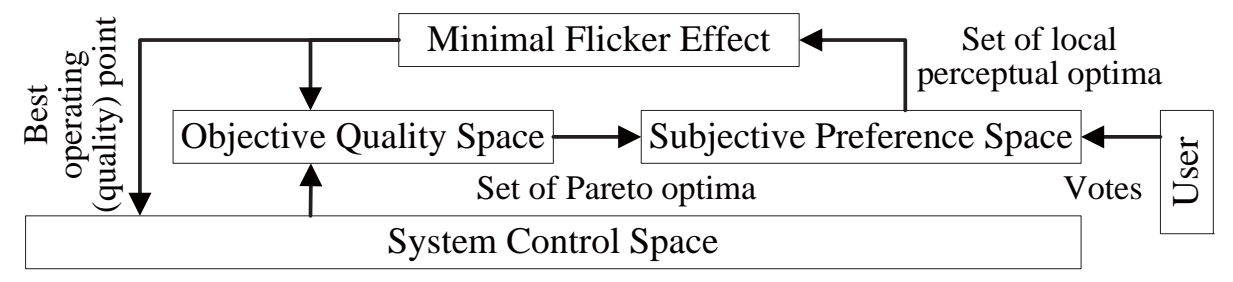

Figure 5.11: Interaction of quality metrics with control variables

operating point.

\subsubsection{Design of Perception-driven MPS}

Here, we present a new perception-driven MPS scheme for multimedia synchronization that adapts the objective quality operating points under Internet dynamics, and consistently deliver high subjective media bundle perception with minimal flicker effects. At each MPS control update, the selection of a new operating objective quality point expects the following three goals.

1. The candidate point must be decided dynamically based upon the recent (short-term) Internet conditions.

2. Because the network condition may change between two consecutive MPS control updates, the candidate should also be robust to Internet dynamics, in the sense that we prefer a candidate (and its corresponding control values $\left\{\mathrm{EED}_{V}, \mathrm{EED}_{A}\right\}$ ) that will introduce a smaller flicker due to any potential Internet condition variations (based on long-term Internet observations).

3. The receiver control prefers a new operating point closer to the point immediately before the control update so that the flicker effect due to the system adaptations can be reduced.

Hence, the overall MPS scheme can be divided into the corresponding three steps.

\section{- Step 1: Searching Local Perceptual Optima}

The presentation control updates the local perceptual optima according to the most recent short-term Internet conditions, by finding the tradeoffs among the objective quality points and employing human preference results to select among the resulting Pareto optima. The difficulty, however, is that there are infinite number of objective quality points on the operating plane, so searching over the entire plane can be costly. Here, we propose a perception-based genetic approach to address the issue. 
The tradeoffs among multiple objective quality metrics in the continuous space can be formulated as a multi-objective optimization problem. The genetic algorithm has been proven successful in locating Pareto optimal points efficiently. In our study, we modify the original genetic algorithm and incorporate the subjective preference metric, so that the new algorithm is able to search the local perceptual optima.

In the teleimmersive application, each objective quality metric within $\vec{e}=\left\{q_{V}, e_{A}, e_{D}, e_{S}\right\}$ can be regarded as an objective function in the multi-objective optimization formulation, and $\vec{g}=\left\{\mathrm{EED}, \mathrm{EED}_{A}\right\}$ is the input control vector. Based on the Internet statistics within the most recent (short-term) timing window, we are able to find the mapping from the control vector to the objective metrics. We utilize the genetic NSGA-II implementation [171] for its computation efficiency. The key idea of the genetic approach is that in each iteration, it generates new populations (i.e., a set of $\vec{g}=\left\{\mathrm{EED}_{V}, \mathrm{EED}_{A}\right\}$ at different values) using the standard crossover/mutation methods. It then prioritizes the solutions based on their fitness values (defined below), and selects those with better fitness as the populations in the next iteration. We make modifications to NSGA-II to incorporate the human subjective preferences.

First, we redesign the fitness value of the solutions based on two factors. (1) Original Pareto ranking in NSGA-II [171]. For each solution $\vec{g}^{i}$ in the current iteration, we compute the number of other solutions in the populations that dominate this solution, and denote it as $h(\vec{g})$. A solution $\vec{g}^{1}$ whose $h\left(\vec{g}^{1}\right)=0$ means a non-dominated solution. (2) Subjective preference status. A non-dominated solution that is perceptually no "worse" (in terms of the preference metric) than any other solution up till the current iteration, denoted as $\vec{g}$, is given the top priority. Here, subjective comparisons are conducted between the corresponding objective quality points of the solutions. We set the fitness value of $\vec{g}^{2}$ to $h\left(\vec{g}^{2}\right)=-1$. We call $\vec{g}^{2}$ as a perceptual elitist. Hence, in our algorithm, a solution with a smaller fitness value represents a better solution.

Second, we maintain the complete list of perceptual elitists, which is always used as a part of the populations of the next iteration. In each iteration, a solution in the elitist list is removed from the list if (a) it is dominated by other solutions in the population, or (b) it is perceptually "worse" than a new nondominated solution. Note that multiple mutually "same" solutions can be crowded together. To maintain the diversity within the elitist list, we prescribe that for any two solutions in the list, their Euclidean distance should not be less than a minimal threshold $\delta g(=50 \mathrm{~ms}$ in our evaluations).

Example 5.5.2 We suppose $\vec{g}^{2}$ is originally in the perceptual elitist, and we have three new non-dominated solutions: $\vec{g}^{1}, \vec{g}^{0}$ and $\vec{g}^{3}$. Because $\vec{g}^{1}$ dominates $\vec{g}^{2}$, we remove $\vec{g}^{2}$ from the elitist list, and add $\vec{g}^{1}$. We then compare $\vec{g}^{1}$ and $\vec{g}^{0}$ by the subjective preference, and the result shows that $\vec{g}$ is perceptually "better" than $\vec{g}^{1}$. So we replace $\vec{g}^{1}$ with $\vec{g}^{0}$ in the elitist list. Now the comparison between $\vec{g}^{0}$ and $\vec{g}^{3}$ is "same". But the 
Euclidean distance between $\vec{g}^{0}$ and $\vec{g}^{3}$ is smaller than $\delta g$, so we will not add $\vec{g}^{3}$ to the list.

Our online perception-based genetic algorithm terminates beyond certain preset timing constraints. It will return solutions most approximate to the actual local perceptual optima that are mutually "same" or "inconclusive".

\section{- Step 2: Statistical Flicker Estimation}

Among the local perceptual optima obtained above, we estimate their flickers under potential Internet changes. We conduct the statistical estimation based on previous long-term records of Internet statistics of the same connection.

We describe the network condition by three parameters: the Internet delay average $\bar{\phi}$, the jitter $j$ and the bandwidth availability $r$. The Internet random losses are negligible, and we assume they can be concealed by the media codec. Except $\bar{d}_{0}$, whose variation is negligible, the other two parameters can be described as random variables $J$ and $R$. We assume their mutual independence. Their distributions can be estimated based on long-term record statistics. We also describe the size of a time-correlated multiview video frame $s v$ as a known distribution $S V$, and the codec latency for each time-correlated multiview video frame is a constant $d_{c}$.

Given a fixed bandwidth $r$, the video macroframe delay is represented as a function $r$ :

$$
D_{V}(r)=S V / r+\bar{d}_{0}+J+d_{c}
$$

As discussed in Section 2.A, the video delay includes the codec delay $d_{c}$, the Internet delay $\left(\bar{d}_{0}\right.$ and $\left.J\right)$, and the transmission delay $S V / r . D_{V}(r)$ is decided by the distributions of both $J$ and $S V$. On the other hand, the audio frame delay is mainly decided by the Internet delay $\bar{\phi} b$ and $J$. The audio codec and transmission delays are negligible.

$$
D_{A}=\bar{d}_{0}+J
$$

Suppose the candidate local perceptual optimum is $\vec{e}=\left\{e_{V}^{c}, e_{A}^{c}, e_{D}^{c}, e_{S}^{c}\right\}$ with the corresponding control values $\operatorname{EED}_{V}^{c}$ and $\operatorname{EED}_{A}^{c}$. We compute the percentage of video macroframes or audio frames (denoted as $h_{V}$ and $h_{A}$ ) that arrive within $\operatorname{EED}_{V}^{c}$ and $\operatorname{EED}_{A}^{c}$, given the Internet jitter and transmission delay variations based on $S$ and $J$ distributions.

$$
h_{A}=P\left(D_{A} \leq \operatorname{EED}_{A}^{c}\right), h_{V}(r)=P\left(D_{V}(r) \leq \operatorname{EED}_{V}^{c}\right)
$$


Here, $P(\cdot)$ represents the probability of the input expression. Because $\left(1-h_{A}\right)$ and $\left(1-h_{V}\right)$ are actually the unavailable percentage of audio frames and video macroframes, which directly decide $e_{A}$ and $e_{V}$ (discussed in Section 2.A), we know the mapping $F_{V} / F_{A}$ from $h_{V} / h_{A}$ to $e_{V} / e_{A}$ :

$$
F_{V}: h_{V} \mapsto e_{V} \quad F_{A}: h_{A} \mapsto e_{A}
$$

Therefore, $\left|F_{A}\left(h_{A}\right)-e_{A}^{c}\right|$ and $\left|F_{V}\left(h_{V}\right)-e_{V}^{c}\right|$ can be used to approximate the change of the audio and video signal quality from $\vec{e}_{A}^{c}$ and $\vec{e}_{V}^{c}$ due to the Internet condition changes.

As the bandwidth $r$ varies according its distribution $R$, the expected difference of the media signal quality (denoted as $\delta e_{V}$ and $\delta e_{A}$ ) over $R$ at $e^{c}$ becomes:

$$
\begin{gathered}
\delta e_{A}\left|e_{A}^{c}=\right| F_{A}\left(h_{A}\right)-e_{A}^{c} \mid \\
\left.\delta e_{V}\right|_{V} ^{c}=\int_{r}\left|F_{V}\left(h_{V}\right)-e_{V}^{c}\right| f_{R}(r) d r
\end{gathered}
$$

By plugging Eqn. 5.16 and 5.17 into Eqn. 5.6, we finally come up with the expected flicker $f d a$ at the candidate $\vec{e}^{c}$, assuming $\delta e_{A}$ and $\delta e_{V}$ are mutually independent.

$$
\left.f d a\right|_{\vec{e}^{c}}=\left.w_{A} \cdot \delta e_{A}\right|_{e_{A}^{c}}+\left.w_{V} \cdot \delta e_{V}\right|_{V} ^{c}
$$

\section{- Step 3: Online Adaptation}

The receiver MPS updates the operating point periodically based on the results from Step 1 and 2. It also takes into account the system adaptation flickers.

We let the set of local perceptual optima be $\overrightarrow{\mathcal{E}}^{C}=\left\{\vec{e}^{c}\right\}$, and their corresponding statistical flicker estimations be $\left.f d a\right|_{\vec{e}^{c}}$ (Eqn. 5.18). We assume the operating point before the MPS update be $\vec{e}^{t}$. We compute the flicker between $\vec{e}^{-1}$ and $\vec{e}^{c}$ incurred as the result of the system adaptation, and denote it as $\left.f d b\right|_{\vec{e}^{c}}=f d\left(\vec{e}^{c}, \vec{e}^{1}\right)$ (Eqn. 5.6).

The flickers are introduced jointly by both Internet variations and system adaptations. We use a heuristic weighted linear function: $w_{1} \cdot f d a+w_{2} \cdot f d b$ to estimate their combined impact. The reason we study $f d a$ and $f d b$ separately is that various multimedia applications usually attach different importance to the two factors, so we are able to assign them different weights in each application. Hence the receiver selects the best operating point among $\overrightarrow{\mathcal{E}}^{C}$ that can minimize the combined flicker impact.

$$
\vec{e}^{-o p t}=\arg \min _{\vec{e}^{c} \in \overrightarrow{\mathcal{E}}^{C}}\left\{\left.w_{1} \cdot f d a\right|_{\vec{e}^{c}}+\left.w_{2} \cdot f d b\right|_{\vec{e}^{c}}\right\}
$$



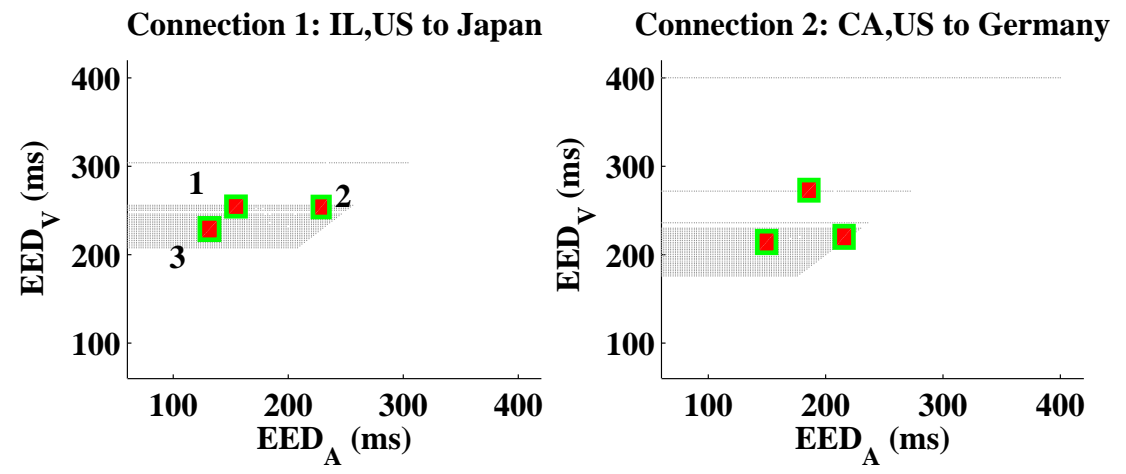

Figure 5.12: Grey dots: all non-dominated points on an operating plane. Red boxes: local perceptual optima returned by our perception-based genetic algorithm.

By computing $\mathrm{EED}_{V}$ and $\mathrm{EED}_{A}$ values from $e^{\text {opt }}$, the receiver then sets its buffer sizes and schedules the media packets to be sent to the output devices accordingly.

\subsubsection{Evaluation Results}

\section{- Descriptions of Evaluation Configurations}

To evaluate our perception-driven MPS scheme, we employ the same TEEVE tested as in Section 4. One audio stream and up to 3 multiview video streams are prerecorded and used at each site. The video codec delay in our testbed is around $d_{c}=70 \mathrm{~ms}$. We use UDP for the media distribution. The Internet emulator developed in Section 4 is also deployed between the sender and receiver gateways to replay the PlanetLab UDP delays and losses. To further measure the bandwidth impact, we use $t c$ software to control the upper bound of the data rate at the sender gateway. The generalized preference results from our user study (Section 4.5) are employed as an input in our MPS adaptations. Here, we only present the teleimmersive conferencing application where two parties are conducting a social conversation (CONV).

\section{- Local Perceptual Optima}

Fig. 5.12 shows an example of local perceptual optima solutions (red boxes) returned from the perceptionbased genetic algorithm under two network conditions. We only show them in the two-dimensional control space, because the corresponding four-dimensional objective quality points are hard to visualize. As a comparison, the figure also shows all non-dominated solutions in the current operating plane. Connection 1 has an average delay of $120 \mathrm{~ms}$ and a bandwidth of $15 \mathrm{Mbps}$, while connection 2 has a delay average of $90 \mathrm{~ms}$, and a bandwidth of 20 Mbps. The average jitter in both connections is around $15 \mathrm{~ms}$. 
Our genetic approach is run for 100 iterations for each connection. The results show the diversity of the perceptual elitists. The values of $\mathrm{EED}_{V}$ and $\mathrm{EED}_{A}$ imply their objective qualities. For example, in connection 1, solution 1 outputs a better interactive quality than solution 2 , but a worse synchronization quality and audio signal intelligibility. On the other hand, solution 1 represents a worse interactive quality, but a better video signal intelligibility than solution 3 .

\section{- Evaluation of Perception-driven MPS}

We evaluate our perception-driven MPS under multiple Plantlab traces with diverse delay, jitter and loss characteristics. As a comparison, we also evaluate the non-perception based approach discussed in Section 4, where we set $\mathrm{EED}_{V}=\mathrm{EED}_{A}+80$ (multiple video streams are shared with the same $\mathrm{EED}_{V}$, as prescribed in Section 5.1). Here, $80 \mathrm{~ms}$ is the maximum audio-visual skew that cannot be noticed [20].

Fig. 5.13 and Fig. 5.14 present the comparison results of the two algorithms between IL,USA and the Netherlands with a fixed bandwidth of 15 Mbps over 100-second duration. In both algorithms, $D_{V}$ and $D_{A}$ can be computed using Eqn. 5.12 and 5.13. For the perception-driven MPS, we set the normalized weights in the flicker formulations (Eqn. 5.6) to be: $w_{V}=1 / 20, w_{A}=1 / 4, w_{D}=1 / 1600$ and $w_{S}=1 / 400$. We also heuristically set $w_{1}=0.2$ and $w_{2}=0.8$ in Eqn. 5.19. For the non-perception based algorithm, we set $\mathrm{UR}_{\mathrm{TH}}\left({ }^{\prime} \mathrm{V}\right.$ ') $=\mathrm{UR}_{\mathrm{TH}}\left({ }^{\prime} \mathrm{A}\right.$ ') $=0.05$. In both algorithms, we update the MPS control every 10 seconds. The most recent 10 -second duration is used for characterizing the short-term network conditions by both algorithms.

Fig. 5.13(a) and (b) present the variations of $\mathrm{EED}_{V}$ and $\mathrm{EED}_{A}$ in response to Internet dynamics. They show that both algorithms are able to adapt the video and audio receiver buffer sizes based on the Internet jitter. However, the perception-driven MPS outputs far smaller fluctuation magnitudes. This can be explained by two reasons. First, a local optimal objective quality point may not have the best media signal quality, so both $\mathrm{EED}_{V}$ and $\mathrm{EED}_{A}$ do not have to accommodate all delay spikes. Second, the perception-driven MPS algorithm takes into account the flicker effects caused by the system control, so it purposefully reduces EED and $\mathrm{EED}_{A}$ fluctuations. In addition, we find that the robustness of the non-perception based algorithm to a sudden Internet change depends on the timing window size of the short-term Internet traffic statistics, while the perception-driven MPS sets a more reasonable value for both $\mathrm{EED}_{V}$ and $\mathrm{EED}_{A}$, because of the internal statistical flicker estimation mechanism.

Within each 10-second duration, we calculate the operating points of both algorithms (Fig. 5.14) and the resulting flickers experienced by users under the combined impacts of both receiver control updates the real Internet dynamics (Fig. 5.13(c)). We show that our perception-driven MPS scheme outputs a smaller flicker 

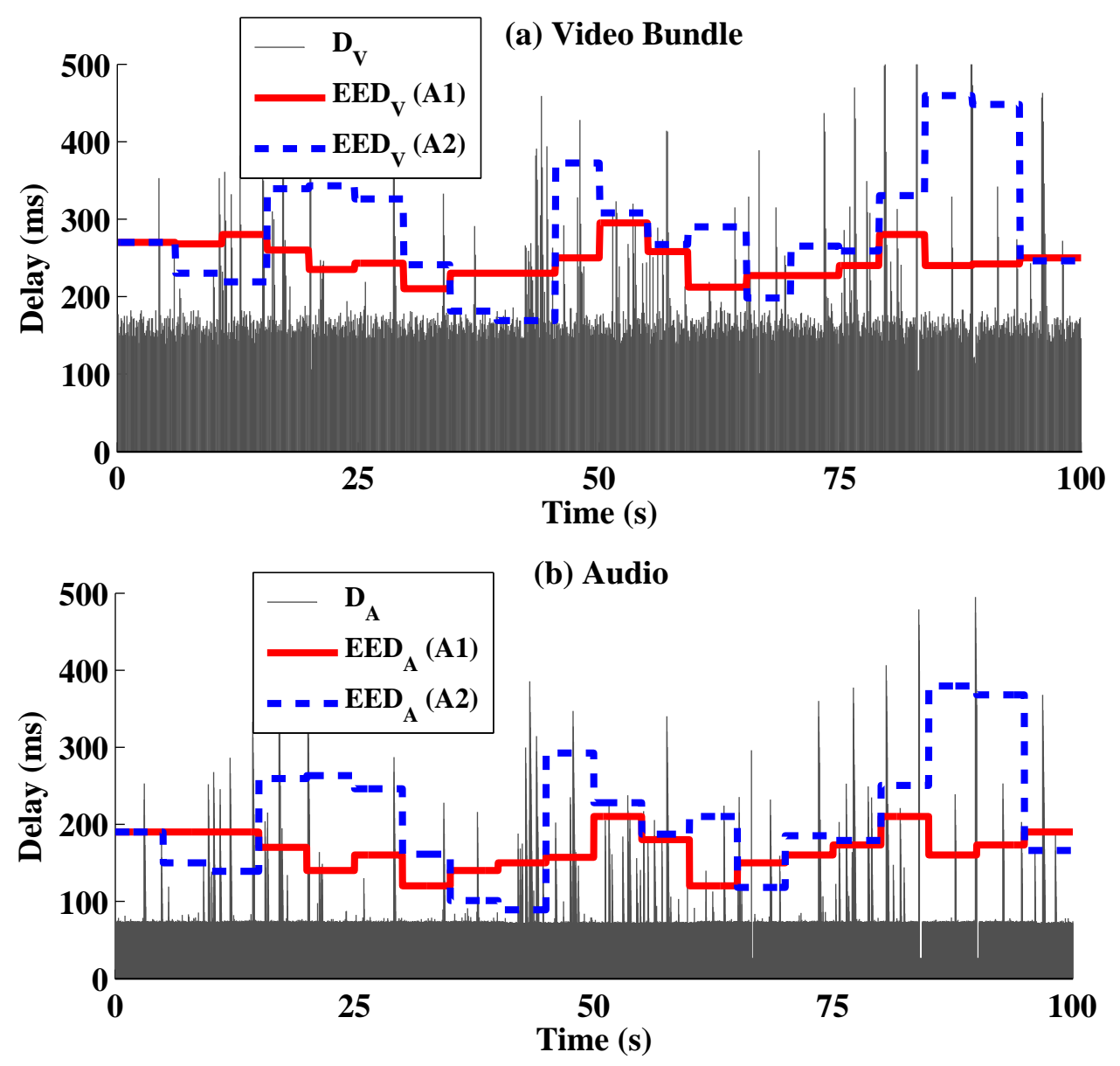

(c) Actual Flicker

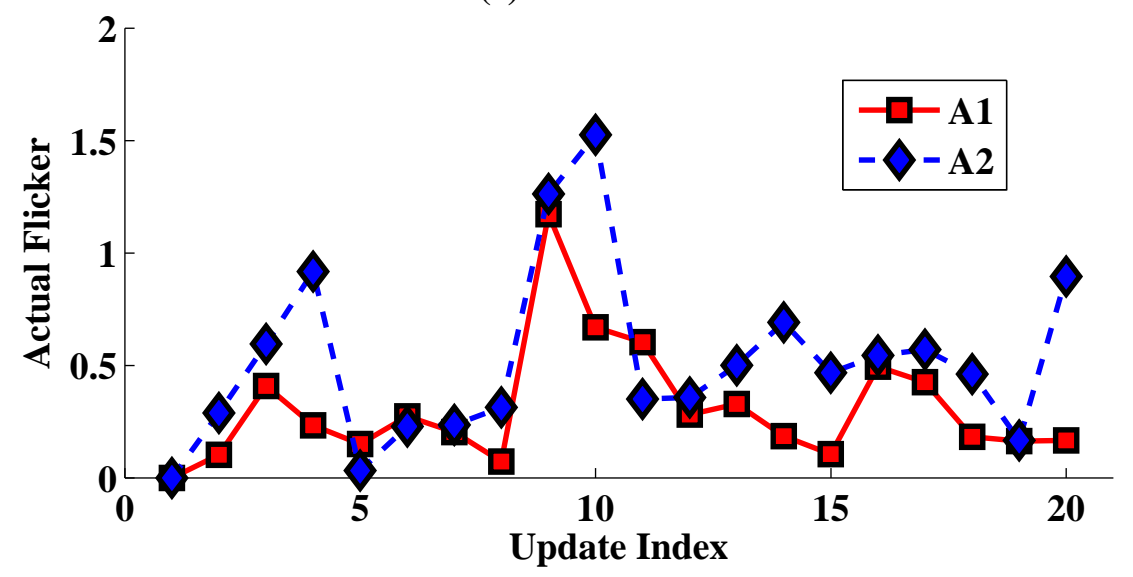

Figure 5.13: Comparison between perception-driven MPS (A1) and non-perception based algorithm (A2) [1]. Internet traces are between IL,USA to the Netherlands. Bandwidth is 15 Mbps. $D_{V}$ and $D_{A}$ are defined in Eqn. 5.12 and 5.13. Flicker values are shown for each receiver update. 
(a) $\mathbf{x}_{\mathbf{V}}$

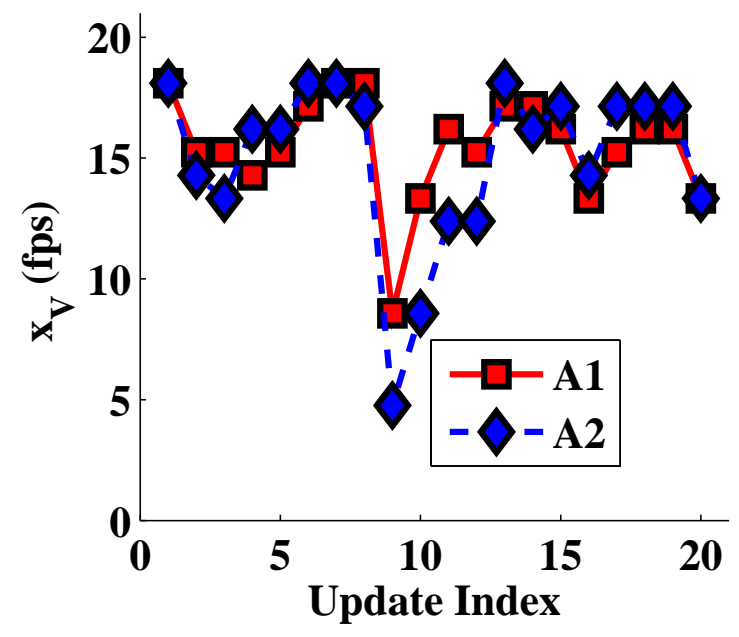

(c) $\mathbf{x}_{\mathbf{D}}$

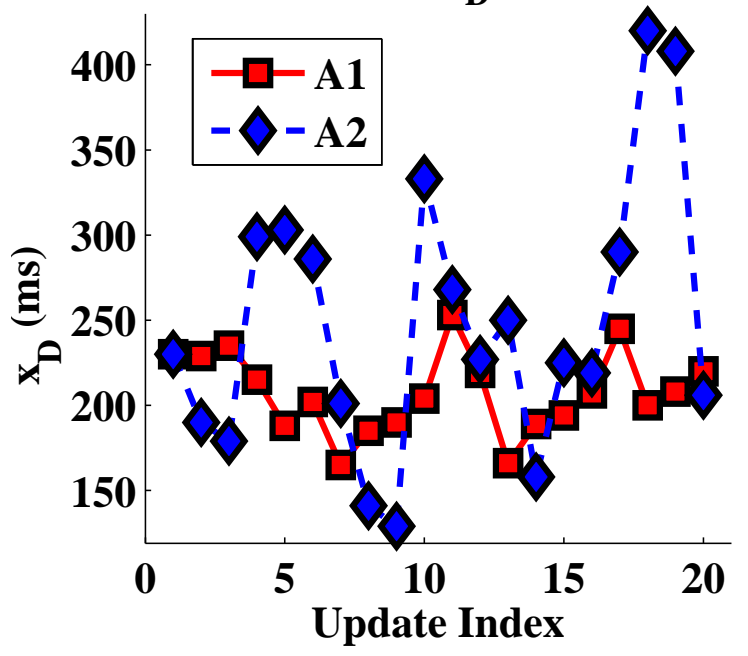

(b) $\mathbf{x}_{\mathbf{A}}$

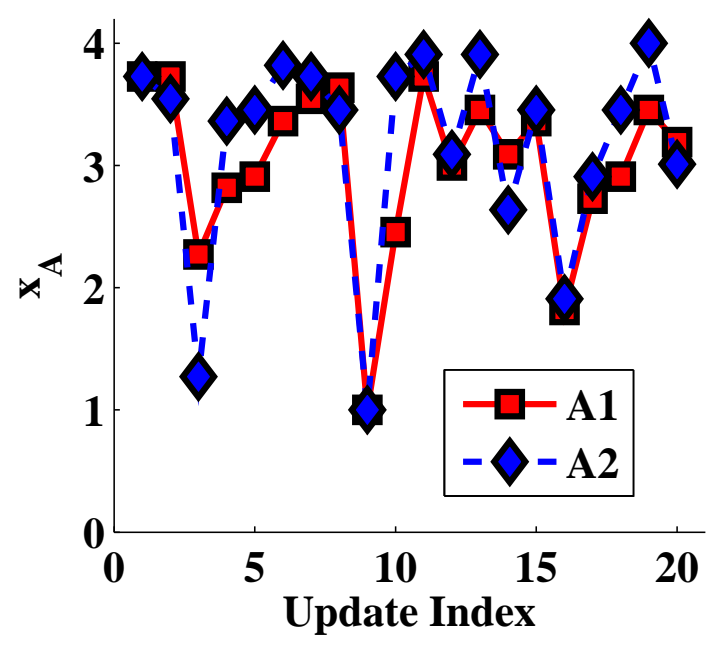

(d) $\mathbf{x}_{\mathrm{S}}$

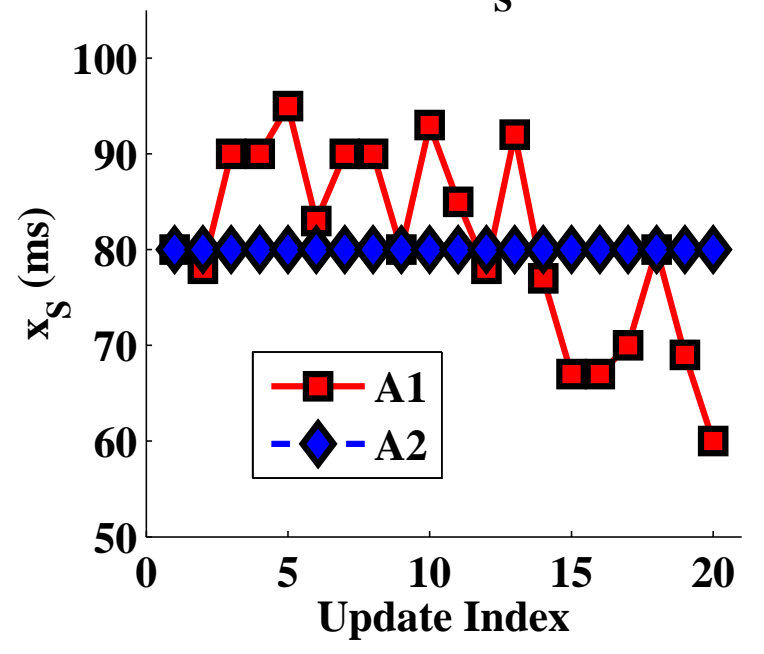

Figure 5.14: Objective quality metric values for each 5-sec duration. A1: perception-driven MPS. A2: non-perception based algorithm. 
compared to the non-perception based version, and that it offers less fluctuations in the audio and video signal intelligibility, and the interactive quality. These results prove the credit of our perception-driven MPS algorithm.

\subsection{Summary}

In this chapter, we extract objective metrics capturing the streaming media quality and the flicker impact in two teleimmersive applications. We present a systematic methodology to evaluate the effectiveness and limitation of CMOS. To address the issue of CMOS inconclusiveness, we propose the subjective preference metric for describing the diversity of user votes. By employing the generalized subjective evaluation results using new preference metric, we discuss the design of the perception-driven online media presentation scheduling approach for the multimedia synchronization for best subjective user satisfactions.

\subsection{Table of Abbreviations and Notations}

\section{List of Abbreviations in Chapter 5:}

$\begin{array}{ll}\text { TEEVE } & \text { Teleimmersive Environment for EVErybody } \\ \text { ITU } & \text { International Telecommunication Union } \\ \text { MOS } & \text { Mean-Opinion-Score } \\ \text { DMOS } & \text { Degradation MOS } \\ \text { CMOS } & \text { Comparative MOS } \\ \text { ACR } & \text { Absolute Category Rating } \\ \text { CCR } & \text { Comparative Category Rating } \\ \text { DCR } & \text { Degradation Category Rating } \\ \text { PESQ } & \text { Perceptual Evaluation of Speech Quality } \\ \text { QoS } & \text { Quality of Service } \\ \text { QoE } & \text { Quality of Experience } \\ \text { CI } & \text { Confidence Interval } \\ \text { CIF } & \text { Common Intermediate Format }\end{array}$




$\begin{array}{ll}\text { QCIF } & \text { Quarter Common Intermediate Format } \\ \text { SVM } & \text { Support Vector Machine } \\ \text { W.L.O.G } & \text { Without Loss of Generality } \\ \text { TCP } & \text { Transmission Control Protocol } \\ \text { UDP } & \text { User Datagram Protocol } \\ \text { IP } & \text { Internet Protocol } \\ \text { VoIP } & \text { Voice over Inter Protocol } \\ \text { EED } & \text { End-to-End Delay } \\ \text { HRD } & \text { Human Response Delay } \\ \text { CONV } & \text { Conversational Activity } \\ \text { COLL } & \text { Collaboration Activity } \\ \text { LAN } & \text { Local Area Network } \\ \text { MPS } & \text { Media Presentation Scheduling }\end{array}$

\section{List of Notations in Chapter 5:}

$\begin{array}{ll}e_{A} & \text { Dimension of audio signal intelligibility } \\ e_{V} & \text { Dimension of video signal intelligibility } \\ e_{S} & \text { Dimension of synchronization quality } \\ e_{D} & \text { Dimension of interactive quality } \\ \vec{e} & \text { Streaming media quality point: } \vec{e}=\left\{e_{V}, e_{A}, e_{D}, e_{S}\right\} \\ \mathrm{EED}_{V} & \text { Video one-way end-to-end delay } \\ \mathrm{EED}_{A} & \text { Audio one-way end-to-end delay } \\ \overline{\mathrm{EED}} & \text { Average one-way end-to-end delay: } \overline{\mathrm{EED}}=\left(\mathrm{EED}_{A}+\mathrm{EED}_{V}\right) / 2 \\ f d & \text { Flicker distance } \\ w_{A} & \text { Coefficient of audio signal intelligibility for } f d \\ w_{V} & \text { Coefficient of video signal intelligibility for } f d \\ w_{S} & \text { Coefficient of synchronization quality for } f d \\ w_{D} & \text { Coefficient of interactive quality for } f d\end{array}$




\begin{tabular}{|c|c|}
\hline$Q S$ & Subjective quality \\
\hline$Q$ & Maximum-possible impairment of $e_{V}$ \\
\hline$c$ & Slope of exponential curve \\
\hline$\vec{g}$ & Input control vector: $\vec{g}=\left\{\mathrm{EED}_{V}, \mathrm{EED}_{A}\right\}$ \\
\hline$\vec{g}^{1}$ & Non-dominated solution \\
\hline$\vec{g}^{2}$ & Perceptual Elitist \\
\hline$\delta g$ & Minimal Euclidean distance of $g$ \\
\hline $\bar{d}_{0}$ & Internet delay average \\
\hline$d_{c}$ & Codec latency for time-correlated multiview video frame \\
\hline$s v$ & Size of time-correlated multiview video frame: random variable \\
\hline$S V$ & Size of time-correlated multiview video frame: distribution \\
\hline$j$ & Internet jitter: random variable \\
\hline$J$ & Internet jitter: distribution \\
\hline$r$ & Bandwidth availability: random variable \\
\hline$R$ & Bandwidth availability: distribution \\
\hline$D_{A}$ & Audio delay \\
\hline$D_{V}$ & Video delay \\
\hline$P$ & Probability \\
\hline$h_{A}$ & Percentage of audio frames arrive within $\mathrm{EED}_{A}$ \\
\hline$h_{V}$ & Percentage of time-correlated multiview video frames arrive within $\mathrm{EED}_{V}$ \\
\hline$F_{A}$ & Mapping: $F_{A}: h_{A} \mapsto e_{A}$ \\
\hline$F_{V}$ & Mapping: $F_{V}: h_{V} \mapsto e_{V}$ \\
\hline$\delta e_{A}$ & Expected difference of $e_{A}$ \\
\hline$\delta e_{V}$ & Expected difference of $e_{V}$ \\
\hline$f d a$ & Flicker due to Internet quality changes \\
\hline$f d b$ & Flicker due to online system adaption \\
\hline
\end{tabular}




\section{CHAPTER 6}

\section{SYNCHRONIZATION IMPLEMENTATION IN TELEIMMERSION}

In this chapter, we will discuss the synchronization implementations in the real multi-site teleimmersive system. We will build different synchronization service components at both sender and receiver sites, that have been discussed so far in this dissertation. As of now, we have only realized the deployment of non-perception based algorithms, whose implementation details and performance evaluations are the main focuses of this chapter.

\subsection{An Introduction of Real Teleimmersive System Implementation}

We first provide a brief introduction of existing implementation architecture of real teleimmersive systems in our Illinois TEEVE testbed (Fig. 6.1). Each site includes the following must-have components:

1. Sensory inputs. Multiple multimedia input sensors (e.g., 3D cameras and microphones) are equipped at each site. Each multimedia sensor is attached to one specialized computation machine for real-time encoding and processing, via USB, IEEE 1394 or wireless connections.

2. Producer wrapper. A producer wrapper service daemon is located between the computation machine and the gateway, for adding the real-time protocol (RTP) header to each media frame. We have expanded the standardized RTP header to describe the time-correlated multi-modal multi-stream characteristics in the teleimmersive setting. Fields in the header include the identifier of the sensory streams, the sequence number of the media frames, and the type of each media modality. A detailed description of the RTP header will be presented in Section 6.2.

3. Gateway. The gateway aggregates the local multi-modal media data, and sends the whole multistream bundle to the remote sites. Due to the firewall issue, only the full-mesh topology is realized in the existing implementation. The gateway also sends both local and remote media data to the local consumer wrapper service daemon. 


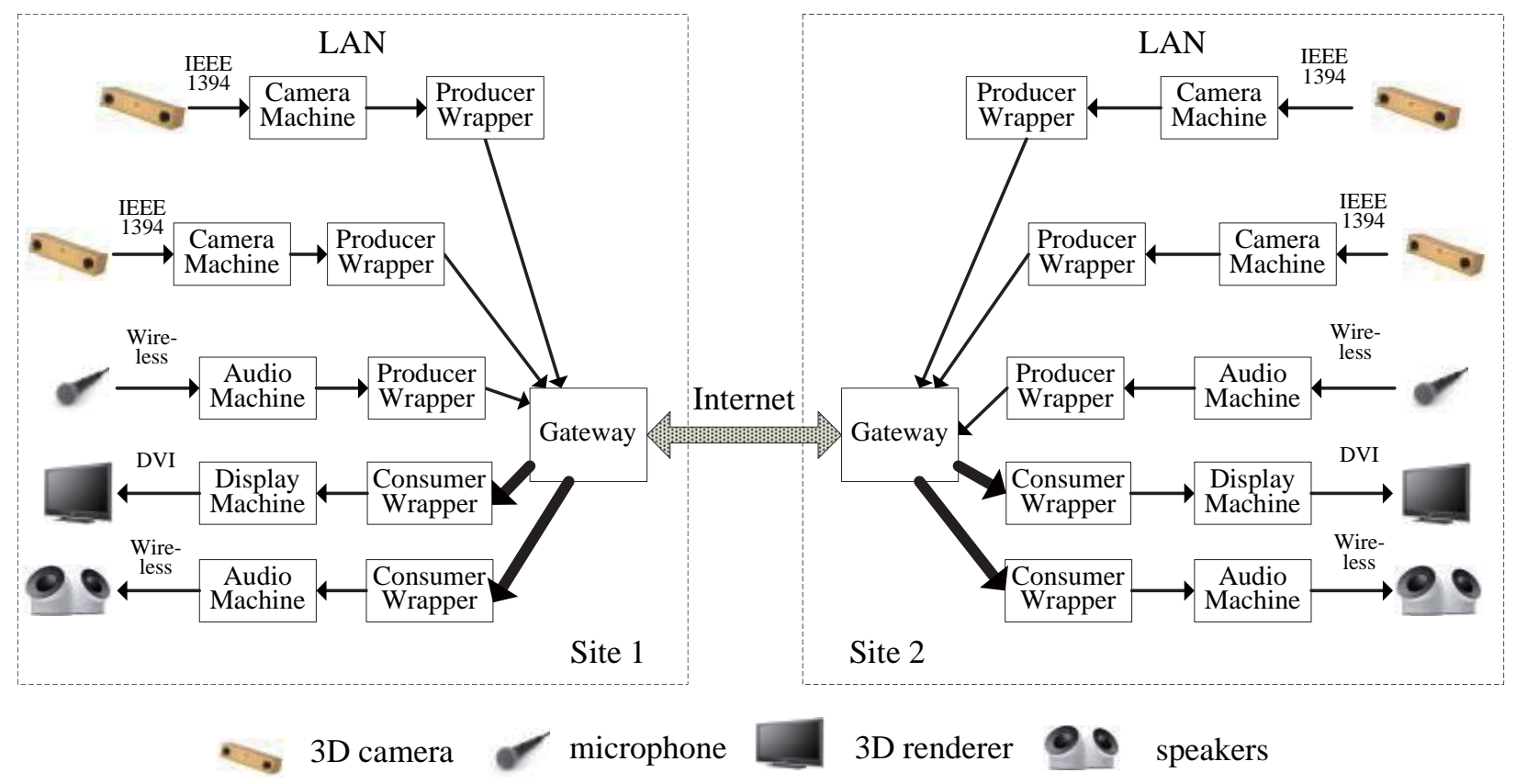

Figure 6.1: Configuration and implementation of real teleimmersive systems.

4. Consumer wrapper. The consumer wrapper is responsible for removing the RTP header, and sending the original encoded media frames to the video and audio decoding machines.

5. Media outputs. Each media frame is decoded and presented at the corresponding output devices, like the video display and audio speaker.

Note that due to the heterogeneity and mobility of next-generation multimedia sensors, a computation (encoding and decoding) machine may not be needed. For example, in teleimmersion, a specialized wireless body sensor can directly process the media frames and send them to the corresponding producer wrapper.

\subsection{Implementation of Synchronization Service Components}

\subsubsection{Implementation Overview}

Fig. 6.2 shows an overview of the synchronization service components that we have implemented in the real teleimmersive systems. In order to access the global timestamps, the system clocks must be physically or virtually synchronized both across and within the distributed sites. The RTP header needs to be expanded to reflect the dependencies among the multi-modal multi-stream data. We have implemented the synchronization point identification algorithm at the senders, and media presentation scheduling algorithm 


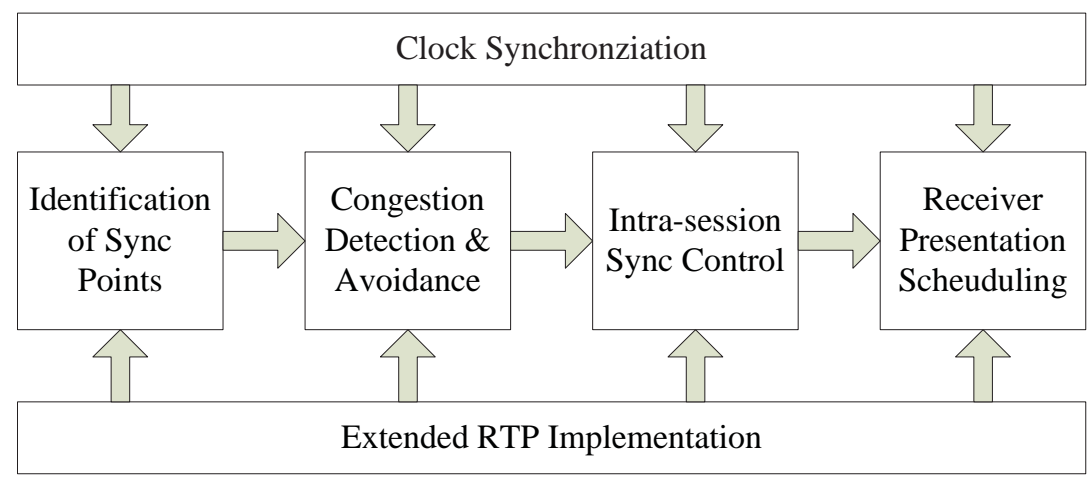

Figure 6.2: Implementation overview of synchronization service components in teleimmersion.

at the receivers. We have also realized the congestion detection and avoidance mechanism, and centralized intra-session synchronization control protocols during the implementation.

\subsubsection{RTP Format}

Table. 6.1 shows the expanded RTP header for the multi-modal multi-stream synchronization in teleimmersion. We rely on the Google ProtoBuf ${ }^{1}$ to facilitate the implementation.

The table lists the functionality, type, number of bytes, name and explanation of each field in the RTP header. It also specifies whether this field is a must. Note that the functionality can be classified into two categories: (1) general, representing the fields that have existed in the previous teleimmersive implementation without the synchronization functionality, and (2) sync, indicating the fields added for synchronization purposes. The contributions of this dissertation lie at the second category.

\subsubsection{Clock Synchronization}

Clock synchronization in teleimmersion maintains two-step procedure to guarantee high-precision global timestamps, as described in Fig. 6.3.

1. Physical clock synchronization. At the start of a multi-site teleimmersive session, all producer and consumer wrappers as well as gateways within the session are physically synchronized using NTP. This allows minimal clock skews across distributed sites. However, clock drifts can be incurred online, NTP operation at the presence of bandwidth-savvy multimedia traffic is costly. In our implementation, we have to rely on the virtual clock synchronization to remedy the clock drifts, whose details are discussed below.

\footnotetext{
${ }^{1}$ Google Protocol Buffers: http: //code.google.com/p/protobuf /
} 
Table 6.1: Expanded RTP header in teleimmersion

\begin{tabular}{|c|c|c|c|c|c|}
\hline Functionality & Required? & Type & \#Bytes & Field Name & Explanation \\
\hline \multirow{5}{*}{ General } & optional & uint32 & 4 & version & Version of the RTP header \\
\hline & required & uint64 & 8 & stream_uid & $\begin{array}{l}\text { Stream_uid are different for all } \\
\text { sensory streams within the ses- } \\
\text { sion. The field contains the } \\
\text { site information where a sensory } \\
\text { stream is sourced. }\end{array}$ \\
\hline & required & uint32 & 4 & payload_type & $\begin{array}{l}\text { Payload type indicates the media } \\
\text { modality of the sensory stream: } \\
\text { video, audio, haptics, and etc ... }\end{array}$ \\
\hline & required & uint32 & 4 & payload_subtype & $\begin{array}{l}\text { Payload subtype represents sub- } \\
\text { category of each media modal- } \\
\text { ity. For example, for video } \\
\text { modality, payload_subtype can } \\
\text { be 2D_video and 3D_video }\end{array}$ \\
\hline & required & uint32 & 4 & frame_number & $\begin{array}{l}\text { Frame index of each media frame } \\
\text { within a sensory stream }\end{array}$ \\
\hline \multirow[t]{3}{*}{ Sync Added } & required & uint32 & 4 & tos & $\begin{array}{l}\text { Specify whether the sensory } \\
\text { stream represents an intra-media } \\
\text { or intra-bundle synchronization } \\
\text { reference (inputted via user inter- } \\
\text { face) }\end{array}$ \\
\hline & required & uint64 & 8 & timestamp & $\begin{array}{l}\text { Timestamp that media frame is } \\
\text { captured }\end{array}$ \\
\hline & required & uint64 & 8 & tob_offset & $\begin{array}{l}\text { Buffer size offset for intra- } \\
\text { session synchronization }\end{array}$ \\
\hline General & required & bytes & any & payload & $\begin{array}{l}\text { Media frame content: variable } \\
\text { bytes }\end{array}$ \\
\hline
\end{tabular}




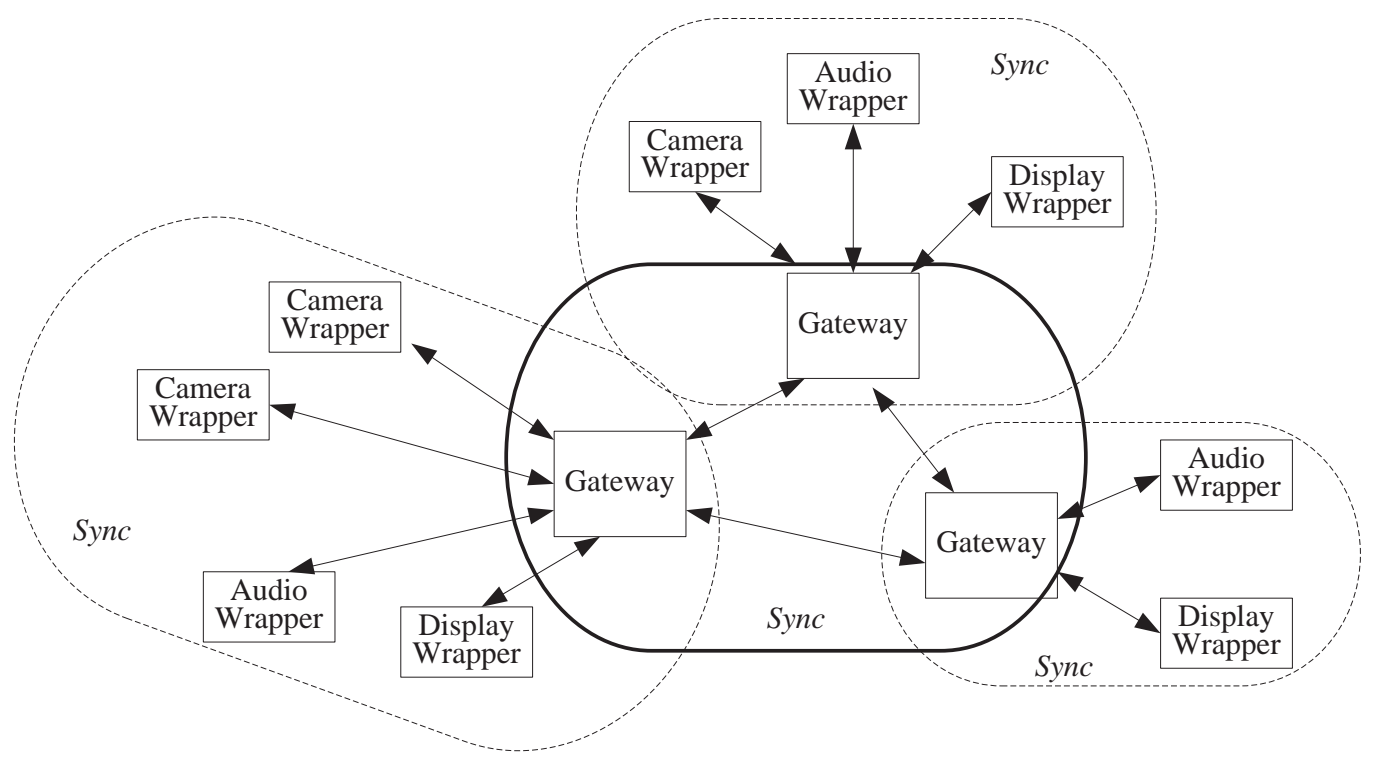

Figure 6.3: Hierarchical virtual clock synchronization in teleimmersion.

2. Virtual clock synchronization. During the system run time, we follow a hierarchical fashion to achieve the virtual clock synchronization (i.e., estimating the clock skews across different machines without changing their physical clock time). First, we synchronize distributed gateways at each site, by sending/receiving a sequence of periodic round-trip synchronization control packets to/from each other, and estimating the resulting clock skews using Eqn. 2.1. The one-way delay from one gateway to the other can also be computed by halving the round-trip latency. Second, at each site, we synchronize multimedia computation machines, by sending/receiving round-trip control packets to/from the local gateway, and estimating the resulting clock skews. In both cases, we send the round-trip packets every $100 \mathrm{~ms}$.

\subsubsection{Identification of Synchronization Points}

We implement two components to identify the synchronization points (Fig. 6.4), based on the algorithm that we have proposed in Section 3.3. We decide to realize the identification at the producer wrappers, because the wrapper machine is the earliest controllable component that can be physically synchronized.

First, at each clock-synchronized producer wrapper, we estimate the captured timestamp of each received media frame, and specify the timing information in the timestamp field of the RTP header. Assuming the arrival time of the $k$-th media frame of a sensory stream at the corresponding wrapper is $t_{a r}(k)$, its actual captured timestamp at the sensory input $t_{e}(k)$ can be estimated by: 


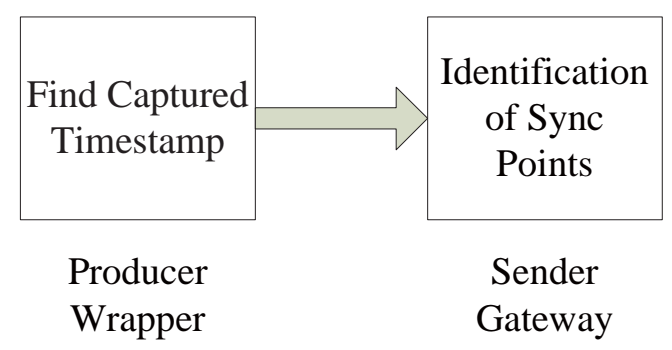

Figure 6.4: Identifying the synchronization points.

$$
t_{e}(k)=t_{a r}(k)-T_{\text {cap }}(k)
$$

where $T_{\text {cap }}(k)$ is the encoding overhead. There are two options in the existing implementation to access $T_{\text {cap }}(k)$. (1) Static: we use a static encoding time inputted via the user interface. This option is often employed for the types of media modalities demanding constant or almost constant computation overhead, e.g., audio and haptics. (2) Dynamic. We infer the encoding time from the inter-arrival period of two consecutive media frames $k-1$ and $k$ :

$$
T_{\text {cap }}(k)=t_{a r}(k)-t_{a r}(k-1)
$$

This option is used for computation-intensive media modality whose encoding overhead depends on the amounts of information carried in each media frame, e.g., 2D and 3D video.

Second, based on the RTP timestamp information, we then identify the synchronization points across the multi-modal multi-stream data at each gateway.

\subsubsection{Congestion Detection and Avoidance}

When multi-modal media data are being streamed from one site to the other, congestions can happen at an insufficient bandwidth. This will increase the packet drop rate when UDP is employed, or reduce the data sending rate when TCP is used. Hence, in our teleimmersive systems, we actively detect the congestion status at each gateway, by monitoring the sending rate of the TCP traffic, or the drop rate of the UDP traffic. We place a heuristic tolerable threshold for both metrics. In the existing implementation, if the TCP sending rate goes below $5 \mathrm{Mbps}$, or UDP drop rate beyond 5\%, we specify that a congestion happens. In both cases, we simply drop all video data currently waiting in the outbound queue (to remote sites), in order to avoid further congestions. We assume that the bandwidth demands for other media modalities are negligible. 


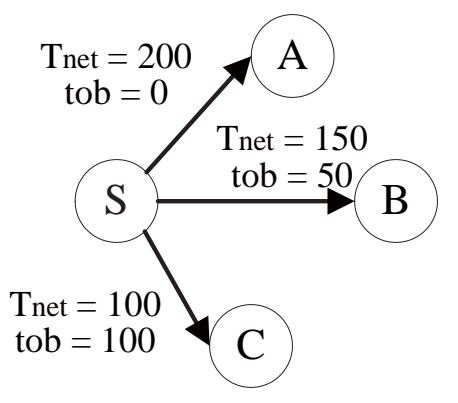

Inter-receiver Sync

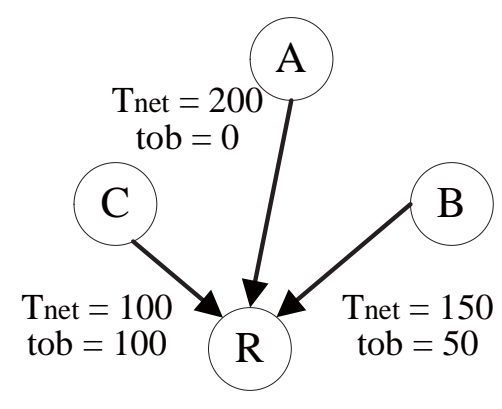

Inter-sender Sync

Figure 6.5: Intra-session synchronization in teleimmersion. Unit: ms.

\subsubsection{Intra-session Synchronization Mechanism}

We support both inter-sender and inter-receiver synchronization. However, they cannot operate at the same time in the existing synchronization implementation. We must select only one synchronization mechanism during the system run time. To make our teleimmersive system universal to all applications, we elect the intra-session synchronization reference (i.e., reference site) to be the one that experiences the longest (average) one-way network latency $T_{n e t}$ during the media distribution. $T_{n e t}$ can accessed by sending roundtrip control packets, as discussed in Section 6.2.3. Fig. 6.5 shows an example of the inter-sender and inter-receiver synchronization, where different sender/receiver sites experience heterogeneous $T_{n e t}$. In both cases, site $\mathrm{A}$ is always elected as the reference due to its longest $T_{n e t}$.

By letting $T_{n e t}^{\max }$ to be the maximal $T_{n e t}$ within the session, we compute for each site the tob value, which is the difference between its $T_{n e t}$ and the maximum $T_{n e t}^{\max }:$ tob $=T_{\text {net }}^{\max }-T_{n e t}$. The tob value represents the offset (i.e., the additional size) of the receiver buffer at each site in order to realize the intrasession synchronization. It is specified in the RTP header, either by the sender gateway for the inter-receiver synchronization, or by the receiver gateway for the inter-sender synchronization.

\subsubsection{Receiver Presentation Scheduling}

To avoid the complications behind the distributed control of scalable output devices at the receiver sites, we perform a centralized presentation scheduling at each receiver gateway, meaning that we insert the buffering latencies, for all media frames, at the gateways instead of decoding and presentation machines, in order to compensate for synchronization skews.

For multi-modal multi-stream synchronization within each bundle, we rely on the TBuffer algorithm discussed in Section 4.4 to decide the presentation scheduling, by consulting the synchronization reference 
information (tos field) specified in the RTP header. We suppose $T_{h}$, the buffer hold time of each media frame at the receiver gateway, is the output of TBuffer algorithm. To further realize the intra-session synchronization across multiple bundles, we slightly modify TBuffer, and prescribe that the actual buffering latency of the media frame be $T_{h}+$ tob. We do not impose strict intra-session synchronization constraint mainly due to the implementation flexibility.

Note that we only update the buffer statuses periodically (say, every 10-60 ms).

\subsection{Performance Validation}

\subsubsection{Test Scenarios}

We evaluate the performance of synchronization service components in the real teleimmersive systems. Same as Section 4.4, we use $t c$ software to introduce delays and jitter, and throttle the bandwidth availability. Fig. 6.6 shows three scenarios we would like to test:

- Case 1: two-site system (Fig. 6.6(a)), where the sender site outputs two video streams and one audio stream. Our focus is to evaluate the performance of intra-stream, intra-media and intra-bundle synchronization. We introduce delays and jitter for both video and audio traffics.

- Case 2: three-site system with one active sender site (Fig. 6.6(b)), where the single sender outputs two video streams and one audio stream. We aim to evaluate the inter-receiver synchronization between the two receivers. We impose delays and jitter on both video and audio traffics to one of the receiver sites, but maintain minimal latency to the other receiver.

- Case 3: three-site system with two active sender sites (Fig. 6.6(c)), where each sender outputs one video stream and one audio stream. We target at validating the performance of the inter-sender synchronization at the third receiver site. We exert delays and jitter on both video and audio traffics from one of the sender sites, but maintain minimal latency from the other sender.

In all the three scenarios, we set the outbound bandwidth availability at each sender site to be $50 \mathrm{Mbps}$.

\subsubsection{Evaluation Results}

Fig. 6.7, 6.8 and 6.9 represent the media synchronization results for the above three cases. As discussed in the TBuffer algorithm (Eqn. 4.5 and 4.6), we plot, for each media frame, its $D_{r g}$ (i.e., the latency that 
Fig (a)

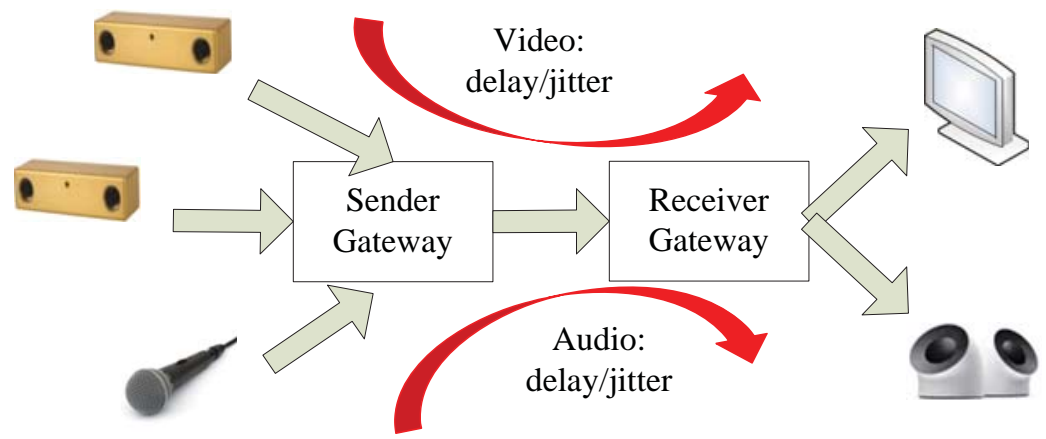

Fig (b)

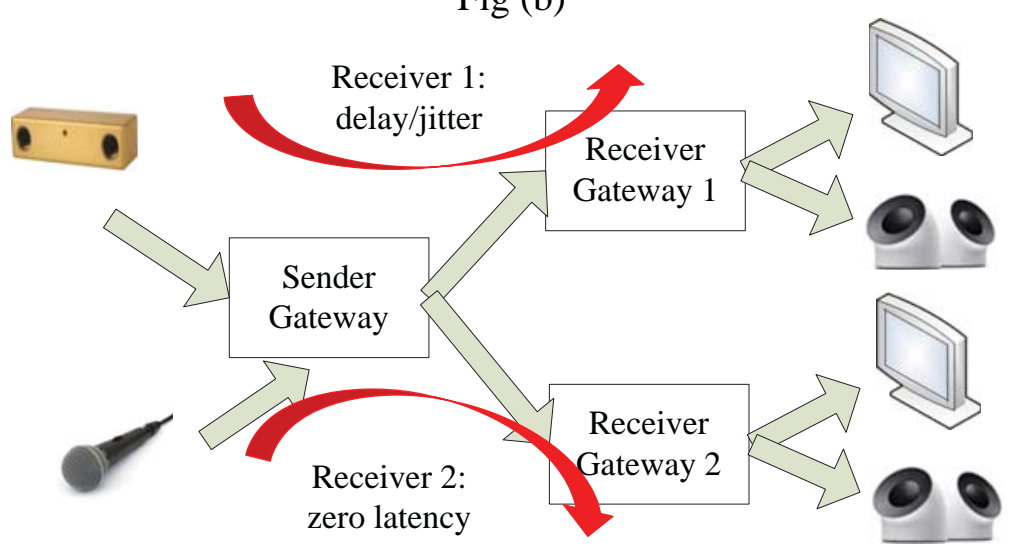

Fig (c)

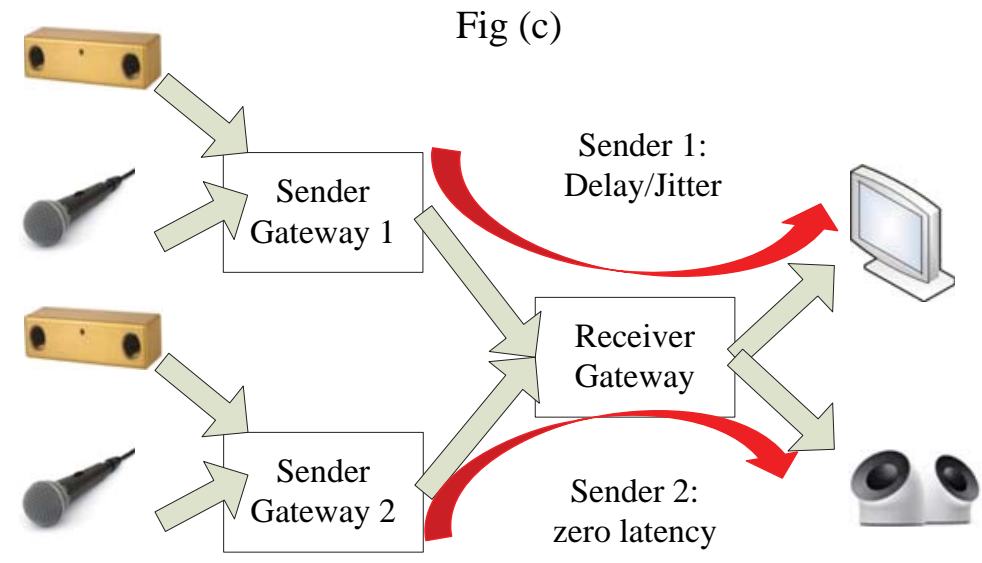

Figure 6.6: Three cases for synchronization evaluation in real teleimmersion. 

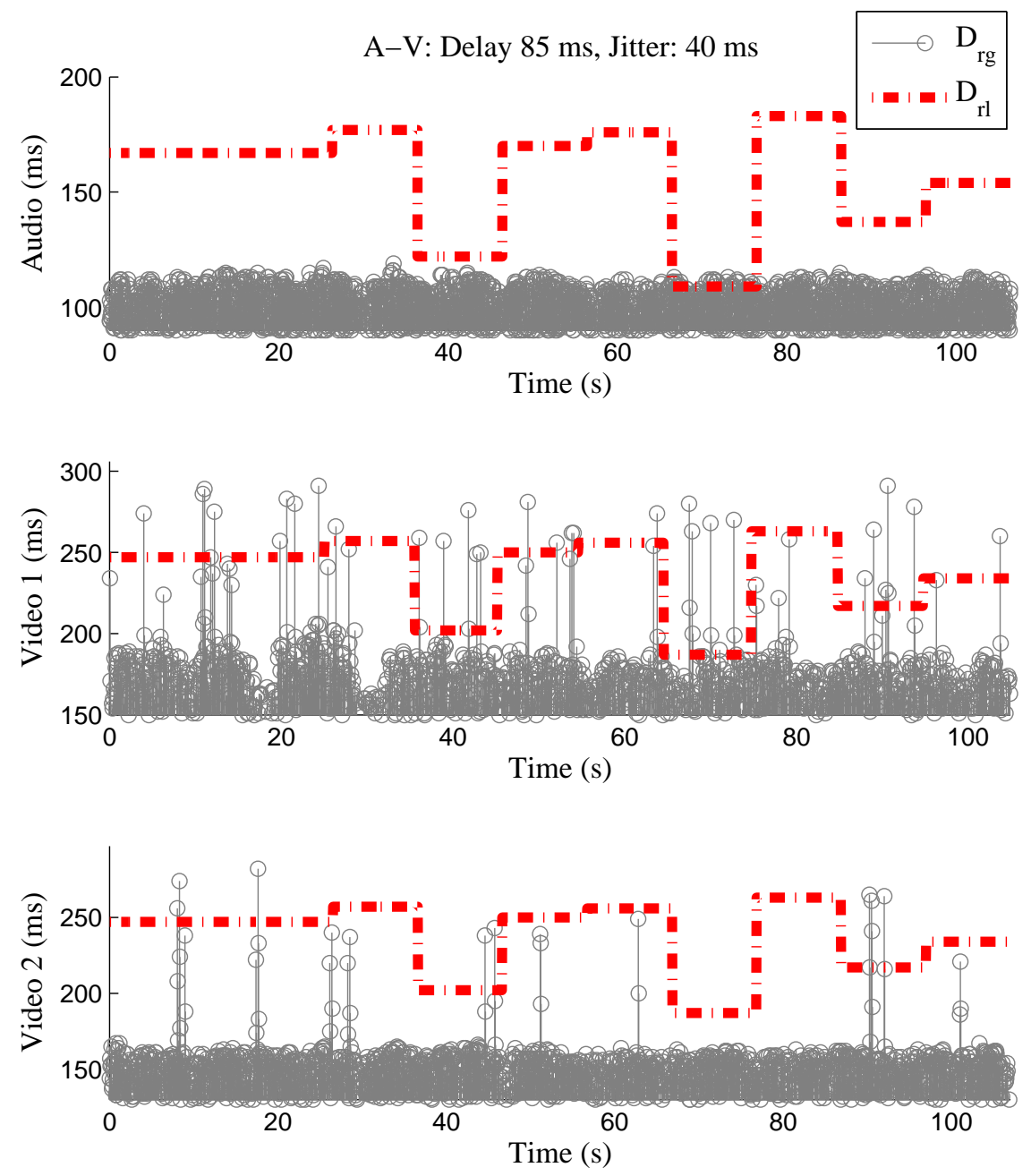

Figure 6.7: Case 1: two-party synchronization evaluation. Audio/video traffics with an average delay of 85 $\mathrm{ms}$ and jitter of $40 \mathrm{~ms}$.

a media frame experiences before it arrives at the receiver gateway) and $D_{r l}$ (i.e., the latency that a media frame experiences before it is released by the receiver gateway). We present the resulting intra-media, intrabundle and intra-session synchronization skews in Table. 6.2, 6.3 and 6.4. In our evaluations, we assume that the video modality $m_{V}$ is always the reference modality (intra-bundle synchronization reference), and that the video stream $1 s_{V, 1}$ is always the reference stream (intra-media synchronization reference). We set in TBuffer $\delta_{1}\left({ }^{\prime} \mathrm{A}\right.$ ') $=\delta_{1}\left({ }^{\prime} \mathrm{V}\right.$ ') $=0 \mathrm{~ms}$ (Eqn. 4.7), $\delta_{2}\left({ }^{\prime} \mathrm{A}\right.$ ', $\left.*\right)=\delta_{2}\left({ }^{\prime} \mathrm{A}\right.$ ', ' $\mathrm{V}$ ') $=80 \mathrm{~ms}$ (Eqn. 4.8), and $\mathrm{UR}_{\mathrm{TH}}\left({ }^{\prime} \mathrm{A}\right.$ ') $=\mathrm{UR}_{\mathrm{TH}}\left({ }^{\prime} \mathrm{V}^{\prime}\right)=0.98$ (Eqn. 4.6).

The figures and tables show that our synchronization implementation can successfully bound the synchronization skews within each media bundle (i.e., intra-stream, intra-media and intra-bundle synchroniza- 
Table 6.2: Case 1: $D_{r g}$ and $D_{r l}$ of two video and one audio streams, as well as the resulting skews. Media data are sent from site $n^{x_{0}}$ to site $n^{y_{0}} . \Delta D_{r l}\left(m_{A}^{x_{0}}, n^{y_{0}}\right)$ : intra-bundle (audio-visual) skew, $\Delta D_{r l}\left(s_{V, 2}^{x_{0}}, n^{y_{0}}\right)$ : intra-media (video) skew. Unit: ms.

\begin{tabular}{|c|ccccccccc|}
\hline Update & 1 & 2 & 3 & 4 & 5 & 6 & 7 & 8 & 9 \\
\hline \hline$D_{r l}\left(s_{A, 1}^{x_{0}}, n^{y_{0}}\right)$ & 167 & 177 & 122 & 170 & 176 & 109 & 183 & 137 & 154 \\
\hline$D_{r l}\left(s_{V, 1}^{x_{0}}, n^{y_{0}}\right)$ & 247 & 257 & 202 & 250 & 256 & 187 & 263 & 217 & 234 \\
\hline$D_{r l}\left(s_{V, 2}^{x_{0}}, n^{y_{0}}\right)$ & 247 & 257 & 202 & 250 & 256 & 187 & 263 & 217 & 234 \\
\hline$\left|\Delta D_{r l}\left(m_{A}^{x_{0}}, n^{y_{0}}\right)\right|$ & 80 & 80 & 80 & 80 & 80 & 78 & 80 & 80 & 80 \\
\hline$\left|\Delta D_{r l}\left(s_{V, 2}^{x_{0}}, n^{y_{0}}\right)\right|$ & 0 & 0 & 0 & 0 & 0 & 0 & 0 & 0 & 0 \\
\hline
\end{tabular}

Table 6.3: Case 2: $D_{r g}$ and $D_{r l}$ of one video stream and one audio stream from a single sender site $n^{x_{0}}$ to two receiver sites $n^{y_{0}}$ and $n^{y_{1}}$, as well as the resulting skews. $\Delta D_{r l}\left(m_{A}^{x_{0}}, n^{y_{0}}\right), \Delta D_{r l}\left(m_{A}^{x_{0}}, n^{y_{1}}\right)$ : intrabundle (audio-visual) skew. $n^{y_{1}}$ is the reference site, and $\Delta D_{r l}\left(u^{x_{0}}, n^{y_{0}}\right)$ is the intra-session skew. Unit: ms.

\begin{tabular}{|c|ccccccccc|}
\hline Update & 1 & 2 & 3 & 4 & 5 & 6 & 7 & 8 & 9 \\
\hline \hline$D_{r l}\left(s_{A, 1}^{x_{0}}, n^{y_{0}}\right)$ & 99 & 96 & 113 & 97 & 92 & 95 & 93 & 93 & 93 \\
\hline$D_{r l}\left(s_{V, 1}^{x_{0}}, n^{y_{0}}\right)$ & 179 & 176 & 193 & 177 & 172 & 175 & 173 & 173 & 173 \\
\hline$\left|\Delta D_{r l}\left(m_{A}^{x_{0}}, n^{y_{0}}\right)\right|$ & 80 & 80 & 80 & 80 & 80 & 80 & 80 & 80 & 80 \\
\hline$D_{r l}\left(s_{A, 1}^{x_{0}}, n^{y_{1}}\right)$ & 142 & 249 & 187 & 283 & 155 & 163 & 148 & 157 & 157 \\
\hline$D_{r l}\left(s_{V, 1}^{x_{0}}, n^{y_{1}}\right)$ & 222 & 329 & 267 & 363 & 235 & 243 & 228 & 237 & 237 \\
\hline$\left|\Delta D_{r l}\left(m_{A}^{x_{0}}, n^{y_{1}}\right)\right|$ & 80 & 80 & 80 & 80 & 80 & 80 & 80 & 80 & 80 \\
\hline$\left|\Delta D_{r l}\left(u^{x_{0}}, n^{y_{0}}\right)\right|$ & 43 & 153 & 74 & 186 & 63 & 68 & 55 & 64 & 64 \\
\hline
\end{tabular}

Table 6.4: Case 3: $D_{r g}$ and $D_{r l}$ of one video stream and one audio stream from two sender sites $n^{x_{0}}$ and $n^{x_{1}}$ to a single receiver site $n^{y_{0}}$, as well as the resulting skews. $\Delta D_{r l}\left(m_{A}^{x_{0}}, n^{y_{0}}\right), \Delta D_{r l}\left(m_{A}^{x_{1}}, n^{y_{0}}\right)$ : intra-bundle (audio-visual) skew. $n^{x_{1}}$ is the reference site, and $\Delta D_{r l}\left(u^{x_{0}}, n^{y_{0}}\right)$ is the intra-session skew. Unit: ms.

\begin{tabular}{|c|ccccccccc|}
\hline Update & 1 & 2 & 3 & 4 & 5 & 6 & 7 & 8 & 9 \\
\hline \hline$D_{r l}\left(s_{A, 1}^{x_{0}}, n^{y_{0}}\right)$ & 142 & 146 & 163 & 146 & 143 & 146 & 144 & 144 & 144 \\
\hline$D_{r l}\left(s_{V, 1}^{x_{0}}, n^{y_{0}}\right)$ & 222 & 226 & 243 & 226 & 223 & 226 & 224 & 224 & 224 \\
\hline$\left|\Delta D_{r l}\left(m_{A}^{x_{0}}, n^{y_{0}}\right)\right|$ & 80 & 80 & 80 & 80 & 80 & 80 & 80 & 80 & 80 \\
\hline$D_{r l}\left(s_{A, 1}^{x_{1}}, n^{y_{0}}\right)$ & 218 & 217 & 187 & 203 & 205 & 186 & 187 & 211 & 188 \\
\hline$D_{r l}\left(s_{V, 1}^{x_{1}}, n^{y_{0}}\right)$ & 298 & 280 & 239 & 272 & 265 & 240 & 241 & 266 & 240 \\
\hline$\left|\Delta D_{r l}\left(m_{A}^{x_{1}}, n^{y_{0}}\right)\right|$ & 80 & 63 & 52 & 69 & 60 & 54 & 54 & 55 & 52 \\
\hline$\left|\Delta D_{r l}\left(u^{x_{0}}, n^{y_{0}}\right)\right|$ & 76 & 54 & 4 & 46 & 42 & 14 & 17 & 42 & 16 \\
\hline
\end{tabular}


Site 1: A-V: Delay 0 ms, Jitter: 0 ms
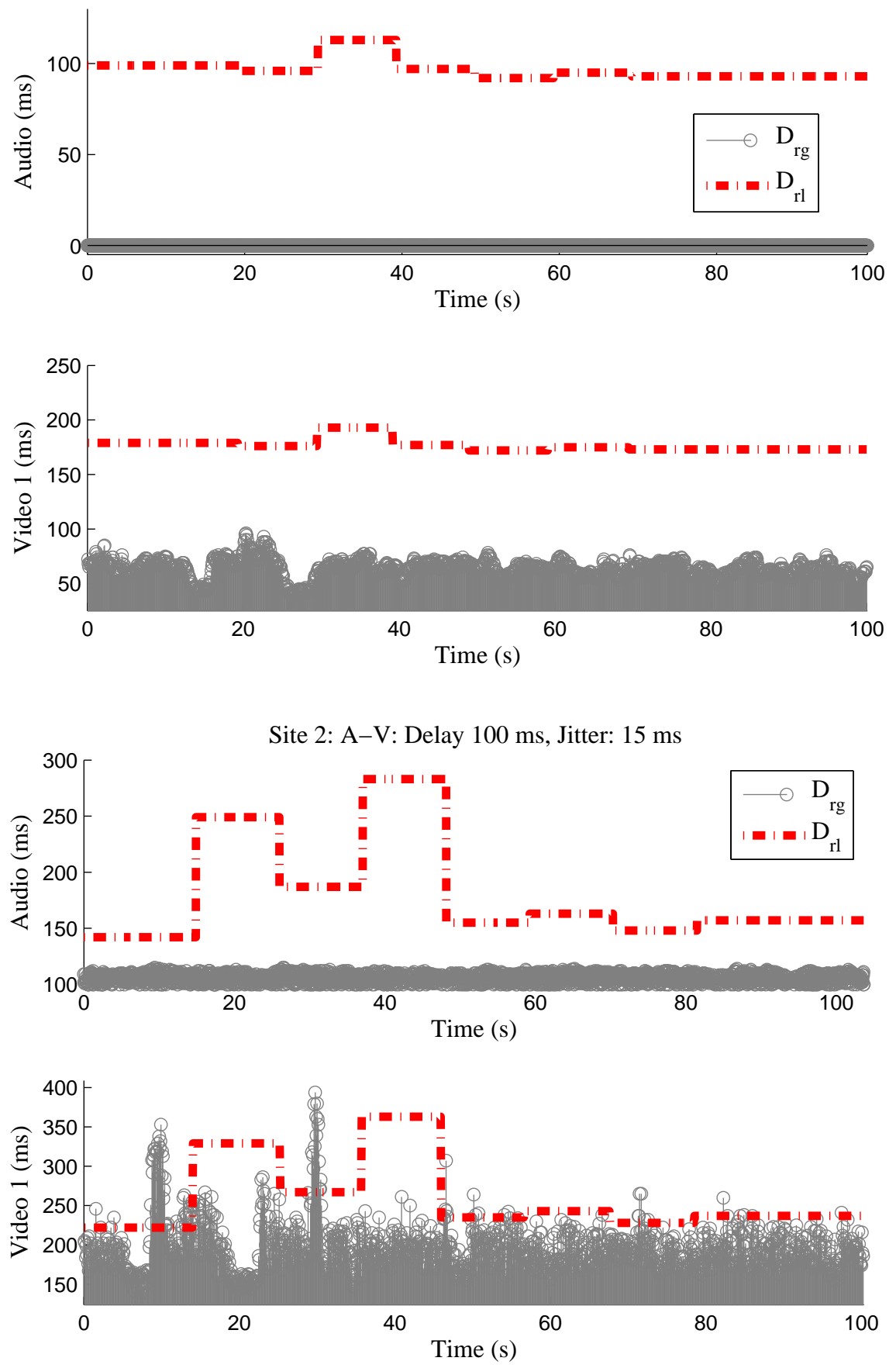

Figure 6.8: Case 2: inter-receiver synchronization evaluation. Receiver 1: audio/video traffics with no delay and jitter. Receiver 2: audio/video traffics with an average delay of $100 \mathrm{~ms}$ and jitter of $15 \mathrm{~ms}$. 
Site $1 \mathrm{~A}-\mathrm{V}$ : Delay $0 \mathrm{~ms}$, Jitter: $0 \mathrm{~ms}$
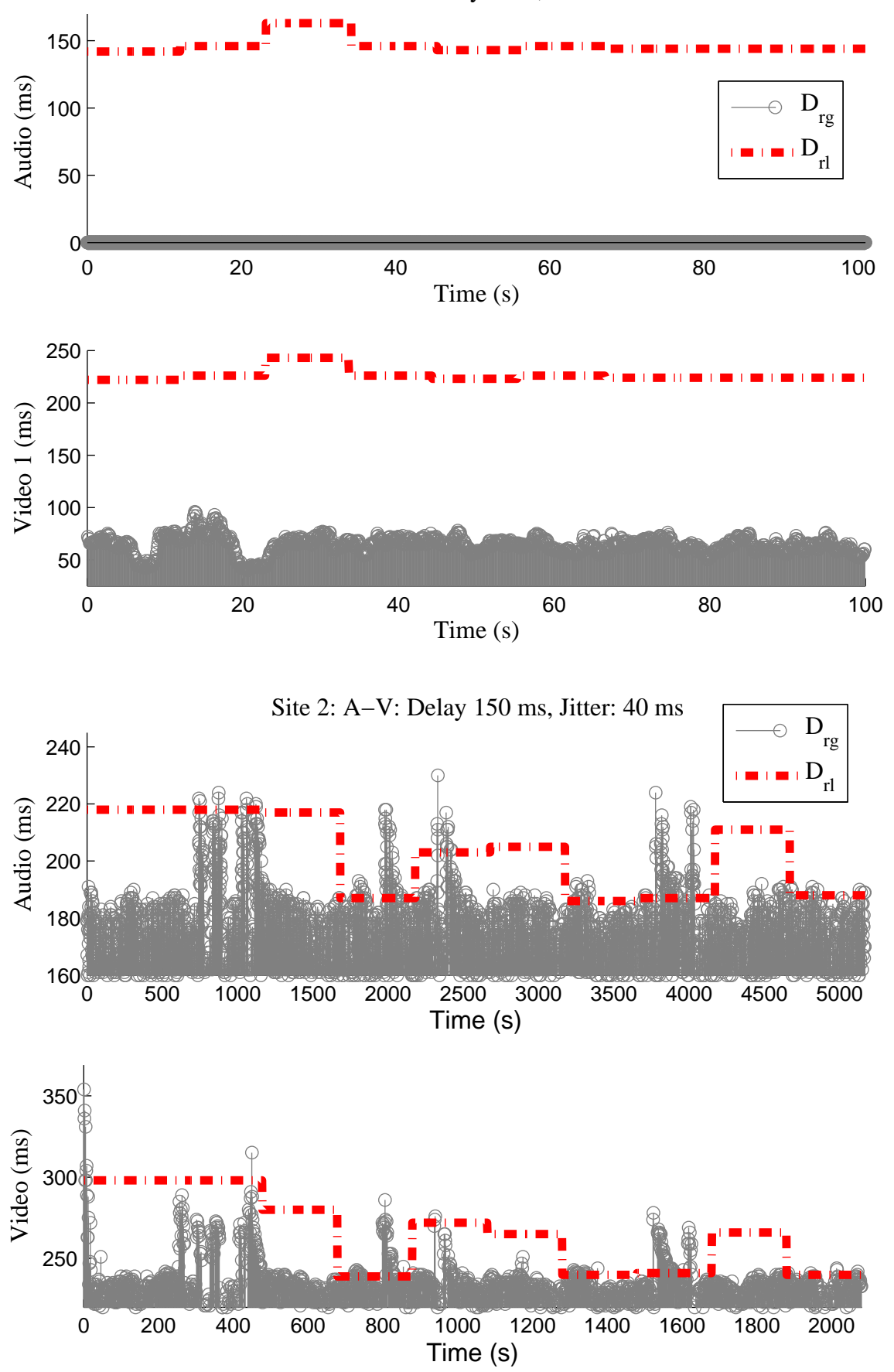

Figure 6.9: Case 3: inter-sender synchronization evaluation. Sender 1: audio/video traffics with no delay and jitter. Sender 2: audio/video traffics with an average delay of $150 \mathrm{~ms}$ and jitter of $40 \mathrm{~ms}$. 
tion). In terms of the intra-session synchronization, because we use a simplified centralized approach by offsetting the bundle delay heterogeneity based on the estimated one-way latencys, the resulting skews depend on the actual Internet jitter and the latency estimation accuracy. For example, Table. 6.3 shows the intra-session skews between the two receivers, which can be as much as $186 \mathrm{~ms}$ during the fourth update. By further observing Fig. 6.8, we find there is sudden jitter during the period of the third update, so the TBuffer algorithm has to preventively increase the buffer size (release time) in order to accommodate potential jitter. A solution to reduce the intra-session skew is to update presentation scheduling statuses more frequently and dynamically, depending on the network conditions.

\subsection{Summary}

In this chapter, we discuss the synchronization implementation details in teleimmersive applications. We propose a new RTP header in order to capture the time dependency and synchronization information across multi-media multi-stream bundle within the multi-site teleimmersive systems. Evaluation results demonstrate the performance of our synchronization framework. Limitations of the synchronization control approach in the existing implementation is also carefully discussed in our study.

\subsection{Table of Abbreviations and Notations}

\section{List of Abbreviations in Chapter 6:}

$\begin{array}{ll}\text { TEEVE } & \text { Teleimmersive Environment for EVErybody } \\ \text { NTP } & \text { Network Time Protocol } \\ \text { PTP } & \text { Precision Time Protocol } \\ \text { TCP } & \text { Transmission Control Protocol } \\ \text { UDP } & \text { User Datagram Protocol } \\ \text { IP } & \text { Internet Protocol } \\ \text { RTP } & \text { Real-time Transport Protocol } \\ \text { RTCP } & \text { Real-time Transport Control Protocol } \\ \text { MPS } & \text { Media Presentation Scheduling }\end{array}$




\section{List of Notations in Chapter 6:}

$i \quad$ Index of media modality

$k \quad$ Index of media frame

$t_{a r} \quad$ Arrival time at the sender producer wrapper

$t_{e} \quad$ Actual arrival time at the encoding machine

$T_{\text {cap }} \quad$ Encoding overhead/Duration at the capturing tier

$T_{\text {net }} \quad$ Duration at the distribution tier

$T_{\text {net }}^{\max } \quad$ Maximum distribution overhead within the session

tob $\quad$ Buffer time offset

$\delta_{1}(i) \quad$ Upper bound for intra-media synchronization skew of media modality $i$

$\delta_{2}(i, j) \quad$ Upper bound for intra-bundle synchronization skew between media modality $i$ and $j$

$\delta_{3} \quad$ Upper bound for intra-bundle synchronization skew within the session

$\mathrm{UR}_{\mathrm{TH}}(i) \quad$ Upper bound for unavailable rate for each sensory stream of media modality $i$

$D_{r g} \quad$ Latency of media data experienced before receiver gateway arrival

$D_{r l} \quad$ Latency of media data experienced before receiver gateway release

$\Delta D_{r l} \quad$ Multi-layer synchronization skew at due to $D_{r l}$ 


\section{CHAPTER 7}

\section{CONCLUSIONS AND FUTURE WORK}

\subsection{Conclusions}

This dissertation focuses on performing synchronized multi-modal media distribution in multi-site teleimmersion over the Internet. Multimedia synchronization is still an unsolved problem in teleimmersion and other next-generation multimedia systems, due to the diversity of Internet delays and computation/processing overhead incurred at multiple system locations, the emerging synchronization demands over large-scale multi-modal sensory streams from distributed media sensors, and the heterogeneity of end user interests in multi-functional interactive applications. The three issues represent the three most important orthogonal dimensions that concurrently dominate the overall synchronization problem. Existing research studies and industrial standards have a bias against one or multiple dimensions, so they are insufficient in addressing all aspects of the teleimmersive synchronization issues. In addition, previous synchronization control studies can create a tradeoff in balancing the media signal intelligibility and the interactive quality in real-time multimedia applications, and the human perceptual impact of the tradeoff cannot be captured by existing subjective metrics. Hence, it is difficult to design a synchronization control framework that is able to maximize the end user satisfactions.

In this dissertation, we present the synchronization problem and its interactions with human perception in Section 1. We complete a thorough survey in Section 2 demonstrating the insufficiency of existing studies on multimedia synchronization controls and the resulting subjective evaluations. We propose a new multi-dimensional classification model that systematically integrates the synchronization location, the synchronization demand and the activity-dependent synchronization reference hierarchy in Section 3, along with a decision algorithm for identifying the time correlations of multi-modal media frames captured at distributed sensory devices. In section 4 , we discuss a multi-tier collaborative synchronization control framework to meet different synchronization demands in all system locations. We enhance the control algorithm in Section 5 by proposing a subjective metric to capture the quality tradeoff of the interactive streaming 
media, conducting subjective evaluations based on the new metric, and user the real perceptual feedback to guide online synchronization adaptations. Section 6 describes our synchronization implementations in real teleimmersive systems.

We show in the evaluation results that we are able to identify the time correlations of multi-modal media frames and to consistently bound the synchronization skews throughout media computation, processing and distribution in teleimmersion. We demonstrate the heterogeneous perceptual demand of both conversational and rock-paper-scissor applications during the synchronization controls. We also show the effectiveness of our proposed subjective metric in capturing the perceptual tradeoff of the streaming media quality and in guiding the synchronization adaptations to achieve a teleimmersive system with the best subjective quality.

\subsection{Lessons Learned}

We have learned several lessons from our multimedia synchronization research study.

First, as next-generation multimedia systems are growing more complex in terms of the multi-location pipeline architecture, we have to resort to collaborative synchronization controls in all locations in order to prevent the propagation of the skews during the media computation and distribution, and to facilitate the media synchronization at their final presentation. In our study, we solve the collaborative control problem by bounding/minimizing the skews in each location independently. We do not manage to investigate the impact of skew propagation across multiple places, and to design adaptation algorithms based on this impact. This can allow our proposed control framework to become more configurable in diverse multimedia system settings, and to reduce the framework's own complexity which may otherwise increase exponentially as the number of control locations scales in the system.

Second, we rely on the estimated media computation (encoding) time and the Internet traffic patterns to guide the online synchronization control (e.g., CloudStream at the capturing tier and TBuffer at the presentation tier). The underlying assumption is that the future computation and Internet statistics can be predicted with reasonable accuracy, based upon the past and current records. However, under the circumstance of an unusually long encoding latency or an unexpected change of the Internet quality, the control adaptation algorithms may fail to respond timely, and an unbounded synchronization skew is still likely. Hence, our control algorithms are, in some sense, best-effort, and there is no better solution to guarantee perfect synchronization given the dynamic and uncontrollable real system issues.

Third, due to the complexity of interactions and tradeoffs among the media signal intelligibility, the interactive quality and the synchronization quality, it is very difficult to use a closed form to describe their 
combined impact. Hence, we have to resort to the machine learning approach to find the quality mapping as described in Section 5. Existing ITU standards try to present the closed form by simplifying the dependencies across different quality attributes, but the credibility of such simplification remain to be challenged.

\subsection{Future Work}

\subsubsection{Standardizing Synchronization Protocols}

As discussed in Section 2, we are unable to employ RTP and RTCP in our TEEVE testbed due to a lack of real-time support for synchronizing the time-correlated scalable multi-modal media data from multiple sender sites, and for specifying the synchronization reference dependencies during the online controls. Hence, in our teleimmersive implementation, we design our own proprietary protocols for multimedia distribution and synchronization. We foresee an urgent demand in extending existing RTP and RTCP protocols to feature new multi-modal multi-stream characteristics for next-generation interactive multimedia systems, and to offer both specification and control support for realizing the demands of multi-layer multimedia synchronization.

Specifically, we envision the following changes in RTCP:

- New control service component at the sender sites to identify the time-correlated media frames from different multi-modal sensory streams that belong to each synchronization point.

- New distributed control mechanism at the receiver sites to perform cooperative multi-device synchronization.

- New control support for inter-sender synchronization.

Accordingly, we modify the RTP header to provide the underlying specification support for RTCP:

- The timestamp field in the current RTP header must be extended to tell the synchronization point information. Multi-layer synchronization skews can be computed during the media distribution, and existing RTP mixing functionality can also be improved by performing over the identified synchronization point sequence.

- The synchronization reference dependencies must also be indicated in the header, so that the most important media data can be given the priority during QoS adaptations at different network end devices. 


\subsubsection{Mobile Synchronization}

Our synchronization control framework are mainly designed for wireline and wireless 802.11 network. With the blossoms of mobile devices operating on $3 \mathrm{G} / 4 \mathrm{G}$ cellular network, multiple issues can emerge which will impede the multimedia synchronization.

- Network unreliability. Existing synchronization algorithms (e.g. bandwidth resource allocation and presentation scheduling) usually perform by estimating the network conditions based on the observations of previous media traffic patterns. Owing to the uncontrollable interferences between the base stations (access points) and end users, the cellular connections are more prone to jitter and losses, and the resulting data traffic can demonstrate huge burstiness. This leads to a very poor prediction accuracy of the cellular network quality, which can tremendously hinder the resulting synchronization performance. New control study should be proposed to address the issue of cellular network unreliability and dynamics.

- Device mobility. The mobility of end devices can create two problems. First, the frequent join/leave of the users and the resulting changes of relay paths options make it impossible to develop a stable SyncCast-like multicast overlay. Each user may only request the media data from reachable neighbors at the best effort, causing unconstraint multi-layer synchronization skews. Second, end devices can switch among multiple base stations and access points during their movement, and important synchronization information can be dropped at each handover. This demands a fast resynchronization mechanism to recover the out-of-sync period.

- New perceptual demands of users. The current technological trend foresees an increasing number of new multi-modal sensors co-equipped on a single mobile device, and their broad usage along with new synchronization demands in diverse multimedia applications. End users can exhibit heterogeneous perceptual demands on synchronizing these multi-modal sensory data in different applications, and subjective evaluations are the only approach to understand real human perceptual quality.

To sum up, we believe that the multimedia synchronization will continue to be an important problem in the future as our multimedia environments are getting richer in terms of multi-modal devices, faster and more ubiquitous in terms of the network inter-connectivity, and more versatile in terms of their usages in broad multimedia applications. This dissertation well serves as a pioneering work to encourage the community to rethink of synchronization models, concepts, specifications, mechanisms and protocols. We will continue to see new synchronization studies and industrial standards in the near future. 


\subsection{Table of Abbreviations and Notations}

List of Abbreviations in Chapter 7:

TEEVE Teleimmersive Environment for EVErybody

RTP Real-time Transport Protocol

RTCP Real-time Transport Control Protocol

QoS Quality of Service 


\section{REFERENCES}

[1] Z. Huang, W. Wu, K. Nahrstedt, A. Arefin, and R. Rivas, "Tsync: A new synchronization framework for multi-site 3D tele-immersion," in Proc. ACM Workshop on Network and Operating Systems Support for Digital Audio and Video, Jun. 2010.

[2] “Cisco Telepresence. Cisco Corporation." http://www.cisco.com.

[3] "Skype." http://www.skype.com.

[4] "Vidyo Telepresence. Vidyo corporation." http://www.vidyo.com.

[5] P. Bajcsy, K. McHenry, H.-J. Na, R. Malik, and et al, "Immersive environments for rehabilitation activities," in Proc. of ACM Int'l Conference on Multimedia, 2009, pp. 829-832.

[6] M. Forte and G. Kurillo, "Cyberarchaeology - experimenting with teleimmersive archaeology," in Proc. of Int'l Conference on Virtual Systems and Multimedia, 2010.

[7] T. D. C. Little and A. Ghafoor, "Spatio-temporal composition of distributed multimedia objects for value-added networks," IEEE Computer, vol. 24, no. 10, pp. 42-50, 1991.

[8] T. Meyer, W. Effelsberg, and R. Steinmetz, "A taxonomy on multimedia synchronization," in Proceedings of IEEE Workshop on Future Trends of Distributed Computing Systems, Sep. 1994, pp. 97-103.

[9] G. Blakowski and R. Steinmetz, "A media synchronization survey: Reference model, specification, and case studies," IEEE Journal on Selected Areas in Communications, vol. 14, no. 1, pp. 5-35, 1996.

[10] R. Vasudevan, E. Lobaton, G. Kurillo, and et al, "A methodology for remote virtual interaction in teleimmersive environments," in Proceedings of ACM International Conference on Multimedia System, 2010, pp. 281-292.

[11] Speex, “Speex: A free codec for free speech.” [Online]. Available: http://www.speex.org/

[12] Z. Yang, W. Wu, K. Nahrstedt, G. Kurillo, and R. Bajcsy, "Enabling multi-party 3d tele-immersive environments with viewcast," ACM Transactions on Multimedia Computing, Communications, and Applications, vol. 6, no. 2, 2010.

[13] E. Biersack, W. Geyer, and C. Bernhardt, "Intra- and inter-stream synchronization for stored multimedia systems," in Proc. of IEEE Int'l Confernce on Multimedia Computing and Systems, 1996, pp. 372-381.

[14] Y. Ishibashi, T. Kanbara, and S. Tasaka, "Inter-stream synchronization between haptic media and voice in collaborative virtual environments," in Proc. of ACM Int'l Conference on Multimedia, 2004. 
[15] H. Liu and M. Zarki, "An adaptive delay and synchronization control scheme for Wi-Fi based audio/video conferencing," Springer Wireless Networks, vol. 12, no. 4, pp. 511-522, Jul. 2006.

[16] C. Diot and L. Gautier, "A distributed architecture for multiplayer interactive applications on the Internet," IEEE Network, vol. 13, no. 4, pp. 6-15, 1999.

[17] C. Liu, Y. Xie, M. Lee, and T. Saadawi, "Multipoint multimedia teleconference system with adaptive synchronization," IEEE Journal of Selected Areas in Communication (JSAC), vol. 14, no. 7, pp. 1422-1435, 1996.

[18] R. Steinmetz and K. Nahrstedt, "Multimedia computing, communications and applications, Prentice Hall, 1995."

[19] J. L. F. Boronat and M. Garcia, "Multimedia group and inter-stream synchronization techniques: a comparative study," Information Systems, no. 34, pp. 108-131, 2009.

[20] R. Steinmetz, "Human perception of jitter and media synchronation," IEEE Journal on Selected Areas in Communications, vol. 14, no. 1, pp. 61-72, 1996.

[21] I. Curcio and M. Lundan, "Human perception of lip synchronization in mobile environment," in Proc. of IEEE Int'l Symposium on a World of Wireless, Mobile and Multimedia Networks, 2007.

[22] ITU-G.1070, "Opinion model for video-telephony applications," 2007. [Online]. Available: http://www.itu.int/rec/T-REC-G.1070/en/

[23] T. Little, "A framework for synchronous delivery of time-depdent multimedia data," Multimedia System, vol. 1, no. 2, pp. 87-94, 1993.

[24] L. Ehley, B. Furth, and M. Ilyas, "Evaluation of multimedia synchronization techniques," in Proceedings of Int'l Conference on Multimedia Computing and Systems, 1994, pp. 110-119.

[25] C.-M. Huang, C.-W. Lin, C.-C. Yang, C.-H. Chang, and H.-H. Ku, "An SVC-MDC video coding scheme using the multi-core parallel programming paradigm for $\mathrm{P} 2 \mathrm{P}$ video streaming," in Proc. of IEEE Int'l Conf. on Computational Sciences and Its Applications, 2009.

[26] Y.-L. Huang, Y.-C. Shen, and J.-L. Wu, "Scalable computation for spatially scalable video coding using NVIDIA CUDA and multi-core CPU," in Proc. of ACM Int'l Conference on Multimedia, Oct. 2009, pp. 361-370.

[27] E. Brosh, A. Levin, and Y. Shavitt, "Approximation and heuristic algorithms for minimum-delay application-layer multicast trees," IEEE/ACM Transaction on Networking, vol. 15, no. 2, pp. 473484, 2007.

[28] S. Khuller, B. Raghavachari, P. State, and N. Young, "Balancing minimum spanning trees and shortest-path trees," Algorithmica, vol. 14, pp. 305-321, 1995.

[29] J. Kruskal, "On the shortest spanning subtree of a graph and the traveling salesman problem," American Mathematical Society, vol. 7, no. 1, pp. 48-50, 1956.

[30] G. N. Rouskas and I. Baldine, "Multicast routing with end-to-end delay and delay variation constraints," IEEE Journal on Selected Areas in Communications, vol. 15, no. 3, pp. 346-356, Apr. 1997. 
[31] R. Zimmermann and K. Liang, "Spatialized audio streaming for networked virtual environments," in Proc. of ACM Int'l Conference on Multimedia, 2008, pp. 299-308.

[32] M. Chen, "Low-latency lip-synchronized videoconferencing system," in Proceedings of Conference on Human Factors in Computing Systems, 2003, pp. 465-471.

[33] L. Qiao and K. Nahrstedt, "Lip synchronizatioin within an adaptive VoD," in Proceedings of SPIE Multimedia Computing and Network, 1997, pp. 170-181.

[34] ITU-BT.500, "Methodology for the subjective assessment of the quality of television pictures," 2002.

[35] ITU-P.910, "Subjective video quality assessment methods for multimedia applications," 2008.

[36] B. Sat and B. W. Wah, "Statistical scheduling of offline comparative subjective evaluations for realtime multimedia," IEEE Transaction on Multimedia, vol. 11, no. 6, pp. 1114-1130, Oct. 2009.

[37] Z. Huang, “The design of a multi-party VoIP conferencing system," M.S. thesis, University of Illinois at Urbana-Champaign, Urbana, IL, 2009.

[38] A. Khan, L. Sun, E. Jammeh, and E. Ifeachor, "Quality of experience-driven adaptation scheme for video applications over wireless networks," IET Communications, 2010.

[39] L. Sun and E. Ifeachor, "Voice quality prediction models and their application in VoIP networks," IEEE Communications, vol. 3, pp. 1478-1483, 2004.

[40] B. Sat and B. Wah, "Playout scheduling and loss-concealments in VoIP for optimizing conversational voice communication quality," in Proceedings of ACM Int'l Conference on Multimedia, Sep. 2007, pp. 137-146.

[41] C. C. Chang and C. J. Lin, "LIBSVM: A library for Support Vector Machines.” [Online]. Available: http://www.csie.ntu.edu.tw/ cjlin/libsvm/

[42] P. Agarwal, R. Rivas, W. Wu, A. Arefin, Z. Huang, and K. Nahrstedt, "SAS kernel: Streaming as a service kernel for correlated multi-streaming," in Proc. ACM Workshop on Network and Operating Systems Support for Digital Audio and Video, 2010.

[43] A. G. Bell, "US Patent 174,465: Improvement in Telegraphy,” Mar 7, 1876.

[44] C. Basilio, "Antonio meucci inventore del telefono," Notiziario Tecnico Telecom Italia, vol. 12, no. 1, p. 114, 2003.

[45] G. Graham, Canadian film technology, pp. 41-43, 1989.

[46] “The picture of the future," Bell Labs Record, vol. 47, pp. 134-186, 1969.

[47] U. Michel, "History of acoustic beamforming," in Proceedings of Berlin Beamforming Conference, 2006.

[48] "Kesmai corporation," http://www.mobygames.com/company/kesmai-corporation/.

[49] B. Blesser, "Digitization of audio: a comprehensive examination of theory, implementation, and current practice," Journal of the Audio Engineering Society, vol. 26, pp. 739-771, Oct. 1978. 
[50] T. Stockham, "A/D and D/A converters: their effect on digital audio fidelity," Digital Signal Processing, IEEE Press, pp. 55-66, 1972.

[51] PictureTel, "Picturetel In Project With I.B.M., New York Times," 1991.

[52] S. Casner, K. Seo, W. Edmond, and C. Topolcic, "N-way conferencing with packet video," in Proceedings of International Workshop on Packet Video, 1990.

[53] B. Gardner, "A realtime multichannel room simulator," 1992.

[54] N. A. Streitz, J. Geibler, J. M. Haake, and J. Hol, "DOLPHIN: Integrated meeting support across liveboards, local and remote desktop environments," in Proceedings of ACM International on Computer Supported Cooperative Work, 1994, pp. 345-358.

[55] “Cornell University, The CU-SeeMe Project,” http://ipsix.org/source/cuseeme project.html.

[56] “The Cambridge iTV Trial, 1994-1996," ATM Ltd and Online Media. [Online]. Available: http://koo.corpus.cam.ac.uk/projects/itv/

[57] M. Hodges, R. Sasnett, and M. Ackerman, "Athena Mouse: a construction set for multimedia applications,” IEEE Software, pp. 37-43, Jan. 1989.

[58] R. Steinmetz, "Analyse von synchronisation mechanismen mit anwendung im multimedia-bereich," in Proceedings of GI ITG Workshop Sprachen und System zur Parallelverarbeitung, Jan. 1990, pp. $39-47$.

[59] T. Wahl and K. Rothernel, "Representing time in multimedia systems," in Proceedings of IEEE International Conference on Multimedia Computing and Systems, May 1994, pp. 538-543.

[60] C. Hamblin, "Instants and intervals," in Proceedings of 1st Conference of the International Society for the Study of Time, 1972, pp. 324-331.

[61] J. Allen, "Maintaining knowledge about temporal intervals," ACM Communications, vol. 26, no. 11, pp. 832-843, 1983.

[62] R. Steinmetz and C. Engler, "Human perception of media synchronization," Technical Report 43.9310, IBM European Networking Center Heidelberg, 1993.

[63] R. Dannenberg and R. Stern, "Experiments concerning the allowable skew of two audio channels operating in the stereo mode," Personal Communications, 1993.

[64] D. P. Anderson and G. Homsy, "A continuous media I/O server and its synchronization mechanism," IEEE Computer, vol. 24, no. 10, pp. 51-57, 1991.

[65] R. Yavatkar, "MCP: A protocol for coordination and temporal synchronization in collaborative applications," in Proceedings of the IEEE International Conference Distributed Computing Systems, Jun. 1992, pp. 606-613.

[66] K. Ravindran and V. Bansal, "Delay compensation protocols for synchronization of multimedia data streams," IEEE Transaction on Knowledge Data Engineering, vol. 4, no. 5, pp. 574-589, 1993.

[67] M. Woo, N. Qazi, and A. Ghafoor, "A synchronization framework for communication of preorchestrated multimedia information," IEEE Network, vol. 1, no. 8, pp. 52-61, 1994. 
[68] K. Rothermel and T. Helbig, "An adaptive stream synchronization protocol," in Proceedings of ACM International Workshop on Network and Operating System Support for Digital Audio and Video, 1995, pp. 189-202.

[69] K. Cluver and P. Noll, "Reconstruction of missing speech frames using sub-band excitation," in Proceedings of IEEE International Symposium on Time-Frequency and Time-Scale Analysis, Jun. 1996, pp. 277-280.

[70] N. Hu and P. Steenkiste, "Estimating available bandwidth using packet pair probing," in Technical Report CMU-CS-02-166, 2002.

[71] S. Ramanathan and P. V. Rangan, "Continuous media synchronization in distributed multimedia systems," in Proceedings of ACM International Workshop on Network and Operating System Support for Digital Audio and Video, Nov. 1992, pp. 289-296.

[72] S. Ramanathan and P. Rangan, "Feedback techniques for intra-media continuity and inter-media synchronization in distributed media systems," Computer Journal, vol. 36, no. 1, pp. 19-31, 1993.

[73] P. N. Zarros, M. J. Lee, and T. N. Saadawi, "Interparticipant synchronization in real-time multimedia conferencing using feedback," IEEE/ACM Transaction on Networking, vol. 4, no. 2, pp. 173-180, 1996.

[74] K. Rothermel and T. Helbig, "An adaptive protocol for synchronizing media streams," ACM/Springer Multimedia System Journal, vol. 5, no. 5, pp. 324-336, 1997.

[75] S. Y. Tov, "Happy 10th birthday, VoIP,” The Marker, Jun, 2005.

[76] B. Damer, “Avatars: exploring and building virtual worlds on the Internet," Peachpit Press, pp. 383386, 1998.

[77] “Caltech/CERN Project,” http://pcbunn.cithep.caltech.edu/.

[78] ITU-H.263, "Video coding for low bit rate communication," 2005. [Online]. Available: http://www.itu.int/rec/T-REC-H.263/en/

[79] ITU-H.323, "Packet-based multimedia communications systems," 2009. [Online]. Available: http://www.itu.int/rec/T-REC-H.323/en/

[80] RFC-1889, “Obsolete version - RTP: A transport protocol for real-time applications," Jan. 1996. [Online]. Available: http://tools.ietf.org/html/rfc1889/

[81] "ISO/IEC 14496-1: Coding of audio-visual objects," 2010. [Online]. Available: http://www.iso.org/

[82] I. F. Akyildiz and W. Yen, "Multimedia group synchronization protocols for integrated services networks," IEEE Journal of Selected Areas of Communications, vol. 14, no. 1, pp. 162-173, 1996.

[83] Y. Ishibashi, A. Tsuji, and S. Tasaka, "A group synchronization mechanism for stored media in multicast communications," in Proceedings of Annual Joint Conference of the IEEE Computer and Communications Societies, Apr. 1997, pp. 692-700.

[84] Y. Ishibashi and S. Tasaka, "A distributed control scheme for group synchronization in multicast communications," in Proceedings of International Symposium Communications, Nov. 1999, pp. 317323. 
[85] Y. Ishibashi and S. Tasaka, "A group synchronization mechanism for live media in multicast communications," in Proceedings of IEEE Global Telecommunications Conference, Nov. 1997, pp. 746-752.

[86] M. Parsa, Q. Zhu, and J. J. Garcia-Luna-Aceves, "An iterative algorithm for delay-constrained minimum-cost multicasting," IEEE/ACM Transaction on Networking, vol. 6, pp. 461-474, 1998.

[87] S. Chen and K. Nahrstedt, "An overview of quality-of-service routing for the next generation highspeed networks: Problems and solutions," IEEE Network, vol. 12, no. 6, pp. 64-79, Nov. 1998.

[88] B. Knutsson, M. M. Games, H. Lu, W. Xu, and B. Hopkins, "Peer-to-Peer support for massively multiplayer games," in Proceedings of IEEE International Conference on Computer Communications, Mar. 2004.

[89] T. Iimura, "Zoned federation of game servers: A peer-to-peer approach to scalable multi-player online games," in Proceedings of ACM NetGame, 2004, pp. 116-120.

[90] X. Zhang, J. Liu, B. Li, and T. shing Peter Yum, "Coolstreaming/donet: A data-driven overlay network for peer-to-peer live media streaming," in Proceedings of IEEE International Conference on Computer Communications, Mar. 2005, pp. 2102-2111.

[91] "IEEE 802.11 standard: LAN/MAN wireless LANS," 2008. [Online]. Available: http: //standards.ieee.org/getieee802/802.11.html

[92] S. Marcheschi, O. Portillo, M. Raspolli, C. Avizzano, and M. Bergamasco, "The haptic desktop: a novel 2D multimodal device," in Proceedings of IEEE International Conference on Robot and Human Interactive Communication, Sep. 2004, pp. 521-526.

[93] B. Baxter, V. Scheib, M. C. Lin, and D. Manocha, "DAB: Interactive haptic painting with 3D virtual brushes," in In Proceedings of ACM SIGGRAPH, 2001, pp. 461-468.

[94] B. Lo, S. Thiemjarus, R. King, and G. Yang, "Body sensor network - a wireless sensor platform for pervasive healthcare monitoring," in In Proceedings of IEEE International Conference on Pervasive Computing, May 2005.

[95] R. E. Mayagoitia, A. V. Nene, and P. H. Veltink, "Accelerometer and rate gyroscope measurement of kinematics: an inexpensive alternative to optical motion analysis systems," Journal of Biomechanics, vol. 35, no. 4, pp. 537-542, 2002.

[96] T. Do, K. A. Hua, and M. Tantaoui, "P2VoD: Providing fault tolerant video-on-demand streaming in peer-to-peer environment," in Proceedings of IEEE International Conference on Communications, Jun. 2004.

[97] X. Liao, H. Jin, Y. Liu, L. M. Ni, and D. Deng, “AnySee: Peer-to-Peer live streaming,” in Proceedings of IEEE International Conference on Computer Communications, Apr. 2006.

[98] M. Zhang, L. Sun, X. Xi, and S. Yang, "iGridMedia: The system to provide low delay peer-to-peer live streaming service over internet," in Proceedings of IEEE International Conference on Global Telecommunications, Nov. 2008, pp. 1-5.

[99] T. Nunome and S. Tasaka, "Inter-destination synchronization quality in a multicast mobile ad hoc network," in Proceedings of IEEE International Symposium on Personal, Indoor and Mobile Radio Communications, Sep. 2005. 
[100] A. Boukerche, S. Hong, and T. Jacob, "An efficient synchronization scheme of multimedia streams in wireless and mobile systems," IEEE Transaction on Parallel and Distributed Systems, vol. 13, no. 9, pp. 911-923, 2002.

[101] S. Tasaka, Y. Ishibashi, and M. Hayashi, "Inter-destination synchronization quality in an integrated wired and wireless network with handover," in Proceedings of IEEE International Conference on Global Telecommunications, 2002, pp. 1560-1565.

[102] A. Boukerche, S. Hong, and T. Jacob, "Synchronization and handoff management schemes for wireless multimedia systems," International Journal on Computer Telecommunicaiton Networking, vol. 41, no. 3, pp. 347-362, 2003.

[103] A. Boukerche and H. Owens, "Media synchronization and qos packet scheduling algorithms for wireless systems," Mobile Networks and Applications, vol. 10, no. 1, pp. 233-249, 2005.

[104] K. Hikichi, H. Morino, I. Arimoto, K. Sezaki, and Y. Yasuda, "The evaluation of delay jitter for haptics collaboration over the internet," in Proceedings of IEEE Global Telecommunications Conference, 2002, pp. 1492-1496.

[105] Y. Ishibashi, T. Hasegawa, and S. Tasaka, "Group synchronization control for haptic media in networked virtual environments," in Proceedings of International Symposium on Haptic Interfaces for Virtual Environment and Teleoperator Systems, 2004, pp. 106-113.

[106] S. Firestone, T. Ramalingam, and S. Fry, "Voice and video conferencing fundamentals, CISCO Press, 2007."

[107] X. Li, Y. Cui, and Y. Xue, "Towards an automatic parameter-tuning framework for cost optimization on video encoding cloud," International Journal of Digital Multimedia Broadcasting, 2012.

[108] Y. Ding, J. Liu, and S. Lian, "Multi-stream 3D video distribution over peer-to-peer networks," Signal Processing: Image Communication, p. 470C483, Feb. 2012.

[109] P. Leroux, V. Verstraete, F. De Turck, and P. Demeester, "Synchronized interactive services for mobile devices over ipdc/dvb-h and umts," in Proceedings of IEEE/IFIP International Workshop on Broadband Convergence Networks, May 2007, pp. 1-12.

[110] F. Boronat, J. C. G. Cebollada, and J. L. Mauri, "Study of delay jitter with and without peak rate enforcement," Journal of Multimedia Tools and Applications, vol. 40, no. 2, pp. 285-319, Nov. 2008.

[111] C. Perkins and T. Schierl, "Rapid synchronisation of RTP flows," Nov. 2010. [Online]. Available: http://tools.ietf.org/html/rfc6051

[112] R. van Brandenburg, H. Stokking, O. van Deventer, F. Boronat, and et al, "RTCP for inter-destination media synchronization," Jun. 2012. [Online]. Available: https://tools.ietf.org/html/ draft-ietf-avtcore-idms-05

[113] I. Vaishnavi, D. Bulterman, P. Cesar, and B. Gao, "Media presentation synchronisation for nonmonolithic rendering architectures," in Proceedings of IEEE International Symposium on Multimedia Workshops, 2007, pp. 181-186.

[114] J. Yun, H. Lee, and K. Park, "Orchestral media: The method for synchronizing single media with multiple devices for ubiquitous home media services," in Proceedings of IEEE International Conference on Convergence and Hybrid Information Technology, 2008, pp. 340-345. 
[115] M. Rautiainen, H. Aska, T. Ojala, M. Hosio, and et al, "Swarm synchronization for multi-recipient multimedia streaming," in Proceedings of IEEE International Conference on Multimedia and Expo, 2009, pp. 786-789.

[116] K. Tasaka, N. Imai, M. Isomura, and A. Idoue, "A media synchronization method for real-time group communication in a multiple device environment," in Proceedings of IEEE International Conference on Intelligence in Next Generation Networks, 2009, pp. 1-6.

[117] "IEEE 1588 standard: Precise time synchronization as the basis for real time applications in automation," 2008. [Online]. Available: http://www.ieee1588.com/

[118] T. Fujimoto, Y. Ishibashi, and S. Sugawara, "Influences of inter-stream synchronization error on collaborative work in haptic and visual environments," in Proceedings of IEEE Symposium on Haptic Interfaces for Virtual Environment and Teleoperator System, 2008, pp. 113-119.

[119] G. Ghinea and O. A. Ademoye, "Perceived synchronization of olfactory multimedia," IEEE Transactions on Systems, Man, and Cybernetics, vol. 40, no. 4, pp. 657-663, 2010.

[120] S. Hoshino, Y. Ishibashi, N. Fukushima, and S. Sugawara, "Qoe assessment in olfactory and haptic media transmission: Influence of inter-stream synchronization error," in Proceedings of IEEE International Workshop on Communications Quality and Reliability, 2011, pp. 1-6.

[121] L. Goldmann, J.-S. Lee, and T. Ebrahimi, "Temporal synchronization in stereoscopic video: Influence on quality of experience and automatic asynchrony detection," in Proceedings of IEEE International Conference on Image Processing, Sep. 2010, pp. 3241-3244.

[122] J. Beerends, C. De, and E. Frank, "The influence of video quality on perceived audio quality and vice versa," Journal of Audio Engineering Society, vol. 47, no. 5, pp. 355-362, May 1999.

[123] O. Daly-Jones, A. Monk, and L. Watts, "Some advantages of video conferencing over high-quality audio conferencing: fluency and awareness of attentional focus," Int'l Journal of Human-Computer Studies archive, vol. 49, no. 1, Jul. 1998.

[124] A. Vatakis and C. Spence, "Evaluating the influence of frame rate on the temporal aspects of audiovisual speech perception,” Neuroscience Letter, vol. 11, no. 405, Sep. 2006.

[125] W. Wu, Z. Yang, and K. Nahrstedt, "A study of visual context representation and control for remote sport learning tasks," in Proc. of World Conference on Educational Multimedia, Hypermedia and Telecommunications, 2008.

[126] ITU-G.107, “The E-model, a computational model for use in transmission planning,” 2008. [Online]. Available: http://www.itu.int/rec/T-REC-G.107/en/

[127] S. C. Y. Wang and A. Lou, "Subjective preference of spatio-temporal rate in video adaptation using multi-dimensional scalable coding," in Proc. of IEEE Int'l Conference on Multimedia and Expo, 2004.

[128] M. M. Yadavalli, G. and S. Hemami, "Frame rate preferences in low bit rate video," in Proc. of IEEE Int'l Conference on Image Processing, 2003.

[129] A. D. Bimbo, S.-F. Chang, and A. Smeulders, "Subjective evaluation of scalable video coding for content distribution," in Proceedings of the 18th International Conference on Multimedia, 2010. 
[130] W. Wu, A. Arefin, G. Kurillo, P. Agarwal, K. Nahrstedt, and R. Bajcsy, "Color-plus-depth level-ofdetail evaluation metric for $3 \mathrm{~d}$ teleimmersive video," in Proc. of ACM Int'l Conference on Multimedia, 2011.

[131] K.-T. Chen, C. C. Tu, and W.-C. Xiao, "Oneclick: A framework for measuring network quality of experience," in Proceedings of IEEE International Conference on Computer Communications, 2009.

[132] ITU-BT.1438, "Subjective assessment of stereoscopic television pictures," 2000.

[133] Z. Huang, B. Sat, and B. W. Wah, "Automated learning of play-out scheduling algorithms for improving the perceptual conversational quality in multi-party VoIP," in Proc. IEEE ICME, 2008.

[134] Y.-F. Ou, T. Liu, Z. Zhao, Z. Ma, and Y. Wang, "Modeling the impact of frame rate on perceptual quality of video," in Proc. of IEEE ICIP, 2008, pp. 689-692.

[135] K. Seshadrinathan, R. Soundararajan, A. Bovik, and L. K. Cormack, "Study of subjective and objective quality assessment of video," IEEE Transaction on Multimedia, vol. 19, no. 6, pp. 1427-1441, Jun. 2010.

[136] T. Oelbaum, H. Schwarz, M. Wien, and T. Wiegand, "Subjective performance evaluation of the SVC extension of H.264/AVC," in Proceedings of IEEE International Conference on Image Processing, 2008.

[137] M. Masry and S. S. Hemami, "An analysis of subjective quality in low bit rate video," in Proc. of IEEE International Conference on Image Processing, 2001.

[138] J. Kies, R. Williges, and M. Rosson, "Evaluating desktop video conferencing for distance learning," Elsevier Computers and Education, vol. 28, no. 2, pp. 79-91, Feb. 1997.

[139] G. Zhai, J. Cai, W. Lin, X. Yang, W. Zhang, and M. Etoh, "Cross-dimensional perceptual quality assessment for low bit-rate videos," IEEE Transaction on Multimedia, vol. 10, no. 7, pp. 1316-1324, Nov. 2008.

[140] Z. Lu, W. Lin, C. Boon, S. Kato, and et al, "Measuring the negative impact of frame dropping on perceptual visual quality," Human Vision and Electronic Imaging X, SPIE, vol. 5666, pp. 554-562, 2005 .

[141] R. Pastrana-Vidal, C. Jean, C. Colomes, and H. Cherifi, "Sporadic frame dropping impact on quality perception," Human Vision and Electronic Imaging IX, SPIE, vol. 5292, pp. 182-193, 2004.

[142] A. Vahedian, M. R. Frater, and J. F. Arnold, "Impact of audio on subjective assessment of video quality in videoconferencing applications," IEEE Transaction on Circuits System and Video Technology, vol. 11, no. 9, pp. 1059-1062, 2001.

[143] M. Jackson, A. H. Anderson, R. Mcewan, and J. Mullin, "Impact of video frame rate on communicative behaviour in two and four party groups," in Proc. of ACM Conference on Computer Supported Cooperative Work, 2000, pp. 11-20.

[144] H. Knoche and H. D. Meer, "Compensating for low frame rates," in CHI extended abstracts on Human factors in computing systems, 2005, pp. 1553-1556.

[145] S. Par and A. Kohlrausch, "Sensitivity to auditory-visual asynchrony and to jitter in auditory-visual timing," Human Vision and Electronic Imaging V, vol. 3959, pp. 234-242, Jun. 2000. 
[146] S. Winkler and C. Faller, "Perceived audiovisual quality of low-bitrate multimedia content," IEEE Transaction on Multimedia, vol. 8, no. 5, pp. 973-980, 2006.

[147] D. L. Richards, Telecommunication by Speech. London, UK: Butterworths, 1973.

[148] P. T. Brady, "Effects of transmission delay on conversational behaviour on echo-free telephone circuits," Bell System Technical Journal, vol. 50, no. 1, pp. 115-134, Jan. 1971.

[149] N. Kiatawaki and K. Itoh, "Pure delay effect on speech quality in telecommunications," IEEE Journal on Selected Areas of Communication, vol. 9, no. 4, pp. 586-593.

[150] ITU-G.114, "One-way transmission time," 2003. [Online]. Available: http://www.itu.int/rec/ T-REC-G.114/en/

[151] L. Mued, B. Lines, S. Furnell, and P. Reynolds, "The effects of lip synchronization in IP conferencing," in Proceedings of IEE International Conference on Visual Information Engineering, 2002, pp. 210-213.

[152] K.-T. Chen, C.-Y. Huang, P. Huang, and C.-L. Lei, "Quantifying Skype user satisfaction," in Proc. of ACM SIGCOMM, 2006.

[153] W. Wu, A. Arefin, R. Rivas, K. Nahrstedt, R. Sheppard, and Z. Yang, "Quality of experience in distributed interactive multimedia environments: Toward a theoretical framework," in Proceedings of ACM International Conference on Multimedia, 2009.

[154] W. Wu, A. Arefin, Z. Huang, P. Agarwal, and et al, "I'm the Jedi! - A case study of user experience in 3D tele-immersive gaming," in Proc. IEEE Int'l Symposium on Multimedia, 2010.

[155] J. You, L. Xing, A. Perkis, and X. Wang, "Perceptual quality assessment for stereoscopic images based on 2d image quality metrics and disparity analysis," in Proc. of International Workshop on Video Processing and Quality Metrics for Consumer Electronics, 2009.

[156] L. Xing, "A perceptual quality metric for stereoscopic crosstalk perception," in Proc. of IEEE International Conference on Image Processing, 2010.

[157] ITU-P.862, "Perceptual evaluation of speech quality (PESQ): An objective method for end-to-end speech quality assessment of narrow-band telephone networks and speech codecs," 2001. [Online]. Available: http://www.itu.int/rec/T-REC-P.862/en/

[158] B. Sat, Z. Huang, and B. W. Wah, "The design of a multi-party VoIP conferencing system over the Internet," in Proc. IEEE Int'l Symposium on Multimedia, Taichung, Taiwan, Dec. 2007, pp. 3-10.

[159] Z. Yang, B. Yu, K. Nahrstedt, and R. Bajscy, "A multi-stream adaptation framework for bandwidth management in 3D tele-immersion," in Proc. ACM Workshop on Network and Operating Systems Support for Digital Audio and Video, 2006.

[160] “NTP: the Network Timing Protocol,” 2009. [Online]. Available: http://www.ntp.org/

[161] P. Rangan, H. Vin, and S. Ramanathan, "Communication architectures and algorithms for media mixing in multimedia conferences," IEEE/ACM Transaction on Networking, vol. 1, no. 1, pp. 20-30, 1993. 
[162] P. Zarros, M. Lee, and T. Saadawi, "Interparticipant synchronization in real-time multimedia conferencing using feedback," IEEE/ACM Transaction on Networking, vol. 4, no. 2, pp. 173-180, 1996.

[163] B. Armstrong and S. Veettil, "Soft synchronization: synchronization for network-connected machine vision systems," IEEE Industrial Informatics, 2007.

[164] A. Borg, “Synchronization efficiency," Ph.D. thesis, New York University, 1981.

[165] “Google Hangout.” http://plus.google.com.

[166] R. Vasudevan, E. J. Lobaton, G. Kurillo, R. Bajcsy, and et al, "A methodology for remote virtual interaction in teleimmersive environments," in Proceedings of ACM Multimedia Systems Conference, 2010, pp. 281-292.

[167] OpenMP, "http://www.openmp.org/."

[168] W. Yuan and K. Nahrstedt, "Energy-efficient soft real-time CPU scheduling for mobile multimedia systems," in Proc. of ACM Symposium on Operating Systems Principles, Oct. 2003, pp. 149-163.

[169] S. Sadjadi and et al, "A modeling approach for estimating execution time of long-running scientific applications," in Proc. of IEEE Int'l Symposium on Parallel and Distributed Processing, Apr. 2008, pp. 1-8.

[170] A. Meddahi, H. Afifi, and G. Vanwormhoudt, “"MOSQoS”: Subjective VoIP Quality for Feedback Control and Dynamic QoS," Proc. of IEEE Int'l Conference on Communications, 2006.

[171] K. Deb, A. Pratap, S. Agarwal, and T. Meyarivan, "A fast and elitist multiobjective genetic algorithm: NSGA-II," IEEE Transactions on Evolutionary Computation, vol. 6, pp. 182-197, 2002. 